\title{
Asymmetric Synthesis of Boryl-Functionalized Cyclobutanols
}

\author{
Andrew Whyte,${ }^{\dagger}$ Bijan Mirabi,${ }^{\dagger}$ Alexa Torelli, ${ }^{\dagger}$ Liher Prieto, ${ }^{\dagger}{ }^{\dagger}$ Jonathan Bajohr,${ }^{\dagger}$ and Mark \\ Lautens $^{\dagger *}$ \\ <mark.lautens@utoronto.ca> \\ 'Davenport Research Laboratories, Department of Chemistry, University of Toronto, 80 St. George Street, Toronto, \\ Ontario M5S 3H6, Canada \\ ${ }^{7}$ Department of Organic Chemistry II, University of the Basque Country (UPV/EHU), 48080 Bilbao, Spain
}

\section{Table of Contents}

General Considerations.

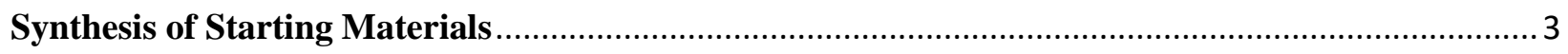

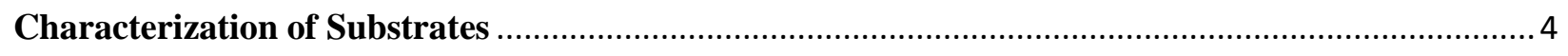

Procedure for catalytic reaction - General Procedure 3 ....................................................... 18

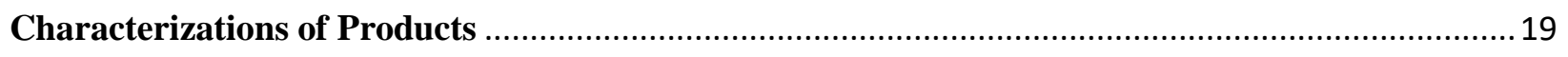

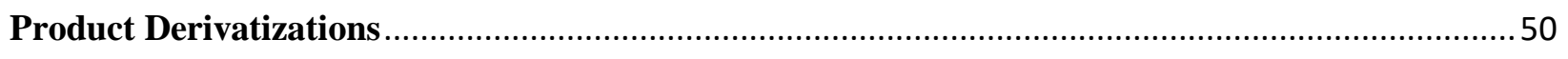

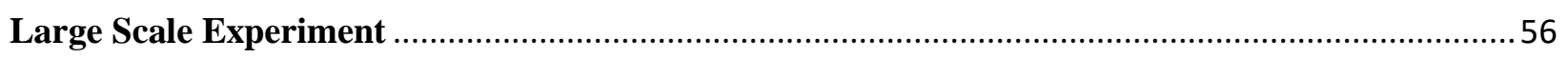

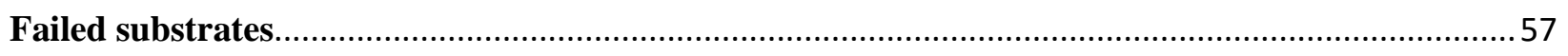

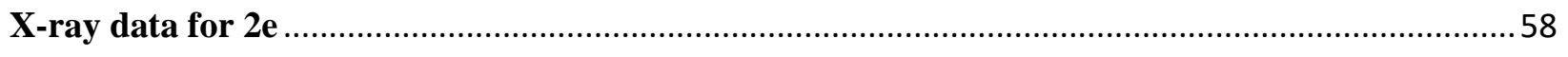

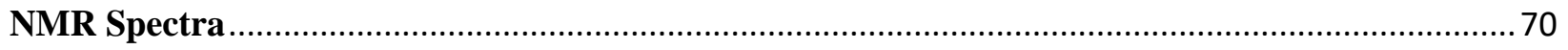




\section{General Considerations}

All reactions were performed under argon in a dry environment. Reaction progress was monitored by TLC using UV light or $\mathrm{KMnO}_{4}$ for visualization. THF was distilled over sodium/benzophenone. Anhydrous MTBE was purchased from Sigma Aldrich and used as received. Sodium tert-butoxide (97\%) was purchased from Sigma-Aldrich and was stored in a desiccator. Isopropanol was purchased from Caledon and used as received. Copper tetrakisacetonitrile hexafluorophosphate and $(S, S)$-BDPP were purchased from Strem and stored in a desiccator. 1,3,5-Trimethoxybenzene was purchased from Sigma Aldrich and used as received. Catalytic reactions were performed in $2 \mathrm{dr}$ vials, equipped with a Teflon septa (ThermoScientific National B7995-15) and a stir bar (Fisher cat no. 14-513-57, 12 x 4.5 mm). HPLC grade hexanes and isopropanol were purchased from Fisher and was used as received. Flash column chromatography was performed with Silicycle 46-60 $\mu \mathrm{m}$ silica gel. B2pin 2 was purchased from Combi-Blocks and dried under vacuum and stored in a desiccator. All other starting materials and reagents were purchased from Sigma Aldrich, Alfa Aesar, or CombiBlocks and were used as received.

${ }^{1} \mathrm{H},{ }^{13} \mathrm{C}$, and ${ }^{19} \mathrm{~F}$ NMR were obtained at $296 \mathrm{~K}$ on an Agilent DD2 500 equipped with a 5 mm Xses Cold Probe, or a Varian Mercury 300 or 400 or a Bruker Avance III 400.

Measurements were referenced to the solvent. NMR data is referenced as chemical shift, multiplicity, coupling constant, integration. Carbons directly bound to boron were not observed due to quadrupolar relaxation. NMR yields were obtained using 1,3,5-trimethoxybenzene as an internal standard. HRMS were obtained on a JEOL AccuTOF-DART or ESI performed at the Advanced Instrumentation for Molecular Structure (AIMS) at the University of Toronto. IR spectra were acquired on a Perkin-Elmer Spectrum 100 instrument with a single-bounce diamond/ZeSe ATR accessory. Data is presented in wavenumbers $\left(\mathrm{cm}^{-1}\right)$. Chiral HPLC was performed on Agilent 1100 or 1200 series operated by ChemStation LC 3D software. 
Synthesis of Starting Materials

Route 1<smiles>O=C(O)CCC(=O)c1[R]cccc1</smiles>

1) $\mathrm{HNMeOMe} \cdot \mathrm{HCl}$

EDC•HCl, DIPEA

2) $\mathrm{MePPh}_{3} \mathrm{Br}, \mathrm{KOtBu}$

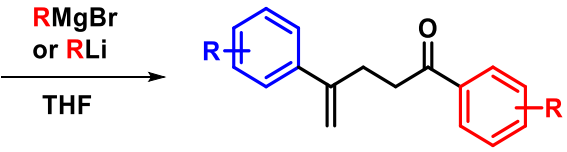

Route 2<smiles>[R][X]c1ccc(Br)cc1</smiles>

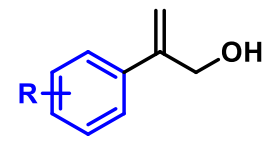

$$
\stackrel{\mathrm{CBr}_{4}, \mathrm{PPh}_{3}}{\longrightarrow}
$$

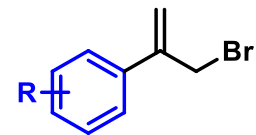<smiles>CCOC(=O)CC(=O)c1ccccc1</smiles>

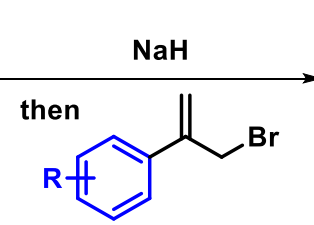<smiles>[R][R]1cccc(C(=C)CCC(=O)c2ccccc2)c1</smiles>

then $\mathrm{KOH}, \mathrm{MeOH}, \mathrm{H}_{2} \mathrm{O}$

Route 3<smiles>[R]C([R])C(=O)c1ccccc1</smiles><smiles>CCC(C)(C)C=C(CBr)c1ccccc1</smiles><smiles>C=C(CCC(=O)c1ccccc1)c1ccccc1</smiles>

Scheme S1. Synthesis of substrates

Route 1: following Org. Lett., 2018, 20, 1300-1303

Route 2: following J. Am. Chem. Soc. 2018, 140, 14647-14654

Route 3: following Org. Chem. Front. 2018, 5, 3476-3482

3 


\section{Characterization of Substrates}

5-phenylhex-5-en-2-one (1a)<smiles>C=C(CCC(C)=O)c1ccccc1</smiles>

Prepared following Org. Lett., 2018, 20, 1300-1303, consistent with literature spectra Chem. Comm., 2015, 51, 12771-12774

${ }^{1} \mathbf{H}$ NMR $\left(500 \mathrm{MHz}, \mathrm{CDCl}_{3}\right) \delta 7.43-7.36(\mathrm{~m}, 2 \mathrm{H}), 7.37-7.29(\mathrm{~m}, 2 \mathrm{H}), 7.32-7.24(\mathrm{~m}, 1 \mathrm{H})$, $5.29(\mathrm{dd}, J=1.2,0.6 \mathrm{~Hz}, 1 \mathrm{H}), 5.07(\mathrm{q}, J=1.2 \mathrm{~Hz}, 1 \mathrm{H}), 2.84-2.75(\mathrm{~m}, 2 \mathrm{H}), 2.63-2.54(\mathrm{~m}, 2 \mathrm{H})$, $2.12(\mathrm{~s}, 3 \mathrm{H})$.

${ }^{13}$ C NMR (126 MHz, $\left.\mathrm{CDCl}_{3}\right) \delta 208.1,147.1,140.6,128.4,127.6,126.1,112.7,42.4,30.0,29.2$.

\section{2-methyl-6-phenylhept-6-en-3-one (1b)}<smiles>C=C(CCC(=O)C(C)C)c1ccccc1</smiles>

Prepared following Org. Lett., 2018, 20, 1300-1303

Prepared on a $3 \mathrm{mmol}$ scale. Obtained a clear oil, $150 \mathrm{mg}$ (25\% yield).

${ }^{1} \mathbf{H}$ NMR $\left(500 \mathrm{MHz}, \mathrm{CDCl}_{3}\right) \delta 7.43-7.37(\mathrm{~m}, 2 \mathrm{H}), 7.37-7.29(\mathrm{~m}, 2 \mathrm{H}), 7.31-7.24(\mathrm{~m}, 1 \mathrm{H})$, $5.29(\mathrm{dd}, J=1.3,0.6 \mathrm{~Hz}, 1 \mathrm{H}), 5.08(\mathrm{q}, J=1.3 \mathrm{~Hz}, 1 \mathrm{H}), 2.83-2.76(\mathrm{~m}, 2 \mathrm{H}), 2.63-2.56(\mathrm{~m}, 2 \mathrm{H})$, $2.54(\mathrm{dq}, J=6.9 \mathrm{~Hz}, 1 \mathrm{H}), 1.06(\mathrm{~d}, J=6.9 \mathrm{~Hz}, 6 \mathrm{H})$.

${ }^{13}$ C NMR (126 MHz, $\left.\mathrm{CDCl}_{3}\right) \delta 213.9,147.4,140.6,128.4,127.5,126.1,112.7,40.9,40.9,39.1$, $29.3,18.2$.

IR (ATR) 2968, 1709, 1627, 1466, 1383, 1072, 894, 777, 702

HRMS (DART, M+H) Calculated for $\mathrm{C}_{14} \mathrm{H}_{19} \mathrm{O}_{1} 203.1436$, found 203.1433

\section{1-cyclohexyl-4-phenylpent-4-en-1-one (1c)}<smiles>C=C(CCC(=O)C1CCCCC1)c1ccccc1</smiles>

Prepared following Org. Lett., 2018, 20, 1300-1303 with organolithium, consistent with literature spectra Org. Lett., 2018, 20, 1300-1303 
${ }^{1} \mathbf{H}$ NMR $\left(399 \mathrm{MHz}, \mathrm{CDCl}_{3}\right) \delta 7.42-7.36(\mathrm{~m}, 2 \mathrm{H}), 7.36-7.30(\mathrm{~m}, 2 \mathrm{H}), 7.30-7.26(\mathrm{~m}, 1 \mathrm{H})$, $5.27(\mathrm{~d}, J=1.3 \mathrm{~Hz}, 1 \mathrm{H}), 5.06(\mathrm{q}, J=1.3 \mathrm{~Hz}, 1 \mathrm{H}), 2.82-2.72(\mathrm{~m}, 2 \mathrm{H}), 2.64-2.52(\mathrm{~m}, 2 \mathrm{H}), 2.29$ (tt, $J=11.3,3.3 \mathrm{~Hz}, 1 \mathrm{H}), 1.84-1.70(\mathrm{~m}, 4 \mathrm{H}), 1.69-1.61(\mathrm{~m}, 1 \mathrm{H}), 1.37-1.12(\mathrm{~m}, 5 \mathrm{H})$.

${ }^{13}$ C NMR $\left(100 \mathrm{MHz}, \mathrm{CDCl}_{3}\right) \delta 213.3,147.5,140.7,128.3,127.5,126.1,112.6,50.9,39.4,29.2$, 28.4, 25.8, 25.6.

\section{1-cyclopropyl-4-phenylpent-4-en-1-one (1d)}<smiles>C=C(CCC(=O)C1CC1)c1ccccc1</smiles>

Prepared following Org. Lett., 2018, 20, 1300-1303

Prepared on a $1.5 \mathrm{mmol}$ scale. Obtained a slightly yellow oil, $230 \mathrm{mg}$ (78\% yield).

${ }^{1} \mathbf{H}$ NMR $\left(500 \mathrm{MHz}, \mathrm{CDCl}_{3}\right) \delta 7.43-7.39(\mathrm{~m}, 2 \mathrm{H}), 7.36-7.31(\mathrm{~m}, 2 \mathrm{H}), 7.30-7.26(\mathrm{~m}, 1 \mathrm{H})$, $5.31-5.28(\mathrm{~m}, 1 \mathrm{H}), 5.09(\mathrm{q}, J=1.3 \mathrm{~Hz}, 1 \mathrm{H}), 2.86-2.79(\mathrm{~m}, 2 \mathrm{H}), 2.75-2.68(\mathrm{~m}, 2 \mathrm{H}), 1.88(\mathrm{tt}$, $J=7.8,4.6 \mathrm{~Hz}, 1 \mathrm{H}), 1.04-0.98(\mathrm{~m}, 2 \mathrm{H}), 0.86-0.82(\mathrm{~m}, 2 \mathrm{H})$.

${ }^{13}$ C NMR (126 MHz, $\left.\mathrm{CDCl}_{3}\right) \delta 210.1,147.3,140.7,128.4,127.5,126.1,112.7,42.2,29.4,20.5$, 20.5, 10.7 .

IR (ATR) 1696, 1627, 1494, 1444, 1385, 1195, 1083, 1014, 896, 776, 702

HRMS (DART, M+H) Calculated for $\mathrm{C}_{14} \mathrm{H}_{17} \mathrm{O}_{1} 201.1279$, found 201.1281

\section{1,4-diphenylpent-4-en-1-one (1e)}<smiles>C=C(CCC(=O)c1ccccc1)c1ccccc1</smiles>

Prepared following Org. Lett., 2018, 20, 1300-1303, consistent with literature spectra Org. Lett., 2018, 20, 1300-1303

${ }^{1} \mathbf{H}$ NMR $\left(500 \mathrm{MHz}, \mathrm{CDCl}_{3}\right) \delta 7.96-7.90(\mathrm{~m}, 2 \mathrm{H}), 7.57-7.52(\mathrm{~m}, 1 \mathrm{H}), 7.47-7.41(\mathrm{~m}, 4 \mathrm{H})$, $7.38-7.33(\mathrm{~m}, 2 \mathrm{H}), 7.32-7.27(\mathrm{~m}, 1 \mathrm{H}), 5.35(\mathrm{~d}, J=1.1 \mathrm{~Hz}, 1 \mathrm{H}), 5.16(\mathrm{q}, J=1.1 \mathrm{~Hz}, 1 \mathrm{H}), 3.17$ $-3.10(\mathrm{~m}, 2 \mathrm{H}), 3.02-2.94(\mathrm{~m}, 2 \mathrm{H})$.

${ }^{13} \mathbf{C}$ NMR $\left(126 \mathrm{MHz}, \mathrm{CDCl}_{3}\right) \delta 199.5,147.3,140.6,136.9,133.0,128.6,128.4,128.0,127.6$, 126.1, 112.9, 37.5, 29.7.

\section{4-phenyl-1-(p-tolyl)pent-4-en-1-one (1f)}


<smiles>C=C(CCC(=O)c1ccc(C)cc1)c1ccccc1</smiles>

Prepared following Org. Lett., 2018, 20, 1300-1303, consistent with literature spectra Org. Lett., 2018, 20, 1300-1303

${ }^{1} \mathbf{H}$ NMR $\left(500 \mathrm{MHz}, \mathrm{CDCl}_{3}\right) \delta 7.83(\mathrm{~d}, J=8.3 \mathrm{~Hz}, 2 \mathrm{H}), 7.47-7.43(\mathrm{~m}, 2 \mathrm{H}), 7.38-7.32(\mathrm{~m}$, 2H), $7.31-7.27(\mathrm{~m}, 1 \mathrm{H}), 7.24(\mathrm{~d}, J=8.3 \mathrm{~Hz}, 2 \mathrm{H}), 5.34(\mathrm{~d}, J=1.1 \mathrm{~Hz}, 1 \mathrm{H}), 5.15(\mathrm{q}, J=1.1 \mathrm{~Hz}$, $1 \mathrm{H}), 3.15-3.07(\mathrm{~m}, 2 \mathrm{H}), 3.03-2.93(\mathrm{~m}, 2 \mathrm{H}), 2.40(\mathrm{~s}, 3 \mathrm{H})$.

${ }^{13}$ C NMR $\left(126 \mathrm{MHz}, \mathrm{CDCl}_{3}\right) \delta$ 199.1, 147.4, 143.8, 140.6, 134.4, 129.2, 128.4, 128.1, 127.6, 126.1, 112.8, 37.4, 29.8, 21.6, 21.6.

1-(4-methoxyphenyl)-4-phenylpent-4-en-1-one (1g)<smiles>C=C(CCC(=O)c1ccc(OC)cc1)c1ccccc1</smiles>

Prepared following Org. Lett., 2018, 20, 1300-1303, consistent with literature spectra Org. Lett., 2018, 20, 1300-1303

${ }^{1} \mathbf{H}$ NMR $\left(500 \mathrm{MHz}, \mathrm{CDCl}_{3}\right) \delta 7.91(\mathrm{~d}, J=8.9 \mathrm{~Hz}, 2 \mathrm{H}), 7.46-7.43(\mathrm{~m}, 2 \mathrm{H}), 7.37-7.31(\mathrm{~m}$, $2 \mathrm{H}), 7.31-7.27(\mathrm{~m}, 1 \mathrm{H}), 6.91(\mathrm{~d}, J=8.9 \mathrm{~Hz}, 2 \mathrm{H}), 5.33(\mathrm{~d}, J=1.2 \mathrm{~Hz}, 1 \mathrm{H}), 5.14(\mathrm{q}, J=1.2 \mathrm{~Hz}$, $1 \mathrm{H}), 3.85(\mathrm{~s}, 3 \mathrm{H}), 3.11-3.04(\mathrm{~m}, 2 \mathrm{H}), 3.00-2.90(\mathrm{~m}, 2 \mathrm{H})$.

${ }^{13}$ C NMR (126 MHz, $\left.\mathrm{CDCl}_{3}\right) \delta 198.1,163.4,147.5,140.6,130.3,130.0,128.4,127.6,126.1$, $113.7,112.8,55.4,37.1,29.9$.

\section{1-(4-fluorophenyl)-4-phenylpent-4-en-1-one (1h)}<smiles>C=C(CCC(=O)c1ccc(F)cc1)c1ccccc1</smiles>

Prepared following Org. Lett., 2018, 20, 1300-1303

Prepared on a $5 \mathrm{mmol}$ scale from the Weinreb amide. Obtained an off-white solid, $1.00 \mathrm{~g} \mathrm{(79 \%}$ yield).

${ }^{1} \mathbf{H}$ NMR $\left(500 \mathrm{MHz}, \mathrm{CDCl}_{3}\right) \delta 8.00-7.87(\mathrm{~m}, 2 \mathrm{H}), 7.46-7.42(\mathrm{~m}, 2 \mathrm{H}), 7.37-7.32(\mathrm{~m}, 2 \mathrm{H})$, $7.31-7.27(\mathrm{~m}, 1 \mathrm{H}), 7.12-7.07(\mathrm{~m}, 2 \mathrm{H}), 5.34(\mathrm{~d}, J=1.1 \mathrm{~Hz}, 1 \mathrm{H}), 5.14(\mathrm{q}, J=1.1 \mathrm{~Hz}, 1 \mathrm{H}), 3.13$ $-3.06(\mathrm{~m}, 2 \mathrm{H}), 3.00-2.94(\mathrm{~m}, 2 \mathrm{H})$.

${ }^{13} \mathrm{C}$ NMR $\left(126 \mathrm{MHz}, \mathrm{CDCl}_{3}\right) \delta 197.9,165.7(\mathrm{~d}, J=254.6 \mathrm{~Hz}), 147.2,140.5,133.3(\mathrm{~d}, J=3.0$ $\mathrm{Hz}), 130.6(\mathrm{~d}, J=9.3 \mathrm{~Hz}), 128.5,127.7,126.1,115.6$ (d, $J=21.8 \mathrm{~Hz}), 113.0,37.4,29.7$. 
${ }^{19}$ F NMR $\left(377 \mathrm{MHz}, \mathrm{CDCl}_{3}\right) \delta-105.3$.

IR (ATR) 1628, 1592, 1443, 1408, 1309, 1258, 905, 824, 719, 700, 600

HRMS (DART, $\mathrm{M}+\mathrm{H}$ ) Calculated for $\mathrm{C}_{17} \mathrm{H}_{16} \mathrm{~F}_{1} \mathrm{O}_{1} 255.1185$, found 255.1179

MP $62{ }^{\circ} \mathrm{C}$

4-phenyl-1-(4-(trifluoromethyl)phenyl)pent-4-en-1-one (1i)<smiles>C=C(CCC(=O)c1ccc(C(F)(F)F)cc1)c1ccccc1</smiles>

Prepared following Org. Lett., 2018, 20, 1300-1303, consistent with literature spectra Org. Lett., 2018, 20, 1300-1303

${ }^{1} \mathbf{H}$ NMR $\left(500 \mathrm{MHz}, \mathrm{CDCl}_{3}\right) \delta 8.01(\mathrm{~d}, J=8.1 \mathrm{~Hz}, 2 \mathrm{H}), 7.70(\mathrm{~d}, J=8.1 \mathrm{~Hz}, 2 \mathrm{H}), 7.45-7.40(\mathrm{~m}$, $2 \mathrm{H}), 7.38-7.32(\mathrm{~m}, 2 \mathrm{H}), 7.32-7.27(\mathrm{~m}, 1 \mathrm{H}), 5.35(\mathrm{~d}, J=1.1 \mathrm{~Hz}, 1 \mathrm{H}), 5.15(\mathrm{~d}, J=1.1 \mathrm{~Hz}, 1 \mathrm{H})$, $3.17-3.12(\mathrm{~m}, 2 \mathrm{H}), 3.02-2.96(\mathrm{~m}, 2 \mathrm{H})$.

${ }^{13} \mathrm{C}$ NMR $\left(126 \mathrm{MHz}, \mathrm{CDCl}_{3}\right) \delta 198.5,147.0,140.4,139.5(\mathrm{q}, J=1.2 \mathrm{~Hz}), 134.3(\mathrm{q}, J=32.7$ Hz), 128.5, 128.3, 127.7, 126.1, 125.6 (q, $J=3.7 \mathrm{~Hz}), 123.6$ (q, $J=272.7 \mathrm{~Hz}), 113.1,37.8,29.6$.

${ }^{19}$ F NMR $\left(377 \mathrm{MHz}, \mathrm{CDCl}_{3}\right) \delta-63.1$.

\section{4-phenyl-1-(m-tolyl)pent-4-en-1-one (1j)}<smiles>C=C(CCC(=O)c1cccc(C)c1)c1ccccc1</smiles>

Prepared following Org. Lett., 2018, 20, 1300-1303, consistent with literature spectra Org. Lett., 2018, 20, 1300-1303

${ }^{1} \mathbf{H}$ NMR $\left(500 \mathrm{MHz}, \mathrm{CDCl}_{3}\right) \delta 7.76-7.70(\mathrm{~m}, 2 \mathrm{H}), 7.49-7.42(\mathrm{~m}, 2 \mathrm{H}), 7.38-7.28(\mathrm{~m}, 5 \mathrm{H})$, $5.35(\mathrm{~d}, J=1.2 \mathrm{~Hz}, 1 \mathrm{H}), 5.16(\mathrm{q}, J=1.3 \mathrm{~Hz}, 1 \mathrm{H}), 3.16-3.10(\mathrm{~m}, 2 \mathrm{H}), 3.02-2.95(\mathrm{~m}, 2 \mathrm{H}), 2.40$ (s, 3H).

${ }^{13} \mathrm{C}$ NMR $\left(126 \mathrm{MHz}, \mathrm{CDCl}_{3}\right) \delta 199.7,147.4,140.6,138.3,136.9,133.8,128.6,128.6,128.4$, 127.6, 126.1, 125.3, 112.9, 37.5, 29.8, 21.3.

1-(3-methoxyphenyl)-4-phenylpent-4-en-1-one (1k)<smiles>C=C(CCC(=O)c1cccc(OC)c1)c1ccccc1</smiles> 
Prepared following Org. Lett., 2018, 20, 1300-1303

Prepared on a $4 \mathrm{mmol}$ scale from the Weinreb amide. Obtained a white solid, $452 \mathrm{mg}$ (42\% yield).

${ }^{1} \mathbf{H}$ NMR $\left(500 \mathrm{MHz}, \mathrm{CDCl}_{3}\right) \delta 7.51-7.43(\mathrm{~m}, 4 \mathrm{H}), 7.38-7.31(\mathrm{~m}, 3 \mathrm{H}), 7.31-7.27(\mathrm{~m}, 1 \mathrm{H})$, 7.09 (ddd, $J=8.3,2.7,1.0 \mathrm{~Hz}, 1 \mathrm{H}), 5.34(\mathrm{~d}, J=1.3 \mathrm{~Hz}, 1 \mathrm{H}), 5.15(\mathrm{q}, J=1.3 \mathrm{~Hz}, 1 \mathrm{H}), 3.84$ (s, $3 \mathrm{H}), 3.15-3.08(\mathrm{~m}, 2 \mathrm{H}), 3.00-2.94(\mathrm{~m}, 2 \mathrm{H})$.

${ }^{13}$ C NMR $\left(126 \mathrm{MHz}, \mathrm{CDCl}_{3}\right) \delta 199.3,159.8,147.3,140.6,138.3,129.5,128.4,127.6,126.1$, 120.7, 119.5, 112.9, 112.3, 55.4, 37.6, 29.8 .

IR (ATR) 1678, 1591, 1465, 1427, 1337, 1163, 1026, 1261, 1012, 891, 774, 683, 584

HRMS (DART, M+H) Calculated for $\mathrm{C}_{18} \mathrm{H}_{19} \mathrm{O}_{2} 267.1385$, found 267.1380

MP $32{ }^{\circ} \mathrm{C}$

\section{1-(3-fluorophenyl)-4-phenylpent-4-en-1-one (11)}<smiles>C=C(CCC(=O)c1cccc(F)c1)c1ccccc1</smiles>

Prepared following Org. Lett., 2018, 20, 1300-1303, consistent with literature spectra Org. Lett., 2018, 20, 1300-1303

${ }^{1} \mathbf{H}$ NMR $\left(500 \mathrm{MHz}, \mathrm{CDCl}_{3}\right) \delta 7.69$ (ddd, $J=7.7,1.6,1.0 \mathrm{~Hz}, 1 \mathrm{H}$ ), 7.61 (ddd, $J=9.5,2.7,1.6$ $\mathrm{Hz}, 1 \mathrm{H}), 7.47-7.42(\mathrm{~m}, 2 \mathrm{H}), 7.42-7.38(\mathrm{~m}, 1 \mathrm{H}), 7.38-7.33(\mathrm{~m}, 2 \mathrm{H}), 7.32-7.28(\mathrm{~m}, 1 \mathrm{H})$, $7.24(\mathrm{tdd}, J=8.3,2.5,1.0 \mathrm{~Hz}, 1 \mathrm{H}), 5.35(\mathrm{~d}, J=1.1 \mathrm{~Hz}, 1 \mathrm{H}), 5.15(\mathrm{q}, J=1.1 \mathrm{~Hz}, 1 \mathrm{H}), 3.14-3.08$ (m, 2H), $3.01-2.93(\mathrm{~m}, 2 \mathrm{H})$.

${ }^{13}$ C NMR $\left(126 \mathrm{MHz}, \mathrm{CDCl}_{3}\right) \delta 198.2(\mathrm{~d}, J=2.0 \mathrm{~Hz}), 162.8(\mathrm{~d}, J=247.8 \mathrm{~Hz}), 147.1,140.5$, $139.0(\mathrm{~d}, J=6.0 \mathrm{~Hz}), 130.2(\mathrm{~d}, J=7.6 \mathrm{~Hz}), 128.5,127.7,126.1,123.8(\mathrm{~d}, J=3.0 \mathrm{~Hz}), 120.0(\mathrm{~d}$, $J=21.5 \mathrm{~Hz}), 114.8(\mathrm{~d}, J=22.2 \mathrm{~Hz}), 113.0,37.6,29.6$.

${ }^{19} \mathbf{F}$ NMR $\left(377 \mathrm{MHz}, \mathrm{CDCl}_{3}\right) \delta-111.8$.

\section{4-phenyl-1-(3-(trifluoromethyl)phenyl)pent-4-en-1-one (1m)}<smiles>C=C(CCC(=O)c1cccc(C(F)(F)F)c1)c1ccccc1</smiles>

Prepared following Org. Lett., 2018, 20, 1300-1303

Prepared on a $6 \mathrm{mmol}$ scale from the Weinreb amide. Obtained a clear oil, $565 \mathrm{mg}$ ( $31 \%$ yield). 
${ }^{1} \mathbf{H}$ NMR $\left(500 \mathrm{MHz}, \mathrm{CDCl}_{3}\right) \delta 8.16(\mathrm{~s}, 1 \mathrm{H}), 8.09(\mathrm{~d}, J=7.8 \mathrm{~Hz}, 1 \mathrm{H}), 7.83-7.78(\mathrm{~m}, 1 \mathrm{H}), 7.58$ (tt, $J=7.8,0.6 \mathrm{~Hz}, 1 \mathrm{H}), 7.47-7.39(\mathrm{~m}, 2 \mathrm{H}), 7.39-7.32(\mathrm{~m}, 2 \mathrm{H}), 7.32-7.28(\mathrm{~m}, 1 \mathrm{H}), 5.35(\mathrm{~d}, J$ $=1.1 \mathrm{~Hz}, 1 \mathrm{H}), 5.16(\mathrm{q}, J=1.3 \mathrm{~Hz}, 1 \mathrm{H}), 3.18-3.12(\mathrm{~m}, 2 \mathrm{H}), 3.02-2.92(\mathrm{~m}, 2 \mathrm{H})$.

${ }^{13}$ C NMR (126 MHz, $\left.\mathrm{CDCl}_{3}\right) \delta 198.1,147.0,140.3,137.4,131.2(\mathrm{q}, J=32.8 \mathrm{~Hz}), 131.2,129.4$ $(\mathrm{q}, J=3.6 \mathrm{~Hz}), 129.2(\mathrm{~d}, J=0.9 \mathrm{~Hz}), 128.5,127.7,126.1,124.8(\mathrm{q}, J=3.8 \mathrm{~Hz}), 123.7(\mathrm{q}, J=$ $272.6 \mathrm{~Hz}), 113.2,37.6,29.6$.

${ }^{19} \mathbf{F}$ NMR $\left(377 \mathrm{MHz}, \mathrm{CDCl}_{3}\right) \delta-62.8$.

IR (ATR) 1692, 1612, 1437, 1328, 1270, 1166, 1123, 1071, 898, 778, 693

HRMS (DART, $\mathrm{M}+\mathrm{H}$ ) Calculated for $\mathrm{C}_{18} \mathrm{H}_{16} \mathrm{~F}_{3} \mathrm{O}_{1} 305.1153$, found 305.1159

ethyl 3-(4-phenylpent-4-enoyl)benzoate (1n)<smiles>C=C(CCC(=O)c1cccc(C(=O)OCC)c1)c1ccccc1</smiles>

Prepared following Org. Lett., 2018, 20, 1300-1303.

Prepared on a $1.5 \mathrm{mmol}$ scale from the Weinreb amide. Obtained a clear oil, $296 \mathrm{mg}$ (64\% yield).

${ }^{1} \mathbf{H}$ NMR $\left(500 \mathrm{MHz}, \mathrm{CDCl}_{3}\right) \delta 8.55(\mathrm{td}, J=1.8,0.5 \mathrm{~Hz}, 1 \mathrm{H}), 8.21(\mathrm{dt}, J=7.8,1.8 \mathrm{~Hz}, 1 \mathrm{H}), 8.10$ (ddd, $J=7.8,1.8,1.2 \mathrm{~Hz}, 1 \mathrm{H}), 7.52(\mathrm{td}, J=7.8,0.5 \mathrm{~Hz}, 1 \mathrm{H}), 7.46-7.42(\mathrm{~m}, 2 \mathrm{H}), 7.36-7.32$ (m, 2H), $7.31-7.26(\mathrm{~m}, 1 \mathrm{H}), 5.34(\mathrm{~d}, J=1.1 \mathrm{~Hz}, 1 \mathrm{H}), 5.15(\mathrm{q}, J=1.3 \mathrm{~Hz}, 1 \mathrm{H}), 4.40(\mathrm{q}, J=7.2$ $\mathrm{Hz}, 2 \mathrm{H}), 3.21-3.13(\mathrm{~m}, 2 \mathrm{H}), 3.03-2.93(\mathrm{~m}, 2 \mathrm{H}), 1.41(\mathrm{t}, J=7.1 \mathrm{~Hz}, 3 \mathrm{H})$.

${ }^{13}$ C NMR $\left(126 \mathrm{MHz}, \mathrm{CDCl}_{3}\right) \delta 198.7,165.8,147.1,140.5,137.1,133.8,132.0,131.0,129.1$, $128.8,128.5,127.7,126.1,113.0,61.4,37.6,29.6,14.3$.

IR (ATR) 2979, 1724, 1693, 1602, 1443, 1366, 1284, 1192, 1094, 1024, 899, 780, 697, 749

HRMS (DART, $\mathrm{M}+\mathrm{H}$ ) Calculated for $\mathrm{C}_{20} \mathrm{H}_{21} \mathrm{O}_{3} 309.14852$, found 309.14870

\section{3-(4-phenylpent-4-enoyl)benzonitrile (10)}<smiles>C=C(CCC(=O)c1cccc(C#N)c1)c1ccccc1</smiles>

Prepared following Org. Lett., 2018, 20, 1300-1303.

Prepared on a $1.5 \mathrm{mmol}$ scale from the Weinreb amide. Obtained a white solid, $222 \mathrm{mg}$ (64\% yield). 
${ }^{1} \mathbf{H}$ NMR $\left(500 \mathrm{MHz}, \mathrm{CDCl}_{3}\right) \delta 8.16(\mathrm{t}, J=1.5 \mathrm{~Hz}, 1 \mathrm{H}), 8.11(\mathrm{dt}, J=7.9,1.5 \mathrm{~Hz}, 1 \mathrm{H}), 7.81(\mathrm{dt}, J$ $=7.9,1.5 \mathrm{~Hz}, 1 \mathrm{H}), 7.57(\mathrm{t}, J=7.9 \mathrm{~Hz}, 1 \mathrm{H}), 7.45-7.39(\mathrm{~m}, 2 \mathrm{H}), 7.38-7.31(\mathrm{~m}, 2 \mathrm{H}), 7.33-7.26$ $(\mathrm{m}, 1 \mathrm{H}), 5.33(\mathrm{~d}, J=1.1 \mathrm{~Hz}, 1 \mathrm{H}), 5.14(\mathrm{~d}, J=1.3 \mathrm{~Hz}, 1 \mathrm{H}), 3.11(\mathrm{~m}, 2 \mathrm{H}), 2.98(\mathrm{~m}, 2 \mathrm{H})$.

${ }^{13}$ C NMR $\left(126 \mathrm{MHz}, \mathrm{CDCl}_{3}\right) \delta 197.4,146.8,140.3,137.5,135.9,131.9,131.7,129.6,128.5$, $127.8,126.1,117.9,113.3,113.1,37.5,29.5$.

IR (ATR) 2906, 2231, 1693, 1611, 1495, 1424, 1321, 1256, 1215, 1143, 1024, 902, 780, 709, 674

HRMS (DART, M+H) Calculated for $\mathrm{C}_{18} \mathrm{H}_{16} \mathrm{NO}, 262.12264$ found 262.12245

MP $37{ }^{\circ} \mathrm{C}$

\section{4-phenyl-1-(o-tolyl)pent-4-en-1-one (1p)}<smiles>C=C(CCC(=O)c1ccccc1C)c1ccccc1</smiles>

Prepared following $\mathrm{Org}$. Lett., 2018, 20, 1300-1303, consistent with literature spectra $\mathrm{Org}$. Lett., 2018, 20, 1300-1303

${ }^{1} \mathbf{H}$ NMR $\left(500 \mathrm{MHz}, \mathrm{CDCl}_{3}\right) \delta 7.56(\mathrm{dd}, J=7.7,1.4 \mathrm{~Hz}, 1 \mathrm{H}), 7.46-7.42(\mathrm{~m}, 2 \mathrm{H}), 7.38-7.32$ $(\mathrm{m}, 3 \mathrm{H}), 7.32-7.27(\mathrm{~m}, 1 \mathrm{H}), 7.26-7.20(\mathrm{~m}, 2 \mathrm{H}), 5.34(\mathrm{~d}, J=1.1 \mathrm{~Hz}, 1 \mathrm{H}), 5.14(\mathrm{q}, J=1.3 \mathrm{~Hz}$, $1 \mathrm{H}), 3.09-3.03(\mathrm{~m}, 2 \mathrm{H}), 2.99-2.93(\mathrm{~m}, 2 \mathrm{H}), 2.51(\mathrm{~s}, 3 \mathrm{H})$.

${ }^{13}$ C NMR $\left(126 \mathrm{MHz}, \mathrm{CDCl}_{3}\right) \delta 203.6,147.3,140.7,138.0,138.0,131.9,131.2,128.4,128.4$, 127.6, 126.1, 125.6, 112.8, 40.3, 29.9, 21.3.

\section{4-phenyl-1-(thiophen-2-yl)pent-4-en-1-one (1q)}<smiles>C=C(CCC(=O)c1cccs1)c1ccccc1</smiles>

Prepared following Org. Lett., 2018, 20, 1300-1303, consistent with literature spectra Org. Lett., 2018, 20, 1300-1303.

${ }^{1} \mathbf{H}$ NMR $\left(500 \mathrm{MHz}, \mathrm{CDCl}_{3}\right) \delta 7.63(\mathrm{dd}, J=3.8,1.2 \mathrm{~Hz}, 1 \mathrm{H}), 7.61(\mathrm{dd}, J=4.9,1.1 \mathrm{~Hz}, 1 \mathrm{H}), 7.46$ $-7.41(\mathrm{~m}, 2 \mathrm{H}), 7.37-7.31(\mathrm{~m}, 2 \mathrm{H}), 7.31-7.27(\mathrm{~m}, 1 \mathrm{H}), 7.09(\mathrm{dd}, J=5.0,3.8 \mathrm{~Hz}, 1 \mathrm{H}), 5.34(\mathrm{~d}$, $J=1.2 \mathrm{~Hz}, 1 \mathrm{H}), 5.15$ (q, $J=1.3 \mathrm{~Hz}, 1 \mathrm{H}), 3.09-3.03(\mathrm{~m}, 2 \mathrm{H}), 3.00-2.94(\mathrm{~m}, 2 \mathrm{H})$.

${ }^{13}$ C NMR $\left(126 \mathrm{MHz}, \mathrm{CDCl}_{3}\right) \delta 192.4,147.1,144.2,140.4,133.6,131.8,128.4,128.0,127.6$, $126.1,113.0,38.2,30.0$.

\section{1-(1-methyl-1H-indol-5-yl)-4-phenylpent-4-en-1-one (1r)}


<smiles>C=C(CCC(=O)c1ccc2c(ccn2C)c1)c1ccccc1</smiles>

Prepared following Org. Lett., 2018, 20, 1300-1303

Prepared on $3 \mathrm{mmol}$ scale using organolithium. Obtained $414 \mathrm{mg}$ of a white solid (48\% yield). Product is not stable and slowly turns to a purple solid at room temperature.

${ }^{1} \mathbf{H}$ NMR $\left(500 \mathrm{MHz}, \mathrm{CDCl}_{3}\right) \delta 8.27(\mathrm{dd}, J=1.7,0.7 \mathrm{~Hz}, 1 \mathrm{H}), 7.90(\mathrm{dd}, J=8.7,1.7 \mathrm{~Hz}, 1 \mathrm{H}), 7.51$ $-7.47(\mathrm{~m}, 2 \mathrm{H}), 7.39-7.28(\mathrm{~m}, 4 \mathrm{H}), 7.10(\mathrm{~d}, J=3.2 \mathrm{~Hz}, 1 \mathrm{H}), 6.58(\mathrm{dd}, J=3.2,0.9 \mathrm{~Hz}, 1 \mathrm{H}), 5.36$ $(\mathrm{d}, J=1.3 \mathrm{~Hz}, 1 \mathrm{H}), 5.18(\mathrm{q}, J=1.3 \mathrm{~Hz}, 1 \mathrm{H}), 3.80(\mathrm{~s}, 3 \mathrm{H}), 3.25-3.19(\mathrm{~m}, 2 \mathrm{H}), 3.05-2.99(\mathrm{~m}$, $2 \mathrm{H})$.

${ }^{13}$ C NMR $\left(126 \mathrm{MHz}, \mathrm{CDCl}_{3}\right) \delta 199.6,147.7,140.8,139.1,130.4,129.1,128.4,127.9,127.5$, $126.2,122.8,121.7,112.7,109.1,103.0,37.4,33.0,30.2$.

IR (ATR) 1670, 1613, 1495, 1345, 1317, 1256, 1153, 1044, 988, 871, 781, 739, 696

HRMS (DART, M+H) Calculated for $\mathrm{C}_{20} \mathrm{H}_{20} \mathrm{~N}_{1} \mathrm{O}_{1} 290.1545$, found 290.1547

MP $58{ }^{\circ} \mathrm{C}$

\section{1-phenyl-4-(p-tolyl)pent-4-en-1-one (1s)}<smiles>C=C(CCC(=O)c1ccccc1)c1ccc(C)cc1</smiles>

Prepared following Org. Lett., 2018, 20, 1300-1303, consistent with literature spectra Org. Lett., 2018, 20, 1300-1303

${ }^{1} \mathbf{H}$ NMR $\left(500 \mathrm{MHz}, \mathrm{CDCl}_{3}\right) \delta 7.97-7.91(\mathrm{~m}, 2 \mathrm{H}), 7.60-7.52(\mathrm{~m}, 1 \mathrm{H}) .7 .48-7.41(\mathrm{~m}, 2 \mathrm{H})$, $7.40-7.33(\mathrm{~m}, 2 \mathrm{H}), 7.20-7.14(\mathrm{~m}, 2 \mathrm{H}), 5.33(\mathrm{~d}, J=1.3 \mathrm{~Hz}, 1 \mathrm{H}), 5.12(\mathrm{q}, J=1.3 \mathrm{~Hz}, 1 \mathrm{H}), 3.19$ $-3.11(\mathrm{~m}, 2 \mathrm{H}), 3.02-2.94(\mathrm{~m}, 2 \mathrm{H}), 2.37(\mathrm{~s}, 3 \mathrm{H})$.

${ }^{13} \mathrm{C}$ NMR $\left(126 \mathrm{MHz}, \mathrm{CDCl}_{3}\right) \delta 199.6,147.1,137.6,137.4,136.9,133.0,129.1,128.6,128.0$, 126.0, 112.1, 37.6, 29.7, 21.1.

\section{1-(4-fluorophenyl)-4-(p-tolyl)pent-4-en-1-one (1t)}<smiles>C=C(CCC(=O)c1ccc(F)cc1)c1ccc(C)cc1</smiles>

Prepared following Org. Lett., 2018, 20, 1300-1303. 
Prepared on a $6 \mathrm{mmol}$ scale from the Weinreb amide. Obtained a white solid, $557 \mathrm{mg}$ (35\% yield).

${ }^{1} \mathbf{H}$ NMR $\left(500 \mathrm{MHz}, \mathrm{CDCl}_{3}\right) \delta 8.00-7.88(\mathrm{~m}, 2 \mathrm{H}), 7.34(\mathrm{~d}, J=8.1 \mathrm{~Hz}, 2 \mathrm{H}), 7.16(\mathrm{~d}, J=8.1 \mathrm{~Hz}$, $2 \mathrm{H}), 7.13-7.07(\mathrm{~m}, 2 \mathrm{H}), 5.31(\mathrm{~d}, J=1.3 \mathrm{~Hz}, 1 \mathrm{H}), 5.10(\mathrm{q}, J=1.3 \mathrm{~Hz}, 1 \mathrm{H}), 3.12-3.07(\mathrm{~m}, 2 \mathrm{H})$, $2.98-2.92(\mathrm{~m}, 2 \mathrm{H}), 2.36(\mathrm{~s}, 3 \mathrm{H})$.

${ }^{13}$ C NMR (126 MHz, $\left.\mathrm{CDCl}_{3}\right) \delta 197.9,165.7(\mathrm{~d}, J=254.6 \mathrm{~Hz}), 147.0,137.5(\mathrm{~d}, J=10.2 \mathrm{~Hz})$, $133.3(\mathrm{~d}, J=3.1 \mathrm{~Hz}), 130.6(\mathrm{~d}, J=9.3 \mathrm{~Hz}), 129.1,126.0,115.6(\mathrm{~d}, J=21.8 \mathrm{~Hz}), 112.2,37.5$, 29.7, 21.1 .

${ }^{19}$ F NMR (377 MHz, ) $\delta-105.5$.

IR (ATR) 1678, 1594, 1504, 1406, 1324, 1227, 1199, 1156, 988, 901, 829, 790, 739, 593

HRMS (DART, M+H) Calculated for $\mathrm{C}_{18} \mathrm{H}_{18} \mathrm{~F}_{1} \mathrm{O}_{1} 269.1342$, found 269.1350

MP $83{ }^{\circ} \mathrm{C}$

\section{4-(4-fluorophenyl)-1-phenylpent-4-en-1-one (1u)}<smiles>C=C(CCC(=O)c1ccccc1)c1ccc(F)cc1</smiles>

Prepared following Org. Lett., 2018, 20, 1300-1303.

Prepared on $5.5 \mathrm{mmol}$ scale from the Weinreb amide. Obtained a slightly yellow solid, $612 \mathrm{mg}$ (44\% yield).

${ }^{1} \mathbf{H}$ NMR $\left(500 \mathrm{MHz}, \mathrm{CDCl}_{3}\right) \delta 7.92(\mathrm{dd}, J=8.4,1.3 \mathrm{~Hz}, 2 \mathrm{H}), 7.58-7.52(\mathrm{~m}, 1 \mathrm{H}), 7.44(\mathrm{ddt}, J=$ 8.4, 6.7, $0.9 \mathrm{~Hz}, 2 \mathrm{H}), 7.42-7.38(\mathrm{~m}, 2 \mathrm{H}), 7.02(\mathrm{t}, J=8.7 \mathrm{~Hz}, 2 \mathrm{H}), 5.28(\mathrm{~d}, J=1.3 \mathrm{~Hz}, 1 \mathrm{H}), 5.12$ $(\mathrm{q}, J=1.3 \mathrm{~Hz}, 1 \mathrm{H}), 3.16-3.07(\mathrm{~m}, 2 \mathrm{H}), 2.97-2.90(\mathrm{~m}, 2 \mathrm{H})$.

${ }^{13} \mathrm{C}$ NMR $\left(126 \mathrm{MHz}, \mathrm{CDCl}_{3}\right) \delta 199.3,162.4(\mathrm{~d}, J=246.5 \mathrm{~Hz}), 146.3,136.8,136.7(\mathrm{~d}, J=3.4$ $\mathrm{Hz}), 133.1,128.6,128.0,127.7$ (d, $J=7.9 \mathrm{~Hz}), 115.2$ (d, $J=21.2 \mathrm{~Hz}), 112.8$ (d, $J=1.4 \mathrm{~Hz})$, 37.3, 29.8.

${ }^{19}$ F NMR $\left(377 \mathrm{MHz}, \mathrm{CDCl}_{3}\right) \delta-114.9$.

IR (ATR) 1672, 1595, 1506, 1447, 1304, 1226, 1184, 1158, 979, 905, 841, 743, 688

HRMS (DART, $\mathrm{M}+\mathrm{H}$ ) Calculated for $\mathrm{C}_{17} \mathrm{H}_{16} \mathrm{~F}_{1} \mathrm{O}_{1} 255.1185$, found 255.1193

MP $71{ }^{\circ} \mathrm{C}$

\section{4-(3-fluoro-4-methoxyphenyl)-1-phenylpent-4-en-1-one (1v)}


<smiles>C=C(CCC(=O)c1ccccc1)c1ccc(OC)c(F)c1</smiles>

Prepared following J. Am. Chem. Soc. 2018, 140, 14647-14654

Prepared on $5.0 \mathrm{mmol}$ scale from the allyl bromide. Obtained a white solid, $427 \mathrm{mg}$ (30\% yield).

${ }^{1} \mathbf{H}$ NMR $\left(500 \mathrm{MHz}, \mathrm{CDCl}_{3}\right) \delta 7.94-7.89(\mathrm{~m}, 2 \mathrm{H}), 7.57-7.52(\mathrm{~m}, 1 \mathrm{H}), 7.46-7.40(\mathrm{~m}, 2 \mathrm{H})$, $7.19(\mathrm{dd}, J=12.7,2.2 \mathrm{~Hz}, 1 \mathrm{H}), 7.15(\mathrm{ddd}, J=8.5,2.3,1.1 \mathrm{~Hz}, 1 \mathrm{H}), 6.91(\mathrm{t}, J=8.6 \mathrm{~Hz}, 1 \mathrm{H}), 5.27$ $(\mathrm{d}, J=1.0 \mathrm{~Hz}, 1 \mathrm{H}), 5.09(\mathrm{q}, J=1.3 \mathrm{~Hz}, 1 \mathrm{H}), 3.89(\mathrm{~s}, 3 \mathrm{H}), 3.16-3.09(\mathrm{~m}, 2 \mathrm{H}), 2.94-2.88(\mathrm{~m}$, $2 \mathrm{H})$.

${ }^{13}$ C NMR (126 MHz, $\left.\mathrm{CDCl}_{3}\right) \delta 199.3,152.2(\mathrm{~d}, J=245.1 \mathrm{~Hz}), 147.1$ (d, $\left.J=10.9 \mathrm{~Hz}\right), 145.7$ (d, $J=1.8 \mathrm{~Hz}), 136.8,133.8(\mathrm{~d}, J=6.2 \mathrm{~Hz}), 133.1,128.6(\mathrm{~d}, J=2.4 \mathrm{~Hz}), 128.0,121.7(\mathrm{~d}, J=3.3$ $\mathrm{Hz}), 113.9$ (d, $J=18.8 \mathrm{~Hz}), 113.13(\mathrm{~d}, J=2.2 \mathrm{~Hz}), 112.3,56.3,37.4,29.5$.

${ }^{19}$ F NMR $\left(377 \mathrm{MHz}, \mathrm{CDCl}_{3}\right) \delta-135.3$.

IR (ATR) 1676, 1618, 1517, 1444, 1307, 1266, 1175, 1026, 898, 825, 747, 687

HRMS (DART, $\mathrm{M}+\mathrm{H}$ ) Calculated for $\mathrm{C}_{18} \mathrm{H}_{18} \mathrm{~F}_{1} \mathrm{O}_{2} 285.1291$, found 285.1298

MP $82{ }^{\circ} \mathrm{C}$

\section{1-phenyl-4-(m-tolyl)pent-4-en-1-one (1w)}<smiles>C=C(CCC(=O)c1ccccc1)c1cccc(C)c1</smiles>

Prepared following J. Am. Chem. Soc. 2018, 140, 14647-14654, consistent with literature spectra Org. Lett., 2018, 20, 1300-1303

${ }^{1} \mathbf{H}$ NMR $\left(500 \mathrm{MHz}, \mathrm{CDCl}_{3}\right) \delta 7.98-7.92(\mathrm{~m}, 2 \mathrm{H}), 7.59-7.52(\mathrm{~m}, 1 \mathrm{H}), 7.49-7.41(\mathrm{~m}, 2 \mathrm{H})$, $7.31-7.22(\mathrm{~m}, 3 \mathrm{H}), 7.16-7.10(\mathrm{~m}, 1 \mathrm{H}), 5.34(\mathrm{~d}, J=1.2 \mathrm{~Hz}, 1 \mathrm{H}), 5.15(\mathrm{q}, J=1.3 \mathrm{~Hz}, 1 \mathrm{H}), 3.18$ $-3.11(\mathrm{~m}, 2 \mathrm{H}), 3.02-2.93(\mathrm{~m}, 2 \mathrm{H}), 2.39(\mathrm{~s}, 3 \mathrm{H})$.

${ }^{13} \mathrm{C}$ NMR $\left(126 \mathrm{MHz}, \mathrm{CDCl}_{3}\right) \delta 199.6,147.5,140.6,138.0,136.9,133.0,128.6,128.4,128.3$, 128.0, 126.9, 123.3, 112.7, 37.6, 29.8, 21.5.

\section{4-(3-methoxyphenyl)-1-phenylpent-4-en-1-one (1x)}<smiles>C=C(CCC(=O)c1ccccc1)c1cccc(OC)c1</smiles> 
Prepared following J. Am. Chem. Soc. 2018, 140, 14647-14654

Prepared on $12 \mathrm{mmol}$ scale from the allyl bromide. Obtained a clear oil, $2.10 \mathrm{~g}$ (66\% yield).

${ }^{1} \mathbf{H}$ NMR $\left(500 \mathrm{MHz}, \mathrm{CDCl}_{3}\right) \delta 7.92(\mathrm{dd}, J=8.4,1.3 \mathrm{~Hz}, 2 \mathrm{H}), 7.57-7.50(\mathrm{~m}, 1 \mathrm{H}), 7.46-7.41$ (m, 2H), $7.26(\mathrm{t}, J=7.9 \mathrm{~Hz}, 1 \mathrm{H}), 7.03(\mathrm{ddd}, J=7.7,1.6,0.9 \mathrm{~Hz}, 1 \mathrm{H}), 6.98(\mathrm{t}, J=2.1 \mathrm{~Hz}, 1 \mathrm{H})$, 6.84 (ddd, $J=8.2,2.6,0.9 \mathrm{~Hz}, 1 \mathrm{H}), 5.34$ (dd, $J=1.2,0.6 \mathrm{~Hz}, 1 \mathrm{H}), 5.15$ (q, $J=1.3 \mathrm{~Hz}, 1 \mathrm{H}$ ), 3.82 (s, 3H), $3.16-3.09(\mathrm{~m}, 2 \mathrm{H}), 2.99-2.92(\mathrm{~m}, 2 \mathrm{H})$.

${ }^{13}$ C NMR $\left(126 \mathrm{MHz}, \mathrm{CDCl}_{3}\right) \delta 199.5,159.7,147.3,142.2,136.9,133.0,129.4,128.5,128.0$, $118.7,113.1,112.9,112.0,55.2,37.5,29.8$.

IR (ATR) 1682, 1597, 1575, 1448, 1285, 1223, 1044, 880, 783, 744, 688

HRMS (DART, $\mathrm{M}+\mathrm{H}$ ) Calculated for $\mathrm{C}_{18} \mathrm{H}_{19} \mathrm{O}_{2} 297.1385$, found 267.1390

\section{4-(3-fluorophenyl)-1-phenylpent-4-en-1-one (1y)}<smiles>C=C(CCC(=O)c1ccccc1)c1cccc(F)c1</smiles>

Prepared following J. Am. Chem. Soc. 2018, 140, 14647-14654

Prepared on $4.6 \mathrm{mmol}$ scale from the allyl bromide. Obtained a white solid, $630 \mathrm{mg}$ (54\% yield).

${ }^{1} \mathbf{H}$ NMR $\left(500 \mathrm{MHz}, \mathrm{CDCl}_{3}\right) \delta 7.95-7.89(\mathrm{~m}, 2 \mathrm{H}), 7.57-7.53(\mathrm{~m}, 1 \mathrm{H}), 7.46-7.42(\mathrm{~m}, 2 \mathrm{H})$, $7.30(\operatorname{td}, J=8.0,6.0 \mathrm{~Hz}, 1 \mathrm{H}), 7.21$ (ddd, $J=7.8,1.7,1.0 \mathrm{~Hz}, 1 \mathrm{H}$ ), 7.13 (ddd, $J=10.4,2.6,1.7$ $\mathrm{Hz}, 1 \mathrm{H}), 6.98(\mathrm{tdd}, J=8.4,2.6,1.0 \mathrm{~Hz}, 1 \mathrm{H}), 5.36(\mathrm{~d}, J=1.3 \mathrm{~Hz}, 1 \mathrm{H}), 5.18(\mathrm{q}, J=1.3 \mathrm{~Hz}, 1 \mathrm{H})$, $3.15-3.10(\mathrm{~m}, 2 \mathrm{H}), 2.97-2.91(\mathrm{~m}, 2 \mathrm{H})$.

${ }^{13}$ C NMR $\left(126 \mathrm{MHz}, \mathrm{CDCl}_{3}\right) \delta$ 199.2, $162.9(\mathrm{~d}, J=245.4 \mathrm{~Hz}), 146.3(\mathrm{~d}, J=2.2 \mathrm{~Hz}), 143.0(\mathrm{~d}, J$ $=7.4 \mathrm{~Hz}), 136.8,133.1,129.9(\mathrm{~d}, J=8.4 \mathrm{~Hz}), 128.6,128.0,121.7(\mathrm{~d}, J=2.8 \mathrm{~Hz}), 114.4(\mathrm{~d}, J=$ $21.2 \mathrm{~Hz}), 113.8,113.1(\mathrm{~d}, J=21.9 \mathrm{~Hz}), 37.3,29.5$.

${ }^{19} \mathbf{F}$ NMR $\left(377 \mathrm{MHz}, \mathrm{CDCl}_{3}\right) \delta-113.3$.

IR (ATR) 1674, 1577, 1520, 1417, 1253, 1188, 895, 794, 676, 653, 599

HRMS (DART, $\mathrm{M}+\mathrm{H}$ ) Calculated for $\mathrm{C}_{17} \mathrm{H}_{16} \mathrm{~F}_{1} \mathrm{O}_{1} 255.1185$, found 255.1188

MP $40{ }^{\circ} \mathrm{C}$

\section{1-phenyl-4-(3-(trifluoromethyl)phenyl)pent-4-en-1-one (1z)}<smiles>C=C(CCC(=O)c1ccccc1)c1cccc(C(F)(F)F)c1</smiles>

Prepared following J. Am. Chem. Soc. 2018, 140, 14647-14654 
Prepared on $6.1 \mathrm{mmol}$ scale from the allyl bromide. Obtained a white solid, $613 \mathrm{mg}$ (51\% yield).

${ }^{1} \mathbf{H}$ NMR $\left(500 \mathrm{MHz}, \mathrm{CDCl}_{3}\right) \delta 7.95-7.90(\mathrm{~m}, 2 \mathrm{H}), 7.68(\mathrm{~s}, 1 \mathrm{H}), 7.61(\mathrm{~d}, J=8.3 \mathrm{~Hz}, 1 \mathrm{H}), 7.58-$ $7.52(\mathrm{~m}, 2 \mathrm{H}), 7.48-7.41(\mathrm{~m}, 3 \mathrm{H}), 5.38(\mathrm{~d}, J=1.2 \mathrm{~Hz}, 1 \mathrm{H}), 5.23(\mathrm{q}, J=1.2 \mathrm{~Hz}, 1 \mathrm{H}), 3.16-3.11$ $(\mathrm{m}, 2 \mathrm{H}), 3.01-2.95(\mathrm{~m}, 2 \mathrm{H})$.

${ }^{13}$ C NMR (126 MHz, $\left.\mathrm{CDCl}_{3}\right) \delta 199.2,146.2,141.6,136.8,133.1,130.8(\mathrm{q}, J=32.1 \mathrm{~Hz}), 129.4$ $(\mathrm{q}, J=1.4 \mathrm{~Hz}), 128.9,128.6,128.0,124.3(\mathrm{q}, J=3.8 \mathrm{~Hz}), 124.1(\mathrm{q}, J=272.4 \mathrm{~Hz}), 122.9(\mathrm{q}, J=$ $3.6 \mathrm{~Hz}), 114.4,37.1,29.5$.

${ }^{19}$ F NMR $\left(377 \mathrm{MHz}, \mathrm{CDCl}_{3}\right) \delta-62.6$.

IR (ATR) 1733, 1578, 1447, 1248, 1153, 1012, 905,827, 720, 662, 629

HRMS (DART, $\mathrm{M}+\mathrm{H}$ ) Calculated for $\mathrm{C}_{18} \mathrm{H}_{16} \mathrm{~F}_{3} \mathrm{O}_{1} 305.1153$, found 305.1153

MP $57^{\circ} \mathrm{C}$

\section{4-(3-chlorophenyl)-1-phenylpent-4-en-1-one (1aa)}<smiles>C=C(CCC(=O)c1ccccc1)c1cccc(Cl)c1</smiles>

Prepared following J. Am. Chem. Soc. 2018, 140, 14647-14654

Prepared on $4.4 \mathrm{mmol}$ scale from the allyl bromide. Obtained a white solid, $411 \mathrm{mg}$ (38\% yield).

${ }^{1} \mathbf{H}$ NMR $\left(500 \mathrm{MHz}, \mathrm{CDCl}_{3}\right) \delta 7.93(\mathrm{dt}, J=8.4,1.6 \mathrm{~Hz}, 2 \mathrm{H}), 7.55(\mathrm{ddt}, J=7.8,6.9,1.3 \mathrm{~Hz}, 1 \mathrm{H})$, $7.47-7.40(\mathrm{~m}, 3 \mathrm{H}), 7.31(\mathrm{~m}, 1 \mathrm{H}), 7.29-7.25(\mathrm{~m}, 2 \mathrm{H}), 5.34(\mathrm{~d}, J=1.3 \mathrm{~Hz}, 1 \mathrm{H}), 5.18(\mathrm{q}, J=1.3$ $\mathrm{Hz}, 1 \mathrm{H}), 3.15-3.10(\mathrm{~m}, 2 \mathrm{H}), 2.98-2.88(\mathrm{~m}, 2 \mathrm{H})$.

${ }^{13} \mathrm{C}$ NMR $\left(126 \mathrm{MHz}, \mathrm{CDCl}_{3}\right) \delta 199.2,146.2,142.6,136.8,134.4,133.1,129.7,128.6,128.0$, 127.6, 126.4, 124.3, 114.0, 37.2, 29.5 .

IR (ATR) 1673, 1592, 1446, 1373, 1308, 1164, 1086, 979, 897, 797, 723, 653, 575

HRMS (DART, M+H) Calculated for $\mathrm{C}_{17} \mathrm{H}_{16} \mathrm{Cl}_{1} \mathrm{O}_{1} 271.0890$, found 271.0894

MP $60{ }^{\circ} \mathrm{C}$

\section{1-phenyl-4-(thiophen-2-yl)pent-4-en-1-one (1ab)}<smiles>C=C(CCC(=O)c1ccccc1)c1cccs1</smiles>

Prepared following Org. Lett., 2018, 20, 1300-1303.

Prepared on $4.4 \mathrm{mmol}$ from the Weinreb amide using organolithium. Obtained $0.95 \mathrm{~g}$ of a white solid $(89 \%$ yield $)$ 
${ }^{1} \mathbf{H}$ NMR $\left(500 \mathrm{MHz}, \mathrm{CDCl}_{3}\right) \delta 8.00-7.93(\mathrm{~m}, 2 \mathrm{H}), 7.60-7.52(\mathrm{~m}, 1 \mathrm{H}), 7.50-7.42(\mathrm{~m}, 2 \mathrm{H})$, $7.19(\mathrm{dd}, J=5.1,1.2 \mathrm{~Hz}, 1 \mathrm{H}), 7.09(\mathrm{dd}, J=3.6,1.2 \mathrm{~Hz}, 1 \mathrm{H}), 6.99(\mathrm{dd}, J=5.1,3.6 \mathrm{~Hz}, 1 \mathrm{H}), 5.45$ (s, 1H), $5.04(\mathrm{~s}, 1 \mathrm{H}), 3.31-3.22(\mathrm{~m}, 2 \mathrm{H}), 3.00-2.89(\mathrm{~m}, 2 \mathrm{H})$.

${ }^{13} \mathrm{C}$ NMR $\left(126 \mathrm{MHz}, \mathrm{CDCl}_{3}\right) \delta 199.2,144.7,140.7,136.8,133.1,128.6,128.0,127.5,124.4$, $123.6,111.5,37.7,29.8$.

IR (ATR) 1615, 1578, 1520, 1417, 1253, 1159, 1025, 1000, 900, 853, 794, 655

HRMS (DART, M+H) Calculated for $\mathrm{C}_{15} \mathrm{H}_{15} \mathrm{O}_{1} \mathrm{~S}_{1} 243.0844$, found 243.0842

MP $62{ }^{\circ} \mathrm{C}$

\section{2,2-dimethyl-1,4-diphenylpent-4-en-1-one (1ac)}<smiles>C=C(CC(C)(C)C(=O)c1ccccc1)c1ccccc1</smiles>

Prepared following Org. Chem. Front. 2018, 5, 3476-3482.

Prepared on $2 \mathrm{mmol}$ from the corresponding ketone. Obtained $255 \mathrm{mg}$ of a clear oil (48\% yield)

${ }^{1} \mathbf{H}$ NMR $\left(500 \mathrm{MHz}, \mathrm{CDCl}_{3}\right) \delta 7.54-7.50(\mathrm{~m}, 2 \mathrm{H}), 7.45-7.40(\mathrm{~m}, 1 \mathrm{H}), 7.38-7.33(\mathrm{~m}, 2 \mathrm{H})$, $7.33-7.30(\mathrm{~m}, 2 \mathrm{H}), 7.30-7.25(\mathrm{~m}, 2 \mathrm{H}), 7.25-7.21(\mathrm{~m}, 1 \mathrm{H}), 5.26(\mathrm{~d}, J=1.6 \mathrm{~Hz}, 1 \mathrm{H}), 5.04$ (dd, $J=1.6,0.9 \mathrm{~Hz}, 1 \mathrm{H}), 3.05(\mathrm{~d}, J=1.1 \mathrm{~Hz}, 2 \mathrm{H}), 1.24(\mathrm{~s}, 6 \mathrm{H})$.

${ }^{13} \mathrm{C}$ NMR $\left(126 \mathrm{MHz}, \mathrm{CDCl}_{3}\right) \delta 208.9,146.1,142.7,139.1,130.6,128.2,127.9,127.6,127.5$, 127.3, 126.7, 117.3, 48.1, 45.2, 26.6 .

IR (ATR) 1671, 1598, 1467, 1444, 1387, 1366, 1237, 1158, 962, 777, 697

HRMS (DART, M+H) Calculated for $\mathrm{C}_{19} \mathrm{H}_{21} \mathrm{O}_{1} 265.1592$, found 265.1592

\section{phenyl(1-(2-phenylallyl)cyclohexyl)methanone (1ad)}<smiles>C=C(CC1(C(=O)c2ccccc2)CCCCC1)c1ccccc1</smiles>

Prepared following Org. Chem. Front. 2018, 5, 3476-3482.

Prepared on $2.5 \mathrm{mmol}$ from the corresponding ketone. Obtained $380 \mathrm{mg}$ of a clear oil (50\% yield)

${ }^{1} \mathbf{H}$ NMR $\left(500 \mathrm{MHz}, \mathrm{CDCl}_{3}\right) \delta 7.61-7.56(\mathrm{~m}, 2 \mathrm{H}), 7.44-7.40(\mathrm{~m}, 1 \mathrm{H}), 7.37-7.32(\mathrm{~m}, 4 \mathrm{H})$, $7.29-7.26(\mathrm{~m}, 2 \mathrm{H}), 7.25-7.20(\mathrm{~m}, 1 \mathrm{H}), 5.25(\mathrm{~d}, J=1.0 \mathrm{~Hz}, 1 \mathrm{H}), 5.05(\mathrm{q}, J=1.0 \mathrm{~Hz}, 1 \mathrm{H}), 3.07$ $(\mathrm{d}, J=1.0 \mathrm{~Hz}, 2 \mathrm{H}), 2.16-2.06(\mathrm{~m}, 2 \mathrm{H}), 1.48-1.32(\mathrm{~m}, 5 \mathrm{H}), 1.23-1.13(\mathrm{~m}, 3 \mathrm{H})$.

${ }^{13} \mathrm{C}$ NMR $\left(126 \mathrm{MHz}, \mathrm{CDCl}_{3}\right) \delta 208.4,145.4,142.8,139.9,130.6,128.2,127.9,127.6,127.3$, $126.7,117.5,53.3,35.1,25.7,23.0$. 
IR (ATR) 2929, 1666, 1627, 1499, 1449, 1327, 1238, 1213, 924, 899, 789, 746, 707

HRMS (DART, M+H) Calculated for $\mathrm{C}_{22} \mathrm{H}_{25} \mathrm{O}_{1} 305.1905$, found 305.1901

\section{(2-chlorophenyl)(1-(2-phenylallyl)cyclopentyl)methanone (1ae)}<smiles>C=C(CC1(C(=O)c2ccccc2Cl)CCCC1)c1ccccc1</smiles>

Prepared following Org. Chem. Front. 2018, 5, 3476-3482.

Prepared on $2 \mathrm{mmol}$ from the corresponding ketone. Obtained $337 \mathrm{mg}$ of a clear oil (52\% yield)

${ }^{1} \mathbf{H}$ NMR $\left(500 \mathrm{MHz}, \mathrm{CDCl}_{3}\right) \delta 7.39-7.35(\mathrm{~m}, 3 \mathrm{H}), 7.34-7.29(\mathrm{~m}, 2 \mathrm{H}), 7.28-7.23(\mathrm{~m}, 2 \mathrm{H})$, $7.16(\mathrm{td}, J=7.6,1.2 \mathrm{~Hz}, 1 \mathrm{H}), 7.00(\mathrm{dd}, J=7.6,1.6 \mathrm{~Hz}, 1 \mathrm{H}), 5.35(\mathrm{~d}, J=1.3 \mathrm{~Hz}, 1 \mathrm{H}), 5.14(\mathrm{q}, J$ $=1.3 \mathrm{~Hz}, 1 \mathrm{H}), 3.05(\mathrm{~d}, J=1.2 \mathrm{~Hz}, 2 \mathrm{H}), 2.12-1.99(\mathrm{~m}, 2 \mathrm{H}), 1.72-1.59(\mathrm{~m}, 6 \mathrm{H})$.

${ }^{13}$ C NMR $\left(126 \mathrm{MHz}, \mathrm{CDCl}_{3}\right) \delta 208.8,146.1,142.4,139.6,130.5,130.2,129.9,128.3,127.5$, 126.6, 126.5, 125.9, 117.1, 60.3, 41.8, 35.9, 24.8 .

IR (ATR) 1687, 1589, 1432, 1268, 1210, 1062, 1014, 935, 905, 768, 703, 640

HRMS (DART, $\mathrm{M}+\mathrm{NH}_{4}$ ) Calculated for $\mathrm{C}_{21} \mathrm{H}_{25} \mathrm{Cl}_{1} \mathrm{~N}_{1} \mathrm{O}_{1} 342.1625$, found 342.1627 
Procedure for catalytic reaction - General Procedure 3<smiles>[R]C(=C)CC([R])([R])C([R])=O</smiles>
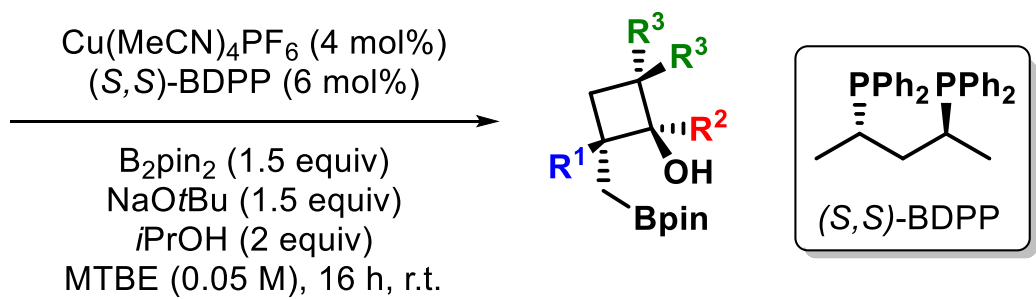

Scheme S2. Catalytic reaction procedure

A 2 dr vial equipped with a stir bar was oven dried and cooled under argon. Copper tetrakisacetonitrile hexafluorophosphate (3.0 mg, $0.008 \mathrm{mmol}, 4 \mathrm{~mol} \%),(S, S)$-BDPP (5.3 mg, $0.012 \mathrm{mmol}, 6 \mathrm{~mol} \%)$, and $\mathrm{NaO} t \mathrm{Bu}(29 \mathrm{mg}, 0.3 \mathrm{mmol}, 1.5$ equiv) were added. MTBE ( $1 \mathrm{~mL})$ was added and the catalyst suspension was stirred for 5 minutes. $B_{2} \operatorname{pin}_{2}(76 \mathrm{mg}, 0.3 \mathrm{mmol}, 1.5$ equiv) was added as a solution in MTBE $(1 \mathrm{~mL})$ and the suspension was stirred for 5 minutes. Next the substrate $(0.2 \mathrm{mmol})$ and $i \mathrm{PrOH}(30 \mu \mathrm{L}, 0.4 \mathrm{mmol}, 2.0$ equiv) were added as a solution in MTBE $(2.0 \mathrm{~mL})$. The reaction was stirred at room temperature for $16 \mathrm{~h}$. Following filtration over a silica pad and removal of the volatile, the $\mathrm{dr}$ was determined by ${ }^{1} \mathrm{H}$ NMR analysis of the crude mixture. The products were isolated by flash column chromatography, typically using acetone/pentane. 


\section{Characterizations of Products}

(1R,2R)-1-methyl-2-phenyl-2-((4,4,5,5-tetramethyl-1,3,2-dioxaborolan-2-

yl)methyl)cyclobutan-1-ol (2a)

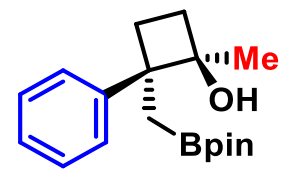

Prepared following General Procedure 3. Product was isolated by flash column chromatography $0->5 \%$ acetone/pentane. Obtained $50 \mathrm{mg}$ of a white solid (83\% yield, 94:6 er).

${ }^{1} \mathbf{H}$ NMR $\left(500 \mathrm{MHz}, \mathrm{CDCl}_{3}\right) \delta 7.37-7.27(\mathrm{~m}, 4 \mathrm{H}), 7.22-7.14(\mathrm{~m}, 1 \mathrm{H}), 2.66$ (dddd, $J=11.6$, 9.2, $6.2,1.0 \mathrm{~Hz}, 1 \mathrm{H}), 2.07(\mathrm{ddd}, J=11.4,8.5,6.2 \mathrm{~Hz}, 1 \mathrm{H}), 1.92(\mathrm{ddd}, J=11.6,8.5,6.3 \mathrm{~Hz}, 1 \mathrm{H})$, 1.82 (ddd, $J=11.4,9.2,6.3 \mathrm{~Hz}, 1 \mathrm{H}), 1.50$ (d, $J=14.7 \mathrm{~Hz}, 1 \mathrm{H}), 1.40$ (s, 3H), 1.31 (s, 1H), 1.27 (d, $J=14.7 \mathrm{~Hz}, 1 \mathrm{H}), 0.94(\mathrm{~s}, 6 \mathrm{H}), 0.94(\mathrm{~s}, 6 \mathrm{H})$.

${ }^{13} \mathrm{C} \mathrm{NMR}\left(126 \mathrm{MHz}, \mathrm{CDCl}_{3}\right) \delta 143.2,128.2,127.5,126.3,82.6,77.9,52.7,33.1,26.2,24.6$, 24.4, 23.7.

IR (ATR) 3419, 2977, 2927, 1349, 4325, 1202, 1142, 965, 845, 695, 563

HRMS (DART, M+NH4) Calculated for $\mathrm{C}_{18} \mathrm{H}_{31} \mathrm{~B}_{1} \mathrm{~N}_{1} \mathrm{O}_{3} 320.2397$, found 320.2404

MP $36{ }^{\circ} \mathrm{C}$

$\boldsymbol{\alpha}_{\boldsymbol{D}}^{\mathbf{2 0}}\left(\mathrm{c}=0.175, \mathrm{CH}_{2} \mathrm{Cl}_{2}\right)-30.1$

HPLC: IC, $4 \%$ IPA/Hex, $0.75 \mathrm{~mL} / \mathrm{min}$

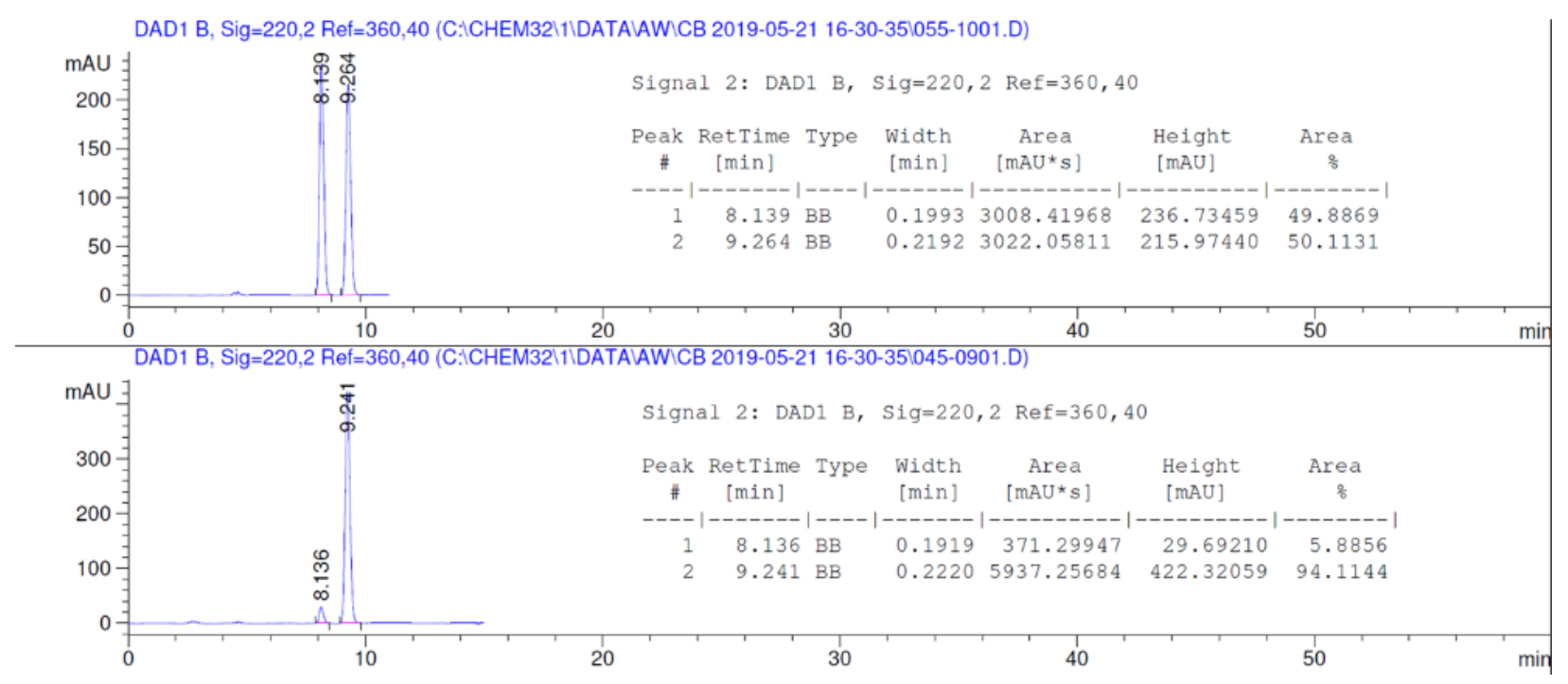


(1S,2R)-1-isopropyl-2-phenyl-2-((4,4,5,5-tetramethyl-1,3,2-dioxaborolan-2yl)methyl)cyclobutan-1-ol (2b)<smiles>[B]C[C@@]1(c2ccccc2)CC[C@@]1(O)C(C)C</smiles>

Prepared following General Procedure 3. Product was isolated by flash column chromatography $0->5 \%$ acetone/pentane. Obtained $66 \mathrm{mg}$ of a clear oil (99\% yield, 95:5 er).

${ }^{1} \mathbf{H}$ NMR $\left(500 \mathrm{MHz}, \mathrm{CDCl}_{3}\right) \delta 7.43-7.38(\mathrm{~m}, 2 \mathrm{H}), 7.32-7.27(\mathrm{~m}, 2 \mathrm{H}), 7.19-7.14(\mathrm{~m}, 1 \mathrm{H})$, $2.76(\mathrm{dtd}, J=11.4,9.2,2.0 \mathrm{~Hz}, 1 \mathrm{H}), 2.11$ (hept, $J=6.7 \mathrm{~Hz}, 1 \mathrm{H}), 2.04(\mathrm{dt}, J=11.4,9.0 \mathrm{~Hz}, 1 \mathrm{H})$, 1.95 (ddd, $J=11.9,9.0,2.9 \mathrm{~Hz}, 1 \mathrm{H}), 1.76$ (ddd, $J=11.9,9.2,2.9 \mathrm{~Hz}, 1 \mathrm{H}), 1.61$ (dd, $J=14.7,2.0$ $\mathrm{Hz}, 1 \mathrm{H}), 1.37$ (d, J = 14.7 Hz, 1H), 1.06 (br, 1H), 0.97 (s, 6H), 0.93 (s, 6H), 0.88 (d, J = 6.7 Hz, $3 \mathrm{H}), 0.84(\mathrm{~d}, J=6.7 \mathrm{~Hz}, 3 \mathrm{H})$.

${ }^{13} \mathrm{C} \mathrm{NMR}\left(126 \mathrm{MHz}, \mathrm{CDCl}_{3}\right) \delta 142.9,128.3,128.0,126.1,83.4,82.7,52.1,32.5,29.5,29.5$, 26.3, 24.7, 24.4, 16.4, 16.3.

IR (ATR) 3469, 2976, 2933, 1461, 1351, 1322, 1279, 1208, 1143, 957, 874, 846, 701

HRMS (DART, $\mathrm{M}+\mathrm{NH}_{4}$ ) Calculated for $\mathrm{C}_{20} \mathrm{H}_{35} \mathrm{~B}_{1} \mathrm{~N}_{1} \mathrm{O}_{3} 348.2710$, found 348.2714

$\boldsymbol{\alpha}_{D}^{\mathbf{2 0}}\left(\mathrm{c}=0.205, \mathrm{CH}_{2} \mathrm{Cl}_{2}\right)-37.4$

HPLC: IC, $4 \%$ IPA/Hex, $0.75 \mathrm{~mL} / \mathrm{min}$

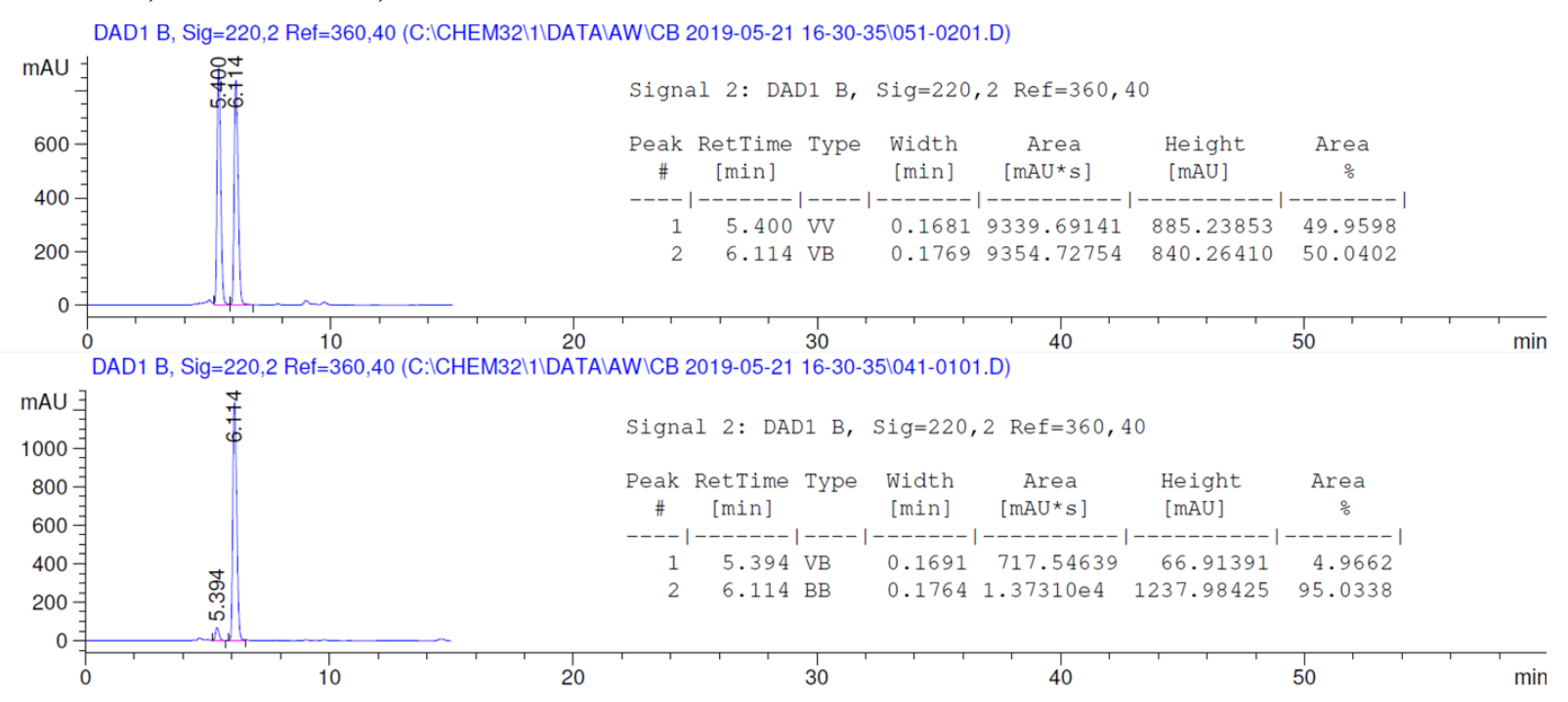


(1S,2R)-1-cyclohexyl-2-phenyl-2-((4,4,5,5-tetramethyl-1,3,2-dioxaborolan-2yl)methyl)cyclobutan-1-ol (2c)

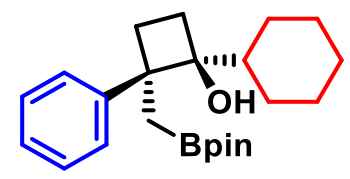

Prepared following General Procedure 3. Product was isolated by flash column chromatography 0 -> 5\% acetone/pentane. Obtained $69 \mathrm{mg}$ of a clear oil (93\% yield, 95.5:4.5 er).

${ }^{1} \mathbf{H}$ NMR $\left(500 \mathrm{MHz}^{\mathrm{CDCl}} 3\right) \delta 7.43-7.37(\mathrm{~m}, 2 \mathrm{H}), 7.29(\mathrm{t}, J=7.8 \mathrm{~Hz}, 2 \mathrm{H}), 7.19-7.11(\mathrm{~m}$, $1 \mathrm{H}), 2.76(\mathrm{dtd}, J=11.6,9.3,2.0 \mathrm{~Hz}, 1 \mathrm{H}), 2.09-2.00(\mathrm{~m}, 1 \mathrm{H}), 1.96(\mathrm{ddd}, J=11.6,9.0,2.3 \mathrm{~Hz}$, $1 \mathrm{H}), 1.74(\mathrm{~m}, 5 \mathrm{H}), 1.66-1.51(\mathrm{~m}, 4 \mathrm{H}), 1.40(\mathrm{~d}, J=14.7 \mathrm{~Hz}, 1 \mathrm{H}), 1.22-1.17(\mathrm{~m}, 1 \mathrm{H}), 1.13-$ $1.07(\mathrm{~m}, 3 \mathrm{H}), 0.98(\mathrm{~s}, 6 \mathrm{H}), 0.93(\mathrm{~s}, 6 \mathrm{H})$.

${ }^{13} \mathrm{C} \mathrm{NMR}\left(126 \mathrm{MHz}, \mathrm{CDCl}_{3}\right) \delta 143.1,128.3,128.0,126.0,83.5,82.7,52.2,43.3,29.0,27.0$, 26.8, 26.7, 26.5, 26.2, 25.6, 24.7, 24.4.

IR (ATR) 3573, 2977, 2928, 2851, 1743, 1447, 1370, 1327, 1279, 1143, 1123, 960, 847, 698, 674

HRMS (ESI, $\mathrm{M}+\mathrm{NH}_{4}$ ) Calculated for $\mathrm{C}_{23} \mathrm{H}_{39} \mathrm{~B}_{1} \mathrm{~N}_{1} \mathrm{O}_{3} 387.3054$, found 387.3054

$\boldsymbol{\alpha}_{\boldsymbol{D}}^{\mathbf{2 0}}\left(\mathrm{c}=0.265, \mathrm{CH}_{2} \mathrm{Cl}_{2}\right)-1.51$

HPLC: IC, 4\% IPA/Hex, $0.75 \mathrm{~mL} / \mathrm{min}$
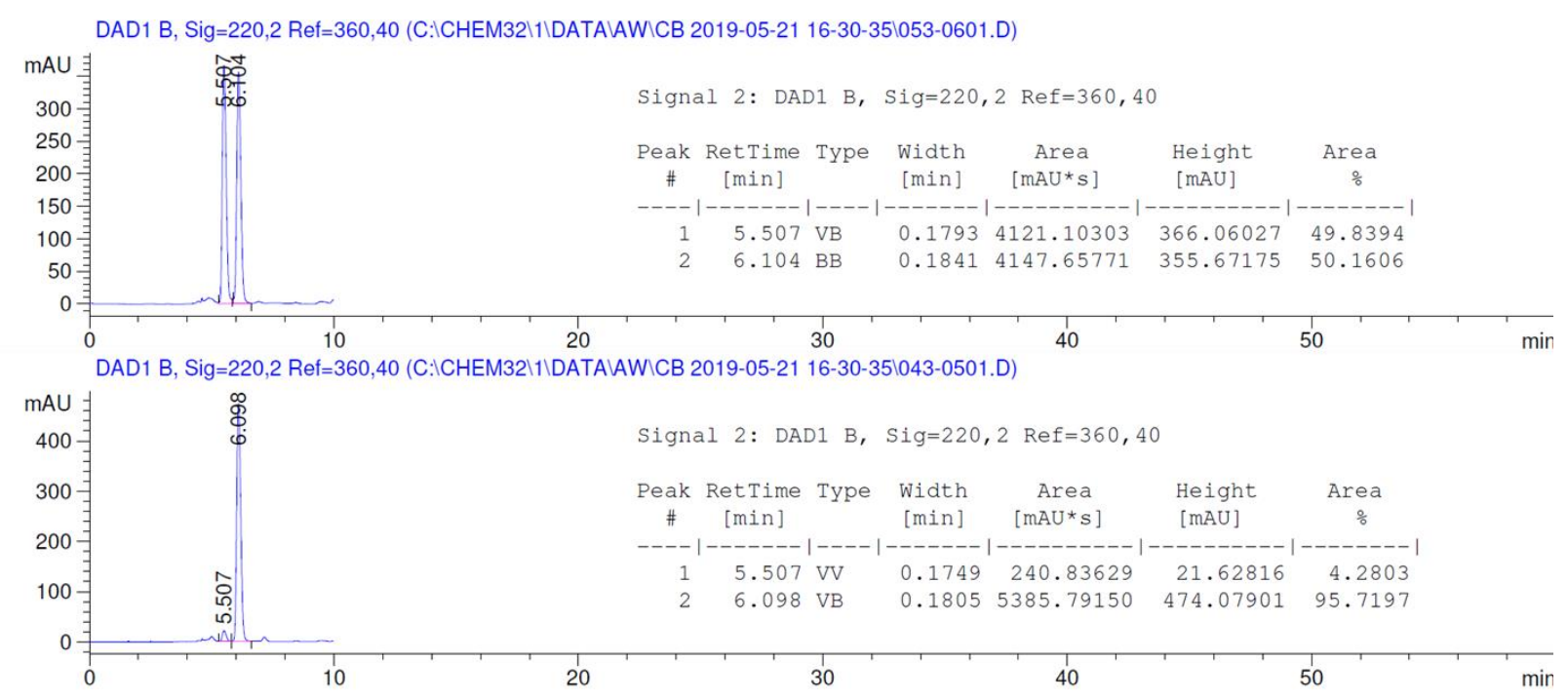
(1S,2R)-1-cyclopropyl-2-phenyl-2-((4,4,5,5-tetramethyl-1,3,2-dioxaborolan-2yl)methyl)cyclobutan-1-ol (2d)<smiles>O[C@@]1(C2CC2)CC[C@@]1(c1ccccc1)C1CC1</smiles>

Prepared following General Procedure 3. Product was isolated by flash column chromatography $0->5 \%$ acetone/pentane. Obtained $58 \mathrm{mg}$ of a clear oil (88\% yield, 95.5:4.5 er).

${ }^{1} \mathbf{H}$ NMR $\left(500 \mathrm{MHz}, \mathrm{CDCl}_{3}\right) \delta 7.33-7.28(\mathrm{~m}, 4 \mathrm{H}), 7.18(\mathrm{p}, J=4.4 \mathrm{~Hz}, 1 \mathrm{H}), 2.74-2.64(\mathrm{~m}$, $1 \mathrm{H}), 2.03-1.92(\mathrm{~m}, 2 \mathrm{H}), 1.69-1.61(\mathrm{~m}, 2 \mathrm{H}), 1.46(\mathrm{~d}, J=14.7 \mathrm{~Hz}, 1 \mathrm{H}), 1.20(\mathrm{ddd}, J=8.2,5.6$, $2.6 \mathrm{~Hz}, 1 \mathrm{H}), 1.14(\mathrm{~s}, 1 \mathrm{H}), 0.96(\mathrm{~s}, 6 \mathrm{H}), 0.95(\mathrm{~s}, 6 \mathrm{H}), 0.55-0.41(\mathrm{~m}, 3 \mathrm{H}), 0.36-0.27(\mathrm{~m}, 1 \mathrm{H})$.

${ }^{13} \mathrm{C}$ NMR $\left(126 \mathrm{MHz}, \mathrm{CDCl}_{3}\right) \delta 143.6,128.2,127.5,126.2,82.6,79.7,53.2,28.7,26.1,24.7$, $24.5,15.6,15.6,1.6,-0.2$.

IR (ATR) 3527, 2978, 1711, 1426, 1355, 1331, 1219, 1145, 1091, 965, 889, 846, 770, 701, 529

HRMS (DART, $\mathrm{M}+\mathrm{NH}_{4}$ ) Calculated for $\mathrm{C}_{20} \mathrm{H}_{33} \mathrm{~B}_{1} \mathrm{~N}_{1} \mathrm{O}_{3} 346.2554$, found 346.2561

$\boldsymbol{\alpha}_{\boldsymbol{D}}^{\mathbf{2 0}}\left(\mathrm{c}=0.170, \mathrm{CH}_{2} \mathrm{Cl}_{2}\right)-34.1$

HPLC: IC, 4\% IPA/Hex, $0.75 \mathrm{~mL} / \mathrm{min}$
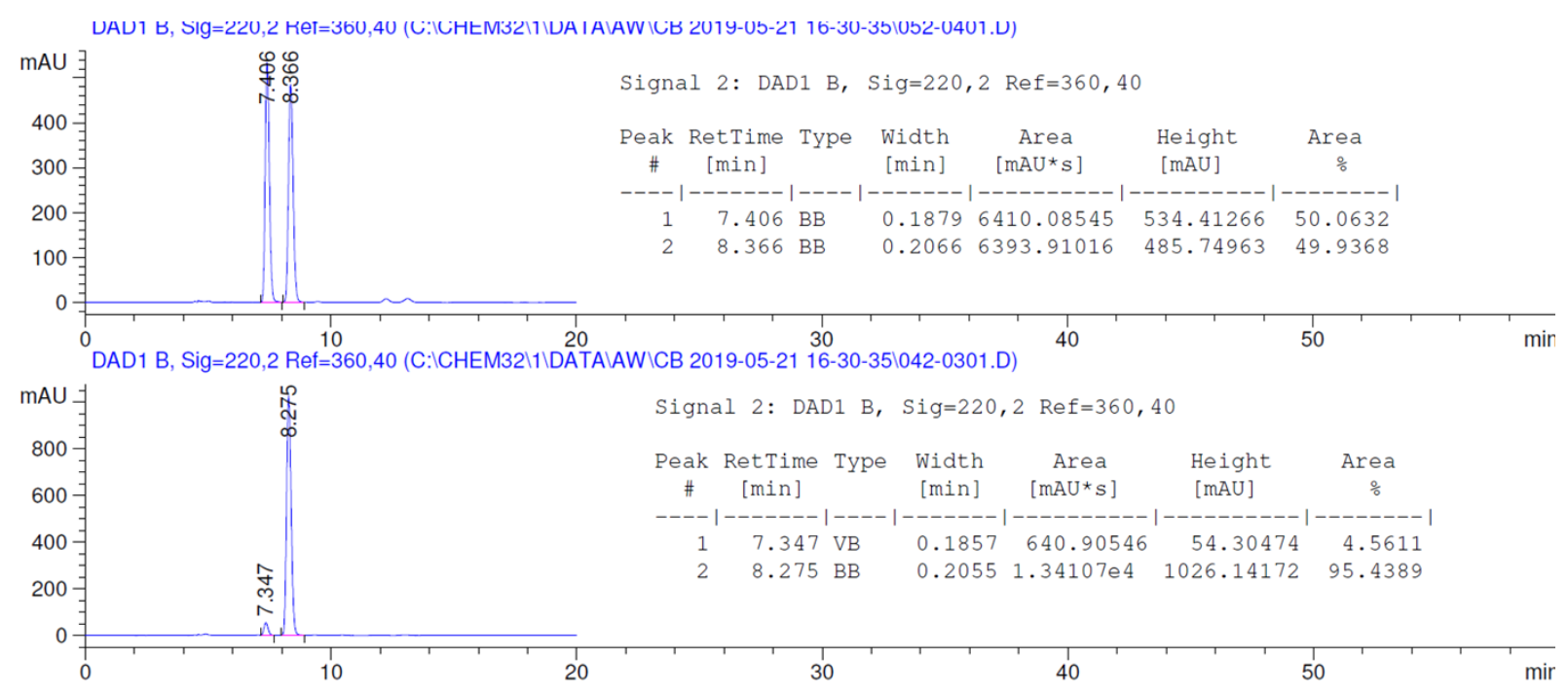
(1S,2R)-1,2-diphenyl-2-((4,4,5,5-tetramethyl-1,3,2-dioxaborolan-2-yl)methyl)cyclobutan-1ol (2e)<smiles>O[C@@]1(c2ccccc2)CCC1(c1ccccc1)c1ccccc1</smiles>

Prepared following General Procedure 3. Product was isolated by flash column chromatography 0 -> 5\% acetone/pentane. Obtained $67 \mathrm{mg}$ of a white solid (92\% yield, 96:4 er).

${ }^{1} \mathbf{H}$ NMR $\left(500 \mathrm{MHz}, \mathrm{CDCl}_{3}\right) \delta 7.53-7.46(\mathrm{~m}, 2 \mathrm{H}), 7.42-7.31(\mathrm{~m}, 6 \mathrm{H}), 7.32-7.24(\mathrm{~m}, 1 \mathrm{H})$, $7.27-7.20(\mathrm{~m}, 1 \mathrm{H}), 3.02-2.91(\mathrm{~m}, 1 \mathrm{H}), 2.14-2.04(\mathrm{~m}, 1 \mathrm{H}), 2.07-1.98$ (m, 2H, overlap), 1.99 (s, 1H), $1.02(\mathrm{~d}, J=15.0 \mathrm{~Hz}, 1 \mathrm{H}), 0.96(\mathrm{dd}, J=15.0,1.3 \mathrm{~Hz}, 1 \mathrm{H}), 0.89(\mathrm{~s}, 6 \mathrm{H}), 0.88(\mathrm{~s}, 6 \mathrm{H})$.

${ }^{13} \mathrm{C}$ NMR $\left(126 \mathrm{MHz}, \mathrm{CDCl}_{3}\right) \delta 142.7,142.6,128.2,127.9,127.8,127.0,126.5,126.5,82.6$, 82.2, 54.0, 29.5, 26.2, 24.6, 24.3.

IR (ATR) 3480, 2936, 1360, 1289, 1270, 1214, 1137, 1019, 958, 847, 780, 606, 588

HRMS (DART, M+NH4) Calculated for $\mathrm{C}_{23} \mathrm{H}_{33} \mathrm{~B}_{1} \mathrm{~N}_{1} \mathrm{O}_{3} 382.2554$, found 382.2549

MP $112{ }^{\circ} \mathrm{C}$

$\boldsymbol{\alpha}_{D}^{\mathbf{2 0}}\left(\mathrm{c}=0.215, \mathrm{CH}_{2} \mathrm{Cl}_{2}\right) 3.1$

HPLC: IC, $4 \%$ IPA/hexane, $0.75 \mathrm{~mL} / \mathrm{min}$

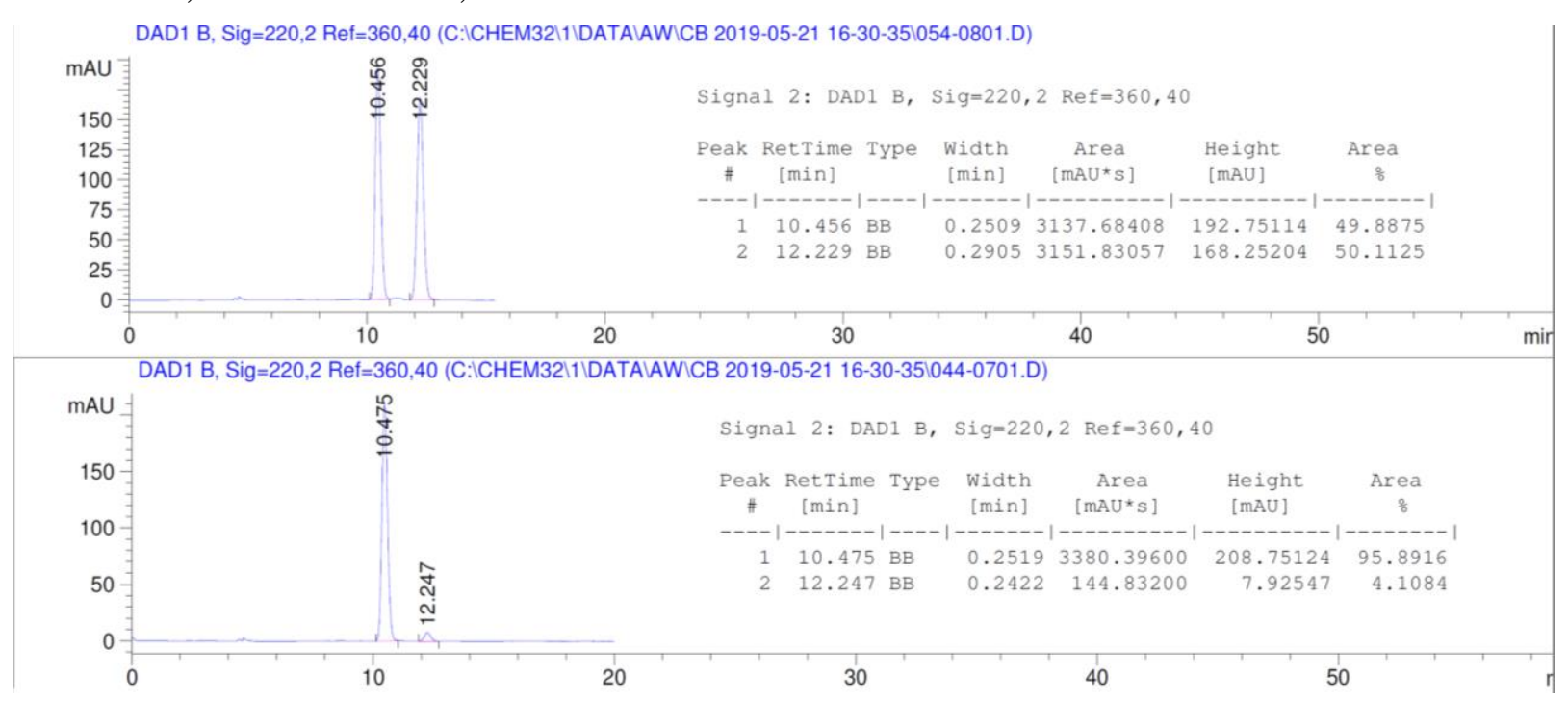


(1S,2R)-2-phenyl-2-((4,4,5,5-tetramethyl-1,3,2-dioxaborolan-2-yl)methyl)-1-(ptolyl)cyclobutan-1-ol (2f)

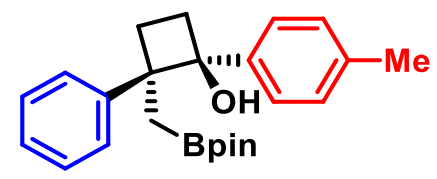

Prepared following General Procedure 3. Product was isolated by flash column chromatography 0 -> 5\% acetone/pentane. Obtained $70 \mathrm{mg}$ of a white solid (93\% yield, 95.5:4.5 er).

${ }^{1} \mathbf{H}$ NMR $\left(500 \mathrm{MHz}, \mathrm{CDCl}_{3}\right) \delta 7.40-7.32(\mathrm{~m}, 6 \mathrm{H}), 7.25-7.20(\mathrm{~m}, 1 \mathrm{H}), 7.18(\mathrm{~d}, J=7.6 \mathrm{~Hz}$, $2 \mathrm{H}), 2.99-2.89(\mathrm{~m}, 2 \mathrm{H}), 2.35(\mathrm{~s}, 3 \mathrm{H}), 2.10-1.96(\mathrm{~m}, 2 \mathrm{H}), 1.94(\mathrm{~s}, 1 \mathrm{H}), 1.01(\mathrm{~d}, J=15.0 \mathrm{~Hz}$, $1 \mathrm{H}), 0.95(\mathrm{dd}, J=15.0,1.3 \mathrm{~Hz}, 1 \mathrm{H}), 0.89(\mathrm{~s}, 6 \mathrm{H}), 0.87$ (s, 6H).

${ }^{13} \mathrm{C}$ NMR $\left(126 \mathrm{MHz}, \mathrm{CDCl}_{3}\right) \delta 142.7,139.8,136.6,128.6,128.2,127.7,126.5,126.4,82.5$, $82.2,53.9,29.5,26.1,24.6,24.3,21.0$.

IR (ATR) 3517, 2977, 2927, 1435, 1348, 1319, 1140, 1032, 955, 845, 803, 742, 701, 575

HRMS (DART, M- $\mathrm{H}_{2} \mathrm{O}+\mathrm{H}$ ) Calculated for $\mathrm{C}_{24} \mathrm{H}_{30} \mathrm{~B}_{1} \mathrm{O}_{2} 361.2334$, found 361.2339

MP $79{ }^{\circ} \mathrm{C}$

$\boldsymbol{\alpha}_{\boldsymbol{D}}^{\mathbf{2 0}}\left(\mathrm{c}=0.400, \mathrm{CH}_{2} \mathrm{Cl}_{2}\right) 23.5$

HPLC: IC, $4 \%$ IPA/Hex, $0.75 \mathrm{~mL} / \mathrm{min}$
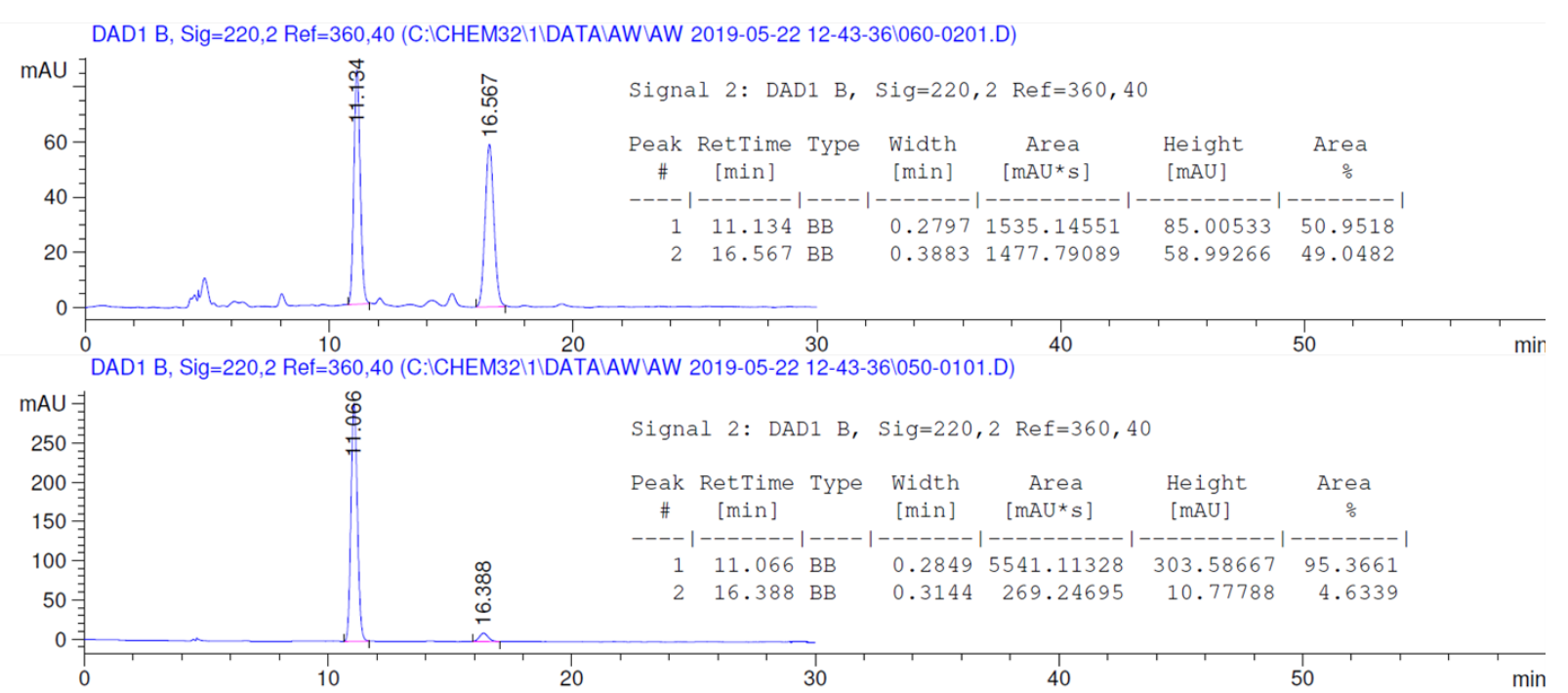
(1S,2R)-1-(4-methoxyphenyl)-2-phenyl-2-((4,4,5,5-tetramethyl-1,3,2-dioxaborolan-2yl)methyl)cyclobutan-1-ol (2g)<smiles>COc1ccc([C@]2(O)CC[C@@]2(CBr)c2ccccc2)cc1</smiles>

Prepared following General Procedure 3. Product was isolated by flash column chromatography 0 -> 5\% acetone/pentane. Obtained $70 \mathrm{mg}$ of a white solid (89\% yield, 95.5:4.5 er).

${ }^{1} \mathbf{H}$ NMR $\left(500 \mathrm{MHz}, \mathrm{CDCl}_{3}\right) \delta 7.41(\mathrm{~d}, J=8.8 \mathrm{~Hz}, 2 \mathrm{H}), 7.38-7.29(\mathrm{~m}, 4 \mathrm{H}), 7.24-7.21(\mathrm{~m}$, $1 \mathrm{H}), 6.91(\mathrm{~d}, J=8.8 \mathrm{~Hz}, 2 \mathrm{H}), 3.82(\mathrm{~s}, 3 \mathrm{H}), 2.98-2.87(\mathrm{~m}, 2 \mathrm{H}), 2.10-1.96(\mathrm{~m}, 2 \mathrm{H}), 1.95-1.90$ (m, 1H), $1.00(\mathrm{~d}, J=15.0 \mathrm{~Hz}, 1 \mathrm{H}), 0.95$ (dd, $J=15.0,1.2 \mathrm{~Hz}, 1 \mathrm{H}), 0.89$ (s, 6H), 0.87 (s, 6H).

${ }^{13} \mathrm{C} \mathrm{NMR}\left(126 \mathrm{MHz}, \mathrm{CDCl}_{3}\right) \delta 158.7,142.7,135.1,128.2,127.7,127.7,126.5,113.3,82.5$, 82.1, 55.3, 54.0, 29.7, 26.1, 24.6, 24.3.8939

IR (ATR) 3432, 2976, 2933, 1610, 1510, 1354, 1290, 1251, 1141, 1103, 1031, 954, 831, 701, 583

HRMS (DART, M-H $\mathrm{H}_{2} \mathrm{O}+\mathrm{H}$ ) Calculated for $\mathrm{C}_{24} \mathrm{H}_{30} \mathrm{~B}_{1} \mathrm{O}_{3} 377.2288$, found 377.2291

MP $91{ }^{\circ} \mathrm{C}$

$\boldsymbol{\alpha}_{\boldsymbol{D}}^{\mathbf{2 0}}\left(\mathrm{c}=0.17, \mathrm{CH}_{2} \mathrm{Cl}_{2}\right) 32.2$

HPLC: IA, 6\% IPA/Hex, $1 \mathrm{~mL} / \mathrm{min}$

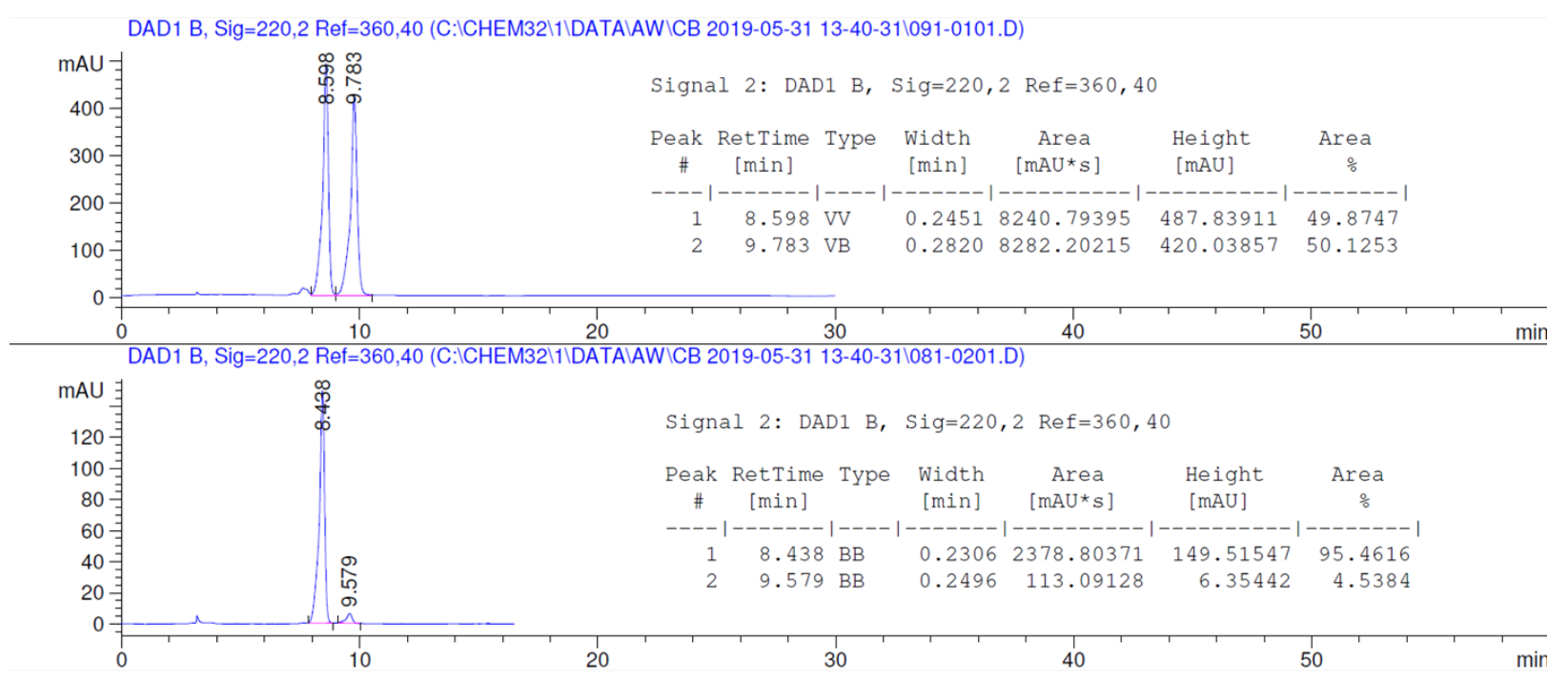


(1S,2R)-1-(4-fluorophenyl)-2-phenyl-2-((4,4,5,5-tetramethyl-1,3,2-dioxaborolan-2yl)methyl)cyclobutan-1-ol (2h)

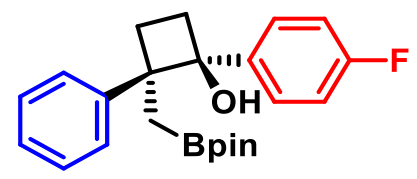

Prepared following General Procedure 3. Product was isolated by flash column chromatography 0 -> 5\% acetone/pentane. Obtained $69 \mathrm{mg}$ of a white solid (89\% yield, 96.5:3.5 er).

${ }^{1} \mathbf{H}$ NMR $\left(500 \mathrm{MHz}, \mathrm{CDCl}_{3}\right) \delta 7.49-7.43(\mathrm{~m}, 2 \mathrm{H}), 7.39-7.34(\mathrm{~m}, 2 \mathrm{H}), 7.33-7.29(\mathrm{~m}, 2 \mathrm{H})$, $7.26-7.22(\mathrm{~m}, 1 \mathrm{H}), 7.08-7.03(\mathrm{~m}, 2 \mathrm{H}), 2.99-2.86(\mathrm{~m}, 2 \mathrm{H}), 2.11-1.99(\mathrm{~m}, 2 \mathrm{H}), 1.97(\mathrm{~s}, 1 \mathrm{H})$, $0.97(\mathrm{~d}, J=14.9 \mathrm{~Hz}, 1 \mathrm{H}), 0.93(\mathrm{~d}, J=14.9 \mathrm{~Hz}, 1 \mathrm{H}), 0.89$ (s, 6H), 0.87 (s, 6H).

${ }^{13} \mathrm{C} \mathrm{NMR}\left(126 \mathrm{MHz}, \mathrm{CDCl}_{3}\right) \delta 162.0(\mathrm{~d}, J=245.5 \mathrm{~Hz}), 142.3,138.6(\mathrm{~d}, J=3.1 \mathrm{~Hz}), 128.3$, $128.2(\mathrm{~d}, J=8.0 \mathrm{~Hz}), 127.7,126.7,114.6(\mathrm{~d}, J=21.1 \mathrm{~Hz}), 82.6,81.8,54.1(\mathrm{~d}, J=1.1 \mathrm{~Hz}), 29.9$, 26.1, 24.6, 24.3.

${ }^{19}$ F NMR $\left(377 \mathrm{MHz}, \mathrm{CDCl}_{3}\right) \delta-116.2$.

IR (ATR) 3527, 2975, 1600, 1509, 1352, 1323, 1219, 1139, 1029, 965, 833, 703, 585

HRMS (DART, $\mathrm{M}+\mathrm{NH}_{4}$ ) Calculated for $\mathrm{C}_{23} \mathrm{H}_{32} \mathrm{~B}_{1} \mathrm{~F}_{1} \mathrm{~N}_{1} \mathrm{O}_{3} 400.2459$, found 400.2460

MP $99{ }^{\circ} \mathrm{C}$

$\boldsymbol{\alpha}_{\boldsymbol{D}}^{\mathbf{2 0}}\left(\mathrm{c}=0.195, \mathrm{CH}_{2} \mathrm{Cl}_{2}\right) 4.3$

HPLC: IA, 4\% IPA/Hex, $0.75 \mathrm{~mL} / \mathrm{min}$

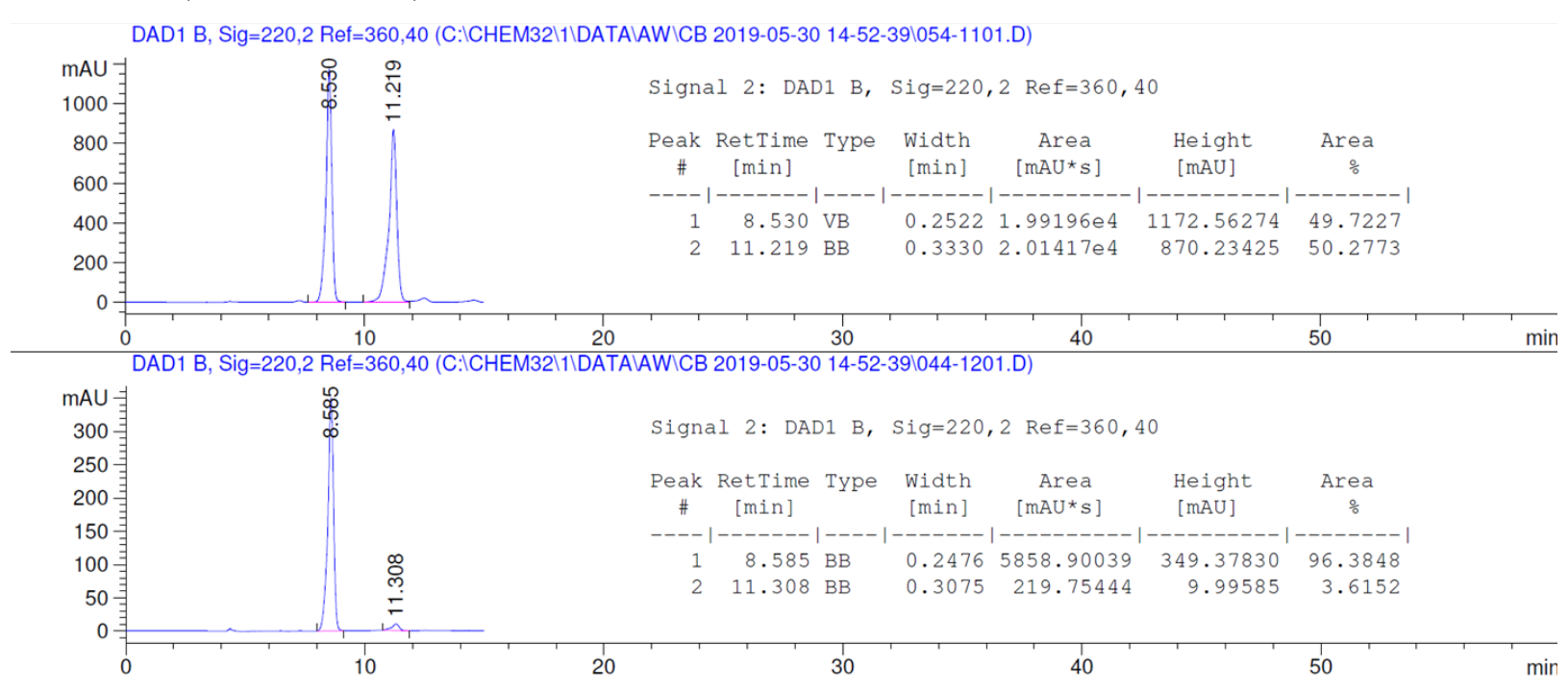


(1S,2R)-2-phenyl-2-((4,4,5,5-tetramethyl-1,3,2-dioxaborolan-2-yl)methyl)-1-(4(trifluoromethyl)phenyl)cyclobutan-1-ol (2i)<smiles>O[C@]1(c2ccccc2)CC[C@@]1(C[Pb])c1ccc(C(F)(F)F)cc1</smiles>

Prepared following General Procedure 3. Product was isolated by flash column chromatography 0 -> 5\% acetone/pentane. Obtained $34 \mathrm{mg}$ of a white solid (39\% yield, 99:1 er).

${ }^{1} \mathbf{H}$ NMR $\left(500 \mathrm{MHz}, \mathrm{CDCl}_{3}\right) \delta 7.66-7.59(\mathrm{~m}, 4 \mathrm{H}), 7.40-7.35(\mathrm{~m}, 2 \mathrm{H}), 7.33-7.28(\mathrm{~m}, 2 \mathrm{H})$, $7.28-7.24(\mathrm{~m}, 1 \mathrm{H}), 3.02-2.90(\mathrm{~m}, 2 \mathrm{H}), 2.14-2.05(\mathrm{~m}, 2 \mathrm{H}), 2.03(\mathrm{~s}, 1 \mathrm{H}), 0.98(\mathrm{~d}, J=14.9 \mathrm{~Hz}$, $1 \mathrm{H}), 0.92-0.90(\mathrm{~m}, 1 \mathrm{H}), 0.89(\mathrm{~s}, 6 \mathrm{H}), 0.87(\mathrm{~s}, 6 \mathrm{H})$.

${ }^{13}$ C NMR (126 MHz, $\left.\mathrm{CDCl}_{3}\right) \delta 146.7$ (q, $\left.J=1.1 \mathrm{~Hz}\right), 141.8,129.2$ (q, $\left.J=32.4 \mathrm{~Hz}\right), 128.4$, $127.7,126.9,126.9,124.8(\mathrm{q}, J=3.8 \mathrm{~Hz}), 124.1(\mathrm{q}, J=271.6 \mathrm{~Hz}), 82.7,81.7,54.2,29.8,26.2$, 24.5, 24.3.

${ }^{19}$ F NMR $\left(377 \mathrm{MHz}, \mathrm{CDCl}_{3}\right) \delta-62.4$.

IR (ATR) 3527, 2974, 1358, 1327, 1291, 1165, 1124, 1070, 1030, 966, 834, 700, 610

HRMS (DART, $\mathrm{M}+\mathrm{NH}_{4}$ ) Calculated for $\mathrm{C}_{24} \mathrm{H}_{32} \mathrm{~B}_{1} \mathrm{~F}_{3} \mathrm{~N}_{1} \mathrm{O}_{3} 450.2427$, found 450.2433

MP $106^{\circ} \mathrm{C}$

$\boldsymbol{\alpha}_{D}^{\mathbf{2 0}}\left(\mathrm{c}=0.120, \mathrm{CH}_{2} \mathrm{Cl}_{2}\right) 16.7$

HPLC: IA, 4\% IPA/Hex, $0.75 \mathrm{~mL} / \mathrm{min}$

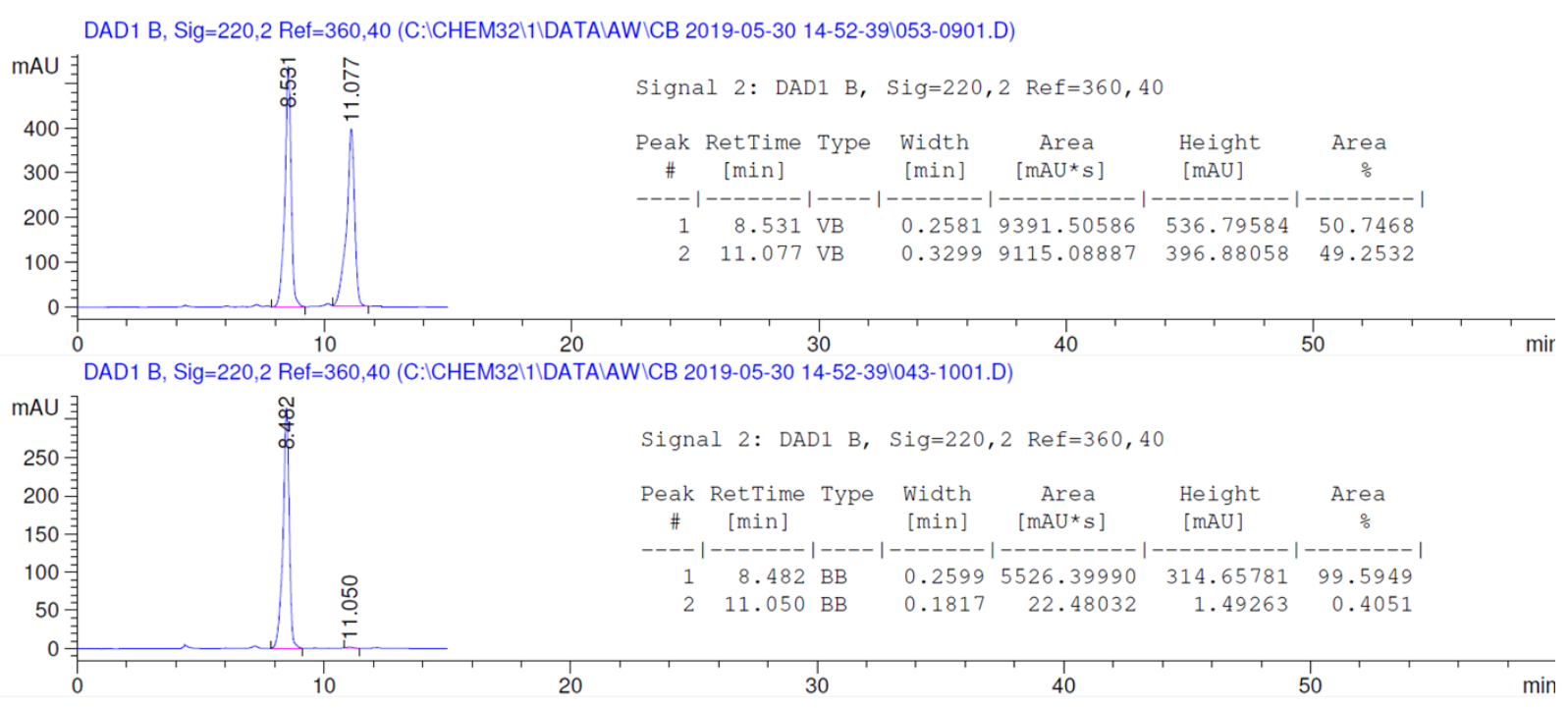


(1S,2R)-2-phenyl-2-((4,4,5,5-tetramethyl-1,3,2-dioxaborolan-2-yl)methyl)-1-(mtolyl)cyclobutan-1-ol (2j)

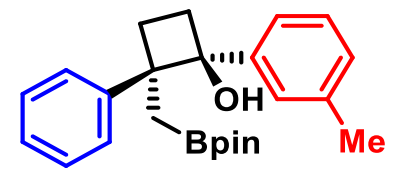

Prepared following General Procedure 3. Product was isolated by flash column chromatography $0->5 \%$ acetone/pentane. Obtained $66 \mathrm{mg}$ of a white solid (87\% yield, 96.5:3.5 er).

${ }^{1} \mathbf{H}$ NMR $\left(500 \mathrm{MHz}, \mathrm{CDCl}_{3}\right) \delta 7.38-7.34(\mathrm{~m}, 4 \mathrm{H}), 7.31-7.26(\mathrm{~m}, 3 \mathrm{H}), 7.26-7.22(\mathrm{~m}, 1 \mathrm{H})$, 7.09 (dtd, $J=6.7,1.7,0.8 \mathrm{~Hz}, 1 \mathrm{H}), 3.01-2.88(\mathrm{~m}, 2 \mathrm{H}), 2.39(\mathrm{~s}, 3 \mathrm{H}), 2.10-1.97(\mathrm{~m}, 2 \mathrm{H}), 1.95$ (s, 1H), 0.99 (s, 2H), $0.89(\mathrm{~s}, 6 \mathrm{H}), 0.87(\mathrm{~s}, 6 \mathrm{H})$.

${ }^{13} \mathbf{C ~ N M R}\left(126 \mathrm{MHz}, \mathrm{CDCl}_{3}\right) \delta 142.7,142.6,137.3,128.2,127.8,127.8,127.8,127.2,126.5$, 123.6, 82.6, 82.2, 54.0, 29.6, 26.2, 24.6, 24.3, 21.7.

IR (ATR) 3529, 2975, 1447, 1349, 1328, 1138, 961, 845, 787, 742, 701, 677, 596

HRMS (DART, M+NH 4 ) Calculated for $\mathrm{C}_{24} \mathrm{H}_{35} \mathrm{~B}_{1} \mathrm{~N}_{1} \mathrm{O}_{3} 396.2710$, found 396.2711

MP $96{ }^{\circ} \mathrm{C}$

$\boldsymbol{\alpha}_{D}^{\mathbf{2 0}}\left(\mathrm{c}=0.160, \mathrm{CH}_{2} \mathrm{Cl}_{2}\right) 1.26$

HPLC: IA, $4 \%$ IPA/Hex, $0.75 \mathrm{~mL} / \mathrm{min}$

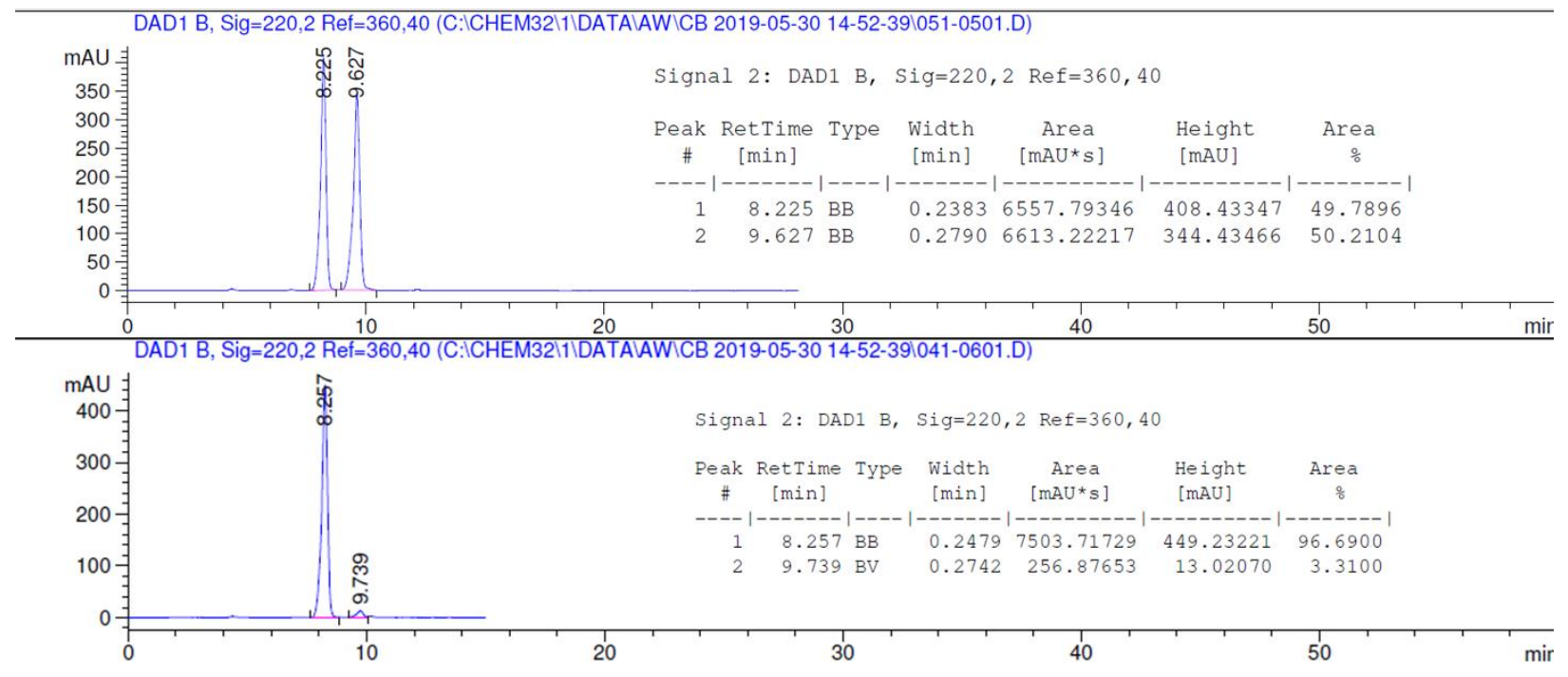


(1S,2R)-1-(3-methoxyphenyl)-2-phenyl-2-((4,4,5,5-tetramethyl-1,3,2-dioxaborolan-2yl)methyl)cyclobutan-1-ol (2k)

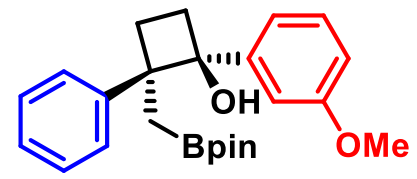

Prepared following General Procedure 3. Product was isolated by flash column chromatography $0->6 \%$ acetone/pentane. Obtained $69 \mathrm{mg}$ of a white solid (87\% yield, 96.5:3.5 er).

${ }^{1} \mathbf{H}$ NMR $\left(500 \mathrm{MHz} \mathrm{CDCl}_{3}\right) \delta 7.39-7.33(\mathrm{~m}, 4 \mathrm{H}), 7.29(\mathrm{t}, J=7.9 \mathrm{~Hz}, 1 \mathrm{H}), 7.23(\mathrm{tt}, J=4.7,3.5$ $\mathrm{Hz}, 1 \mathrm{H}), 7.07$ (ddd, $J=7.9,1.7,0.9 \mathrm{~Hz}, 1 \mathrm{H}), 7.04(\mathrm{dd}, J=2.6,1.7 \mathrm{~Hz}, 1 \mathrm{H}), 6.82$ (ddd, $J=8.2$, $2.6,0.9 \mathrm{~Hz}, 1 \mathrm{H}), 3.82(\mathrm{~s}, 3 \mathrm{H}), 3.00-2.87(\mathrm{~m}, 2 \mathrm{H}), 2.11-1.98(\mathrm{~m}, 2 \mathrm{H}), 1.96(\mathrm{~s}, 1 \mathrm{H}), 1.03(\mathrm{~d}, J=$ $15.0 \mathrm{~Hz}, 1 \mathrm{H}), 0.99$ (d, $J=15.0 \mathrm{~Hz}, 1 \mathrm{H}), 0.89$ (s, 6H), 0.87 (s, 6H).

${ }^{13} \mathrm{C}$ NMR $\left(126 \mathrm{MHz}, \mathrm{CDCl}_{3}\right) \delta 159.4,144.5,142.5,128.8,128.2,127.8,126.6,119.0,112.6$, $112.3,82.6,82.2,55.2,54.0,29.6,26.1,24.6,24.3$.

IR (ATR) 3449, 2982, 1594, 1461, 1351, 1290, 1267, 1202, 1135, 1036, 958, 879, 792, 700, 580

HRMS (DART, M- $\mathrm{H}_{2} \mathrm{O}+\mathrm{H}$ ) Calculated for $\mathrm{C}_{24} \mathrm{H}_{30} \mathrm{~B}_{1} \mathrm{O}_{3} 377.2288$, found 377.2292

MP $125^{\circ} \mathrm{C}$

$\boldsymbol{\alpha}_{\boldsymbol{D}}^{\mathbf{2 0}}\left(\mathrm{c}=0.225, \mathrm{CH}_{2} \mathrm{Cl}_{2}\right)-3.0$

HPLC: IC, $4 \%$ IPA/Hex, $0.75 \mathrm{~mL} / \mathrm{min}$

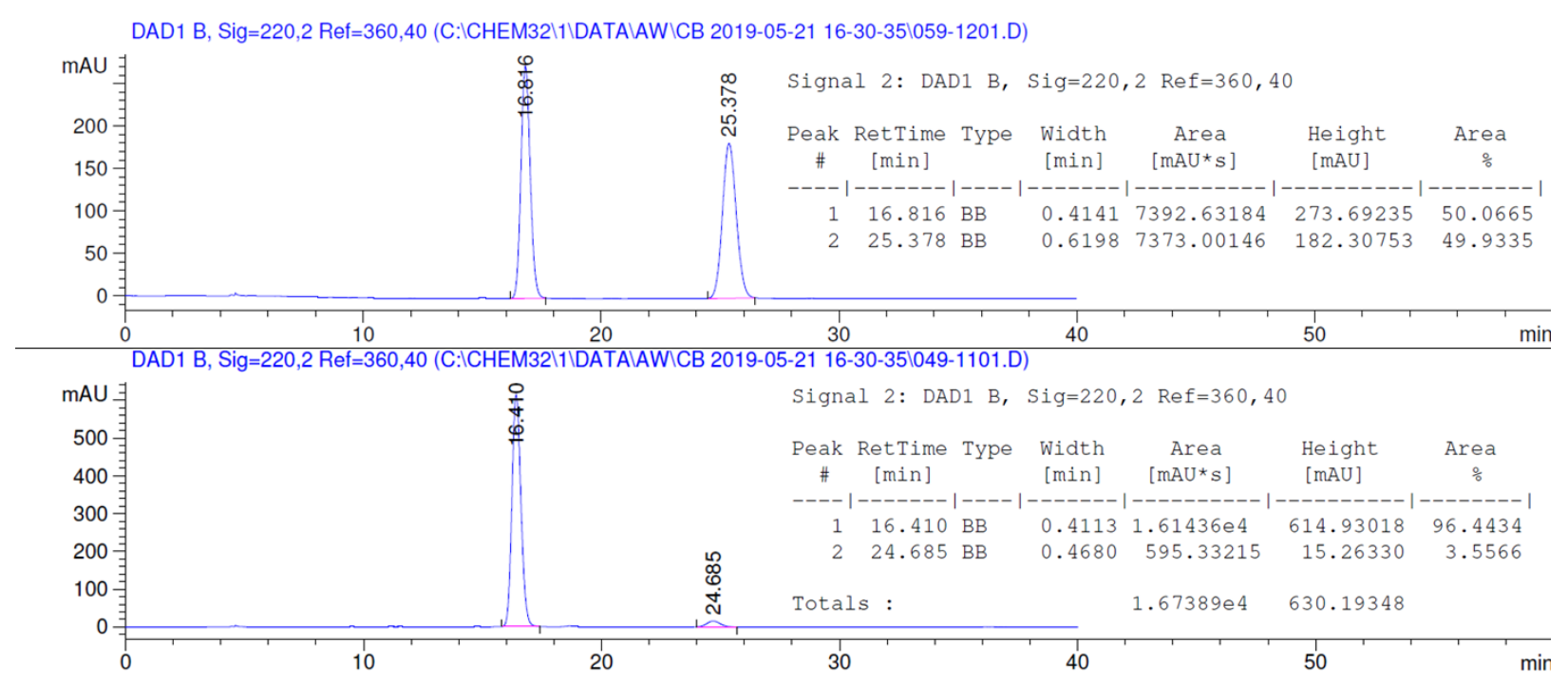


(1S,2R)-1-(3-fluorophenyl)-2-phenyl-2-((4,4,5,5-tetramethyl-1,3,2-dioxaborolan-2yl)methyl)cyclobutan-1-ol (2l)

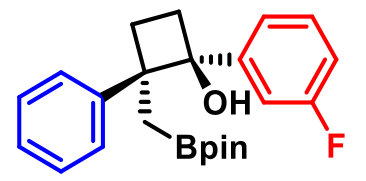

Prepared following General Procedure 3. Product was isolated by flash column chromatography $0->5 \%$ acetone/pentane. Obtained $64 \mathrm{mg}$ of a white solid (84\% yield, 97.5:2.5 er).

${ }^{1} \mathbf{H}$ NMR $\left(500 \mathrm{MHz}, \mathrm{CDCl}_{3}\right) \delta 7.40-7.30(\mathrm{~m}, 5 \mathrm{H}), 7.28-7.22(\mathrm{~m}, 2 \mathrm{H}), 7.20$ (ddd, $J=10.7$, 2.6, $1.7 \mathrm{~Hz}, 1 \mathrm{H}), 6.98(\mathrm{tdd}, J=8.3,2.6,1.1 \mathrm{~Hz}, 1 \mathrm{H}), 2.95(\mathrm{dtd}, J=10.2,7.9,1.3 \mathrm{~Hz}, 1 \mathrm{H}), 2.88$ $(\mathrm{dt}, J=12.0,7.9 \mathrm{~Hz}, 1 \mathrm{H}), 2.12-2.01(\mathrm{~m}, 2 \mathrm{H}), 2.00(\mathrm{~s}, 1 \mathrm{H}), 1.01(\mathrm{~d}, J=14.9 \mathrm{~Hz}, 1 \mathrm{H}), 0.96(\mathrm{dd}$, $J=14.9,1.3 \mathrm{~Hz}, 1 \mathrm{H}), 0.89(\mathrm{~s}, 6 \mathrm{H}), 0.87(\mathrm{~s}, 6 \mathrm{H})$.

${ }^{13}$ C NMR $\left(126 \mathrm{MHz}, \mathrm{CDCl}_{3}\right) \delta 162.8(\mathrm{~d}, J=244.9 \mathrm{~Hz}), 145.7(\mathrm{~d}, J=6.6 \mathrm{~Hz}), 142.1,129.3(\mathrm{~d}, J$ $=8.1 \mathrm{~Hz}), 128.4,127.7,126.8,122.2(\mathrm{~d}, J=2.8 \mathrm{~Hz}), 113.9(\mathrm{~d}, J=10.7 \mathrm{~Hz}), 113.7(\mathrm{~d}, J=11.7$ $\mathrm{Hz}), 82.7,81.8$ (d, $J=1.7 \mathrm{~Hz}), 54.2,29.9,26.1,24.6,24.3$.

${ }^{19}$ F NMR $\left(377 \mathrm{MHz}, \mathrm{CDCl}_{3}\right) \delta-113.6$.

IR (ATR) 3525, 2977, 1585, 1434, 1351, 1336, 1265, 1138, 963, 878, 846, 783, 702, 677

HRMS (DART, $\mathrm{M}+\mathrm{NH}_{4}$ ) Calculated for $\mathrm{C}_{23} \mathrm{H}_{32} \mathrm{~B}_{1} \mathrm{~F}_{1} \mathrm{~N}_{1} \mathrm{O}_{3} 400.2459$, found 400.2467

MP $76^{\circ} \mathrm{C}$

$\boldsymbol{\alpha}_{\boldsymbol{D}}^{\mathbf{2 0}}\left(\mathrm{c}=0.30, \mathrm{CH}_{2} \mathrm{Cl}_{2}\right) 2.9$

HPLC: IC, $4 \%$ IPA/Hex, $0.75 \mathrm{~mL} / \mathrm{min}$

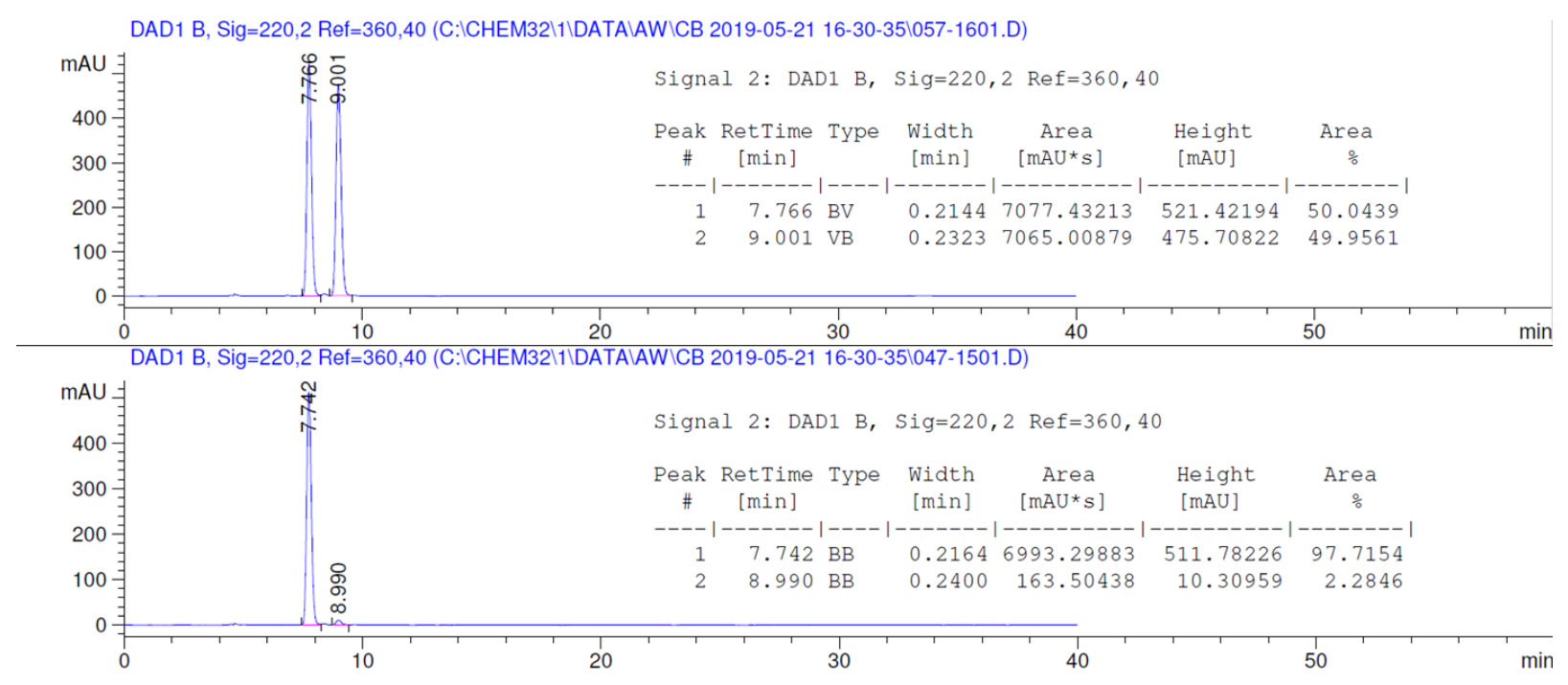


(1S,2R)-2-phenyl-2-((4,4,5,5-tetramethyl-1,3,2-dioxaborolan-2-yl)methyl)-1-(3(trifluoromethyl)phenyl)cyclobutan-1-ol (2m)

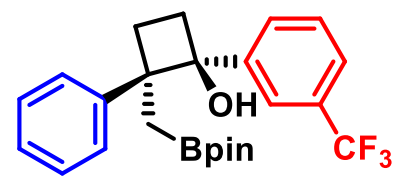

Prepared following General Procedure 3. Product was isolated by flash column chromatography $0->5 \%$ acetone/pentane. Obtained $46 \mathrm{mg}$ of a white solid (53\% yield, 99:1 er).

${ }^{1} \mathbf{H}$ NMR $\left(500 \mathrm{MHz}, \mathrm{CDCl}_{3} \delta 7.76(\mathrm{~s}, 1 \mathrm{H}), 7.69(\mathrm{~d}, J=7.7 \mathrm{~Hz}, 1 \mathrm{H}), 7.55(\mathrm{~d}, J=7.7 \mathrm{~Hz}, 1 \mathrm{H})\right.$, $7.49(\mathrm{t}, J=7.7 \mathrm{~Hz}, 1 \mathrm{H}), 7.40-7.35(\mathrm{~m}, 2 \mathrm{H}), 7.33-7.29(\mathrm{~m}, 2 \mathrm{H}), 7.29-7.24(\mathrm{~m}, 1 \mathrm{H}), 3.02-$ $2.88(\mathrm{~m}, 2 \mathrm{H}), 2.14-2.02(\mathrm{~m}, 3 \mathrm{H}), 0.95(\mathrm{~d}, J=14.9 \mathrm{~Hz}, 1 \mathrm{H}), 0.90(\mathrm{~d}, J=14.9 \mathrm{~Hz}, 1 \mathrm{H}), 0.88(\mathrm{~s}$, $6 \mathrm{H}), 0.87(\mathrm{~s}, 6 \mathrm{H})$.

${ }^{13}$ C NMR (126 MHz, $\left.\mathrm{CDCl}_{3}\right) \delta 143.8,141.8,130.3$ (q, $\left.J=32.1 \mathrm{~Hz}\right), 129.9,128.4,128.3,127.7$, $126.9,124.3(\mathrm{q}, J=272.5 \mathrm{~Hz}), 123.9(\mathrm{q}, J=3.6 \mathrm{~Hz}), 123.4,82.7,81.7,54.2,29.8,26.1,24.5$, 24.3 .

${ }^{19}$ F NMR $\left(377 \mathrm{MHz}, \mathrm{CDCl}_{3}\right) \delta-62.4$.

IR (ATR) 3521, 2983, 1354, 1333, 1249, 1158, 1121, 1075, 1040, 966, 906, 888, 844, 809, 701, 671

HRMS (DART, $\mathrm{M}+\mathrm{NH}_{4}$ ) Calculated for $\mathrm{C}_{24} \mathrm{H}_{32} \mathrm{~B}_{1} \mathrm{~F}_{3} \mathrm{~N}_{1} \mathrm{O}_{3} 450.2427$, found 450.2435

MP $115^{\circ} \mathrm{C}$

$\boldsymbol{\alpha}_{\boldsymbol{D}}^{\mathbf{2 0}}\left(\mathrm{c}=0.17, \mathrm{CH}_{2} \mathrm{Cl}_{2}\right) 3.5$

HPLC: IA, 4\% IPA/Hex, $0.75 \mathrm{~mL} / \mathrm{min}$
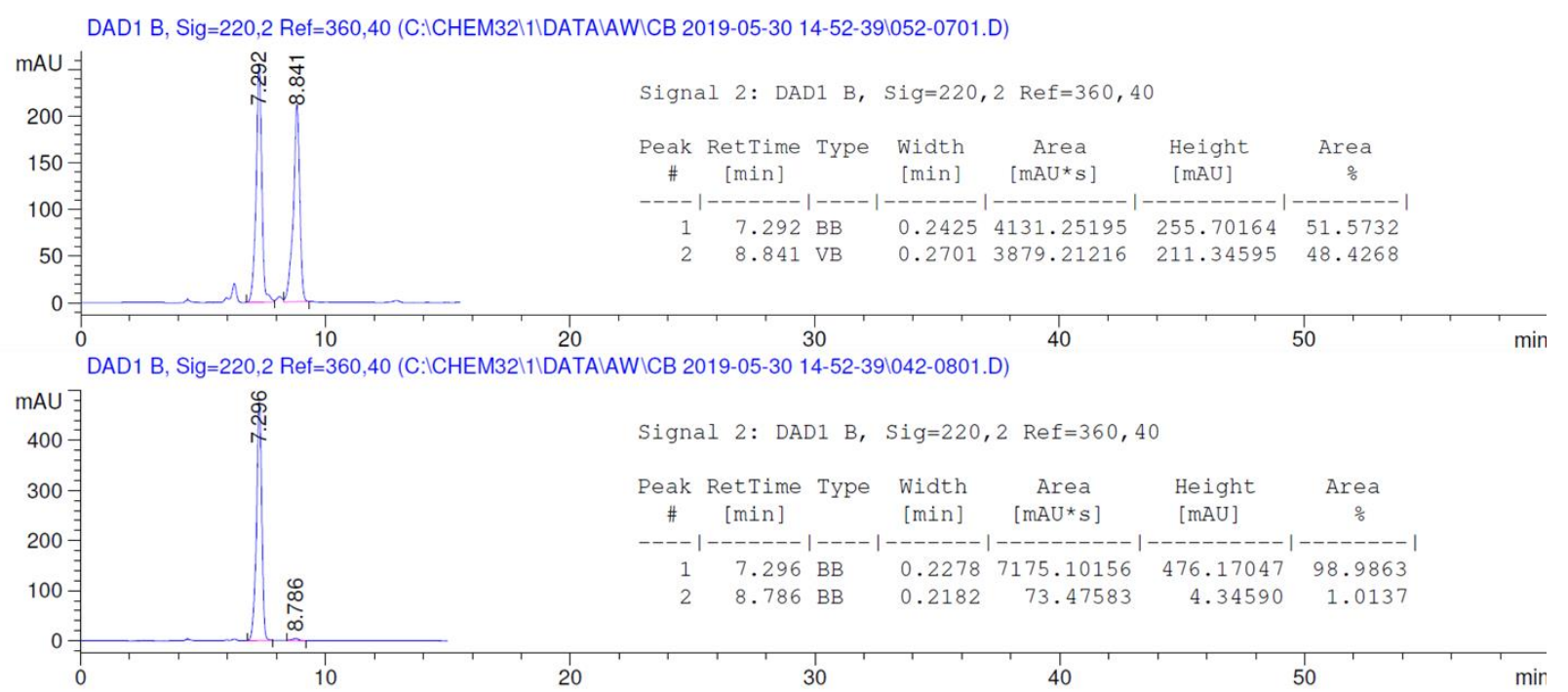
ethyl 3-((1S,2R)-1-hydroxy-2-phenyl-2-((4,4,5,5-tetramethyl-1,3,2-dioxaborolan-2yl)methyl)cyclobutyl)benzoate (2n)

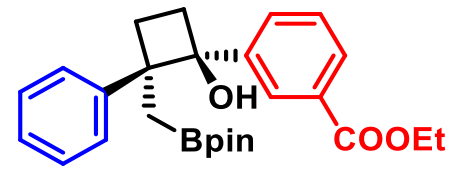

Prepared following General Procedure 3. Product was isolated by flash column chromatography $0 \% \rightarrow 5 \%$ acetone/pentane. Obtained $55 \mathrm{mg}$ of a white solid (63\% yield, 97.5:2.5 er).

${ }^{1} \mathbf{H}$ NMR $\left(500 \mathrm{MHz}, \mathrm{CDCl}_{3}\right) \delta 8.17(\mathrm{t}, J=1.8 \mathrm{~Hz}, 1 \mathrm{H}), 7.97(\mathrm{dt}, J=7.7,1.8 \mathrm{~Hz}, 1 \mathrm{H}), 7.69$ (ddd, $J=7.7,1.8,1.2 \mathrm{~Hz}, 1 \mathrm{H}), 7.44(\mathrm{t}, J=7.7 \mathrm{~Hz}, 1 \mathrm{H}), 7.40-7.30(\mathrm{~m}, 4 \mathrm{H}), 7.28-7.21(\mathrm{~m}, 1 \mathrm{H}), 4.46$ $-4.32(\mathrm{~m}, 2 \mathrm{H}), 3.04-2.89(\mathrm{~m}, 2 \mathrm{H}), 2.17-1.98(\mathrm{~m}, 3 \mathrm{H}), 1.41(\mathrm{t}, J=7.1 \mathrm{~Hz}, 3 \mathrm{H}), 0.97(\mathrm{~d}, J=$ $14.9 \mathrm{~Hz}, 1 \mathrm{H}), 0.90(\mathrm{~d}, J=14.9 \mathrm{~Hz}, 1 \mathrm{H}), 0.88(\mathrm{~s}, 6 \mathrm{H}), 0.86(\mathrm{~s}, 6 \mathrm{H})$.

${ }^{13} \mathrm{C}$ NMR $\left(126 \mathrm{MHz}, \mathrm{CDCl}_{3}\right) \delta 166.8,143.2,142.1,131.0,130.1,128.3,128.3,128.0,127.8$, 127.7, 126.7, 82.6, 81.9, 60.9, 54.1, 29.7, 26.1, 24.5, 24.3, 14.4.

IR (ATR) 3474, 2976, 1708, 1351, 1275, 1220, 1146, 1030, 969, 850, 761, 700

HRMS (ESI, M+NH 4 ) Calculated for $\mathrm{C}_{26} \mathrm{H}_{37} \mathrm{BNO}_{5} 453.2796$, found 453.2799

MP $115^{\circ} \mathrm{C}$

$\boldsymbol{\alpha}_{\boldsymbol{D}}^{\mathbf{2 0}}\left(\mathrm{c}=0.14, \mathrm{CH}_{2} \mathrm{Cl}_{2}\right) 11.4$

HPLC: IC, $10 \%$ IPA/Hex, $\mathrm{mL} / \mathrm{min}$

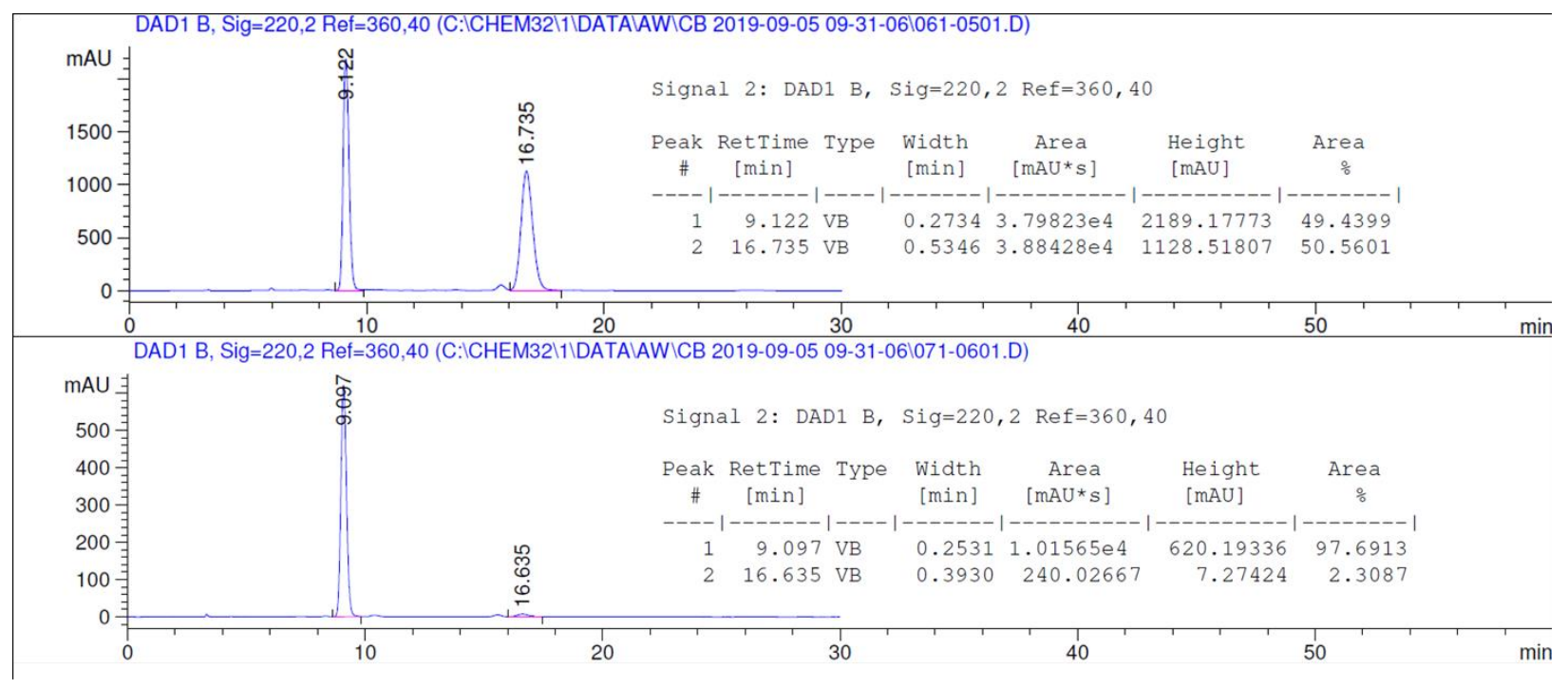


3-((1S,2R)-1-hydroxy-2-phenyl-2-((4,4,5,5-tetramethyl-1,3,2-dioxaborolan-2yl)methyl)cyclobutyl)benzonitrile (2o)

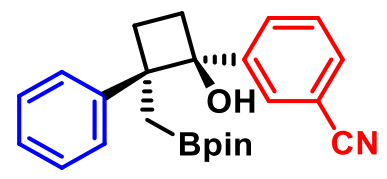

Prepared following General Procedure 3. Product was isolated by flash column chromatography 70 -> 90\% DCM/pentane. Obtained $20 \mathrm{mg}$ of a white solid (27\% yield, $97: 3 \mathrm{er}$ ).

${ }^{1} \mathbf{H}$ NMR $\left(500 \mathrm{MHz}, \mathrm{CDCl}_{3}\right) \delta 7.80(\mathrm{~s}, 1 \mathrm{H}), 7.75(\mathrm{~d}, J=7.9 \mathrm{~Hz}, 1 \mathrm{H}), 7.58(\mathrm{~d}, J=7.9 \mathrm{~Hz}, 1 \mathrm{H})$, $7.48(\mathrm{t}, J=7.9 \mathrm{~Hz}, 1 \mathrm{H}), 7.38(\mathrm{t}, J=7.6 \mathrm{~Hz}, 2 \mathrm{H}), 7.27(\mathrm{~d}, J=7.9 \mathrm{~Hz}, 3 \mathrm{H}), 3.02-2.81(\mathrm{~m}, 2 \mathrm{H})$, $2.16-1.96(\mathrm{~m}, 3 \mathrm{H}), 0.94(\mathrm{~d}, J=14.8 \mathrm{~Hz}, 1 \mathrm{H}), 0.89$ (s, 7H, overlap), 0.87 (s, 6H).

${ }^{13} \mathrm{C} \mathrm{NMR}\left(126 \mathrm{MHz}, \mathrm{CDCl}_{3}\right) \delta 144.4,141.5,131.1,130.7,130.4,128.7,128.5,127.7,127.0$, $119.1,112.1,82.8,81.3,54.3,30.0,26.1,24.5,24.3,1.0$.

IR (ATR) 3496, 2940, 2225, 1357, 1327, 1262, 1146, 1058, 975, 890, 792, 682, 647

HRMS (ESI, M+NH 4 ) Calculated for $\mathrm{C}_{24} \mathrm{H}_{32} \mathrm{BN}_{2} \mathrm{O}_{3} 406.2537$, found 406.2544

MP $117^{\circ} \mathrm{C}$

$\boldsymbol{\alpha}_{\boldsymbol{D}}^{\mathbf{2 0}}\left(\mathrm{c}=0.14, \mathrm{CH}_{2} \mathrm{Cl}_{2}\right) 20.0$

HPLC: IC, 10\% IPA/Hex, mL/min

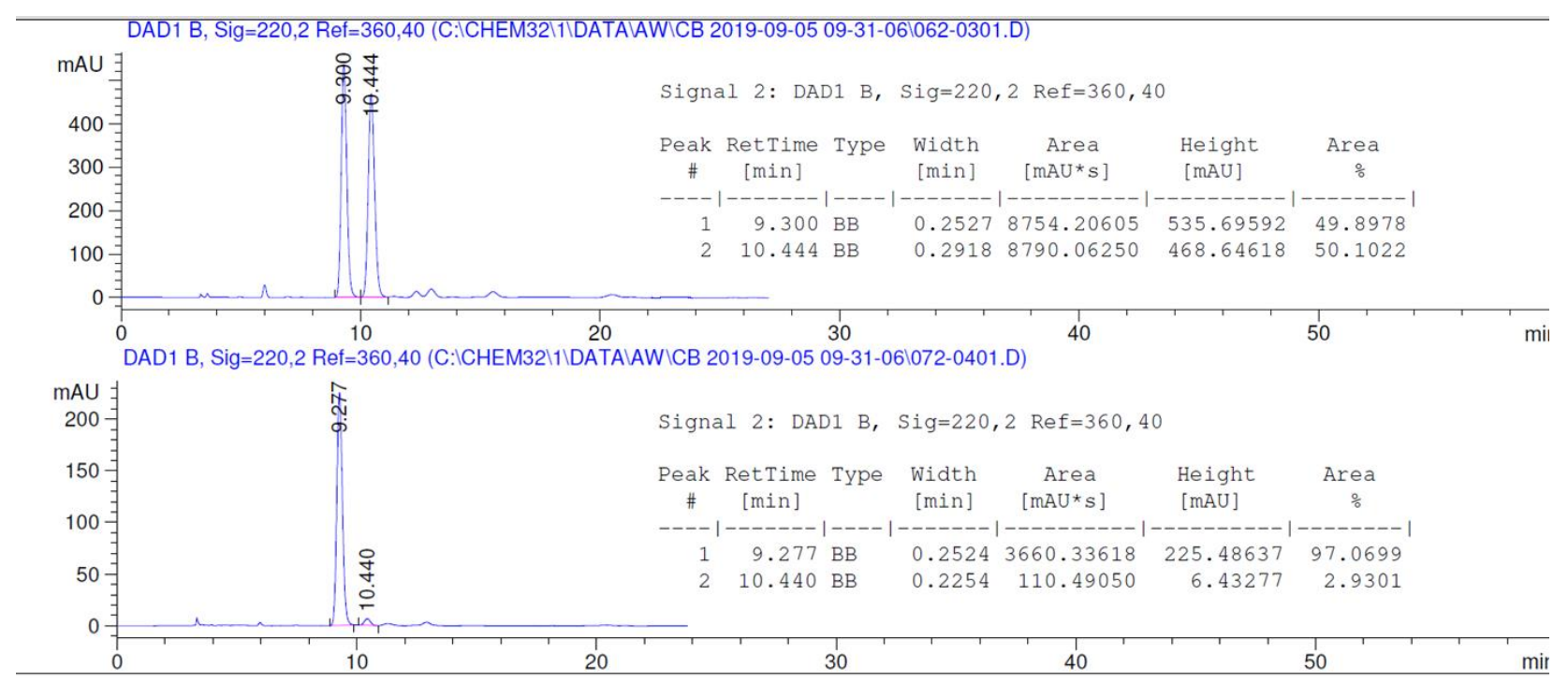


(1R,2R)-2-phenyl-2-((4,4,5,5-tetramethyl-1,3,2-dioxaborolan-2-yl)methyl)-1-(otolyl)cyclobutan-1-ol (2p)

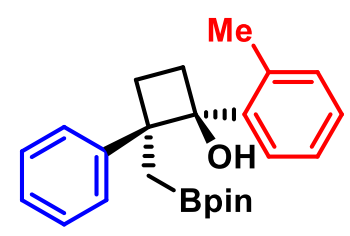

Prepared following General Procedure 3. Product was isolated by flash column chromatography 0 -> 5\% acetone/pentane. Obtained $62 \mathrm{mg}$ of a white solid (82\% yield, 97:3 er).

${ }^{1} \mathbf{H}$ NMR $\left(500 \mathrm{MHz}, \mathrm{CDCl}_{3}\right) \delta 7.68-7.60(\mathrm{~m}, 2 \mathrm{H}), 7.47-7.35(\mathrm{~m}, 3 \mathrm{H}), 7.29-7.26(\mathrm{~m}, 1 \mathrm{H})$, $7.22-7.11(\mathrm{~m}, 3 \mathrm{H}), 3.01(\mathrm{ddd}, J=12.8,8.6,5.0 \mathrm{~Hz}, 1 \mathrm{H}), 2.81$ (ddd, $J=12.1,9.5,5.0 \mathrm{~Hz}, 1 \mathrm{H})$, $2.43(\mathrm{~s}, 3 \mathrm{H}), 2.15(\mathrm{dt}, J=12.4,8.8 \mathrm{~Hz}, 1 \mathrm{H}), 1.98(\mathrm{dt}, J=12.0,8.4 \mathrm{~Hz}, 1 \mathrm{H}), 1.80(\mathrm{~s}, 1 \mathrm{H}), 1.47(\mathrm{~d}$, $J=15.3 \mathrm{~Hz}, 1 \mathrm{H}), 0.96(\mathrm{~s}, 6 \mathrm{H}), 0.89(\mathrm{~s}, 6 \mathrm{H}), 0.79$ (d, $J=15.3 \mathrm{~Hz}, 1 \mathrm{H})$.

${ }^{13} \mathrm{C}$ NMR $\left(126 \mathrm{MHz}, \mathrm{CDCl}_{3}\right) \delta 142.0,139.8,137.7,132.3,128.6,128.2,127.2,127.0,126.6$, $125.4,83.7,82.6,55.1,33.4,27.1,24.6,24.2,22.6$.

IR (ATR) 3524, 2977, 1444, 1346, 1320, 1141, 1108, 967, 883, 845, 771, 736, 696, 593

HRMS (DART, M- $\mathrm{H}_{2} \mathrm{O}+\mathrm{H}$ ) Calculated for $\mathrm{C}_{24} \mathrm{H}_{30} \mathrm{~B}_{1} \mathrm{O}_{2} 361.2334$, found 361.2339

MP $61{ }^{\circ} \mathrm{C}$

$\boldsymbol{\alpha}_{\boldsymbol{D}}^{\mathbf{2 0}}\left(\mathrm{c}=0.260, \mathrm{CH}_{2} \mathrm{Cl}_{2}\right)-26.7$

HPLC: IA, $4 \%$ IPA/Hex, $0.75 \mathrm{~mL} / \mathrm{min}$
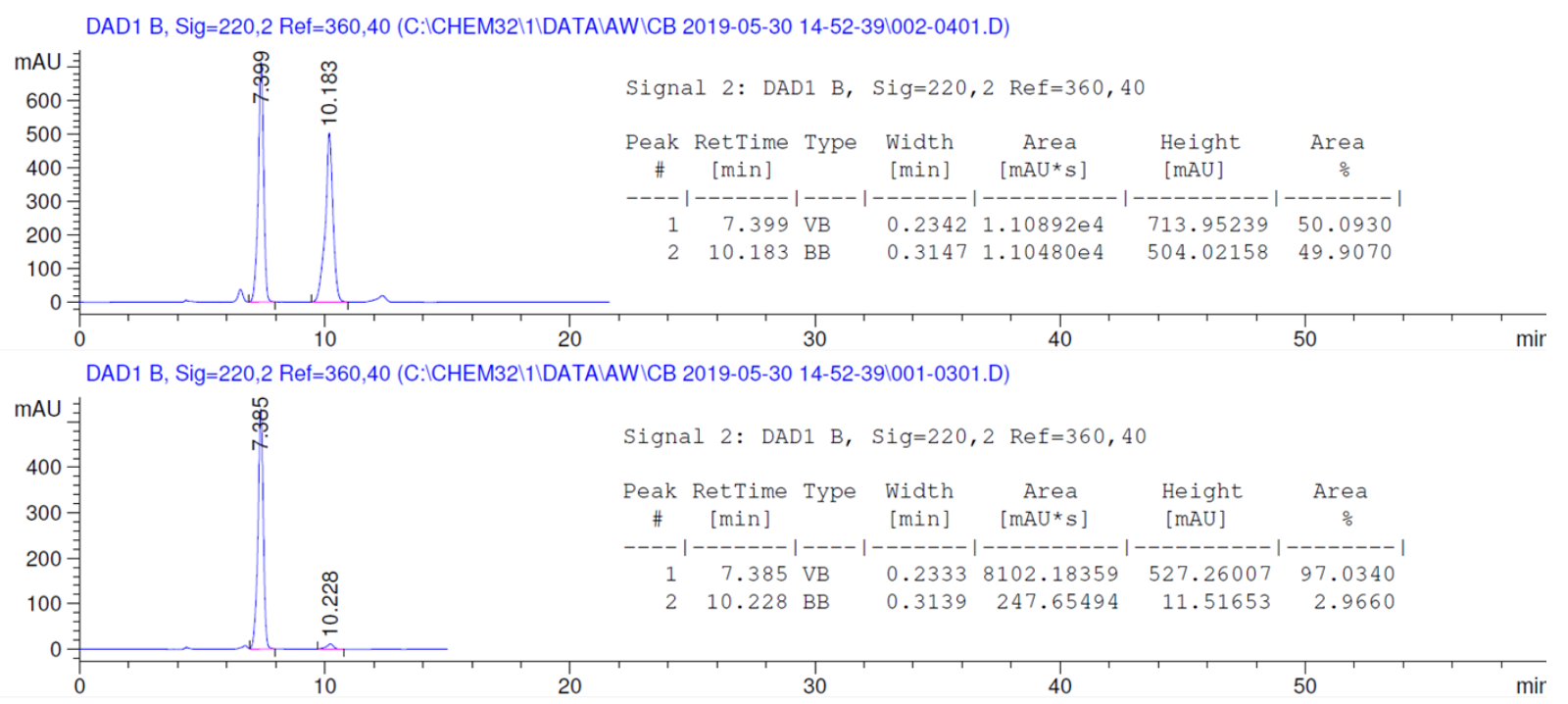
(1R,2R)-2-phenyl-2-((4,4,5,5-tetramethyl-1,3,2-dioxaborolan-2-yl)methyl)-1-(thiophen-2yl)cyclobutan-1-ol (2q)

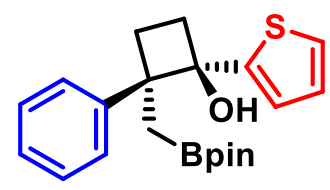

Prepared following General Procedure 3. Product was isolated by flash column chromatography $0->5 \%$ acetone/pentane. Obtained $67 \mathrm{mg}$ of a white solid (90\% yield, 96:4 er).

${ }^{1} \mathbf{H}$ NMR $\left(500 \mathrm{MHz}, \mathrm{CDCl}_{3}\right) \delta 7.39-7.31(\mathrm{~m}, 4 \mathrm{H}), 7.27(\mathrm{dd}, J=5.0,1.2 \mathrm{~Hz}, 1 \mathrm{H}), 7.26-7.19$ $(\mathrm{m}, 1 \mathrm{H}), 7.05(\mathrm{dd}, J=3.6,1.2 \mathrm{~Hz}, 1 \mathrm{H}), 7.02(\mathrm{dd}, J=5.0,3.6 \mathrm{~Hz}, 1 \mathrm{H}), 2.96-2.85(\mathrm{~m}, 1 \mathrm{H}), 2.85$ $-2.75(\mathrm{~m}, 1 \mathrm{H}), 2.22-2.12(\mathrm{~m}, 3 \mathrm{H}), 1.23(\mathrm{~d}, J=14.9 \mathrm{~Hz}, 1 \mathrm{H}), 1.13(\mathrm{dd}, J=14.9,1.7 \mathrm{~Hz}, 1 \mathrm{H})$, $0.92(\mathrm{~s}, 6 \mathrm{H}), 0.91(\mathrm{~s}, 6 \mathrm{H})$.

${ }^{13} \mathrm{C}$ NMR $\left(126 \mathrm{MHz}, \mathrm{CDCl}_{3}\right) \delta 148.6,142.6,128.3,127.5,126.7,126.7,124.7,124.5,82.6$, $81.2,54.3,32.2,26.2,24.6,24.4$.

IR (ATR) 3440, 2975, 2946, 1360, 1337, 1291, 1214, 1135, 969, 841, 719, 695, 597

HRMS (DART, M-H $\mathrm{H}_{2} \mathrm{O}+\mathrm{H}$ ) Calculated for $\mathrm{C}_{21} \mathrm{H}_{26} \mathrm{~B}_{1} \mathrm{O}_{2} \mathrm{~S}_{1} 353.1747$, found 353.1752

MP $119^{\circ} \mathrm{C}$

$\boldsymbol{\alpha}_{\boldsymbol{D}}^{\mathbf{2 0}}\left(\mathrm{c}=0.23, \mathrm{CH}_{2} \mathrm{Cl}_{2}\right)-29.8$

HPLC: IA, $4 \%$ IPA/Hex, $0.75 \mathrm{~mL} / \mathrm{min}$

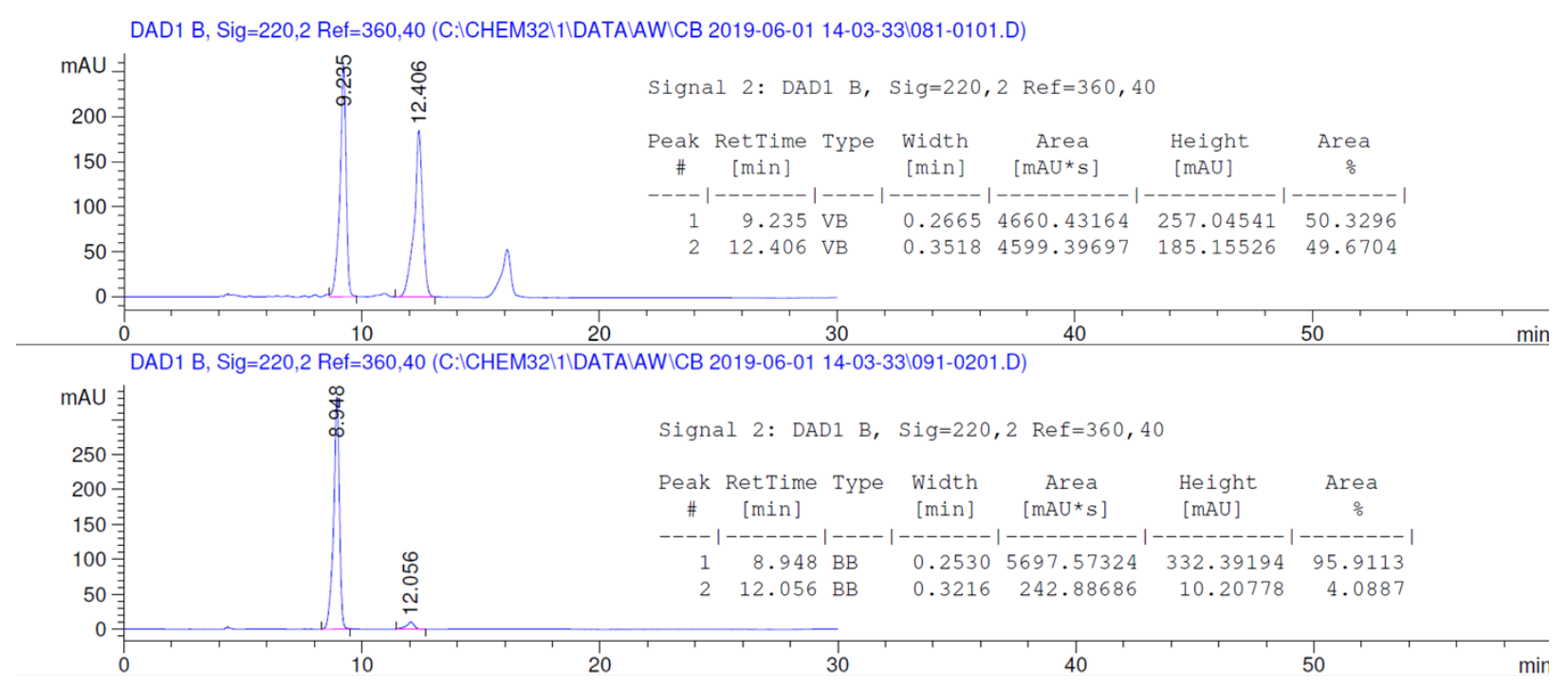


(1S,2R)-1-(1-methyl-1H-indol-5-yl)-2-phenyl-2-((4,4,5,5-tetramethyl-1,3,2-dioxaborolan-2yl)methyl)cyclobutan-1-ol (2r)<smiles>Cn1ccc2cc([C@]3(O)CC[C@@]3(Cc3ccccc3)c3ccccc3)ccc21</smiles>

Prepared following General Procedure 3. Product was isolated by flash column chromatography $0->5 \%$ acetone/pentane. Obtained $69 \mathrm{mg}$ of a white solid (83\% yield, 95:5 er).

${ }^{1} \mathbf{H}$ NMR $\left(500 \mathrm{MHz}, \mathrm{CDCl}_{3}\right) \delta 7.75(\mathrm{dd}, J=1.8,0.7 \mathrm{~Hz}, 1 \mathrm{H}), 7.45-7.42(\mathrm{~m}, 2 \mathrm{H}), 7.41-7.36$ $(\mathrm{m}, 3 \mathrm{H}), 7.32(\mathrm{dt}, J=8.6,0.8 \mathrm{~Hz}, 1 \mathrm{H}), 7.27-7.23(\mathrm{~m}, 1 \mathrm{H}), 7.06(\mathrm{~d}, J=3.0 \mathrm{~Hz}, 1 \mathrm{H}), 6.51(\mathrm{dd}, J$ $=3.1,0.8 \mathrm{~Hz}, 1 \mathrm{H}), 3.80(\mathrm{~s}, 3 \mathrm{H}), 3.12-3.05(\mathrm{~m}, 1 \mathrm{H}), 3.00(\mathrm{ddd}, J=12.5,9.4,7.7 \mathrm{~Hz}, 1 \mathrm{H}), 2.15$ $-2.05(\mathrm{~m}, 2 \mathrm{H}), 2.02(\mathrm{~s}, 1 \mathrm{H}), 1.01(\mathrm{~s}, 2 \mathrm{H}), 0.90(\mathrm{~s}, 6 \mathrm{H}), 0.87(\mathrm{~s}, 6 \mathrm{H})$.

${ }^{13} \mathrm{C}$ NMR $\left(126 \mathrm{MHz}, \mathrm{CDCl}_{3}\right) \delta 143.1,136.0,133.9,129.0,128.1,128.1,127.9,126.3,120.6$, $118.8,118.8,108.6,101.2,101.2,82.9,82.4,54.1,32.9,30.0,26.2,24.6,24.3$.

IR (ATR) 3586, 2983, 1499, 1363, 1327, 1167, 1142, 963, 888, 803, 739, 696

HRMS (DART, M-H $\mathrm{H}_{2} \mathrm{O}+\mathrm{H}$ ) Calculated for $\mathrm{C}_{26} \mathrm{H}_{31} \mathrm{~B}_{1} \mathrm{~N}_{1} \mathrm{O}_{2} 400.2448$, found 400.2449

MP $177^{\circ} \mathrm{C}$

$\boldsymbol{\alpha}_{D}^{\mathbf{2 0}}\left(\mathrm{c}=0.175, \mathrm{CH}_{2} \mathrm{Cl}_{2}\right) 58.7$

HPLC: IA, $6 \%$ IPA/hex, $1 \mathrm{~mL} / \mathrm{min}$

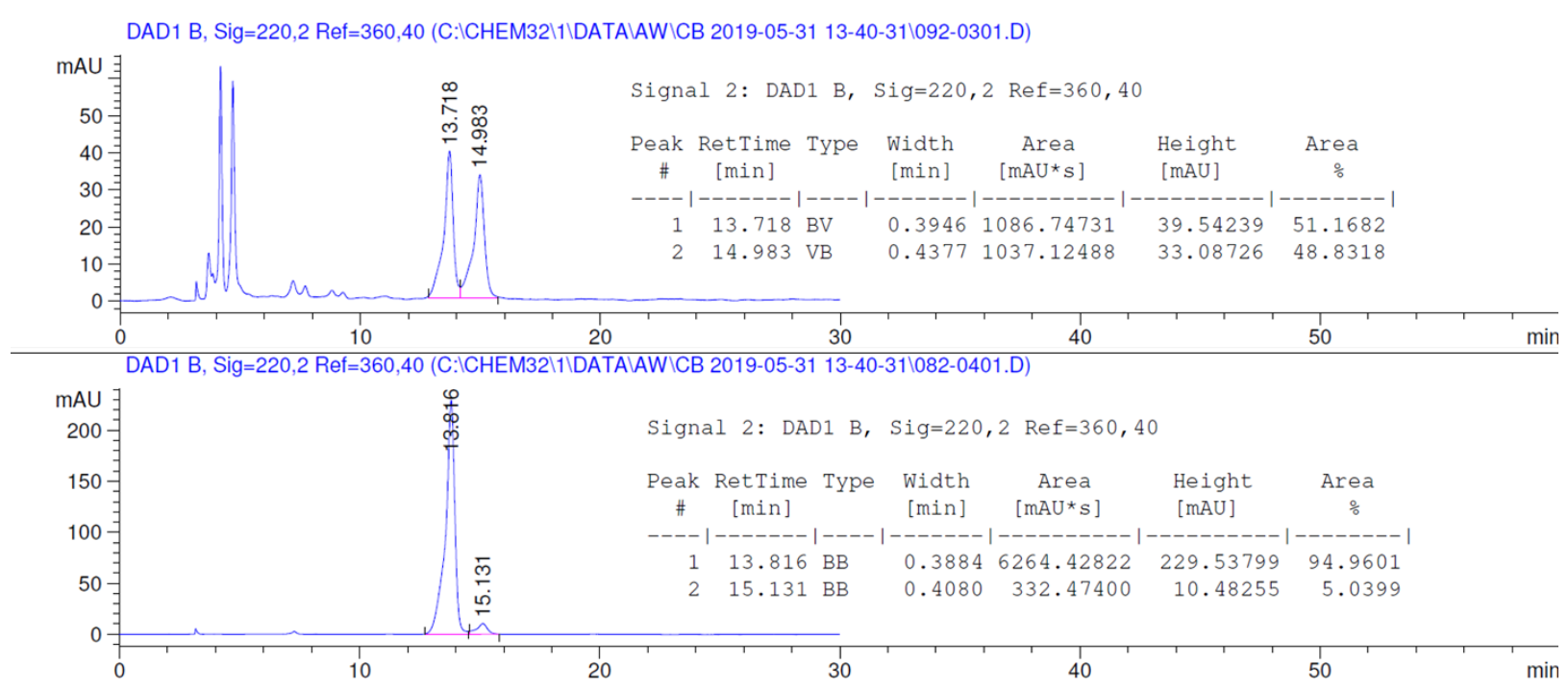


(1S,2R)-1-phenyl-2-((4,4,5,5-tetramethyl-1,3,2-dioxaborolan-2-yl)methyl)-2-(ptolyl)cyclobutan-1-ol (2s)<smiles>Cc1ccc([C@]2(CBr)CC[C@@]2(O)c2ccccc2)cc1</smiles>

Prepared following General Procedure 3. Product was isolated by flash column chromatography $0->5 \%$ acetone/pentane. Obtained $55 \mathrm{mg}$ of a white solid (73\% yield, 97:3 er).

${ }^{1} \mathbf{H}$ NMR $\left(500 \mathrm{MHz}, \mathrm{CDCl}_{3}\right) \delta 7.48(\mathrm{dd}, J=8.4,1.2 \mathrm{~Hz}, 2 \mathrm{H}), 7.37(\mathrm{t}, J=7.7 \mathrm{~Hz}, 2 \mathrm{H}), 7.29-$ $7.25(\mathrm{~m}, 1 \mathrm{H}), 7.25-7.21(\mathrm{~m}, 2 \mathrm{H}), 7.17(\mathrm{~d}, J=7.7 \mathrm{~Hz}, 2 \mathrm{H}), 2.98-2.89(\mathrm{~m}, 2 \mathrm{H}), 2.34(\mathrm{~s}, 3 \mathrm{H})$, $2.11-1.95(\mathrm{~m}, 3 \mathrm{H}), 0.99(\mathrm{~d}, J=14.9 \mathrm{~Hz}, 1 \mathrm{H}), 0.94(\mathrm{~d}, J=14.9 \mathrm{~Hz}, 1 \mathrm{H}), 0.90$ (s, 6H), 0.89 (s, $6 \mathrm{H})$.

${ }^{13}$ C NMR (126 MHz, $\left.\mathrm{CDCl}_{3}\right) \delta 142.8,139.3,136.0,128.9,127.9,127.7,127.0,126.5,82.5$, 82.0, 53.7, 29.5, 26.2, 24.6, 24.3, 21.0.

IR (ATR) 3562, 2978, 2922, 1446, 1353, 1330, 1208, 1138, 970, 939, 892, 845, 807, 744, 703, 660,576

HRMS (DART, M+H) Calculated for $\mathrm{C}_{24} \mathrm{H}_{35} \mathrm{~B}_{1} \mathrm{~N}_{1} \mathrm{O}_{3} 396.2710$, found 396.2719

MP $53{ }^{\circ} \mathrm{C}$

$\boldsymbol{\alpha}_{D}^{\mathbf{2 0}}\left(\mathrm{c}=0.140, \mathrm{CH}_{2} \mathrm{Cl}_{2}\right) 28.6$

HPLC: IC, $4 \%$ IPA/Hex, $0.75 \mathrm{~mL} / \mathrm{min}$

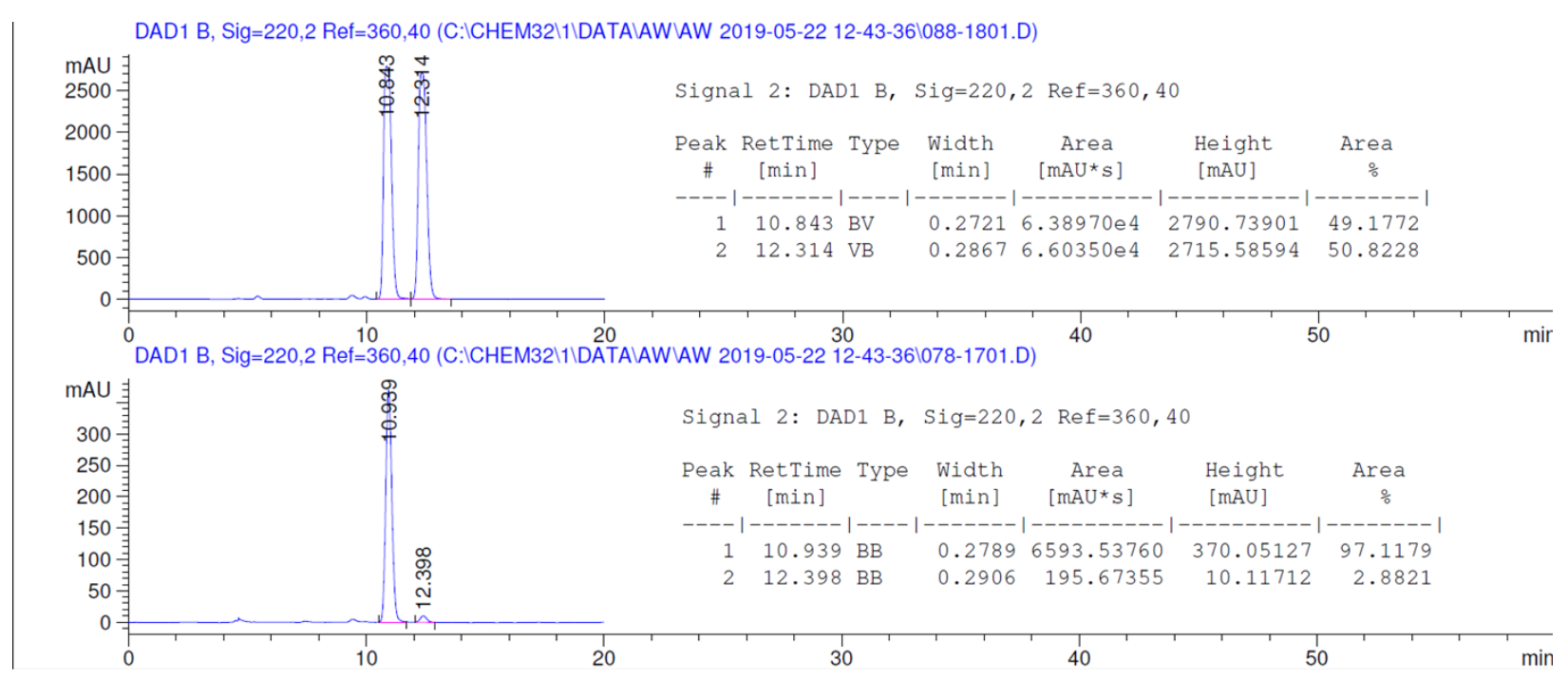


(1S,2R)-1-(4-fluorophenyl)-2-((4,4,5,5-tetramethyl-1,3,2-dioxaborolan-2-yl)methyl)-2-(ptolyl)cyclobutan-1-ol (2t)<smiles>Cc1ccc([C@]2(CC[Pb])CC[C@@]2(O)c2ccc(F)cc2)cc1</smiles>

Prepared following General Procedure 3. Product was isolated by flash column chromatography 0 -> 5\% acetone/pentane. Obtained $48 \mathrm{mg}$ of a white solid (61\% yield, 97.5:2.5 er).

${ }^{1} \mathbf{H}$ NMR $\left(500 \mathrm{MHz}, \mathrm{CDCl}_{3}\right) \delta 7.44(\mathrm{dd}, J=8.8,5.4 \mathrm{~Hz}, 2 \mathrm{H}), 7.20(\mathrm{~d}, J=8.1 \mathrm{~Hz}, 2 \mathrm{H}), 7.16(\mathrm{~d}, J$ $=8.1 \mathrm{~Hz}, 2 \mathrm{H}), 7.04(\mathrm{t}, J=8.8 \mathrm{~Hz}, 2 \mathrm{H}), 2.95-2.84(\mathrm{~m}, 2 \mathrm{H}), 2.33(\mathrm{~s}, 3 \mathrm{H}), 2.08-1.98(\mathrm{~m}, 3 \mathrm{H})$, $0.93(\mathrm{~s}, 2 \mathrm{H}), 0.90(\mathrm{~s}, 6 \mathrm{H}), 0.88(\mathrm{~s}, 6 \mathrm{H})$.

${ }^{13} \mathrm{C}$ NMR $\left(126 \mathrm{MHz}, \mathrm{CDCl}_{3}\right) \delta 162.0(\mathrm{~d}, J=245.4 \mathrm{~Hz}), 138.9,138.7(\mathrm{~d}, J=3.1 \mathrm{~Hz}), 136.2$, $129.0,128.2(\mathrm{~d}, J=8.0 \mathrm{~Hz}), 127.6,114.6(\mathrm{~d}, J=21.1 \mathrm{~Hz}), 82.6,81.6,53.8(\mathrm{~d}, J=0.9 \mathrm{~Hz}), 29.9$, 26.1, 24.6, 24.3, 21.0.

${ }^{19}$ F NMR $\left(377 \mathrm{MHz}, \mathrm{CDCl}_{3}\right) \delta-116.3$.

IR (ATR) 3558, 2979, 2919, 1598, 1508, 1352, 1333, 1219, 1135, 954, 827, 761, 748, 599, 575

HRMS (DART, M+NH 4 ) Calculated for $\mathrm{C}_{24} \mathrm{H}_{34} \mathrm{~B}_{1} \mathrm{~F}_{1} \mathrm{~N}_{1} \mathrm{O}_{3} 414.2616$, found 414.2618

MP $86{ }^{\circ} \mathrm{C}$

$\boldsymbol{\alpha}_{\boldsymbol{D}}^{\mathbf{2 0}}\left(\mathrm{c}=0.225, \mathrm{CH}_{2} \mathrm{Cl}_{2}\right) 28.1$

HPLC: IA, 4\% IPA/Hex, $0.75 \mathrm{~mL} / \mathrm{min}$

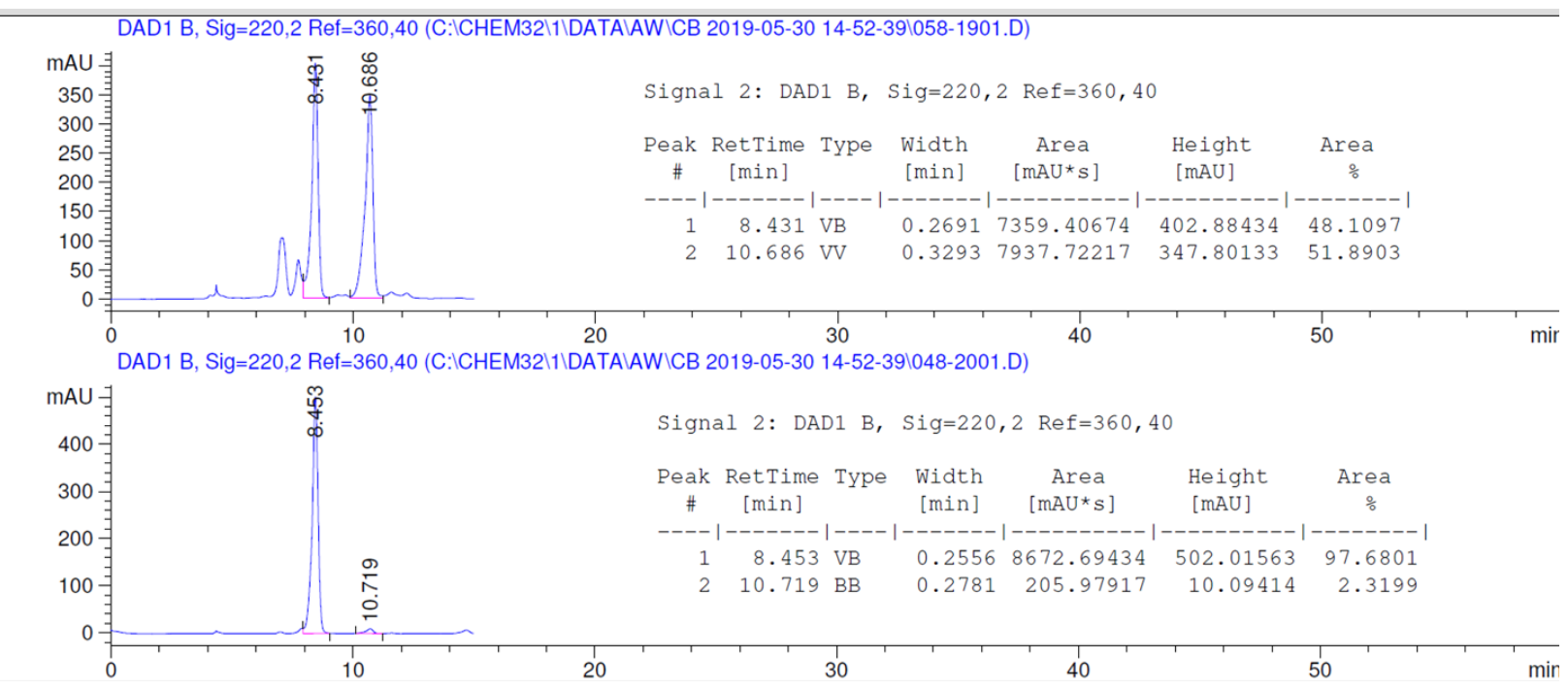


(1S,2R)-2-(4-fluorophenyl)-1-phenyl-2-((4,4,5,5-tetramethyl-1,3,2-dioxaborolan-2yl)methyl)cyclobutan-1-ol (2u)<smiles>[B]C[C@@]1(c2ccc(F)cc2)CC[C@@]1(O)c1ccccc1</smiles>

Prepared following General Procedure 3. Product was isolated by flash column chromatography $0->5 \%$ acetone/pentane. Obtained $48 \mathrm{mg}$ of a white solid (63\% yield, 99:1 er).

${ }^{1} \mathbf{H}$ NMR $\left(500 \mathrm{MHz}, \mathrm{CDCl}_{3}\right) \delta 7.49-7.43(\mathrm{~m}, 2 \mathrm{H}), 7.37(\mathrm{dd}, J=8.4,6.9 \mathrm{~Hz}, 2 \mathrm{H}), 7.33-7.26$ $(\mathrm{m}, 3 \mathrm{H}), 7.05(\mathrm{t}, J=8.4 \mathrm{~Hz}, 2 \mathrm{H}), 3.02-2.87(\mathrm{~m}, 2 \mathrm{H}), 2.14-2.06(\mathrm{~m}, 1 \mathrm{H}), 2.03-1.94(\mathrm{~m}, 1 \mathrm{H})$, $1.90(\mathrm{~s}, 1 \mathrm{H}), 1.03(\mathrm{~d}, J=15.1 \mathrm{~Hz}, 1 \mathrm{H}), 0.92(\mathrm{~s}, 6 \mathrm{H}), 0.90(\mathrm{~s}, 6 \mathrm{H}), 0.89$ (d, $J=15.1 \mathrm{~Hz}, 1 \mathrm{H})$.

${ }^{13} \mathrm{C} \mathrm{NMR}\left(126 \mathrm{MHz}, \mathrm{CDCl}_{3}\right) \delta 161.6(\mathrm{~d}, J=245.0 \mathrm{~Hz}), 142.5,138.7(\mathrm{~d}, J=3.2 \mathrm{~Hz}), 129.3(\mathrm{~d}, J$ $=7.8 \mathrm{~Hz}), 128.0,127.2,126.4,114.9(\mathrm{~d}, J=20.9 \mathrm{~Hz}), 82.7,82.3(\mathrm{~d}, J=0.8 \mathrm{~Hz}), 53.3,29.3,26.4$, 24.6, 24.3.

${ }^{19}$ F NMR $\left(377 \mathrm{MHz}, \mathrm{CDCl}_{3}\right) \delta-116.8$.

IR (ATR) 3456, 2974, 2934, 2181, 1509, 1359, 1338, 1289, 1215, 1136, 950, 846, 828, 705, 589, 578

HRMS (ESI, M+Na) Calculated for $\mathrm{C}_{23} \mathrm{H}_{28} \mathrm{~B}_{1} \mathrm{~F}_{1} \mathrm{Na}_{1} \mathrm{O}_{3} 404.2044$, found 404.2037

MP $88^{\circ} \mathrm{C}$

$\boldsymbol{\alpha}_{\boldsymbol{D}}^{\mathbf{2 0}}\left(\mathrm{c}=0.17, \mathrm{CH}_{2} \mathrm{Cl}_{2}\right) 4.7$

HPLC: IC, $4 \%$ IPA/Hex, $0.75 \mathrm{~mL} / \mathrm{min}$

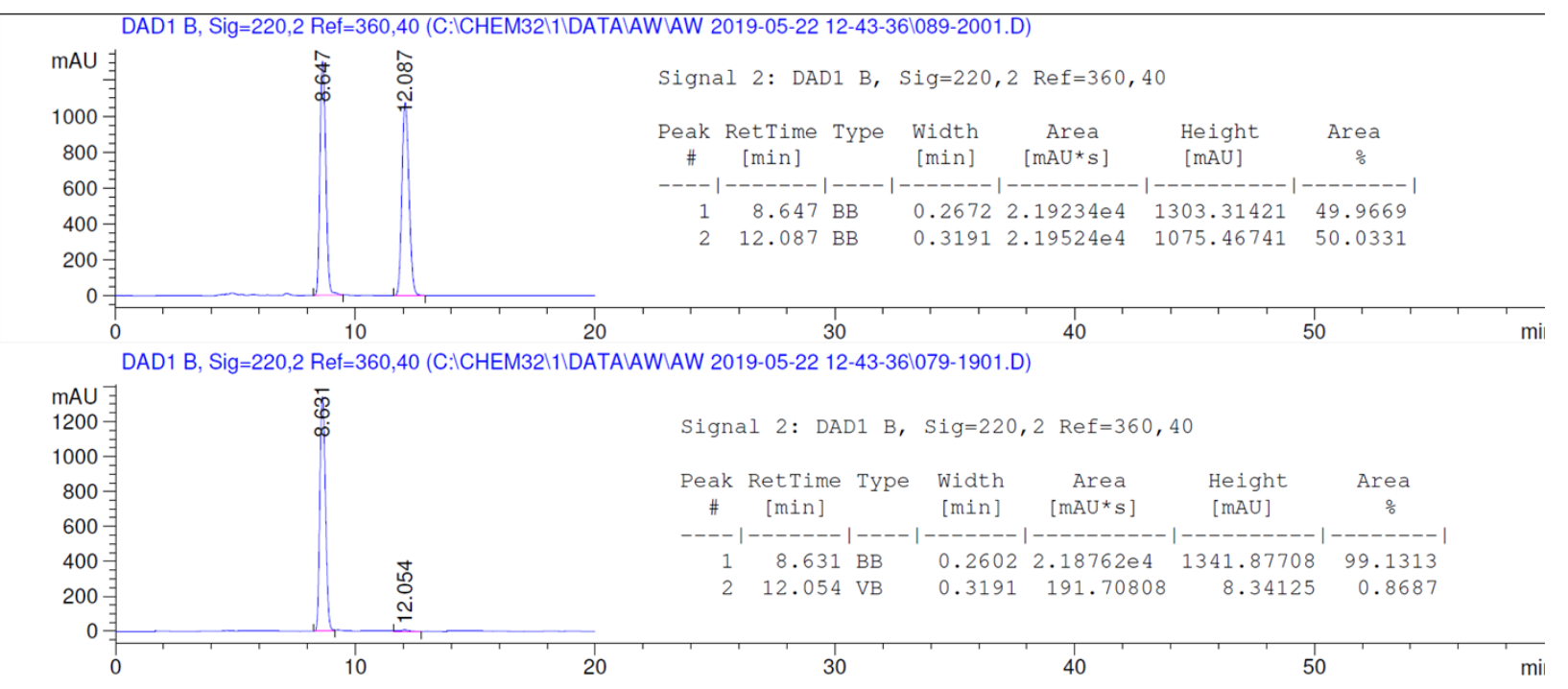


(1S,2R)-2-(3-fluoro-4-methoxyphenyl)-1-phenyl-2-((4,4,5,5-tetramethyl-1,3,2-dioxaborolan2-yl)methyl)cyclobutan-1-ol (2v)<smiles>COc1ccc([C@]2(CBr)CC[C@@]2(O)c2ccccc2)cc1F</smiles>

Prepared following General Procedure 3. Product was isolated by flash column chromatography $0->5 \%$ acetone/pentane. Obtained $56 \mathrm{mg}$ of a white solid (68\% yield, 98.5:1.5 er).

${ }^{1} \mathbf{H}$ NMR $\left(500 \mathrm{MHz}, \mathrm{CDCl}_{3}\right) \delta 7.48-7.43(\mathrm{~m}, 2 \mathrm{H}), 7.40-7.33(\mathrm{~m}, 2 \mathrm{H}), 7.30-7.26(\mathrm{~m}, 1 \mathrm{H})$, $7.10(\mathrm{dd}, J=13.1,2.2 \mathrm{~Hz}, 1 \mathrm{H}), 7.03(\mathrm{ddd}, J=8.5,2.2,1.0 \mathrm{~Hz}, 1 \mathrm{H}), 6.96(\mathrm{t}, J=8.6 \mathrm{~Hz}, 1 \mathrm{H}), 3.88$ (s, 3H), $2.94(\mathrm{dt}, J=11.9,8.3 \mathrm{~Hz}, 1 \mathrm{H}), 2.90-2.78(\mathrm{~m}, 1 \mathrm{H}), 2.08$ (ddd, $J=11.9,8.6,4.4 \mathrm{~Hz}$, $1 \mathrm{H}), 2.02-1.95(\mathrm{~m}, 2 \mathrm{H}), 1.00(\mathrm{~d}, J=15.1 \mathrm{~Hz}, 1 \mathrm{H}), 0.94$ (s, 6H), 0.91 (s, 6H), 0.85 (dd, $J=15.1$, $1.4 \mathrm{~Hz}, 1 \mathrm{H})$.

${ }^{13}$ C NMR $\left(126 \mathrm{MHz}, \mathrm{CDCl}_{3}\right) \delta 152.2(\mathrm{~d}, J=245.3 \mathrm{~Hz}), 146.0(\mathrm{~d}, J=10.8 \mathrm{~Hz}), 142.5,136.3(\mathrm{~d}$, $J=5.4 \mathrm{~Hz}), 128.0,127.2,126.4,123.4(\mathrm{~d}, J=3.4 \mathrm{~Hz}), 115.8(\mathrm{~d}, J=18.6 \mathrm{~Hz}), 113.1(\mathrm{~d}, J=2.1$ $\mathrm{Hz}), 82.7,82.2,56.3(\mathrm{~d}, J=1.5 \mathrm{~Hz}), 53.2(\mathrm{~d}, J=1.1 \mathrm{~Hz}), 29.3,26.5,24.6,24.4$.

${ }^{19}$ F NMR $\left(377 \mathrm{MHz}, \mathrm{CDCl}_{3}\right) \delta-135.4$.

IR (ATR) 3568, 2981, 2927, 1514, 1421, 1357, 1338, 1302, 1272, 1136, 1113, 1027, 973, 899, $820,759,705,628$

HRMS (DART, M-H $\mathrm{H}_{2} \mathrm{O}+\mathrm{H}$ ) Calculated for $\mathrm{C}_{24} \mathrm{H}_{29} \mathrm{~B}_{1} \mathrm{~F}_{1} \mathrm{O}_{3} 395.2194$, found 395.2195

MP $142{ }^{\circ} \mathrm{C}$

$\boldsymbol{\alpha}_{D}^{\mathbf{2 0}}\left(\mathrm{c}=0.235, \mathrm{CH}_{2} \mathrm{Cl}_{2}\right) 40.0$

HPLC: IA, 4\% IPA/Hex, $0.75 \mathrm{~mL} / \mathrm{min}$
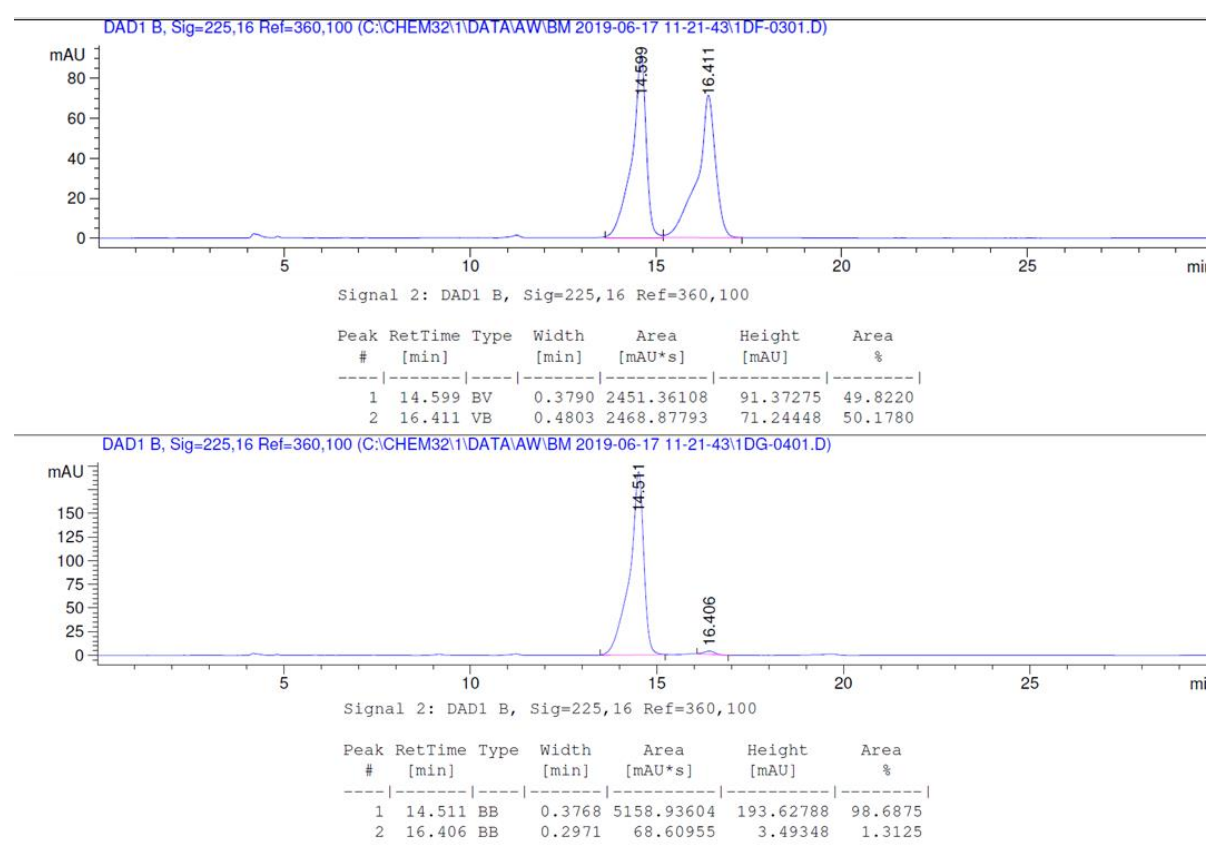
(1S,2R)-1-phenyl-2-((4,4,5,5-tetramethyl-1,3,2-dioxaborolan-2-yl)methyl)-2-(mtolyl)cyclobutan-1-ol (2w)<smiles>Cc1cccc([C@]2(CBr)CC[C@@]2(O)c2ccccc2)c1</smiles>

Prepared following General Procedure 3. Product was isolated by flash column chromatography 0 -> 5\% acetone/pentane. Obtained $66 \mathrm{mg}$ of a white solid (87\% yield, 96.5:3.5 er).

${ }^{1} \mathbf{H}$ NMR $\left(500 \mathrm{MHz}, \mathrm{CDCl}_{3}\right) \delta 7.51-7.48(\mathrm{~m}, 2 \mathrm{H}), 7.39-7.35(\mathrm{~m}, 2 \mathrm{H}), 7.29-7.25(\mathrm{~m}, 1 \mathrm{H})$, $7.24(\mathrm{t}, J=7.6 \mathrm{~Hz}, 1 \mathrm{H}), 7.15(\mathrm{~s}, 1 \mathrm{H}), 7.10(\mathrm{~d}, J=7.6 \mathrm{~Hz}, 1 \mathrm{H}), 7.04(\mathrm{~d}, J=7.6 \mathrm{~Hz}, 1 \mathrm{H}), 2.98-$ $2.90(\mathrm{~m}, 2 \mathrm{H}), 2.36(\mathrm{~s}, 3 \mathrm{H}), 2.12-1.98(\mathrm{~m}, 3 \mathrm{H}), 1.01(\mathrm{~d}, J=15.2 \mathrm{~Hz}, 1 \mathrm{H}), 0.93(\mathrm{~d}, J=15.2 \mathrm{~Hz}$, $1 \mathrm{H}), 0.90(\mathrm{~s}, 6 \mathrm{H}), 0.88(\mathrm{~s}, 6 \mathrm{H})$.

${ }^{13} \mathrm{C}$ NMR $\left(126 \mathrm{MHz}, \mathrm{CDCl}_{3}\right) \delta 142.8,142.5,137.6,128.6,128.2,127.8,127.2,127.0,126.5$, $124.7,82.5,82.2,53.9,29.5,26.3,24.6,24.3,21.6$.

IR (ATR) 3531, 2977, 2147, 2014, 1447, 1343, 1326, 1206, 1137, 1070, 968, 826, 792, 769, 608

HRMS (DART, M+NH4) Calculated for $\mathrm{C}_{24} \mathrm{H}_{35} \mathrm{~B}_{1} \mathrm{~N}_{1} \mathrm{O}_{3} 396.2710$, found 396.2704

MP $86{ }^{\circ} \mathrm{C}$

$\boldsymbol{\alpha}_{\boldsymbol{D}}^{\mathbf{2 0}}\left(\mathrm{c}=0.33, \mathrm{CH}_{2} \mathrm{Cl}_{2}\right) 2.5$

HPLC: IC, $4 \%$ IPA/Hex, $0.75 \mathrm{~mL} / \mathrm{min}$

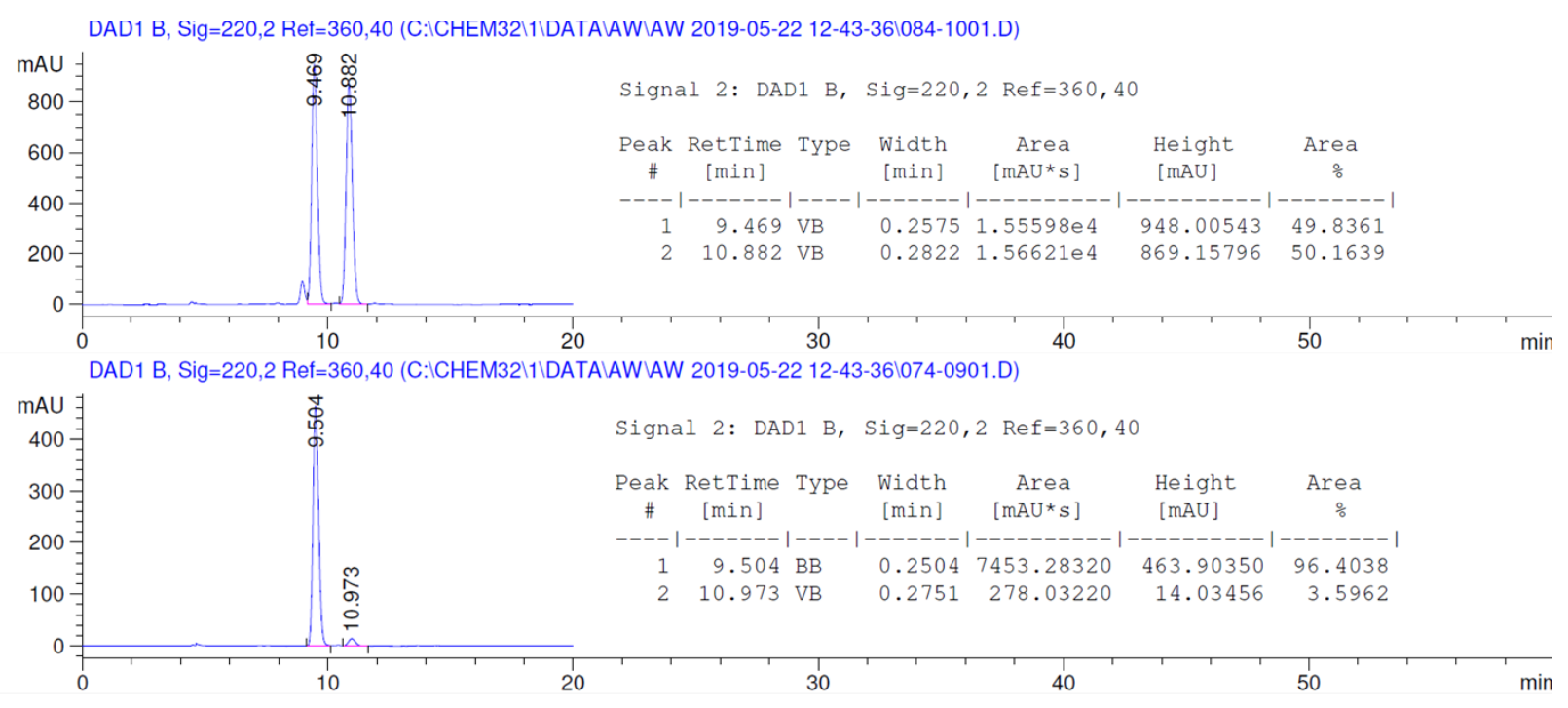


(1S,2R)-2-(3-methoxyphenyl)-1-phenyl-2-((4,4,5,5-tetramethyl-1,3,2-dioxaborolan-2yl)methyl)cyclobutan-1-ol (2x)<smiles>COc1cccc([C@]2(CBr)CC[C@@]2(O)c2ccccc2)c1</smiles>

Prepared following General Procedure 3. Product was isolated by flash column chromatography 0 -> 5\% acetone/pentane. Obtained $71 \mathrm{mg}$ of a white solid (90\% yield, 94:6 er).

${ }^{1} \mathbf{H}$ NMR $\left(500 \mathrm{MHz}, \mathrm{CDCl}_{3}\right) \delta 7.50(\mathrm{dd}, J=8.3,1.3 \mathrm{~Hz}, 2 \mathrm{H}), 7.37(\mathrm{dd}, J=8.3,7.0 \mathrm{~Hz}, 2 \mathrm{H}), 7.30$ $-7.24(\mathrm{~m}, 2 \mathrm{H}), 6.90(\mathrm{ddd}, J=7.7,1.8,1.0 \mathrm{~Hz}, 1 \mathrm{H}), 6.87(\mathrm{dd}, J=2.6,1.8 \mathrm{~Hz}, 1 \mathrm{H}), 6.78(\mathrm{ddd}, J=$ 8.2, 2.6, 1.0 Hz, 1H), $3.81(\mathrm{~s}, 3 \mathrm{H}), 3.00-2.87(\mathrm{~m}, 2 \mathrm{H}), 2.14-2.07(\mathrm{~m}, 2 \mathrm{H}), 2.05-1.98(\mathrm{~m}, 1 \mathrm{H})$, $1.03(\mathrm{~d}, J=14.9 \mathrm{~Hz}, 1 \mathrm{H}), 0.92(\mathrm{~m}, 7 \mathrm{H}$, overlap), $0.90(\mathrm{~s}, 6 \mathrm{H})$.

${ }^{13} \mathrm{C}$ NMR $\left(126 \mathrm{MHz}, \mathrm{CDCl}_{3}\right) \delta 159.6,144.5,142.7,129.2,127.9,127.0,126.5,120.1,113.5$, 112.0, 82.6, 82.3, 55.2, 54.1, 29.3, 26.5, 24.6, 24.4 .

IR (ATR) 3480, 2975, 1607, 1578, 1483, 1352, 1284, 1213, 1136, 1044, 957, 880, 862, 780

HRMS (DART, M+NH4) Calculated for $\mathrm{C}_{24} \mathrm{H}_{345} \mathrm{~B}_{1} \mathrm{~N}_{1} \mathrm{O}_{4} 412.2659$, found 412.2658

MP $52{ }^{\circ} \mathrm{C}$

$\boldsymbol{\alpha}_{\boldsymbol{D}}^{\mathbf{2 0}}\left(\mathrm{c}=0.265, \mathrm{CH}_{2} \mathrm{Cl}_{2}\right) 9.1$

HPLC: IA, 6\% IPA/Hex, $1 \mathrm{~mL} / \mathrm{min}$

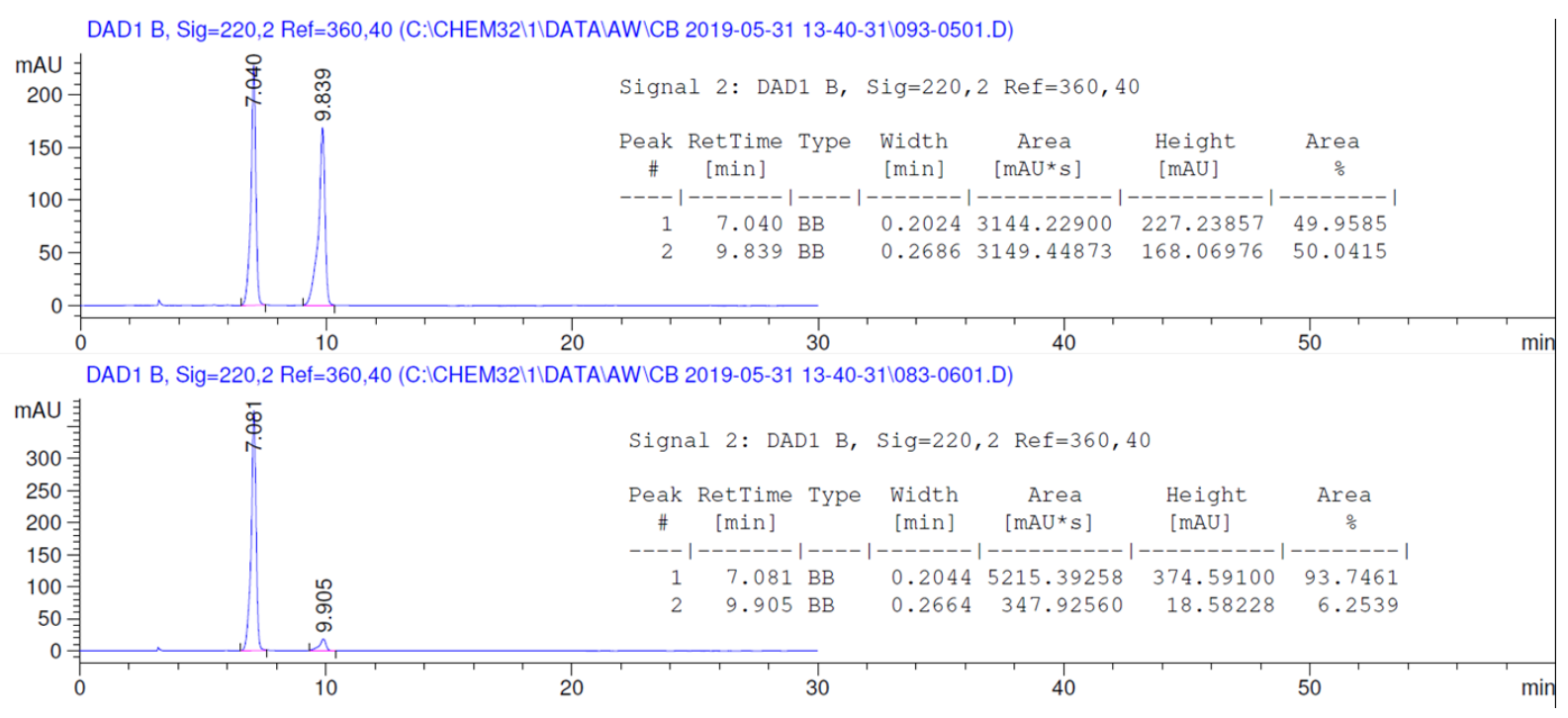


(1S,2R)-2-(3-fluorophenyl)-1-phenyl-2-((4,4,5,5-tetramethyl-1,3,2-dioxaborolan-2yl)methyl)cyclobutan-1-ol (2y)

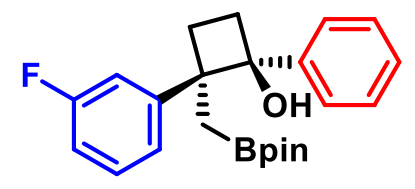

Prepared following General Procedure 3. Product was isolated by flash column chromatography 0 -> 5\% acetone/pentane. Obtained $65 \mathrm{mg}$ of a white solid (85\% yield, 95:5 er).

${ }^{1} \mathbf{H}$ NMR $\left(500 \mathrm{MHz}, \mathrm{CDCl}_{3}\right) \delta 7.48(\mathrm{dd}, J=8.3,1.3 \mathrm{~Hz}, 2 \mathrm{H}), 7.38(\mathrm{dd}, J=8.3,7.0 \mathrm{~Hz}, 2 \mathrm{H}), 7.34$ $-7.27(\mathrm{~m}, 2 \mathrm{H}), 7.12-7.03(\mathrm{~m}, 2 \mathrm{H}), 6.93(\mathrm{tdd}, J=8.3,2.6,1.0 \mathrm{~Hz}, 1 \mathrm{H}), 3.01-2.86(\mathrm{~m}, 2 \mathrm{H})$,

$2.11(\mathrm{ddd}, J=11.2,8.1,4.3 \mathrm{~Hz}, 1 \mathrm{H}), 2.00(\mathrm{ddd}, J=10.8,8.4,4.3 \mathrm{~Hz}, 1 \mathrm{H}), 1.94(\mathrm{~s}, 1 \mathrm{H}), 1.04$ (d, $J=15.1 \mathrm{~Hz}, 1 \mathrm{H}), 0.93(\mathrm{~s}, 6 \mathrm{H}), 0.90(\mathrm{~m}, 7 \mathrm{H}$, overlap).

${ }^{13}$ C NMR $\left(126 \mathrm{MHz}, \mathrm{CDCl}_{3}\right) \delta 162.9(\mathrm{~d}, J=245.1 \mathrm{~Hz}), 146.2(\mathrm{~d}, J=6.7 \mathrm{~Hz}), 142.4,129.5(\mathrm{~d}, J$ $=8.2 \mathrm{~Hz}), 128.0,127.2,126.4,123.3(\mathrm{~d}, J=2.8 \mathrm{~Hz}), 114.9(\mathrm{~d}, J=21.6 \mathrm{~Hz}), 113.2(\mathrm{~d}, J=21.0$ $\mathrm{Hz}), 82.7,82.5,53.8$ (d, $J=1.7 \mathrm{~Hz}), 29.4,26.5,24.6,24.4$.

${ }^{19}$ F NMR $\left(377 \mathrm{MHz}, \mathrm{CDCl}_{3}\right) \delta-113.4$.

IR (ATR) 3456, 2975, 2937, 1585, 1484, 1358, 1339, 1293, 1203, 1137, 948, 863, 783, 746, 707, 688, 578

HRMS (DART, M-H $\mathrm{H}_{2} \mathrm{O}+\mathrm{H}$ ) Calculated for $\mathrm{C}_{23} \mathrm{H}_{27} \mathrm{~B}_{1} \mathrm{~F}_{1} \mathrm{O}_{2}$ 365.2088, found 365.2094

MP $134{ }^{\circ} \mathrm{C}$

$\boldsymbol{\alpha}_{D}^{\mathbf{2 0}}\left(\mathrm{c}=0.32, \mathrm{CH}_{2} \mathrm{Cl}_{2}\right) 5.21$

HPLC: IA, 4\% IPA/Hex, $0.75 \mathrm{~mL} / \mathrm{min}$

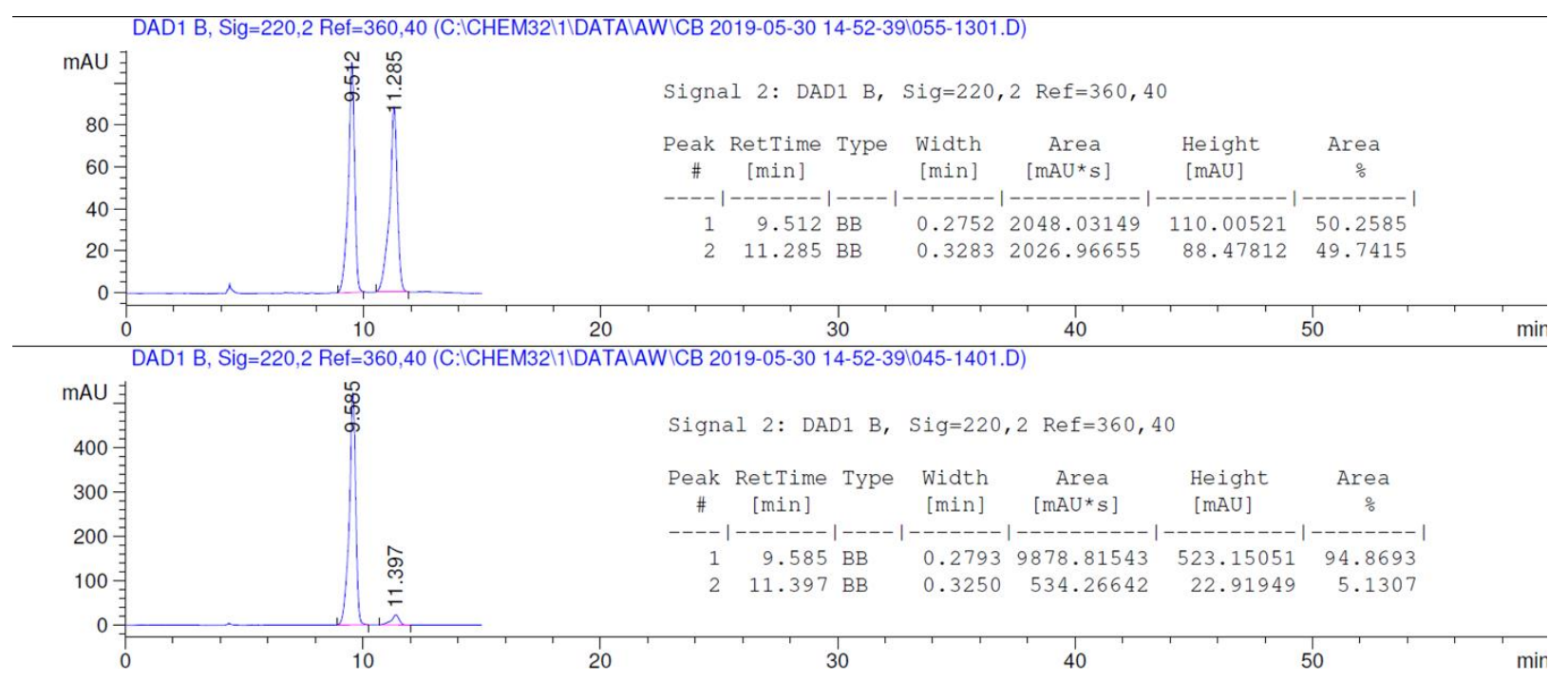


(1S,2R)-1-phenyl-2-((4,4,5,5-tetramethyl-1,3,2-dioxaborolan-2-yl)methyl)-2-(3(trifluoromethyl)phenyl)cyclobutan-1-ol (2z)<smiles>O[B][C@@]1(c2ccccc2)CC[C@@]1(CBr)c1cccc(C(F)(F)F)c1</smiles>

Prepared following General Procedure 3. Product was isolated by flash column chromatography 0 -> 5\% acetone/pentane. Obtained $69 \mathrm{mg}$ of a white solid (80\% yield, 95:5 er).

${ }^{1} \mathbf{H}$ NMR $\left(500 \mathrm{MHz}, \mathrm{CDCl}_{3}\right) \delta 7.61(\mathrm{~s}, 1 \mathrm{H}), 7.53-7.42(\mathrm{~m}, 5 \mathrm{H}), 7.40(\mathrm{dd}, J=8.4,6.9 \mathrm{~Hz}, 2 \mathrm{H})$, $7.34-7.27(\mathrm{~m}, 1 \mathrm{H}), 3.05-2.94(\mathrm{~m}, 2 \mathrm{H}), 2.18-2.11(\mathrm{~m}, 1 \mathrm{H}), 2.03-1.95(\mathrm{~m}, 1 \mathrm{H}), 1.86-1.82$ (m, 1H), 1.10 (d, J = 15.2 Hz, 1H), 0.89 (m, 7H, overlap), 0.87 (s, 6H).

${ }^{13} \mathrm{C}$ NMR $\left(126 \mathrm{MHz}, \mathrm{CDCl}_{3}\right) \delta 144.8,142.4,131.1(\mathrm{~d}, J=1.5 \mathrm{~Hz}), 130.2(\mathrm{q}, J=31.8 \mathrm{~Hz})$, $128.3,128.1,127.4,126.4,124.6(\mathrm{q}, J=3.8 \mathrm{~Hz}), 124.4(\mathrm{q}, J=272.4 \mathrm{~Hz}), 123.0(\mathrm{q}, J=3.7 \mathrm{~Hz})$, $82.7,82.7,53.6,29.4,26.4,24.5,24.3$.

${ }^{19} \mathbf{F}$ NMR $\left(377 \mathrm{MHz}, \mathrm{CDCl}_{3}\right) \delta-62.5$.

IR (ATR) 3470, 2983, 1356, 1326, 1269, 1158, 1139, 1118, 1074, 918, 817, 805, 701, 683, 575

HRMS (DART, $\mathrm{M}+\mathrm{NH}_{4}$ ) Calculated for $\mathrm{C}_{24} \mathrm{H}_{32} \mathrm{~B}_{1} \mathrm{~F}_{3} \mathrm{~N}_{1} \mathrm{O}_{3} 450.2427$, found 450.2421

MP $94{ }^{\circ} \mathrm{C}$

$\boldsymbol{\alpha}_{\boldsymbol{D}}^{\mathbf{2 0}}\left(\mathrm{c}=0.125, \mathrm{CH}_{2} \mathrm{Cl}_{2}\right) 9.6$

HPLC: IA, 4\% IPA/Hex, $0.75 \mathrm{~mL} / \mathrm{min}$

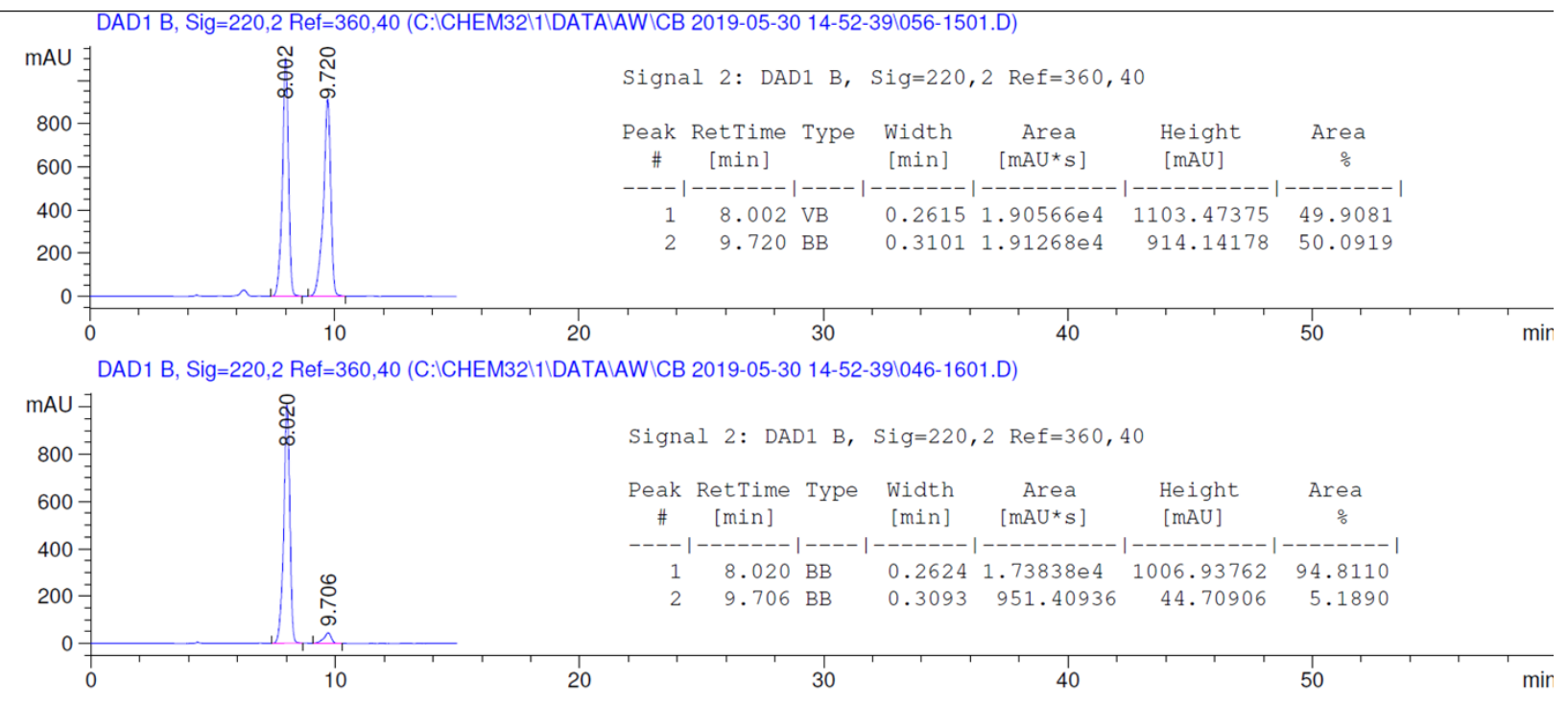


(1S,2R)-2-(3-chlorophenyl)-1-phenyl-2-((4,4,5,5-tetramethyl-1,3,2-dioxaborolan-2yl)methyl)cyclobutan-1-ol (2aa)<smiles>O[C@@]1(c2ccccc2)CC[C@@]1(C[Pb])c1cccc(Cl)c1</smiles>

Prepared following General Procedure 3. Product was isolated by flash column chromatography 0 -> 5\% acetone/pentane. Obtained $68 \mathrm{mg}$ of a white solid (85\% yield, 95:5 er).

${ }^{1} \mathbf{H}$ NMR $\left(500 \mathrm{MHz}, \mathrm{CDCl}_{3}\right) \delta 7.49(\mathrm{dd}, J=8.4,1.2 \mathrm{~Hz}, 2 \mathrm{H}), 7.38(\mathrm{td}, J=7.1,1.8 \mathrm{~Hz}, 2 \mathrm{H}), 7.35$ $(\mathrm{t}, J=1.9 \mathrm{~Hz}, 1 \mathrm{H}), 7.32-7.24(\mathrm{~m}, 2 \mathrm{H}), 7.21(\mathrm{ddd}, J=7.9,2.1,1.1 \mathrm{~Hz}, 1 \mathrm{H}), 7.17$ (ddd, $J=7.9$, $1.8,1.2 \mathrm{~Hz}, 1 \mathrm{H}), 3.01-2.85(\mathrm{~m}, 2 \mathrm{H}), 2.11(\mathrm{ddd}, J=11.1,7.9,4.2 \mathrm{~Hz}, 1 \mathrm{H}), 2.03-1.96(\mathrm{~m}, 1 \mathrm{H})$, $1.94(\mathrm{~s}, 1 \mathrm{H}), 1.05(\mathrm{~d}, J=15.1 \mathrm{~Hz}, 1 \mathrm{H}), 0.93(\mathrm{~s}, 6 \mathrm{H}), 0.91(\mathrm{~s}, 6 \mathrm{H}), 0.87$ (dd, $J=15.1,1.3 \mathrm{~Hz}, 1 \mathrm{H})$.

${ }^{13}$ C NMR $\left(126 \mathrm{MHz}, \mathrm{CDCl}_{3}\right) \delta 145.7,142.4,134.1,129.3,128.1,128.0,127.3,126.4,126.4$, $125.8,82.7,82.5,53.7,29.4,26.5,24.6,24.4$.

IR (ATR) 3441, 2979, 2198, 2142, 1596, 1355, 1334, 1269, 1213, 1136, 958, 881, 870, 847, $761,707,668,591$

HRMS (DART, $\mathrm{M}+\mathrm{NH}_{4}$ ) Calculated for $\mathrm{C}_{23} \mathrm{H}_{32} \mathrm{~B}_{1} \mathrm{Cl}_{1} \mathrm{~N}_{1} \mathrm{O}_{3} 416.2164$, found 416.2160

MP $98{ }^{\circ} \mathrm{C}$

$\boldsymbol{\alpha}_{\boldsymbol{D}}^{\mathbf{2 0}}\left(\mathrm{c}=0.17, \mathrm{CH}_{2} \mathrm{Cl}_{2}\right) 14.1$

HPLC: IA, $4 \%$ IPA/Hex, $0.75 \mathrm{~mL} / \mathrm{min}$
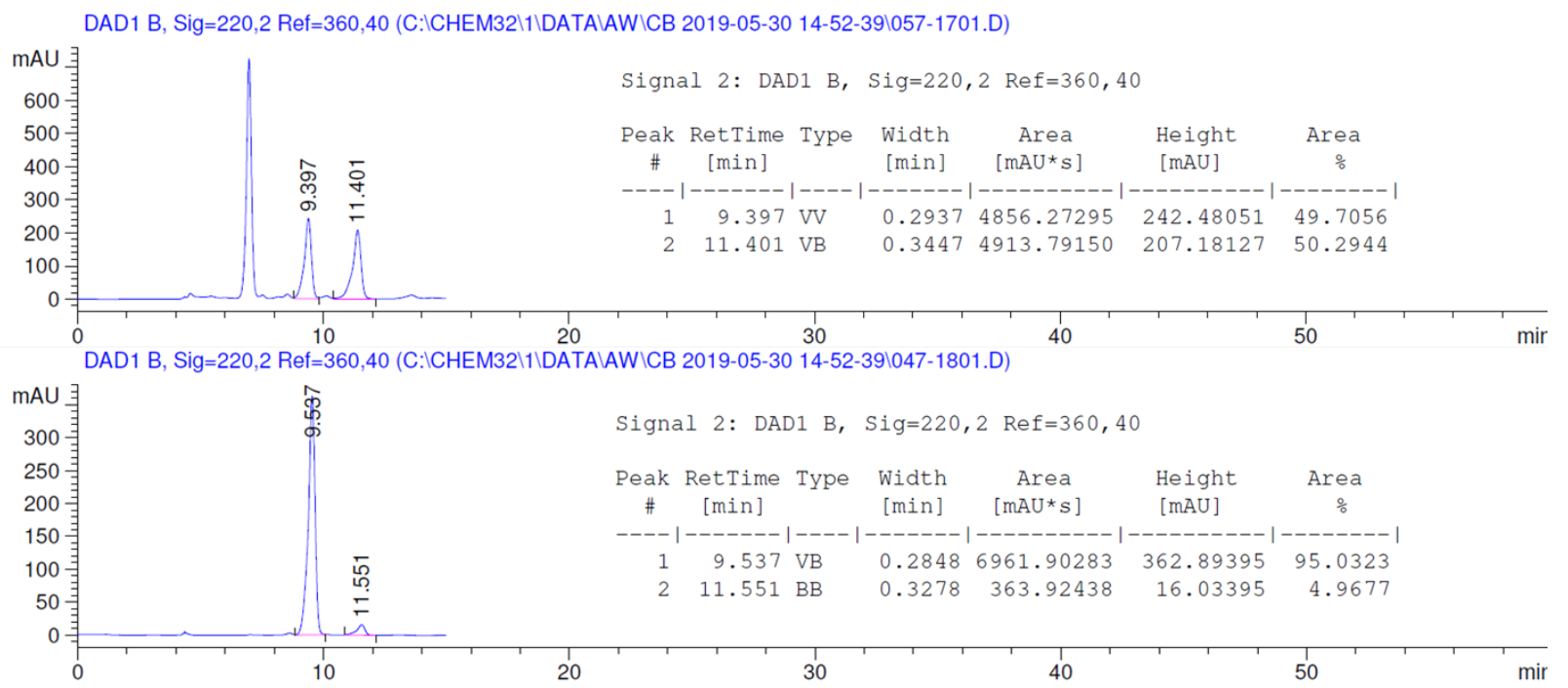
(1S,2S)-1-phenyl-2-((4,4,5,5-tetramethyl-1,3,2-dioxaborolan-2-yl)methyl)-2-(thiophen-2yl)cyclobutan-1-ol (2ab)

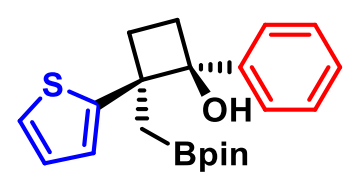

Prepared following General Procedure 3. Product was isolated by flash column chromatography $0->5 \%$ acetone/pentane. Obtained $61 \mathrm{mg}$ of a white solid (82\% yield, 93.5:6.5 er).

${ }^{1} \mathbf{H}$ NMR $\left(500 \mathrm{MHz}, \mathrm{CDCl}_{3}\right) \delta 7.44-7.41(\mathrm{~m}, 2 \mathrm{H}), 7.37-7.32(\mathrm{~m}, 2 \mathrm{H}), 7.28-7.24(\mathrm{~m}, 2 \mathrm{H})$, $7.07-6.99(\mathrm{~m}, 2 \mathrm{H}), 2.93(\mathrm{ddd}, J=12.1,8.6,7.5 \mathrm{~Hz}, 1 \mathrm{H}), 2.81$ (dddd, $J=11.3,8.8,7.5,1.4 \mathrm{~Hz}$, $1 \mathrm{H}), 2.25(\mathrm{~d}, J=1.8 \mathrm{~Hz}, 1 \mathrm{H}), 2.25-2.11(\mathrm{~m}, 2 \mathrm{H}), 0.99$ (s, 6H), $0.96(\mathrm{dd}, J=15.3,1.4 \mathrm{~Hz}, 1 \mathrm{H})$, $0.92(\mathrm{~s}, 6 \mathrm{H}), 0.87(\mathrm{~d}, J=15.3 \mathrm{~Hz}, 1 \mathrm{H})$.

${ }^{13} \mathrm{C}$ NMR $\left(126 \mathrm{MHz}, \mathrm{CDCl}_{3}\right) \delta 147.8,142.4,128.0,127.2,127.1,126.2,125.6,124.6,82.7$, $81.5,51.9,29.4,29.2,24.6,24.2$.

IR (ATR) 3466, 2976, 2937, 1684, 1448, 1356, 1326, 1273, 1214, 1137, 968, 846, 771, 689, 614

HRMS (DART, $\mathrm{M}+\mathrm{NH}_{4}$ ) Calculated for $\mathrm{C}_{21} \mathrm{H}_{31} \mathrm{~B}_{1} \mathrm{~N}_{1} \mathrm{O}_{3} \mathrm{~S}_{1} 388.2118$, found 388.2116

MP $54{ }^{\circ} \mathrm{C}$

$\boldsymbol{\alpha}_{D}^{20}\left(\mathrm{c}=0.11, \mathrm{CH}_{2} \mathrm{Cl}_{2}\right)-7.6$

HPLC: IC, $4 \%$ IPA/Hex, $0.75 \mathrm{~mL} / \mathrm{min}$

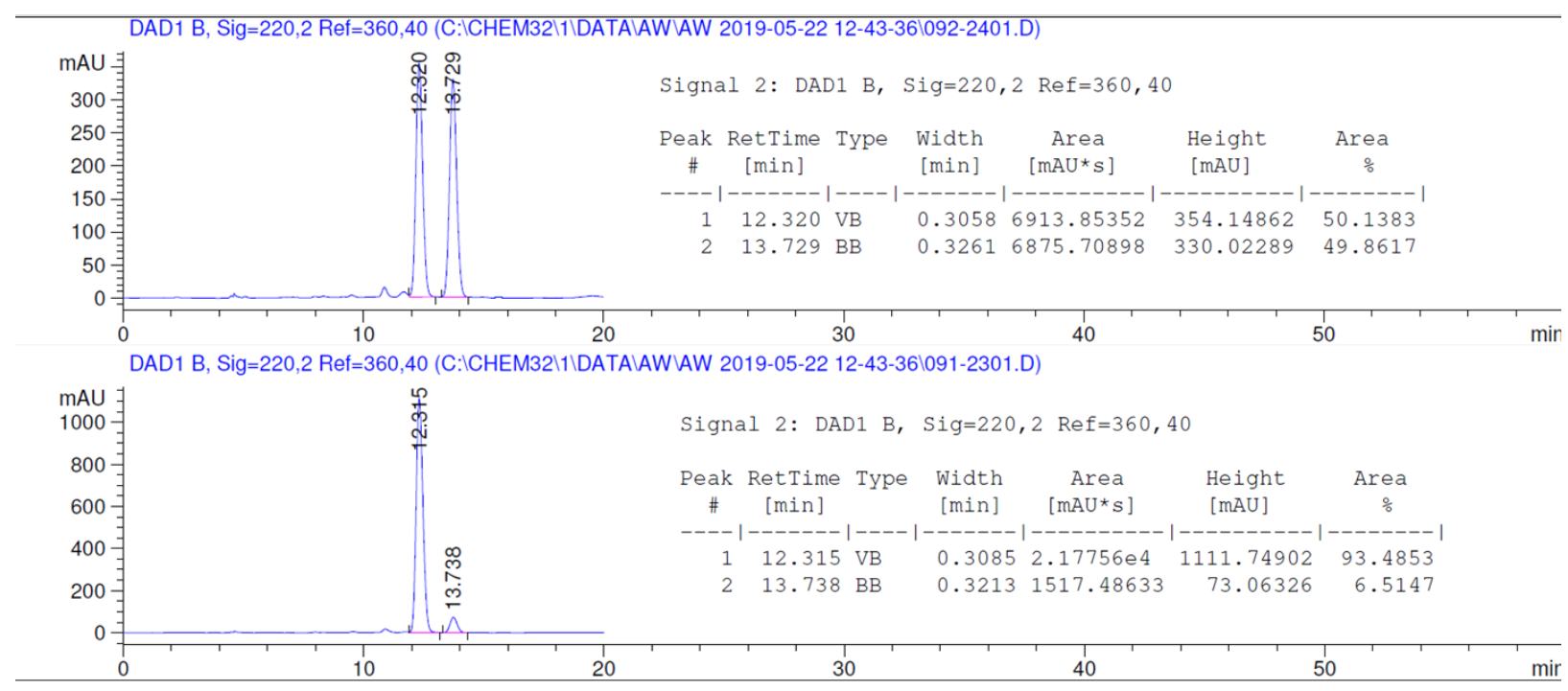


(1S,4R)-2,2-dimethyl-1,4-diphenyl-4-((4,4,5,5-tetramethyl-1,3,2-dioxaborolan-2yl)methyl)cyclobutan-1-ol (2ac)<smiles>CC1(C)C[C@@](CBr)(c2ccccc2)[C@@]1(O)c1ccccc1</smiles>

Prepared following General Procedure 3. Product was isolated by flash column chromatography 0 -> 5\% acetone/pentane. Obtained $69 \mathrm{mg}$ of a clear oil (88\% yield, 96.5:3.5 er).

${ }^{1} \mathbf{H}$ NMR $\left(500 \mathrm{MHz}, \mathrm{CDCl}_{3}\right) \delta 7.76-7.70(\mathrm{~m}, 2 \mathrm{H}), 7.39-7.33(\mathrm{~m}, 2 \mathrm{H}), 7.31(\mathrm{~s}, 4 \mathrm{H}), 7.29$ $7.26(\mathrm{~m}, 1 \mathrm{H}), 7.21-7.15(\mathrm{~m}, 1 \mathrm{H}), 2.81(\mathrm{dd}, J=11.9,1.4 \mathrm{~Hz}, 1 \mathrm{H}), 2.28(\mathrm{~d}, J=11.9 \mathrm{~Hz}, 1 \mathrm{H})$, $1.81-1.76$ (m, 2H, overlap), 1.53 (dd, $J=14.7,1.4 \mathrm{~Hz}, 1 \mathrm{H}), 1.30$ (s, 3H), 1.18 (s, 3H), 1.01 (s, $6 \mathrm{H}), 0.98$ (s, 6H).

${ }^{13}$ C NMR $\left(126 \mathrm{MHz}, \mathrm{CDCl}_{3}\right) \delta 145.5,143.0,128.2,128.0,127.5,126.6,126.0,83.5,82.7,51.8$, $42.3,40.6,27.9,25.9,24.7,24.6$.

IR (ATR) 3560, 2977, 2935, 1714, 1493, 1444, 1349, 1325, 1214, 1143, 1041, 967, 885, 846, $765,700,665$

HRMS (DART, M- $\mathrm{H}_{2} \mathrm{O}+\mathrm{H}$ ) Calculated for $\mathrm{C}_{25} \mathrm{H}_{32} \mathrm{~B}_{1} \mathrm{O}_{2} 375.2495$, found 375.2498 $\boldsymbol{\alpha}_{\boldsymbol{D}}^{\mathbf{2 0}}\left(\mathrm{c}=0.20, \mathrm{CH}_{2} \mathrm{Cl}_{2}\right) 91.9$

HPLC: IA, 4\% IPA/Hex, $0.75 \mathrm{~mL} / \mathrm{min}$

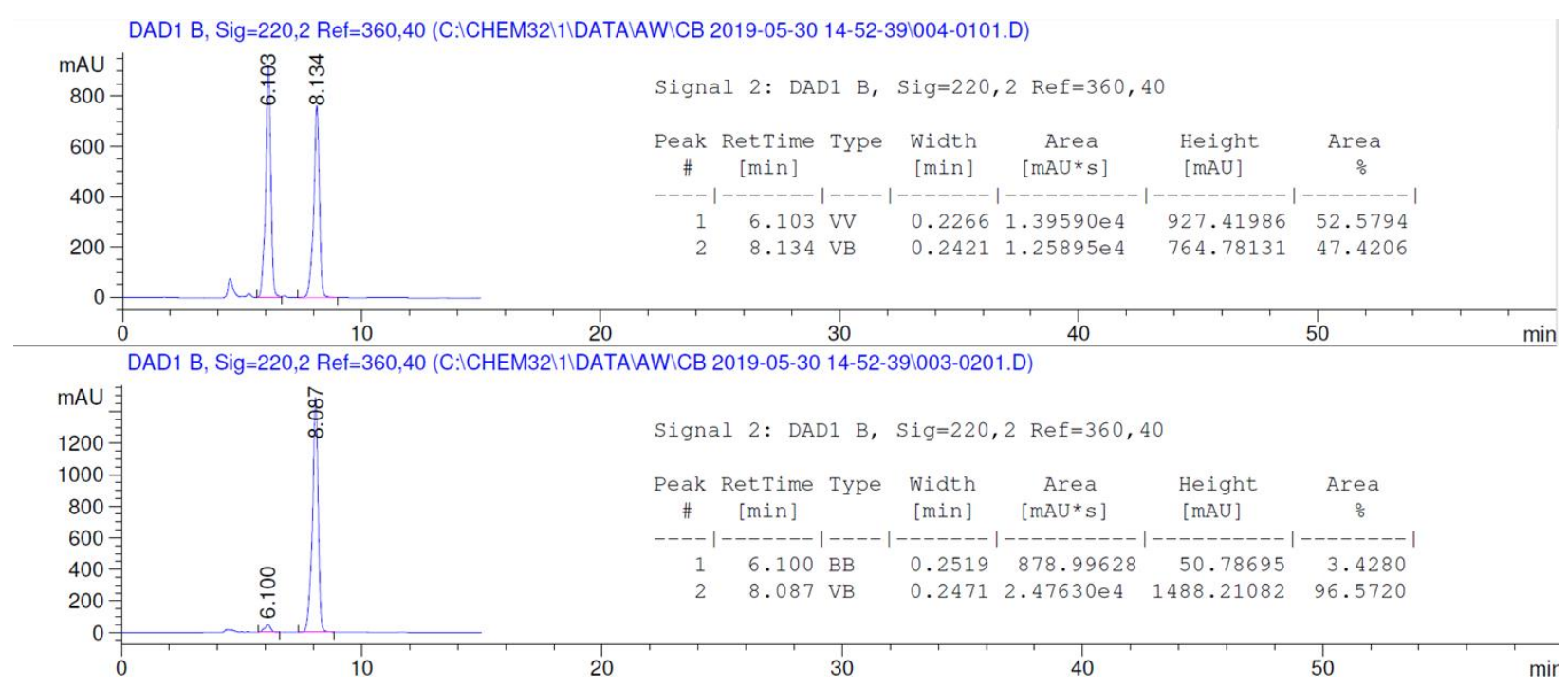


(1S,2R)-1,2-diphenyl-2-((4,4,5,5-tetramethyl-1,3,2-dioxaborolan-2-

yl)methyl)spiro[3.5]nonan-1-ol (2ad)

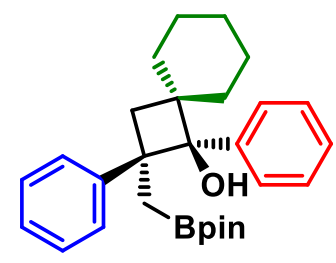

Prepared following General Procedure 3. Product was isolated by flash column chromatography 10 -> 50\% DCM/pentane. Obtained $52 \mathrm{mg}$ of a clear oil (60\% yield, 96:4 er).

${ }^{1} \mathbf{H}$ NMR $\left(500 \mathrm{MHz}, \mathrm{CDCl}_{3}\right) \delta 7.84-7.76(\mathrm{~m}, 2 \mathrm{H}), 7.40-7.33(\mathrm{~m}, 2 \mathrm{H}), 7.33-7.21(\mathrm{~m}, 5 \mathrm{H})$, $7.20-7.14(\mathrm{~m}, 1 \mathrm{H}), 2.77(\mathrm{dd}, J=12.2,1.2 \mathrm{~Hz}, 1 \mathrm{H}), 2.32(\mathrm{~d}, J=12.2 \mathrm{~Hz}, 1 \mathrm{H}), 2.01(\mathrm{dd}, J=$ 13.5, 3.3 Hz, 1H), 1.85 (dd, $J=12.1,3.6 \mathrm{~Hz}, 1 \mathrm{H}), 1.81$ (d, $J=14.5 \mathrm{~Hz}, 1 \mathrm{H}), 1.76(\mathrm{~s}, 1 \mathrm{H}), 1.63-$ $1.21(\mathrm{~m}, 8 \mathrm{H}), 1.15-1.04(\mathrm{~m}, 1 \mathrm{H}), 1.01(\mathrm{~s}, 6 \mathrm{H}), 0.96(\mathrm{~s}, 6 \mathrm{H})$.

${ }^{13} \mathrm{C}$ NMR $\left(126 \mathrm{MHz}, \mathrm{CDCl}_{3}\right) \delta 145.5,142.4,128.2,128.2,127.8,127.3,126.5,126.0,83.1$, 82.6, 51.8, 45.4, 38.1, 35.3, 33.4, 26.1, 24.7, 24.7, 22.9, 22.6.

IR (ATR) 3561, 2976, 2926, 2854, 1602, 1495, 1449, 1356, 1331, 1277, 1210, 1149, 1110, $1015,971,840,723,703$

HRMS (ESI, M+Na) Calculated for $\mathrm{C}_{28} \mathrm{H}_{37} \mathrm{BNaO}_{3} 454.2764$, found 454.2760

$\boldsymbol{\alpha}_{\boldsymbol{D}}^{\mathbf{2 0}}\left(\mathrm{c}=0.16, \mathrm{CH}_{2} \mathrm{Cl}_{2}\right) 41.2$

HPLC: IA, $1 \%$ IPA/Hex, $0.5 \mathrm{~mL} / \mathrm{min}$

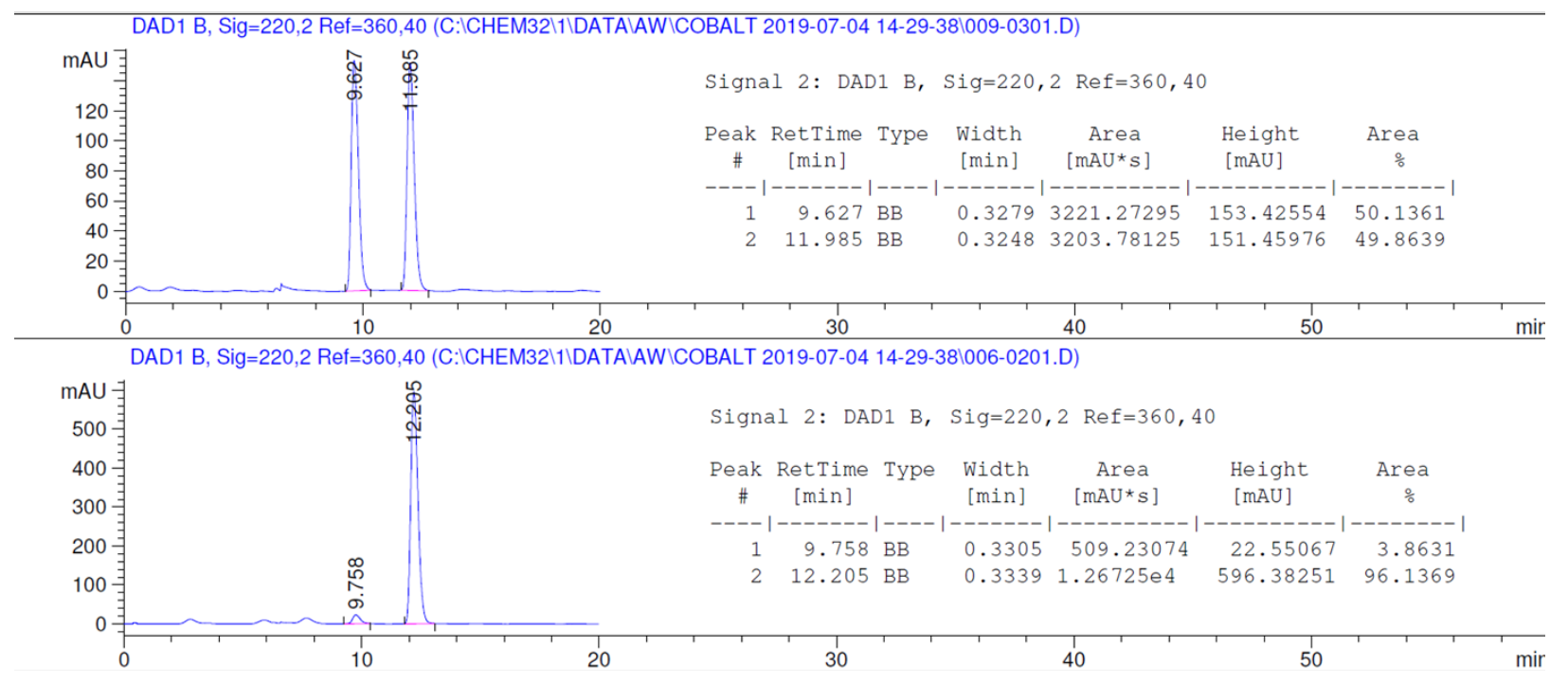


(1R,2R)-1-(2-chlorophenyl)-2-phenyl-2-((4,4,5,5-tetramethyl-1,3,2-dioxaborolan-2yl)methyl)spiro[3.4]octan-1-ol (2ae)<smiles>O[C@@]1(c2ccccc2Cl)CC2(CCCC2)[C@@]1(O)c1ccccc1</smiles>

Prepared following General Procedure 3. Product was isolated by flash column chromatography $10->50 \%$ DCM/pentane. Obtained $34 \mathrm{mg}$ of a clear oil (38\% yield, 97:3 er).

${ }^{1} \mathbf{H}$ NMR $\left(500 \mathrm{MHz}, \mathrm{CDCl}_{3}\right) \delta 7.87(\mathrm{dd}, J=7.9,1.7 \mathrm{~Hz}, 1 \mathrm{H}), 7.56(\mathrm{dd}, J=8.5,1.2 \mathrm{~Hz}, 2 \mathrm{H}), 7.36$ $(\mathrm{dd}, J=7.8,1.5 \mathrm{~Hz}, 1 \mathrm{H}), 7.33(\mathrm{dd}, J=8.2,7.4 \mathrm{~Hz}, 2 \mathrm{H}), 7.27-7.19(\mathrm{~m}, 1 \mathrm{H}), 7.23-7.14(\mathrm{~m}$, $2 \mathrm{H}), 2.82(\mathrm{dd}, J=11.8,1.4 \mathrm{~Hz}, 1 \mathrm{H}), 2.68(\mathrm{~s}, 1 \mathrm{H}), 2.36-2.30(\mathrm{~m}, 1 \mathrm{H}), 2.33-2.26(\mathrm{~m}, 1 \mathrm{H}), 2.07$ (ddd, $J=12.9,8.6,7.1 \mathrm{~Hz}, 1 \mathrm{H}), 1.91$ (ddd, $J=12.9,7.5,5.5 \mathrm{~Hz}, 1 \mathrm{H}), 1.74-1.51(\mathrm{~m}, 6 \mathrm{H}), 1.39$ $(\mathrm{d}, J=15.1 \mathrm{~Hz}, 1 \mathrm{H}), 1.03(\mathrm{~s}, 6 \mathrm{H}), 1.02(\mathrm{~s}, 6 \mathrm{H})$.

${ }^{13} \mathbf{C}$ NMR $\left(126 \mathrm{MHz}, \mathrm{CDCl}_{3}\right) \delta 144.5,140.2,133.1,131.9,130.9,128.7,128.1,127.8,125.9$, 125.7, 87.9, 82.7, 52.5, 52.2, 46.4, 38.0, 37.9, 26.4, 24.8, 24.6, 24.3.

IR (ATR) 2983, 2869, 1945, 1470, 1349, 1145, 1092, 971, 849, 764, 748, 699

HRMS (ESI, M+NH 4 ) Calculated for $\mathrm{C}_{27} \mathrm{H}_{38} \mathrm{~B}_{1} \mathrm{Cl}_{1} \mathrm{~N}_{1} \mathrm{O}_{3} 469.2664$, found 469.2664

$\boldsymbol{\alpha}_{\boldsymbol{D}}^{\mathbf{2 0}}\left(\mathrm{c}=0.25, \mathrm{CH}_{2} \mathrm{Cl}_{2}\right) 23.5$

HPLC: OD-H, 1\% IPA/Hex, $0.3 \mathrm{~mL} / \mathrm{min}$
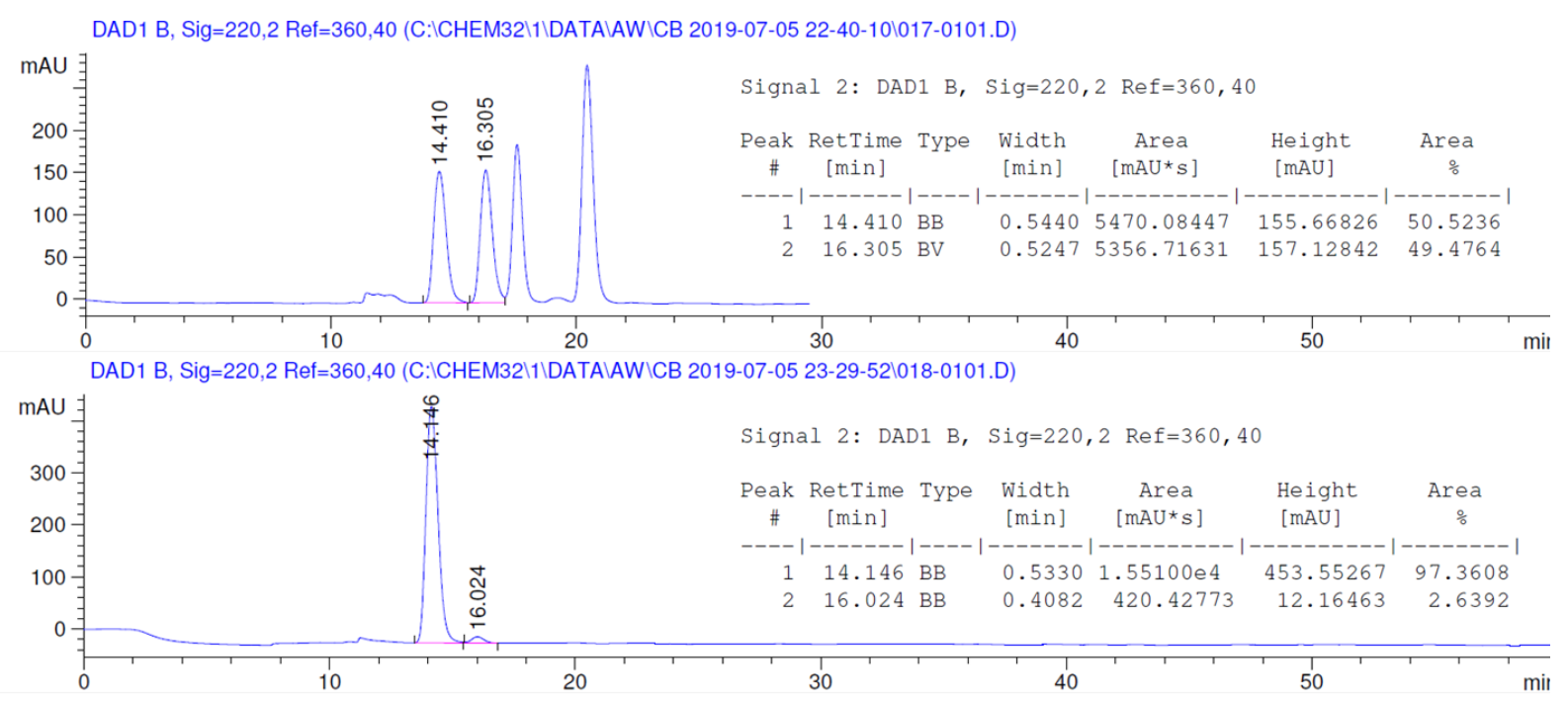


\section{Product Derivatizations}

\section{((1R,2S)-2-hydroxy-1,2-diphenylcyclobutyl)methyl benzoate (3a)}

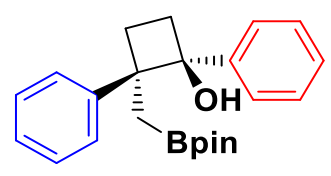

$2 e$

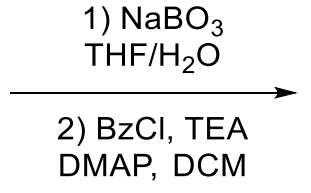

Scheme S3. Oxidation and benzoylation procedure

Product 2e (146 mg, $0.4 \mathrm{mmol})$ was dissolved in THF (2.5 mL) and water $(2.5 \mathrm{~mL})$. Sodium perborate (308 mg, $2 \mathrm{mmol}, 5$ equiv) was added. The oxidation was stirred for $16 \mathrm{~h}$ at room temperature. The reaction was filtered over a silica pad with ethyl acetate then washed with three times with water. The organic layer was dried over magnesium sulfate and filtered. The filtrate was concentrated to dryness. The crude was redissolved in DCM (4 mL). DMAP (4.8 mg, 0.04 mmol, 0.1 equiv) and TEA ( $0.11 \mathrm{~mL}, 0.8 \mathrm{mmol}, 2$ equiv) were added followed by a slow addition of benzoyl chloride $(0.11 \mathrm{~mL}, 0.6 \mathrm{mmol}, 1.5$ equiv). The acylation was stirred for $16 \mathrm{~h}$ at room temperature. The reaction was filtered over a silica pad and concentrated. The crude was purified by flash column chromatography using 60\% DCM/pentanes. Obtained $65 \mathrm{mg}$ of a clear oil (46\% yield, 95.5:4.5 er).

${ }^{1} \mathbf{H}$ NMR $\left(500 \mathrm{MHz}, \mathrm{CDCl}_{3}\right) \delta 7.70-7.58(\mathrm{~m}, 4 \mathrm{H}), 7.51-7.38(\mathrm{~m}, 7 \mathrm{H}), 7.35-7.27(\mathrm{~m}, 4 \mathrm{H})$, $4.43(\mathrm{~d}, J=11.4 \mathrm{~Hz}, 1 \mathrm{H}), 4.23(\mathrm{dd}, J=11.4,1.2 \mathrm{~Hz}, 1 \mathrm{H}), 3.11(\mathrm{dt}, J=12.4,8.8 \mathrm{~Hz}, 1 \mathrm{H}), 2.93$ (dddd, $J=11.6,9.6,8.4,1.2 \mathrm{~Hz}, 1 \mathrm{H}), 2.37-2.20(\mathrm{~m}, 2 \mathrm{H}), 2.20-2.08(\mathrm{~m}, 1 \mathrm{H})$.

${ }^{13} \mathrm{C}$ NMR $\left(126 \mathrm{MHz}, \mathrm{CDCl}_{3}\right) \delta 166.3,142.0,139.9,132.8,129.9,129.4,128.6,128.5,128.2$, $127.7,127.7,127.2,126.1,80.1,69.5,56.0,30.2,23.6$.

IR (ATR) 3482, 3061, 2954, 1713, 1602, 1584, 1499, 1449, 1377, 1270, 1117, 1024, 938, 774

HRMS (ESI, M+Na) Calculated for $\mathrm{C}_{24} \mathrm{H}_{22} \mathrm{NaO}_{3} 381.1461$, found 381.1460

$\boldsymbol{\alpha}_{\boldsymbol{D}}^{\mathbf{2 0}}\left(\mathrm{c}=0.195, \mathrm{CH}_{2} \mathrm{Cl}_{2}\right) 3.8$

HPLC: OD-H, $1 \%$ IPA/Hex, $0.5 \mathrm{~mL} / \mathrm{min}$

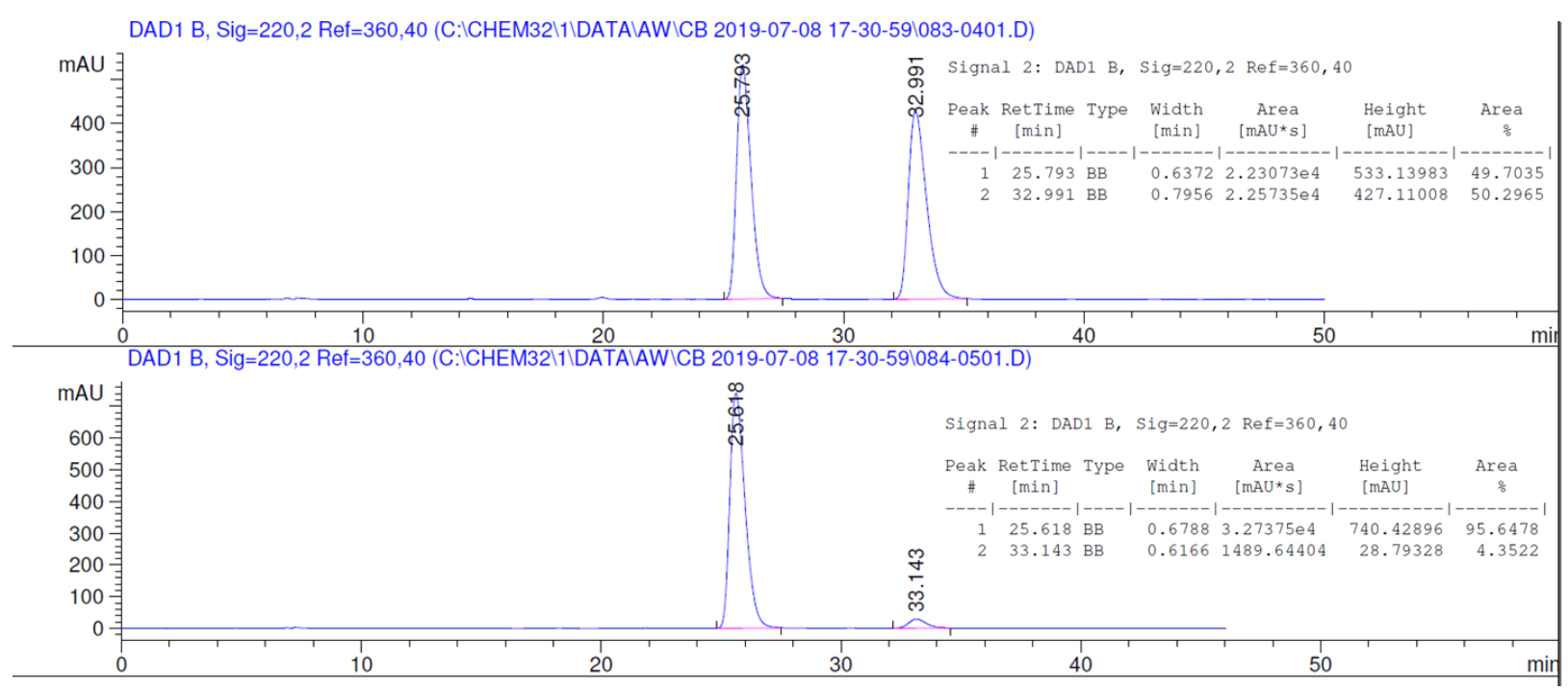


tert-butyl((1S,2R)-1,2-diphenyl-2-((4,4,5,5-tetramethyl-1,3,2-dioxaborolan-2yl)methyl)cyclobutoxy)dimethylsilane (3b)

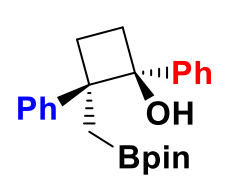

$2 e$

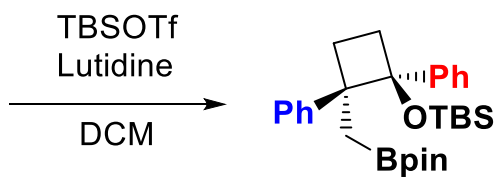

$3 \mathbf{b}$

Scheme S4. TBS protection

Product 2e (364 mg, $1 \mathrm{mmol}$ ) was dissolved in anhydrous DCM (5 mL). Distilled lutidine (1.16 $\mathrm{mL}, 10 \mathrm{mmol}, 10$ equiv) was added. The reaction was cooled to $0{ }^{\circ} \mathrm{C}$ and TBSOTf $(1.15 \mathrm{~mL}, 5$ mmol, 5 equiv) was added dropwise. The reaction was stirred for $2 \mathrm{~h}$ at room temperature. Then the reaction was concentrated under reduced pressure. The product was isolated by flash column chromatography 20 -> 50\% DCM/pentane. The product was obtained as a white solid (438 mg, 91\% yield, 95.5:4.5 er).

${ }^{1} \mathbf{H}$ NMR $\left(500 \mathrm{MHz}, \mathrm{CDCl}_{3}\right) \delta 7.49-7.45(\mathrm{~m}, 2 \mathrm{H}), 7.39-7.35(\mathrm{~m}, 2 \mathrm{H}), 7.35-7.30(\mathrm{~m}, 2 \mathrm{H})$, $7.30-7.21(\mathrm{~m}, 3 \mathrm{H}), 7.15-7.10(\mathrm{~m}, 1 \mathrm{H}), 2.96-2.81(\mathrm{~m}, 2 \mathrm{H}), 2.18-2.09(\mathrm{~m}, 1 \mathrm{H}), 1.96-1.84$ $(\mathrm{m}, 1 \mathrm{H}), 1.02(\mathrm{~d}, J=14.9 \mathrm{~Hz}, 1 \mathrm{H}), 0.87(\mathrm{~s}, 6 \mathrm{H}), 0.84(\mathrm{~s}, 6 \mathrm{H}), 0.60(\mathrm{~d}, J=14.9 \mathrm{~Hz}, 1 \mathrm{H}), 0.56(\mathrm{~s}$, $9 \mathrm{H}),-0.25$ (s, 3H), -0.48 (s, 3H).

${ }^{13} \mathrm{C}$ NMR $\left(126 \mathrm{MHz}, \mathrm{CDCl}_{3}\right) \delta 143.9,143.8,128.1,127.5,127.1,127.0,126.7,125.2,83.7$, $82.4,54.7,29.1,25.9,25.6,24.6,24.2,17.9,-3.1,-3.4$.

IR (ATR) 3065, 2936, 2858, 1606, 1502, 1474, 1452, 1395, 1363, 1327, 1252, 1145, 1078, 1003, 892

HRMS (ESI, M+Na) Calculated for $\mathrm{C}_{29} \mathrm{H}_{43} \mathrm{~B}_{1} \mathrm{Na}_{1} \mathrm{O}_{3} \mathrm{Si}_{1} 500.3003$, found 500.3002

MP $85{ }^{\circ} \mathrm{C}$

$\boldsymbol{\alpha}_{\boldsymbol{D}}^{\mathbf{2 0}}\left(\mathrm{c}=0.180, \mathrm{CH}_{2} \mathrm{Cl}_{2}\right) 19.6$

HPLC: IC, $0.25 \%$ IPA/Hex, $0.75 \mathrm{~mL} / \mathrm{min}$

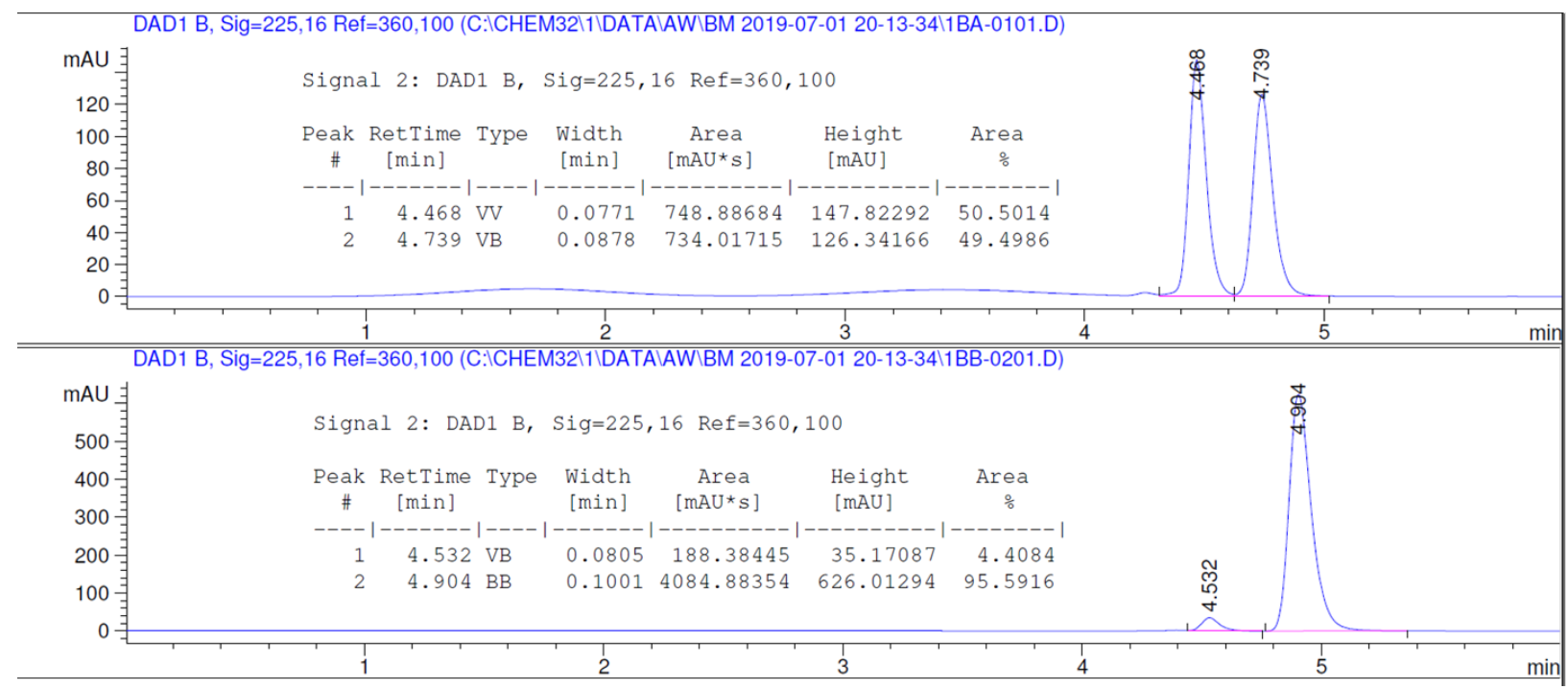


((1R,2S)-2-((tert-butyldimethylsilyl)oxy)-1,2-diphenylcyclobutyl)methanol (3c)

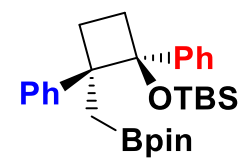

3b

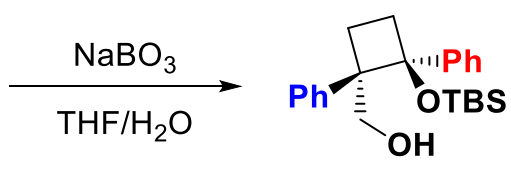

3c

Scheme S5. Oxidation procedure

Compound 3b (96 mg, $0.2 \mathrm{mmol}$ ) was dissolved in THF (1 mL) and $\mathrm{H}_{2} \mathrm{O}(1 \mathrm{~mL})$. Sodium perborate (154 mg, $1.0 \mathrm{mmol}, 5$ equiv) was added. The reaction was stirred for $16 \mathrm{~h}$ at room temperature. Another charge of sodium perborate $(154 \mathrm{mg}, 1.0 \mathrm{mmol}, 5$ equiv) was added followed by THF $(1 \mathrm{~mL})$. The reaction was stirred for another $12 \mathrm{~h}$. TLC indicated complete conversion of the boronate ester. The crude was filtered over silica gel then washed with three times with water. The organic layer was dried over magnesium sulfate and dried under reduced pressure. The product was purified by flash column chromatography in 0-> 5\% EtOAc/pentane. Obtained a white solid, $56 \mathrm{mg}$ (76\% yield, 96:4 er)

${ }^{1} \mathbf{H}$ NMR $\left(500 \mathrm{MHz}, \mathrm{CDCl}_{3}\right) \delta 7.57-7.51(\mathrm{~m}, 2 \mathrm{H}), 7.45-7.36(\mathrm{~m}, 6 \mathrm{H}), 7.31(\mathrm{t}, J=7.3 \mathrm{~Hz}$, 1H), $7.27-7.23(\mathrm{~m}, 1 \mathrm{H}), 3.45(\mathrm{~d}, J=11.3 \mathrm{~Hz}, 1 \mathrm{H}), 3.28(\mathrm{dd}, J=11.3,9.1 \mathrm{~Hz}, 1 \mathrm{H}), 2.97$ (ddd, $J$ $=12.1,8.8,7.0 \mathrm{~Hz}, 1 \mathrm{H}), 2.73(\mathrm{dddd}, J=11.1,9.3,7.0,1.2 \mathrm{~Hz}, 1 \mathrm{H}), 2.19$ (ddd, $J=12.1,9.3,5.9$ $\mathrm{Hz}, 1 \mathrm{H}), 2.10$ (ddd, $J=11.1,8.8,5.9 \mathrm{~Hz}, 1 \mathrm{H}), 0.71(\mathrm{~s}, 1 \mathrm{H}), 0.59(\mathrm{~s}, 9 \mathrm{H}),-0.20(\mathrm{~s}, 3 \mathrm{H}),-0.44$ (s, $3 \mathrm{H})$.

${ }^{13} \mathrm{C}$ NMR $\left(126 \mathrm{MHz}, \mathrm{CDCl}_{3}\right) \delta 142.8,140.3,128.4,128.0,127.9,127.4,126.6,126.3,81.8$, $68.8,58.8,29.3,25.6,22.3,18.0,-3.0,-3.3$.

IR (ATR) 3329, 2958, 2936, 2890, 2854, 1606, 1502, 1477, 1413, 1259, 1163, 1135, 1079, 924, 832

HRMS (ESI, M+Na) Calculated for $\mathrm{C}_{23} \mathrm{H}_{32} \mathrm{Na}_{1} \mathrm{O}_{2} \mathrm{Si}_{1} 391.2064$, found 391.2060

MP $88^{\circ} \mathrm{C}$

$\boldsymbol{\alpha}_{\boldsymbol{D}}^{\mathbf{2 0}}\left(\mathrm{c}=0.175, \mathrm{CH}_{2} \mathrm{Cl}_{2}\right) 49.1$

HPLC: IA, $1 \%$ IPA/Hex, $0.5 \mathrm{~mL} / \mathrm{min}$

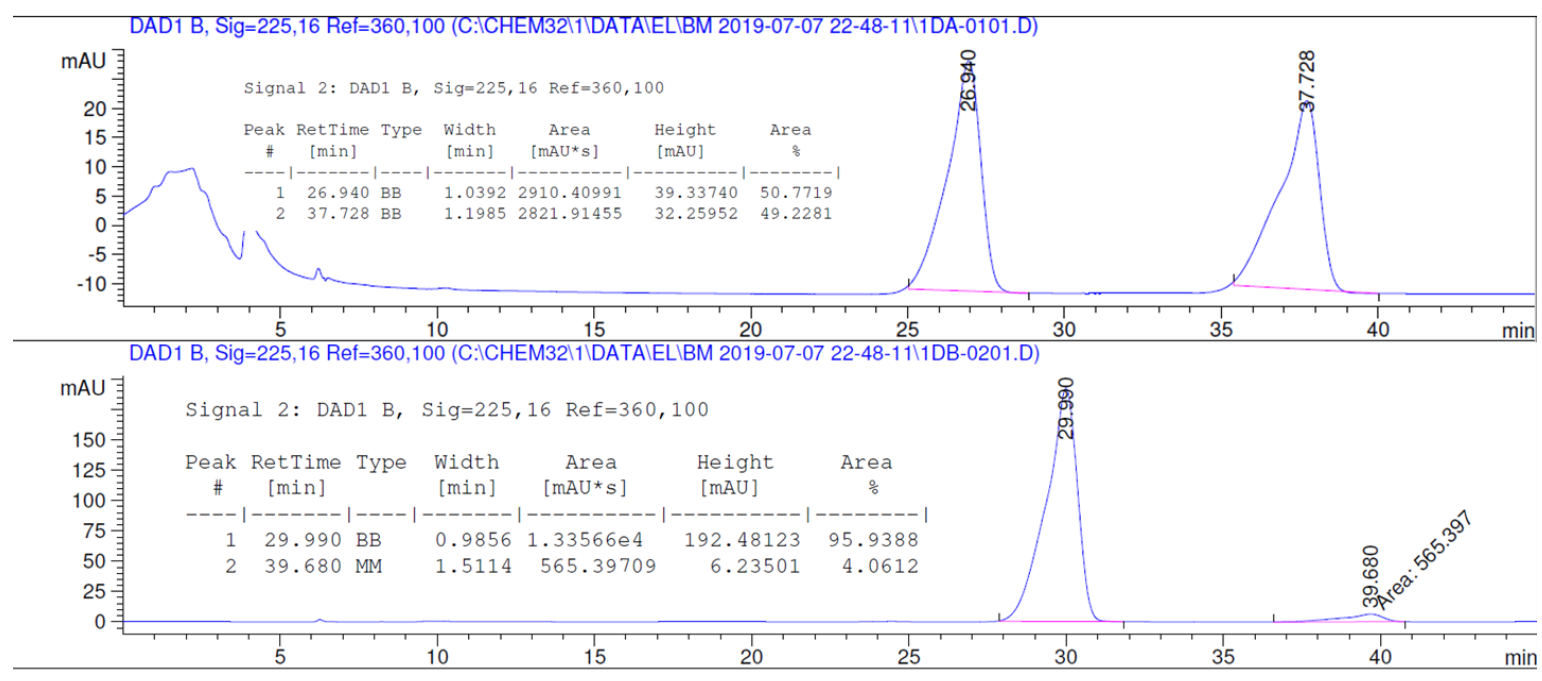




\section{2-((1S,2S)-2-((tert-butyldimethylsilyl)oxy)-1,2-diphenylcyclobutyl)ethan-1-ol (3d)}

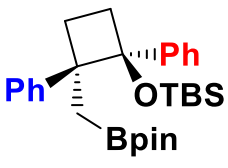

3b

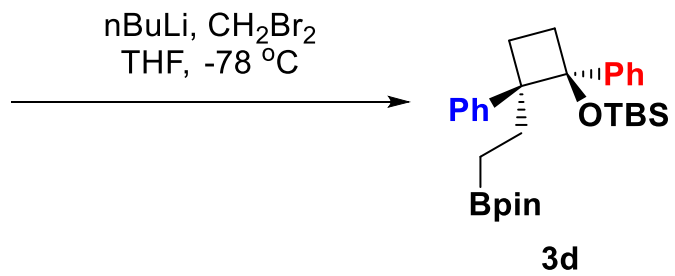

Scheme S6. Matteson homologation procedure

To a solution of product $3 \mathbf{b}$ (95 mg, $0.2 \mathrm{mmol}, 1$ equiv) and dibromomethane ( $35 \mu \mathrm{L}, 0.5 \mathrm{mmol}$, 2.5 equiv) in THF ( $2 \mathrm{~mL})$ at $-78^{\circ} \mathrm{C}$ was added $n$-butyllithium solution $(2.5 \mathrm{M}$ in hexanes, 0.176 $\mathrm{mL}, 2.2$ equiv) slowly down the side of the reaction vessel. After stirring at $-78{ }^{\circ} \mathrm{C}$ for an additional $20 \mathrm{~min}$, the cooling bath was removed and the reaction allowed to slowly warm to room temperature and stirred for $6 \mathrm{~h}$ at r.t. The resulting mixture was cooled to $0{ }^{\circ} \mathrm{C}$ and saturated ammonium chloride solution $(2 \mathrm{~mL})$ was slowly added. The mixture was diluted with ethyl acetate $(5 \mathrm{~mL})$. The aqueous layer was extracted with ethyl acetate and the combined organic layers were dried over anhydrous sodium sulfate, filtered, and concentrated under reduced pressure. The crude material was purified by flash column chromatography with $0->$ $50 \% \mathrm{DCM} /$ pentane to afford the product 3d (66 mg, 67\% yield, 96:4 er)

${ }^{1} \mathbf{H}$ NMR $\left(500 \mathrm{MHz}, \mathrm{CDCl}_{3}\right) \delta 7.55-7.50(\mathrm{~m}, 2 \mathrm{H}), 7.38-7.23(\mathrm{~m}, 7 \mathrm{H}), 7.18-7.11(\mathrm{~m}, 1 \mathrm{H})$, 2.86 (ddd, $J=12.0,8.7,6.6 \mathrm{~Hz}, 1 \mathrm{H}$ ), 2.67 (dddd, $J=11.0,9.1,6.6,1.2 \mathrm{~Hz}, 1 \mathrm{H}), 2.08$ (ddd, $J=$ 12.0, 9.1, 6.1 Hz, 1H), 1.77 (ddd, $J=11.0,8.7,6.1 \mathrm{~Hz}, 1 \mathrm{H}), 1.47-1.37(\mathrm{~m}, 1 \mathrm{H}), 1.20$ (ddd, $J=$ 13.7, 11.6, 5.0 Hz, 1H), 1.09 (s, 6H), 1.08 (s, 6H), 0.56 (s, 9H), 0.34 (ddd, J = 16.3, 11.6, $4.9 \mathrm{~Hz}$, $1 \mathrm{H}), 0.11-0.03(\mathrm{~m}, 1 \mathrm{H}),-0.26(\mathrm{~s}, 3 \mathrm{H}),-0.48(\mathrm{~s}, 3 \mathrm{H})$.

${ }^{13} \mathrm{C}$ NMR $\left(126 \mathrm{MHz}, \mathrm{CDCl}_{3}\right) \delta 143.7,142.6,128.3,127.5,127.3,127.1,126.7,125.2,83.1$, 82.6, 59.0, 32.5, 29.1, 25.6, 24.7, 24.6, 23.4, 17.9, -3.0, -3.3.

IR (ATR) 2979, 2958, 2933, 2854, 1606, 1449, 1370, 1320, 1252, 1145, 1003, 892, 835, 774

HRMS (ESI, M+Na) Calculated for $\mathrm{C}_{20} \mathrm{H}_{45} \mathrm{~B}_{1} \mathrm{Na}_{1} \mathrm{O}_{3} \mathrm{Si}_{1} 514.3160$, found 514.3143

$\boldsymbol{\alpha}_{\boldsymbol{D}}^{\mathbf{2 0}}\left(\mathrm{c}=0.180, \mathrm{CH}_{2} \mathrm{Cl}_{2}\right)-1.8$

HPLC: IC, $0.25 \%$ IPA/Hex, $0.75 \mathrm{~mL} / \mathrm{min}$
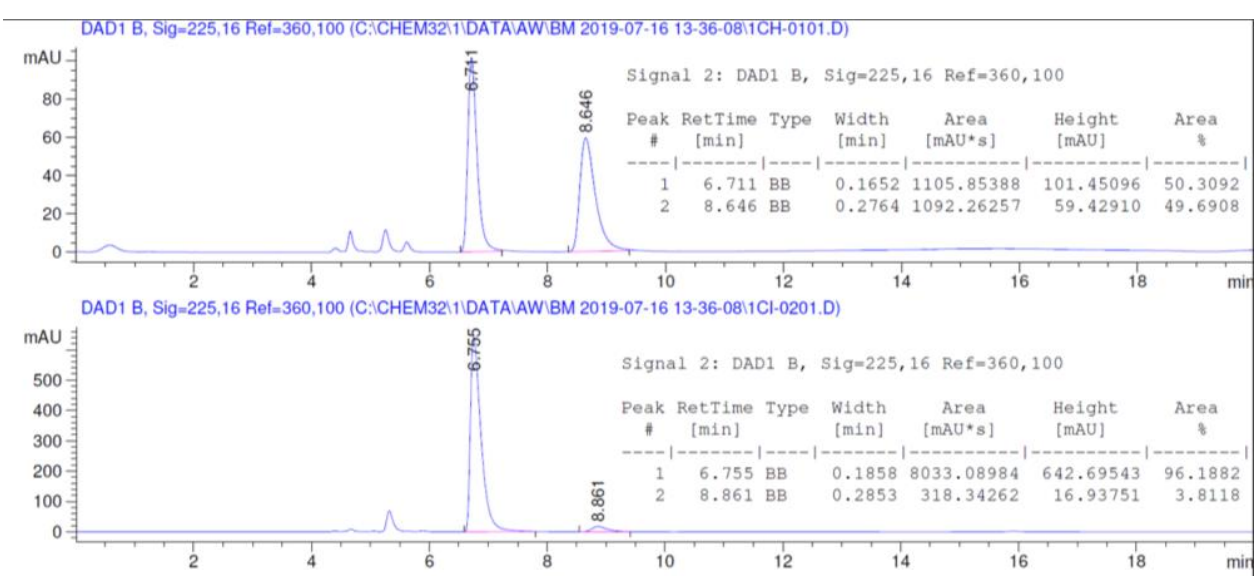
tert-butyl((1S,2S)-2-(furan-2-ylmethyl)-1,2-diphenylcyclobutoxy)dimethylsilane (3e)

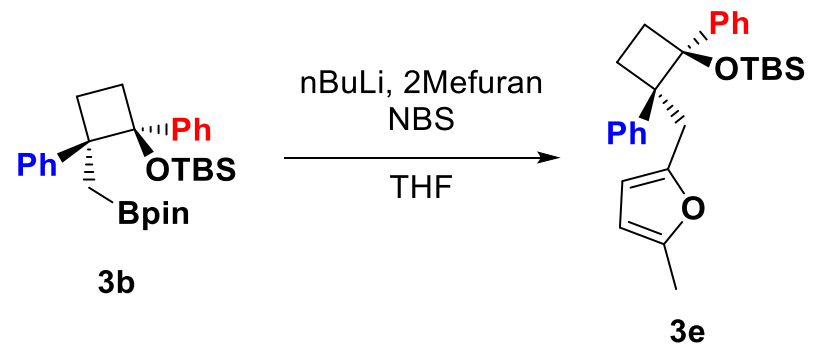

Scheme S7. Arylation procedure

To a solution of 2-methylfuran $\left(35 \mu \mathrm{L}, 0.4 \mathrm{mmol}, 2\right.$ equiv) in THF $(1 \mathrm{~mL})$ at $-78{ }^{\circ} \mathrm{C}$ was added n-butyllithium solution (2.5 M, $0.16 \mathrm{~mL}, 0.4 \mathrm{mmol}, 2$ equiv) and stirred for $20 \mathrm{~min}$ before being slowly brought up to room temperature, and stirred for an additional $30 \mathrm{~min}$. The solution was then brought back down to $-78^{\circ} \mathrm{C}$, and a solution of the boronic ester $\mathbf{3 b}$ ( $96 \mathrm{mg}, 0.2 \mathrm{mmol}, 1$ equiv) in THF ( $1 \mathrm{~mL}$ ) was added dropwise. The reaction mixture was left to stir at $-78^{\circ} \mathrm{C}$ for $1 \mathrm{~h}$, then a solution of NBS (71 mg, $0.4 \mathrm{mmol}, 2$ equiv) in THF (1 mL) was added dropwise and left to continue stirring for an additional $1 \mathrm{~h}$ at $-78{ }^{\circ} \mathrm{C}$. The reaction mixture was quenched with a $5 \%$ (w/v) solution of $\mathrm{Na}_{2} \mathrm{~S}_{2} \mathrm{O}_{3}(2 \mathrm{~mL})$, warmed to room temperature and extracted with EtOAc. Organic layers were combined, dried over $\mathrm{MgSO}_{4}$ and concentrated under vacuum. The crude product was purified by column chromatography (0-15\% DCM:pentanes) to afford the product (49. mg, 57\% yield, 96:4 er) as a colorless oil.

${ }^{1} \mathbf{H}$ NMR $\left(500 \mathrm{MHz}, \mathrm{CDCl}_{3}\right) \delta 7.57(\mathrm{dd}, J=8.3,1.3 \mathrm{~Hz}, 2 \mathrm{H}), 7.40(\mathrm{t}, J=7.7 \mathrm{~Hz}, 2 \mathrm{H}), 7.35(\mathrm{dd}$, $J=8.3,1.4 \mathrm{~Hz}, 2 \mathrm{H}), 7.33-7.28(\mathrm{~m}, 3 \mathrm{H}), 7.20-7.15(\mathrm{~m}, 1 \mathrm{H}), 5.55(\mathrm{dd}, J=3.0,1.1 \mathrm{~Hz}, 1 \mathrm{H})$, $5.11(\mathrm{~d}, J=3.0 \mathrm{~Hz}, 1 \mathrm{H}), 2.96(\mathrm{ddd}, J=12.0,8.7,6.2 \mathrm{~Hz}, 1 \mathrm{H}), 2.75(\mathrm{dddd}, J=11.6,9.3,6.2,1.1$ $\mathrm{Hz}, 1 \mathrm{H}), 2.67(\mathrm{~d}, J=15.4 \mathrm{~Hz}, 1 \mathrm{H}), 2.39$ (d, $J=15.4 \mathrm{~Hz}, 1 \mathrm{H}), 2.20(\mathrm{ddd}, J=12.0,9.3,6.7 \mathrm{~Hz}$, 1H), 2.06 (s, 3H), 2.01 (ddd, $J=11.6,8.7,6.7 \mathrm{~Hz}, 1 \mathrm{H}), 0.58$ (s, 9H), -0.24 (s, 3H), -0.45 (s, 3H).

${ }^{13} \mathbf{C ~ N M R}\left(126 \mathrm{MHz}, \mathrm{CDCl}_{3}\right) \delta 151.5,149.5,143.4,142.0,128.3,127.9,127.3,127.1,127.0$, $125.5,106.8,105.6,82.8,56.6,37.8,29.5,25.6,23.7,17.9,13.3,-3.1,-3.3$.

IR (ATR) 2965, 2933, 2858, 1609, 1586, 1506, 1452, 1256, 1138, 1006, 839, 778, 692

HRMS (ESI, M+Na) Calculated for $\mathrm{C}_{28} \mathrm{H}_{36} \mathrm{Na}_{1} \mathrm{O}_{2} \mathrm{Si}_{1} 455.2377$, found 455.2372

$\boldsymbol{\alpha}_{\boldsymbol{D}}^{\mathbf{2 0}}\left(\mathrm{c}=0.195, \mathrm{CH}_{2} \mathrm{Cl}_{2}\right) 28.4$

HPLC: IB, $100 \%$ hexane, $0.4 \mathrm{~mL} / \mathrm{min}$

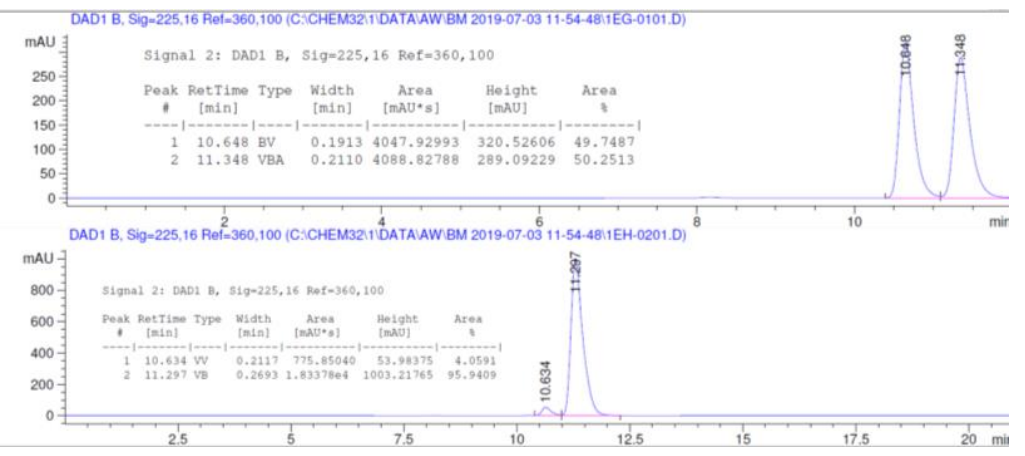


((1S,2S)-2-allyl-1,2-diphenylcyclobutoxy)(tert-butyl)dimethylsilane (3f)

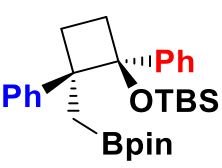

$3 b$

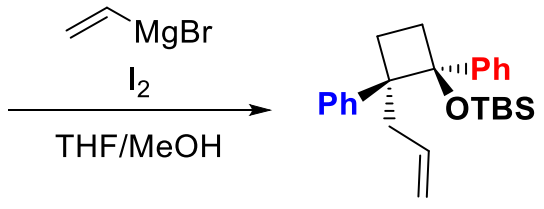

$3 \mathbf{f}$

Scheme S8. Vinylation procedure

To a solution of the boronic ester $3 \mathbf{b}(95.6 \mathrm{mg}, 0.2 \mathrm{mmol})$ in THF $(2 \mathrm{~mL})$ at $-78{ }^{\circ} \mathrm{C}$ was added a $1 \mathrm{M}$ solution of vinylmagnesium bromide in THF $(1.2 \mathrm{~mL}, 1.2 \mathrm{mmol}, 6$ equiv $)$ dropwise. The reaction mixture was left to stir at $-78{ }^{\circ} \mathrm{C}$ for $1.5 \mathrm{~h}$, then a solution of iodine $(152 \mathrm{mg}, 1.2 \mathrm{mmol}$, 6 equiv) in $\mathrm{MeOH}(1.6 \mathrm{~mL})$ was added dropwise and left to continue stirring for an additional 20 min at $-78^{\circ} \mathrm{C}$. The reaction mixture was then placed in an ice bath and allowed to warm to $0{ }^{\circ} \mathrm{C}$ and stirred for $1.5 \mathrm{~h}$. A $5 \%(\mathrm{w} / \mathrm{v})$ solution of $\mathrm{Na}_{2} \mathrm{~S}_{2} \mathrm{O}_{3}$ was added until colorless, and extracted with EtOAc (3x $20 \mathrm{~mL})$. Organic layers were combined, dried over $\mathrm{MgSO}_{4}$ and concentrated under vacuum. The crude product was purified by flash column chromatography $(100 \%$ pentanes) to afford the product (51 mg, 67\% yield, 96:4 er) as a colorless oil.

${ }^{1} \mathbf{H}$ NMR $\left(500 \mathrm{MHz}, \mathrm{CDCl}_{3}\right) \delta 7.52(\mathrm{dd}, J=8.3,1.3 \mathrm{~Hz}, 2 \mathrm{H}), 7.41-7.27(\mathrm{~m}, 7 \mathrm{H}), 7.19(\mathrm{tt}, J=$ $6.4,2.1 \mathrm{~Hz}, 1 \mathrm{H}), 5.17$ (dddd, $J=17.1,10.3,8.2,5.8 \mathrm{~Hz}, 1 \mathrm{H}), 4.79-4.68(\mathrm{~m}, 2 \mathrm{H}), 2.90$ (ddd, $J=$ $12.1,8.8,6.7 \mathrm{~Hz}, 1 \mathrm{H}), 2.70$ (dddd, $J=11.5,9.3,6.7,1.2 \mathrm{~Hz}, 1 \mathrm{H}), 2.21-2.10$ (m, 2H), $1.86-$ $1.76(\mathrm{~m}, 2 \mathrm{H}), 0.57(\mathrm{~s}, 9 \mathrm{H}),-0.24(\mathrm{~s}, 3 \mathrm{H}),-0.47(\mathrm{~s}, 3 \mathrm{H})$.

${ }^{13} \mathbf{C ~ N M R}\left(126 \mathrm{MHz}, \mathrm{CDCl}_{3}\right) \delta 143.5,142.3,135.1,128.2,127.7,127.4,127.0,125.5,116.4$, 83.0, 56.6, 43.6, 29.0, 25.6, 23.2, 17.9, -3.0, -3.4.

IR (ATR) 3061, 2958, 2929, 2858, 1648, 1602, 1502, 1445, 1260, 1138, 1003, 913, 831, 774, 696

HRMS (ESI, M+Na) Calculated for $\mathrm{C}_{25} \mathrm{H}_{34} \mathrm{Na}_{1} \mathrm{O}_{1} \mathrm{Si}_{1} 401.2271$, found 401.2268

$\boldsymbol{\alpha}_{\boldsymbol{D}}^{\mathbf{2 0}}\left(\mathrm{c}=0.270, \mathrm{CH}_{2} \mathrm{Cl}_{2}\right) 20.5$

HPLC: OD-H, $100 \%$ hexane, $0.2 \mathrm{~mL} / \mathrm{min}$

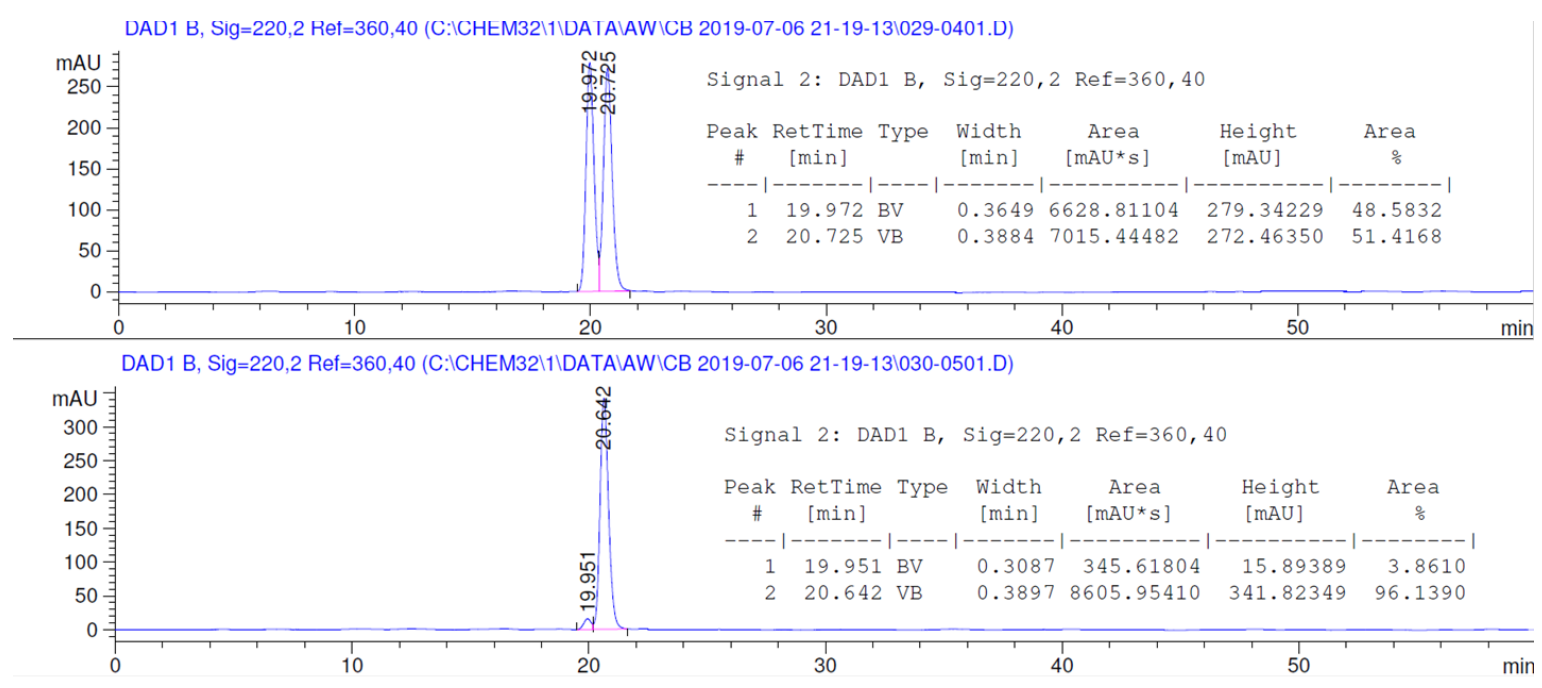




\section{Large Scale Experiment}

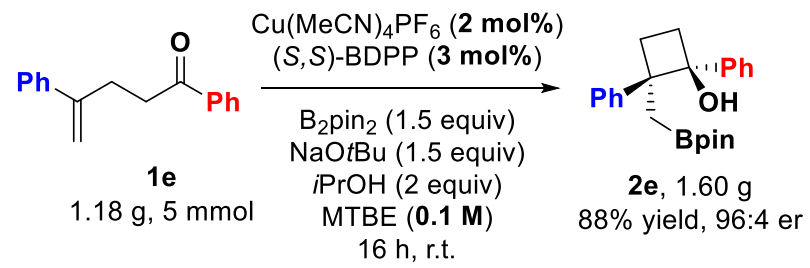

Scheme S9. Large scale experiment

To an oven dried round bottom flash equipped with a stir bar under argon was added $\mathrm{Cu}(\mathrm{MeCN})_{4} \mathrm{PF}_{6}$ (37 mg, $0.1 \mathrm{mmol}, 2 \mathrm{~mol} \%$ ), (S,S)-BDPP (66 mg, $0.15 \mathrm{mmol}, 3 \mathrm{~mol} \%$ ), and $\mathrm{NaO} t \mathrm{Bu}(721 \mathrm{mg}, 7.5 \mathrm{mmol}, 1.5$ equiv). MTBE $(12.5 \mathrm{~mL})$ was added and the catalyst solution was stirred for 5 minutes. Then a solution of $\mathrm{B}_{2} \operatorname{pin}_{2}$ (1.90 g, $7.5 \mathrm{mmol}, 1.5$ equiv) in MTBE (12.5 $\mathrm{mL})$. The suspension was stirred for 5 minutes. Then a solution of substrate $(1.18 \mathrm{~g}, 5 \mathrm{mmol})$ and $i \mathrm{PrOH}(0.76 \mathrm{~mL}, 10 \mathrm{mmol}, 2$ equiv) and MTBE $(12.5 \mathrm{~mL})$ was added. The reaction was stirred for $16 \mathrm{~h}$ at room temperature. Filtered through silica pad. The crude was purified by flash column chromatography, 0 -> 4\% ccetone/pentane. Obtained $1.60 \mathrm{~g}$ of a white solid, $88 \%$ yield, 96:4 er. 


\section{Failed substrates}<smiles>C=C(CCC(=O)c1ccccc1)c1ccc(C(F)(F)F)cc1</smiles><smiles>C=C(CCC(=O)c1ccccc1)c1ccc(Cl)cc1</smiles><smiles>C=C(CCC(=O)c1ccccc1)c1ccc(OC)cc1</smiles><smiles>C=C(CCC(=O)c1ccc(F)cc1)c1ccc(OC)cc1</smiles><smiles>C=C(C#Cc1ccccc1)CCC(=O)c1ccccc1</smiles><smiles>C=C(CC(=O)c1ccccc1)c1ccccc1</smiles><smiles>C=C(C)c1ccccc1C(C)=O</smiles><smiles>C=C(c1ccccc1)c1ccccc1C(=O)O</smiles><smiles>C=C(CCC(=NC(=O)NCCCC)c1ccccc1)c1ccccc1</smiles><smiles>C=C(CC/C=N/N=PN=O)c1ccccc1</smiles>

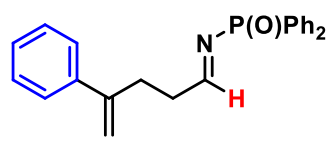<smiles>C=C(CC/C=N/[As])c1ccccc1</smiles><smiles>C=C(CCCC(=O)c1ccccc1)c1ccccc1</smiles><smiles>COC(C)=O</smiles>

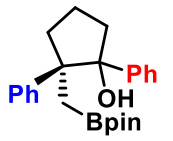

$<10 \%$ yield

Table S1. Failed substrates 


\section{X-ray data for $2 e$}

Table 1. Crystal data and structure refinement for d18188_a.

Identification code

Empirical formula

Formula weight

Temperature

Wavelength

Crystal system

Space group

Unit cell dimensions

Volume

Z

Density (calculated)

Absorption coefficient

$\mathrm{F}(000)$

Crystal size

Theta range for data collection

Index ranges

Reflections collected

Independent reflections

Completeness to theta $=67.679^{\circ}$

Absorption correction

Max. and min. transmission

Refinement method

Data / restraints / parameters

Goodness-of-fit on $\mathrm{F}^{2}$

Final $\mathrm{R}$ indices [I $>2 \operatorname{sigma}(\mathrm{I})]$

$\mathrm{R}$ indices (all data)

Absolute structure parameter

Extinction coefficient

Largest diff. peak and hole d18188_a

C23 H29 B O3

364.27

150(2) K

$1.54178 \AA$

Orthorhombic

$\mathrm{P} 2{ }_{12} 22_{1}$

$a=11.8365(3) \AA \quad a=90^{\circ}$.

$\mathrm{b}=12.8133(4) \AA \quad \mathrm{b}=90^{\circ}$.

$\mathrm{c}=13.5157(4) \AA \quad \mathrm{g}=90^{\circ}$.

2049.85(10) $\AA^{3}$

4

$1.180 \mathrm{Mg} / \mathrm{m}^{3}$

$0.593 \mathrm{~mm}^{-1}$

784

$0.240 \times 0.160 \times 0.040 \mathrm{~mm}^{3}$

4.755 to $67.686^{\circ}$.

$-14<=\mathrm{h}<=14,-15<=\mathrm{k}<=15,-15<=\mathrm{l}<=16$

38682

$3708[\mathrm{R}(\mathrm{int})=0.0327]$

$100.0 \%$

Semi-empirical from equivalents

0.7530 and 0.6949

Full-matrix least-squares on $\mathrm{F}^{2}$

3708 / 0 / 252

1.047

$\mathrm{R} 1=0.0269, \mathrm{wR} 2=0.0678$

$\mathrm{R} 1=0.0274, \mathrm{wR} 2=0.0685$

$-0.03(4)$

$\mathrm{n} / \mathrm{a}$

0.105 and -0.156 e. $\AA^{-3}$ 
Table 2. Atomic coordinates ( $\left.\mathrm{x} 10^{4}\right)$ and equivalent isotropic displacement parameters $\left(\AA^{2} \mathrm{x}\right.$ $10^{3}$ )

for d18188_a. U(eq) is defined as one third of the trace of the orthogonalized $U^{i j}$ tensor.

\begin{tabular}{|c|c|c|c|c|}
\hline & $\mathrm{x}$ & $\mathrm{y}$ & $\mathrm{z}$ & $\mathrm{U}(\mathrm{eq})$ \\
\hline \multicolumn{5}{|l|}{-} \\
\hline $\mathrm{O}(1)$ & $8861(1)$ & 2131(1) & $5331(1)$ & $27(1)$ \\
\hline $\mathrm{O}(2)$ & $6302(1)$ & 1936(1) & $1874(1)$ & $29(1)$ \\
\hline $\mathrm{O}(3)$ & $4687(1)$ & 2133(1) & $2767(1)$ & $27(1)$ \\
\hline $\mathrm{C}(1)$ & $7431(1)$ & $2109(1)$ & $4047(1)$ & $21(1)$ \\
\hline$C(2)$ & $6908(1)$ & 1137(1) & $4563(1)$ & $28(1)$ \\
\hline$C(3)$ & $6849(1)$ & $1758(1)$ & $5536(1)$ & $29(1)$ \\
\hline $\mathrm{C}(4)$ & $7755(1)$ & $2515(1)$ & $5122(1)$ & $22(1)$ \\
\hline$C(5)$ & $7679(1)$ & $3664(1)$ & $5364(1)$ & $22(1)$ \\
\hline$C(6)$ & $6726(1)$ & $4108(1)$ & $5798(1)$ & $26(1)$ \\
\hline$C(7)$ & $6713(2)$ & $5155(1)$ & $6066(1)$ & $33(1)$ \\
\hline$C(8)$ & $7636(2)$ & $5787(1)$ & $5886(1)$ & $37(1)$ \\
\hline $\mathrm{C}(9)$ & $8582(2)$ & $5357(1)$ & $5439(1)$ & $36(1)$ \\
\hline$C(10)$ & $8609(1)$ & 4307(1) & $5190(1)$ & $28(1)$ \\
\hline $\mathrm{C}(11)$ & $8385(1)$ & $1924(1)$ & $3319(1)$ & $23(1)$ \\
\hline $\mathrm{C}(12)$ & $9052(1)$ & $2750(1)$ & 2995(1) & $27(1)$ \\
\hline $\mathrm{C}(13)$ & $9911(1)$ & $2598(2)$ & $2311(1)$ & $34(1)$ \\
\hline$C(14)$ & 10104(2) & $1616(2)$ & $1922(1)$ & $38(1)$ \\
\hline $\mathrm{C}(15)$ & $9437(2)$ & $792(2)$ & $2221(1)$ & $40(1)$ \\
\hline$C(16)$ & $8591(1)$ & 941(1) & 2921(1) & $32(1)$ \\
\hline $\mathrm{C}(17)$ & $6508(1)$ & $2812(1)$ & 3591(1) & $22(1)$ \\
\hline $\mathrm{C}(18)$ & $5447(1)$ & $1324(1)$ & 1359(1) & $30(1)$ \\
\hline $\mathrm{C}(19)$ & $5608(2)$ & $1495(2)$ & $256(1)$ & $50(1)$ \\
\hline $\mathrm{C}(20)$ & $5671(2)$ & 192(2) & $1629(2)$ & $53(1)$ \\
\hline $\mathrm{C}(21)$ & $4324(1)$ & 1757(1) & 1790(1) & $27(1)$ \\
\hline $\mathrm{C}(22)$ & $3867(2)$ & 2699(2) & $1244(2)$ & $44(1)$ \\
\hline $\mathrm{C}(23)$ & $3397(2)$ & $950(2)$ & $1928(1)$ & $41(1)$ \\
\hline $\mathrm{B}(1)$ & $5834(1)$ & $2289(1)$ & $2727(1)$ & $21(1)$ \\
\hline
\end{tabular}


Table 3. Bond lengths $[\AA]$ and angles $\left[{ }^{\circ}\right]$ for d18188_a.

\begin{tabular}{|c|c|}
\hline $\mathrm{O}(1)-\mathrm{C}(4)$ & $1.4269(18)$ \\
\hline $\mathrm{O}(1)-\mathrm{H}(1 \mathrm{O})$ & $0.88(3)$ \\
\hline $\mathrm{O}(2)-\mathrm{B}(1)$ & $1.357(2)$ \\
\hline $\mathrm{O}(2)-\mathrm{C}(18)$ & $1.4564(18)$ \\
\hline $\mathrm{O}(3)-\mathrm{B}(1)$ & $1.3734(19)$ \\
\hline $\mathrm{O}(3)-\mathrm{C}(21)$ & $1.4700(17)$ \\
\hline $\mathrm{C}(1)-\mathrm{C}(11)$ & $1.515(2)$ \\
\hline $\mathrm{C}(1)-\mathrm{C}(17)$ & $1.5439(19)$ \\
\hline $\mathrm{C}(1)-\mathrm{C}(2)$ & $1.557(2)$ \\
\hline $\mathrm{C}(1)-\mathrm{C}(4)$ & $1.5905(19)$ \\
\hline$C(2)-C(3)$ & $1.538(2)$ \\
\hline $\mathrm{C}(2)-\mathrm{H}(2 \mathrm{~A})$ & 0.9900 \\
\hline $\mathrm{C}(2)-\mathrm{H}(2 \mathrm{~B})$ & 0.9900 \\
\hline$C(3)-C(4)$ & $1.551(2)$ \\
\hline $\mathrm{C}(3)-\mathrm{H}(3 \mathrm{~A})$ & 0.9900 \\
\hline $\mathrm{C}(3)-\mathrm{H}(3 \mathrm{~B})$ & 0.9900 \\
\hline$C(4)-C(5)$ & $1.511(2)$ \\
\hline$C(5)-C(6)$ & $1.393(2)$ \\
\hline $\mathrm{C}(5)-\mathrm{C}(10)$ & $1.396(2)$ \\
\hline$C(6)-C(7)$ & $1.390(2)$ \\
\hline $\mathrm{C}(6)-\mathrm{H}(6 \mathrm{~A})$ & 0.9500 \\
\hline$C(7)-C(8)$ & $1.381(3)$ \\
\hline $\mathrm{C}(7)-\mathrm{H}(7 \mathrm{~A})$ & 0.9500 \\
\hline $\mathrm{C}(8)-\mathrm{C}(9)$ & $1.387(3)$ \\
\hline $\mathrm{C}(8)-\mathrm{H}(8 \mathrm{~A})$ & 0.9500 \\
\hline $\mathrm{C}(9)-\mathrm{C}(10)$ & $1.388(2)$ \\
\hline $\mathrm{C}(9)-\mathrm{H}(9 \mathrm{~A})$ & 0.9500 \\
\hline $\mathrm{C}(10)-\mathrm{H}(10 \mathrm{~A})$ & 0.9500 \\
\hline$C(11)-C(12)$ & $1.391(2)$ \\
\hline $\mathrm{C}(11)-\mathrm{C}(16)$ & $1.392(2)$ \\
\hline $\mathrm{C}(12)-\mathrm{C}(13)$ & $1.387(2)$ \\
\hline $\mathrm{C}(12)-\mathrm{H}(12 \mathrm{~A})$ & 0.9500 \\
\hline $\mathrm{C}(13)-\mathrm{C}(14)$ & $1.383(3)$ \\
\hline $\mathrm{C}(13)-\mathrm{H}(13 \mathrm{~A})$ & 0.9500 \\
\hline
\end{tabular}




\begin{tabular}{|c|c|}
\hline$C(14)-C(15)$ & $1.380(3)$ \\
\hline $\mathrm{C}(14)-\mathrm{H}(14 \mathrm{~A})$ & 0.9500 \\
\hline$C(15)-C(16)$ & $1.391(2)$ \\
\hline $\mathrm{C}(15)-\mathrm{H}(15 \mathrm{~A})$ & 0.9500 \\
\hline $\mathrm{C}(16)-\mathrm{H}(16 \mathrm{~A})$ & 0.9500 \\
\hline $\mathrm{C}(17)-\mathrm{B}(1)$ & $1.565(2)$ \\
\hline $\mathrm{C}(17)-\mathrm{H}(17 \mathrm{~A})$ & 0.9900 \\
\hline $\mathrm{C}(17)-\mathrm{H}(17 \mathrm{~B})$ & 0.9900 \\
\hline $\mathrm{C}(18)-\mathrm{C}(20)$ & $1.519(3)$ \\
\hline $\mathrm{C}(18)-\mathrm{C}(19)$ & $1.520(2)$ \\
\hline $\mathrm{C}(18)-\mathrm{C}(21)$ & $1.554(2)$ \\
\hline C(19)-H(19A) & 0.9800 \\
\hline C(19)-H(19B) & 0.9800 \\
\hline C(19)-H(19C) & 0.9800 \\
\hline $\mathrm{C}(20)-\mathrm{H}(20 \mathrm{~A})$ & 0.9800 \\
\hline $\mathrm{C}(20)-\mathrm{H}(20 \mathrm{~B})$ & 0.9800 \\
\hline $\mathrm{C}(20)-\mathrm{H}(20 \mathrm{C})$ & 0.9800 \\
\hline$C(21)-C(22)$ & $1.515(3)$ \\
\hline $\mathrm{C}(21)-\mathrm{C}(23)$ & $1.518(2)$ \\
\hline $\mathrm{C}(22)-\mathrm{H}(22 \mathrm{~A})$ & 0.9800 \\
\hline $\mathrm{C}(22)-\mathrm{H}(22 \mathrm{~B})$ & 0.9800 \\
\hline $\mathrm{C}(22)-\mathrm{H}(22 \mathrm{C})$ & 0.9800 \\
\hline $\mathrm{C}(23)-\mathrm{H}(23 \mathrm{~A})$ & 0.9800 \\
\hline $\mathrm{C}(23)-\mathrm{H}(23 \mathrm{~B})$ & 0.9800 \\
\hline $\mathrm{C}(23)-\mathrm{H}(23 \mathrm{C})$ & 0.9800 \\
\hline $\mathrm{C}(4)-\mathrm{O}(1)-\mathrm{H}(1 \mathrm{O})$ & $107.4(16)$ \\
\hline $\mathrm{B}(1)-\mathrm{O}(2)-\mathrm{C}(18)$ & $107.60(11)$ \\
\hline $\mathrm{B}(1)-\mathrm{O}(3)-\mathrm{C}(21)$ & $107.53(12)$ \\
\hline $\mathrm{C}(11)-\mathrm{C}(1)-\mathrm{C}(17)$ & $111.10(12)$ \\
\hline $\mathrm{C}(11)-\mathrm{C}(1)-\mathrm{C}(2)$ & $117.55(12)$ \\
\hline $\mathrm{C}(17)-\mathrm{C}(1)-\mathrm{C}(2)$ & $111.32(12)$ \\
\hline $\mathrm{C}(11)-\mathrm{C}(1)-\mathrm{C}(4)$ & $117.68(12)$ \\
\hline $\mathrm{C}(17)-\mathrm{C}(1)-\mathrm{C}(4)$ & $110.10(11)$ \\
\hline $\mathrm{C}(2)-\mathrm{C}(1)-\mathrm{C}(4)$ & $87.00(11)$ \\
\hline$C(3)-C(2)-C(1)$ & $89.31(11)$ \\
\hline
\end{tabular}




\begin{tabular}{|c|c|}
\hline $\mathrm{C}(3)-\mathrm{C}(2)-\mathrm{H}(2 \mathrm{~A})$ & 113.8 \\
\hline $\mathrm{C}(1)-\mathrm{C}(2)-\mathrm{H}(2 \mathrm{~A})$ & 113.8 \\
\hline $\mathrm{C}(3)-\mathrm{C}(2)-\mathrm{H}(2 \mathrm{~B})$ & 113.8 \\
\hline $\mathrm{C}(1)-\mathrm{C}(2)-\mathrm{H}(2 \mathrm{~B})$ & 113.8 \\
\hline $\mathrm{H}(2 \mathrm{~A})-\mathrm{C}(2)-\mathrm{H}(2 \mathrm{~B})$ & 111.0 \\
\hline $\mathrm{C}(2)-\mathrm{C}(3)-\mathrm{C}(4)$ & $89.06(12)$ \\
\hline $\mathrm{C}(2)-\mathrm{C}(3)-\mathrm{H}(3 \mathrm{~A})$ & 113.8 \\
\hline $\mathrm{C}(4)-\mathrm{C}(3)-\mathrm{H}(3 \mathrm{~A})$ & 113.8 \\
\hline $\mathrm{C}(2)-\mathrm{C}(3)-\mathrm{H}(3 \mathrm{~B})$ & 113.8 \\
\hline $\mathrm{C}(4)-\mathrm{C}(3)-\mathrm{H}(3 \mathrm{~B})$ & 113.8 \\
\hline $\mathrm{H}(3 \mathrm{~A})-\mathrm{C}(3)-\mathrm{H}(3 \mathrm{~B})$ & 111.0 \\
\hline $\mathrm{O}(1)-\mathrm{C}(4)-\mathrm{C}(5)$ & $110.36(12)$ \\
\hline $\mathrm{O}(1)-\mathrm{C}(4)-\mathrm{C}(3)$ & $110.32(12)$ \\
\hline$C(5)-C(4)-C(3)$ & $119.35(13)$ \\
\hline $\mathrm{O}(1)-\mathrm{C}(4)-\mathrm{C}(1)$ & $106.82(11)$ \\
\hline$C(5)-C(4)-C(1)$ & $120.14(12)$ \\
\hline $\mathrm{C}(3)-\mathrm{C}(4)-\mathrm{C}(1)$ & $87.62(11)$ \\
\hline$C(6)-C(5)-C(10)$ & $117.98(14)$ \\
\hline$C(6)-C(5)-C(4)$ & $122.52(14)$ \\
\hline$C(10)-C(5)-C(4)$ & $119.44(14)$ \\
\hline$C(7)-C(6)-C(5)$ & $120.85(16)$ \\
\hline$C(7)-C(6)-H(6 A)$ & 119.6 \\
\hline $\mathrm{C}(5)-\mathrm{C}(6)-\mathrm{H}(6 \mathrm{~A})$ & 119.6 \\
\hline$C(8)-C(7)-C(6)$ & $120.75(17)$ \\
\hline $\mathrm{C}(8)-\mathrm{C}(7)-\mathrm{H}(7 \mathrm{~A})$ & 119.6 \\
\hline $\mathrm{C}(6)-\mathrm{C}(7)-\mathrm{H}(7 \mathrm{~A})$ & 119.6 \\
\hline$C(7)-C(8)-C(9)$ & $118.88(15)$ \\
\hline $\mathrm{C}(7)-\mathrm{C}(8)-\mathrm{H}(8 \mathrm{~A})$ & 120.6 \\
\hline $\mathrm{C}(9)-\mathrm{C}(8)-\mathrm{H}(8 \mathrm{~A})$ & 120.6 \\
\hline$C(8)-C(9)-C(10)$ & $120.64(17)$ \\
\hline $\mathrm{C}(8)-\mathrm{C}(9)-\mathrm{H}(9 \mathrm{~A})$ & 119.7 \\
\hline $\mathrm{C}(10)-\mathrm{C}(9)-\mathrm{H}(9 \mathrm{~A})$ & 119.7 \\
\hline$C(9)-C(10)-C(5)$ & $120.87(16)$ \\
\hline $\mathrm{C}(9)-\mathrm{C}(10)-\mathrm{H}(10 \mathrm{~A})$ & 119.6 \\
\hline $\mathrm{C}(5)-\mathrm{C}(10)-\mathrm{H}(10 \mathrm{~A})$ & 119.6 \\
\hline $\mathrm{C}(12)-\mathrm{C}(11)-\mathrm{C}(16)$ & $117.83(14)$ \\
\hline
\end{tabular}




\begin{tabular}{|c|c|}
\hline$C(12)-C(11)-C(1)$ & $120.58(13)$ \\
\hline $\mathrm{C}(16)-\mathrm{C}(11)-\mathrm{C}(1)$ & $121.53(14)$ \\
\hline$C(13)-C(12)-C(11)$ & $121.31(15)$ \\
\hline $\mathrm{C}(13)-\mathrm{C}(12)-\mathrm{H}(12 \mathrm{~A})$ & 119.3 \\
\hline $\mathrm{C}(11)-\mathrm{C}(12)-\mathrm{H}(12 \mathrm{~A})$ & 119.3 \\
\hline $\mathrm{C}(14)-\mathrm{C}(13)-\mathrm{C}(12)$ & $120.15(17)$ \\
\hline $\mathrm{C}(14)-\mathrm{C}(13)-\mathrm{H}(13 \mathrm{~A})$ & 119.9 \\
\hline $\mathrm{C}(12)-\mathrm{C}(13)-\mathrm{H}(13 \mathrm{~A})$ & 119.9 \\
\hline$C(15)-C(14)-C(13)$ & $119.36(15)$ \\
\hline $\mathrm{C}(15)-\mathrm{C}(14)-\mathrm{H}(14 \mathrm{~A})$ & 120.3 \\
\hline $\mathrm{C}(13)-\mathrm{C}(14)-\mathrm{H}(14 \mathrm{~A})$ & 120.3 \\
\hline$C(14)-C(15)-C(16)$ & $120.41(16)$ \\
\hline $\mathrm{C}(14)-\mathrm{C}(15)-\mathrm{H}(15 \mathrm{~A})$ & 119.8 \\
\hline $\mathrm{C}(16)-\mathrm{C}(15)-\mathrm{H}(15 \mathrm{~A})$ & 119.8 \\
\hline$C(15)-C(16)-C(11)$ & $120.92(17)$ \\
\hline $\mathrm{C}(15)-\mathrm{C}(16)-\mathrm{H}(16 \mathrm{~A})$ & 119.5 \\
\hline $\mathrm{C}(11)-\mathrm{C}(16)-\mathrm{H}(16 \mathrm{~A})$ & 119.5 \\
\hline $\mathrm{C}(1)-\mathrm{C}(17)-\mathrm{B}(1)$ & $114.10(12)$ \\
\hline $\mathrm{C}(1)-\mathrm{C}(17)-\mathrm{H}(17 \mathrm{~A})$ & 108.7 \\
\hline $\mathrm{B}(1)-\mathrm{C}(17)-\mathrm{H}(17 \mathrm{~A})$ & 108.7 \\
\hline $\mathrm{C}(1)-\mathrm{C}(17)-\mathrm{H}(17 \mathrm{~B})$ & 108.7 \\
\hline $\mathrm{B}(1)-\mathrm{C}(17)-\mathrm{H}(17 \mathrm{~B})$ & 108.7 \\
\hline $\mathrm{H}(17 \mathrm{~A})-\mathrm{C}(17)-\mathrm{H}(17 \mathrm{~B})$ & 107.6 \\
\hline $\mathrm{O}(2)-\mathrm{C}(18)-\mathrm{C}(20)$ & $106.18(14)$ \\
\hline $\mathrm{O}(2)-\mathrm{C}(18)-\mathrm{C}(19)$ & $107.69(13)$ \\
\hline$C(20)-C(18)-C(19)$ & $110.61(17)$ \\
\hline $\mathrm{O}(2)-\mathrm{C}(18)-\mathrm{C}(21)$ & $102.87(12)$ \\
\hline$C(20)-C(18)-C(21)$ & $113.56(15)$ \\
\hline $\mathrm{C}(19)-\mathrm{C}(18)-\mathrm{C}(21)$ & $115.06(15)$ \\
\hline $\mathrm{C}(18)-\mathrm{C}(19)-\mathrm{H}(19 \mathrm{~A})$ & 109.5 \\
\hline $\mathrm{C}(18)-\mathrm{C}(19)-\mathrm{H}(19 \mathrm{~B})$ & 109.5 \\
\hline $\mathrm{H}(19 \mathrm{~A})-\mathrm{C}(19)-\mathrm{H}(19 \mathrm{~B})$ & 109.5 \\
\hline $\mathrm{C}(18)-\mathrm{C}(19)-\mathrm{H}(19 \mathrm{C})$ & 109.5 \\
\hline $\mathrm{H}(19 \mathrm{~A})-\mathrm{C}(19)-\mathrm{H}(19 \mathrm{C})$ & 109.5 \\
\hline H(19B)-C(19)-H(19C) & 109.5 \\
\hline $\mathrm{C}(18)-\mathrm{C}(20)-\mathrm{H}(20 \mathrm{~A})$ & 109.5 \\
\hline
\end{tabular}




\begin{tabular}{|c|c|}
\hline $\mathrm{C}(18)-\mathrm{C}(20)-\mathrm{H}(20 \mathrm{~B})$ & 109.5 \\
\hline $\mathrm{H}(20 \mathrm{~A})-\mathrm{C}(20)-\mathrm{H}(20 \mathrm{~B})$ & 109.5 \\
\hline $\mathrm{C}(18)-\mathrm{C}(20)-\mathrm{H}(20 \mathrm{C})$ & 109.5 \\
\hline $\mathrm{H}(20 \mathrm{~A})-\mathrm{C}(20)-\mathrm{H}(20 \mathrm{C})$ & 109.5 \\
\hline $\mathrm{H}(20 \mathrm{~B})-\mathrm{C}(20)-\mathrm{H}(20 \mathrm{C})$ & 109.5 \\
\hline $\mathrm{O}(3)-\mathrm{C}(21)-\mathrm{C}(22)$ & $106.27(13)$ \\
\hline $\mathrm{O}(3)-\mathrm{C}(21)-\mathrm{C}(23)$ & $108.93(13)$ \\
\hline $\mathrm{C}(22)-\mathrm{C}(21)-\mathrm{C}(23)$ & $110.14(15)$ \\
\hline $\mathrm{O}(3)-\mathrm{C}(21)-\mathrm{C}(18)$ & $101.76(11)$ \\
\hline$C(22)-C(21)-C(18)$ & $114.00(14)$ \\
\hline $\mathrm{C}(23)-\mathrm{C}(21)-\mathrm{C}(18)$ & $114.95(15)$ \\
\hline $\mathrm{C}(21)-\mathrm{C}(22)-\mathrm{H}(22 \mathrm{~A})$ & 109.5 \\
\hline $\mathrm{C}(21)-\mathrm{C}(22)-\mathrm{H}(22 \mathrm{~B})$ & 109.5 \\
\hline $\mathrm{H}(22 \mathrm{~A})-\mathrm{C}(22)-\mathrm{H}(22 \mathrm{~B})$ & 109.5 \\
\hline $\mathrm{C}(21)-\mathrm{C}(22)-\mathrm{H}(22 \mathrm{C})$ & 109.5 \\
\hline $\mathrm{H}(22 \mathrm{~A})-\mathrm{C}(22)-\mathrm{H}(22 \mathrm{C})$ & 109.5 \\
\hline $\mathrm{H}(22 \mathrm{~B})-\mathrm{C}(22)-\mathrm{H}(22 \mathrm{C})$ & 109.5 \\
\hline $\mathrm{C}(21)-\mathrm{C}(23)-\mathrm{H}(23 \mathrm{~A})$ & 109.5 \\
\hline $\mathrm{C}(21)-\mathrm{C}(23)-\mathrm{H}(23 \mathrm{~B})$ & 109.5 \\
\hline $\mathrm{H}(23 \mathrm{~A})-\mathrm{C}(23)-\mathrm{H}(23 \mathrm{~B})$ & 109.5 \\
\hline $\mathrm{C}(21)-\mathrm{C}(23)-\mathrm{H}(23 \mathrm{C})$ & 109.5 \\
\hline $\mathrm{H}(23 \mathrm{~A})-\mathrm{C}(23)-\mathrm{H}(23 \mathrm{C})$ & 109.5 \\
\hline $\mathrm{H}(23 \mathrm{~B})-\mathrm{C}(23)-\mathrm{H}(23 \mathrm{C})$ & 109.5 \\
\hline $\mathrm{O}(2)-\mathrm{B}(1)-\mathrm{O}(3)$ & $112.85(13)$ \\
\hline $\mathrm{O}(2)-\mathrm{B}(1)-\mathrm{C}(17)$ & $124.73(13)$ \\
\hline $\mathrm{O}(3)-\mathrm{B}(1)-\mathrm{C}(17)$ & $122.42(14)$ \\
\hline
\end{tabular}

Symmetry transformations used to generate equivalent atoms: 
Table 4. Anisotropic displacement parameters $\left(\AA^{2} \times 10^{3}\right)$ for d18188_a. The anisotropic displacement factor exponent takes the form: $-2 p^{2}\left[h^{2} a^{* 2} U^{11}+\ldots+2 h k a^{*} b^{*} U^{12}\right]$

\begin{tabular}{|c|c|c|c|c|c|c|}
\hline & $\mathrm{U}^{11}$ & $\mathrm{U}^{22}$ & $\mathrm{U}^{33}$ & $\mathrm{U}^{23}$ & $\mathrm{U}^{13}$ & $\mathrm{U}^{12}$ \\
\hline $\mathrm{O}(1)$ & $25(1)$ & $33(1)$ & $24(1)$ & $-3(1)$ & $-6(1)$ & $9(1)$ \\
\hline $\mathrm{O}(2)$ & $23(1)$ & $39(1)$ & $26(1)$ & $-10(1)$ & $-1(1)$ & $-5(1)$ \\
\hline $\mathrm{O}(3)$ & $22(1)$ & $39(1)$ & $20(1)$ & $-7(1)$ & $-1(1)$ & $-4(1)$ \\
\hline $\mathrm{C}(1)$ & $20(1)$ & $21(1)$ & $22(1)$ & $-3(1)$ & $-2(1)$ & $1(1)$ \\
\hline $\mathrm{C}(2)$ & $31(1)$ & $20(1)$ & $32(1)$ & $-1(1)$ & 1(1) & $-2(1)$ \\
\hline $\mathrm{C}(3)$ & $33(1)$ & $25(1)$ & $28(1)$ & $2(1)$ & $4(1)$ & $-1(1)$ \\
\hline $\mathrm{C}(4)$ & $20(1)$ & $24(1)$ & 21(1) & $0(1)$ & $-1(1)$ & $4(1)$ \\
\hline $\mathrm{C}(5)$ & $24(1)$ & $24(1)$ & $17(1)$ & $0(1)$ & $-5(1)$ & $1(1)$ \\
\hline $\mathrm{C}(6)$ & $27(1)$ & $29(1)$ & $23(1)$ & $-2(1)$ & $-4(1)$ & $4(1)$ \\
\hline $\mathrm{C}(7)$ & $43(1)$ & $31(1)$ & $25(1)$ & $-5(1)$ & $-9(1)$ & $13(1)$ \\
\hline $\mathrm{C}(8)$ & $60(1)$ & $22(1)$ & $29(1)$ & $-3(1)$ & $-18(1)$ & $3(1)$ \\
\hline $\mathrm{C}(9)$ & $46(1)$ & $31(1)$ & $30(1)$ & $3(1)$ & $-11(1)$ & $-13(1)$ \\
\hline$C(10)$ & $28(1)$ & $33(1)$ & $23(1)$ & $-1(1)$ & $-4(1)$ & $-4(1)$ \\
\hline$C(11)$ & $21(1)$ & $28(1)$ & $20(1)$ & $-2(1)$ & $-5(1)$ & $6(1)$ \\
\hline$C(12)$ & $28(1)$ & $32(1)$ & $22(1)$ & $-2(1)$ & $-1(1)$ & $3(1)$ \\
\hline$C(13)$ & $29(1)$ & $50(1)$ & $24(1)$ & $4(1)$ & $1(1)$ & $3(1)$ \\
\hline$C(14)$ & $32(1)$ & $60(1)$ & $23(1)$ & $-3(1)$ & $3(1)$ & $17(1)$ \\
\hline$C(15)$ & $42(1)$ & $43(1)$ & $34(1)$ & $-11(1)$ & 1(1) & $18(1)$ \\
\hline$C(16)$ & $34(1)$ & $30(1)$ & $33(1)$ & $-6(1)$ & $-1(1)$ & $7(1)$ \\
\hline$C(17)$ & $19(1)$ & $23(1)$ & $24(1)$ & $-4(1)$ & $-2(1)$ & $2(1)$ \\
\hline$C(18)$ & $30(1)$ & $34(1)$ & $25(1)$ & $-9(1)$ & $-1(1)$ & $-8(1)$ \\
\hline$C(19)$ & $45(1)$ & $79(2)$ & $25(1)$ & $-16(1)$ & $6(1)$ & $-19(1)$ \\
\hline$C(20)$ & $62(1)$ & $32(1)$ & $66(1)$ & $-18(1)$ & $-7(1)$ & $3(1)$ \\
\hline$C(21)$ & $25(1)$ & $36(1)$ & $18(1)$ & $-3(1)$ & $-4(1)$ & $-9(1)$ \\
\hline $\mathrm{C}(22)$ & $40(1)$ & $50(1)$ & $41(1)$ & $5(1)$ & $-13(1)$ & $0(1)$ \\
\hline$C(23)$ & $36(1)$ & $58(1)$ & $28(1)$ & $-2(1)$ & $-2(1)$ & $-24(1)$ \\
\hline $\mathrm{B}(1)$ & $22(1)$ & 19(1) & $23(1)$ & $0(1)$ & $-1(1)$ & 1(1) \\
\hline
\end{tabular}


Table 5. Hydrogen coordinates ( $\left.\times 10^{4}\right)$ and isotropic displacement parameters $\left(\AA^{2} \times 10^{3}\right)$ for d18188_a.

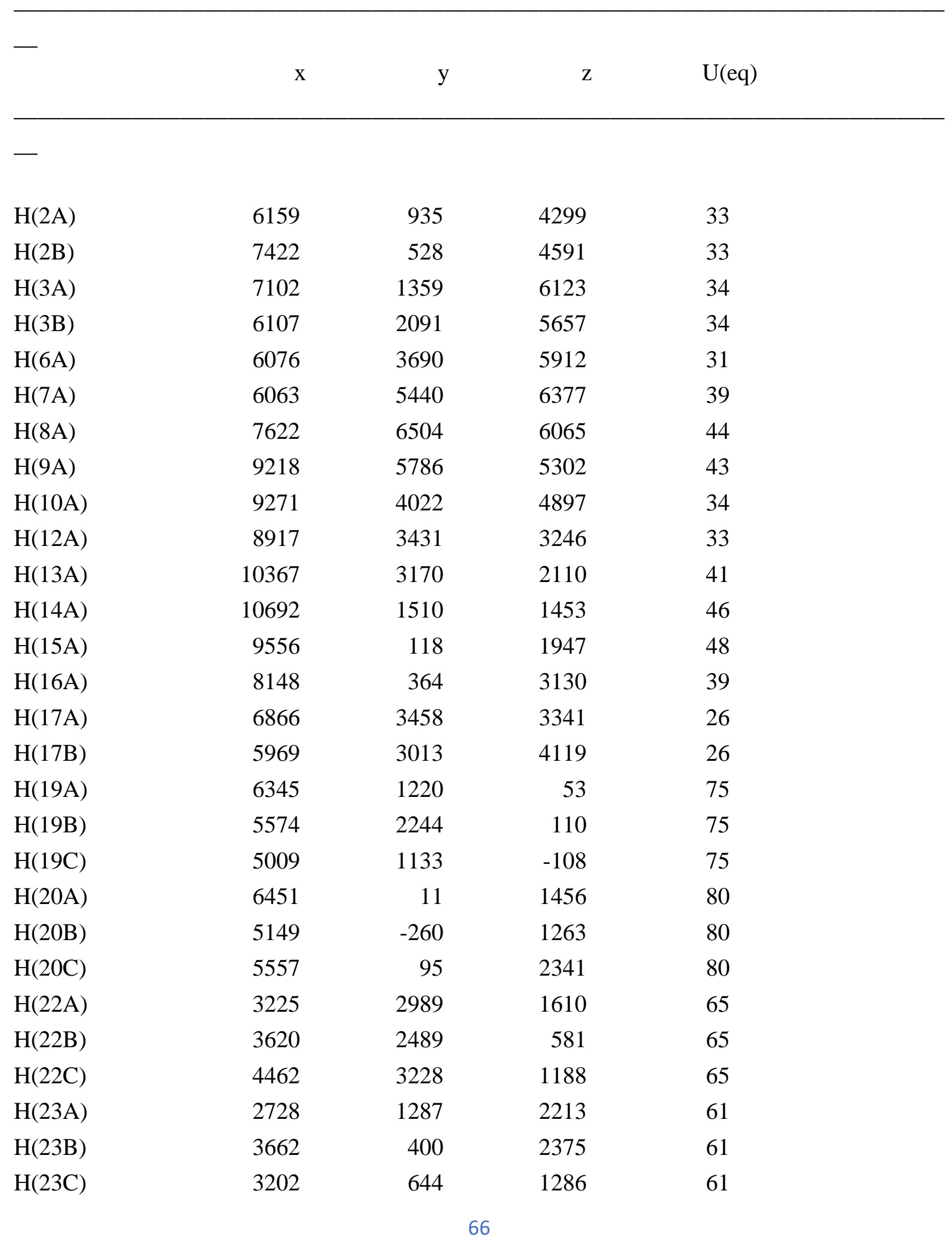


$\mathrm{H}(1 \mathrm{O})$

9040(20) 2332(18)

5935(19)

$52(6)$

Table 6. Torsion angles $\left[{ }^{\circ}\right]$ for d18188_a.

\begin{tabular}{lc}
\hline $\mathrm{C}(11)-\mathrm{C}(1)-\mathrm{C}(2)-\mathrm{C}(3)$ & $139.57(13)$ \\
$\mathrm{C}(17)-\mathrm{C}(1)-\mathrm{C}(2)-\mathrm{C}(3)$ & $-90.68(13)$ \\
$\mathrm{C}(4)-\mathrm{C}(1)-\mathrm{C}(2)-\mathrm{C}(3)$ & $19.74(11)$ \\
$\mathrm{C}(1)-\mathrm{C}(2)-\mathrm{C}(3)-\mathrm{C}(4)$ & $-20.24(11)$ \\
$\mathrm{C}(2)-\mathrm{C}(3)-\mathrm{C}(4)-\mathrm{O}(1)$ & $-87.25(13)$ \\
$\mathrm{C}(2)-\mathrm{C}(3)-\mathrm{C}(4)-\mathrm{C}(5)$ & $143.40(14)$ \\
$\mathrm{C}(2)-\mathrm{C}(3)-\mathrm{C}(4)-\mathrm{C}(1)$ & $19.81(11)$ \\
$\mathrm{C}(11)-\mathrm{C}(1)-\mathrm{C}(4)-\mathrm{O}(1)$ & $-28.78(17)$ \\
$\mathrm{C}(17)-\mathrm{C}(1)-\mathrm{C}(4)-\mathrm{O}(1)$ & $-157.45(12)$ \\
$\mathrm{C}(2)-\mathrm{C}(1)-\mathrm{C}(4)-\mathrm{O}(1)$ & $90.93(12)$ \\
$\mathrm{C}(11)-\mathrm{C}(1)-\mathrm{C}(4)-\mathrm{C}(5)$ & $97.80(16)$ \\
$\mathrm{C}(17)-\mathrm{C}(1)-\mathrm{C}(4)-\mathrm{C}(5)$ & $-30.87(18)$ \\
$\mathrm{C}(2)-\mathrm{C}(1)-\mathrm{C}(4)-\mathrm{C}(5)$ & $-142.49(14)$ \\
$\mathrm{C}(11)-\mathrm{C}(1)-\mathrm{C}(4)-\mathrm{C}(3)$ & $-139.30(14)$ \\
$\mathrm{C}(17)-\mathrm{C}(1)-\mathrm{C}(4)-\mathrm{C}(3)$ & $92.03(13)$ \\
$\mathrm{C}(2)-\mathrm{C}(1)-\mathrm{C}(4)-\mathrm{C}(3)$ & $-19.59(11)$ \\
$\mathrm{O}(1)-\mathrm{C}(4)-\mathrm{C}(5)-\mathrm{C}(6)$ & $-140.85(14)$ \\
$\mathrm{C}(3)-\mathrm{C}(4)-\mathrm{C}(5)-\mathrm{C}(6)$ & $-11.5(2)$ \\
$\mathrm{C}(1)-\mathrm{C}(4)-\mathrm{C}(5)-\mathrm{C}(6)$ & $94.23(17)$ \\
$\mathrm{O}(1)-\mathrm{C}(4)-\mathrm{C}(5)-\mathrm{C}(10)$ & $36.23(18)$ \\
$\mathrm{C}(3)-\mathrm{C}(4)-\mathrm{C}(5)-\mathrm{C}(10)$ & $165.56(14)$ \\
$\mathrm{C}(1)-\mathrm{C}(4)-\mathrm{C}(5)-\mathrm{C}(10)$ & $-88.68(17)$ \\
$\mathrm{C}(10)-\mathrm{C}(5)-\mathrm{C}(6)-\mathrm{C}(7)$ & $-1.2(2)$ \\
$\mathrm{C}(4)-\mathrm{C}(5)-\mathrm{C}(6)-\mathrm{C}(7)$ & $175.93(14)$ \\
$\mathrm{C}(5)-\mathrm{C}(6)-\mathrm{C}(7)-\mathrm{C}(8)$ & $1.6(2)$ \\
$\mathrm{C}(6)-\mathrm{C}(7)-\mathrm{C}(8)-\mathrm{C}(9)$ & $-0.5(2)$ \\
$\mathrm{C}(7)-\mathrm{C}(8)-\mathrm{C}(9)-\mathrm{C}(10)$ & $-0.9(2)$ \\
$\mathrm{C}(8)-\mathrm{C}(9)-\mathrm{C}(10)-\mathrm{C}(5)$ & $1.3(2)$ \\
$\mathrm{C}(6)-\mathrm{C}(5)-\mathrm{C}(10)-\mathrm{C}(9)$ & $-0.3(2)$ \\
$\mathrm{C}(4)-\mathrm{C}(5)-\mathrm{C}(10)-\mathrm{C}(9)$ & $-177.49(14)$ \\
& \\
& \\
& \\
&
\end{tabular}




\begin{tabular}{|c|c|}
\hline $\mathrm{C}(17)-\mathrm{C}(1)-\mathrm{C}(11)-\mathrm{C}(12)$ & $64.17(17)$ \\
\hline$C(2)-C(1)-C(11)-C(12)$ & $-165.98(14)$ \\
\hline$C(4)-C(1)-C(11)-C(12)$ & $-64.03(18)$ \\
\hline $\mathrm{C}(17)-\mathrm{C}(1)-\mathrm{C}(11)-\mathrm{C}(16)$ & $-112.92(16)$ \\
\hline$C(2)-C(1)-C(11)-C(16)$ & $16.9(2)$ \\
\hline$C(4)-C(1)-C(11)-C(16)$ & $118.88(15)$ \\
\hline$C(16)-C(11)-C(12)-C(13)$ & $-1.2(2)$ \\
\hline$C(1)-C(11)-C(12)-C(13)$ & $-178.43(14)$ \\
\hline $\mathrm{C}(11)-\mathrm{C}(12)-\mathrm{C}(13)-\mathrm{C}(14)$ & $1.3(2)$ \\
\hline$C(12)-C(13)-C(14)-C(15)$ & $0.0(3)$ \\
\hline$C(13)-C(14)-C(15)-C(16)$ & $-1.2(3)$ \\
\hline$C(14)-C(15)-C(16)-C(11)$ & $1.3(3)$ \\
\hline$C(12)-C(11)-C(16)-C(15)$ & $0.0(2)$ \\
\hline$C(1)-C(11)-C(16)-C(15)$ & $177.12(15)$ \\
\hline $\mathrm{C}(11)-\mathrm{C}(1)-\mathrm{C}(17)-\mathrm{B}(1)$ & $68.01(16)$ \\
\hline $\mathrm{C}(2)-\mathrm{C}(1)-\mathrm{C}(17)-\mathrm{B}(1)$ & $-65.05(16)$ \\
\hline $\mathrm{C}(4)-\mathrm{C}(1)-\mathrm{C}(17)-\mathrm{B}(1)$ & $-159.81(12)$ \\
\hline $\mathrm{B}(1)-\mathrm{O}(2)-\mathrm{C}(18)-\mathrm{C}(20)$ & $-96.33(17)$ \\
\hline $\mathrm{B}(1)-\mathrm{O}(2)-\mathrm{C}(18)-\mathrm{C}(19)$ & $145.16(16)$ \\
\hline $\mathrm{B}(1)-\mathrm{O}(2)-\mathrm{C}(18)-\mathrm{C}(21)$ & $23.22(16)$ \\
\hline $\mathrm{B}(1)-\mathrm{O}(3)-\mathrm{C}(21)-\mathrm{C}(22)$ & $-98.38(15)$ \\
\hline $\mathrm{B}(1)-\mathrm{O}(3)-\mathrm{C}(21)-\mathrm{C}(23)$ & $142.99(15)$ \\
\hline $\mathrm{B}(1)-\mathrm{O}(3)-\mathrm{C}(21)-\mathrm{C}(18)$ & $21.20(16)$ \\
\hline $\mathrm{O}(2)-\mathrm{C}(18)-\mathrm{C}(21)-\mathrm{O}(3)$ & $-26.49(15)$ \\
\hline $\mathrm{C}(20)-\mathrm{C}(18)-\mathrm{C}(21)-\mathrm{O}(3)$ & $87.81(16)$ \\
\hline $\mathrm{C}(19)-\mathrm{C}(18)-\mathrm{C}(21)-\mathrm{O}(3)$ & $-143.31(15)$ \\
\hline $\mathrm{O}(2)-\mathrm{C}(18)-\mathrm{C}(21)-\mathrm{C}(22)$ & $87.46(16)$ \\
\hline $\mathrm{C}(20)-\mathrm{C}(18)-\mathrm{C}(21)-\mathrm{C}(22)$ & $-158.24(15)$ \\
\hline $\mathrm{C}(19)-\mathrm{C}(18)-\mathrm{C}(21)-\mathrm{C}(22)$ & $-29.4(2)$ \\
\hline $\mathrm{O}(2)-\mathrm{C}(18)-\mathrm{C}(21)-\mathrm{C}(23)$ & $-144.02(14)$ \\
\hline $\mathrm{C}(20)-\mathrm{C}(18)-\mathrm{C}(21)-\mathrm{C}(23)$ & $-29.7(2)$ \\
\hline $\mathrm{C}(19)-\mathrm{C}(18)-\mathrm{C}(21)-\mathrm{C}(23)$ & $99.16(18)$ \\
\hline $\mathrm{C}(18)-\mathrm{O}(2)-\mathrm{B}(1)-\mathrm{O}(3)$ & $-10.73(18)$ \\
\hline $\mathrm{C}(18)-\mathrm{O}(2)-\mathrm{B}(1)-\mathrm{C}(17)$ & $168.77(14)$ \\
\hline $\mathrm{C}(21)-\mathrm{O}(3)-\mathrm{B}(1)-\mathrm{O}(2)$ & $-7.73(18)$ \\
\hline $\mathrm{C}(21)-\mathrm{O}(3)-\mathrm{B}(1)-\mathrm{C}(17)$ & $172.76(13)$ \\
\hline
\end{tabular}


$\mathrm{C}(1)-\mathrm{C}(17)-\mathrm{B}(1)-\mathrm{O}(2)$

$-59.8(2)$

$\mathrm{C}(1)-\mathrm{C}(17)-\mathrm{B}(1)-\mathrm{O}(3)$

$119.62(15)$

Symmetry transformations used to generate equivalent atoms:

Table 7. Hydrogen bonds for d18188_a [ $\left[\AA\right.$ and $\left.{ }^{\circ}\right]$.

\begin{tabular}{lllll}
\hline D-H...A & d(D-H) & d(H...A $)$ & d(D...A $)$ & $<($ DHA $)$
\end{tabular}

$\mathrm{O}(1)-\mathrm{H}(1 \mathrm{O}) \ldots \mathrm{O}(3) \# 1 \quad 0.88(3) \quad 2.03(3) \quad 2.9071(15) \quad 171(2)$

Symmetry transformations used to generate equivalent atoms:

$\# 1 \mathrm{x}+1 / 2,-y+1 / 2,-z+1$

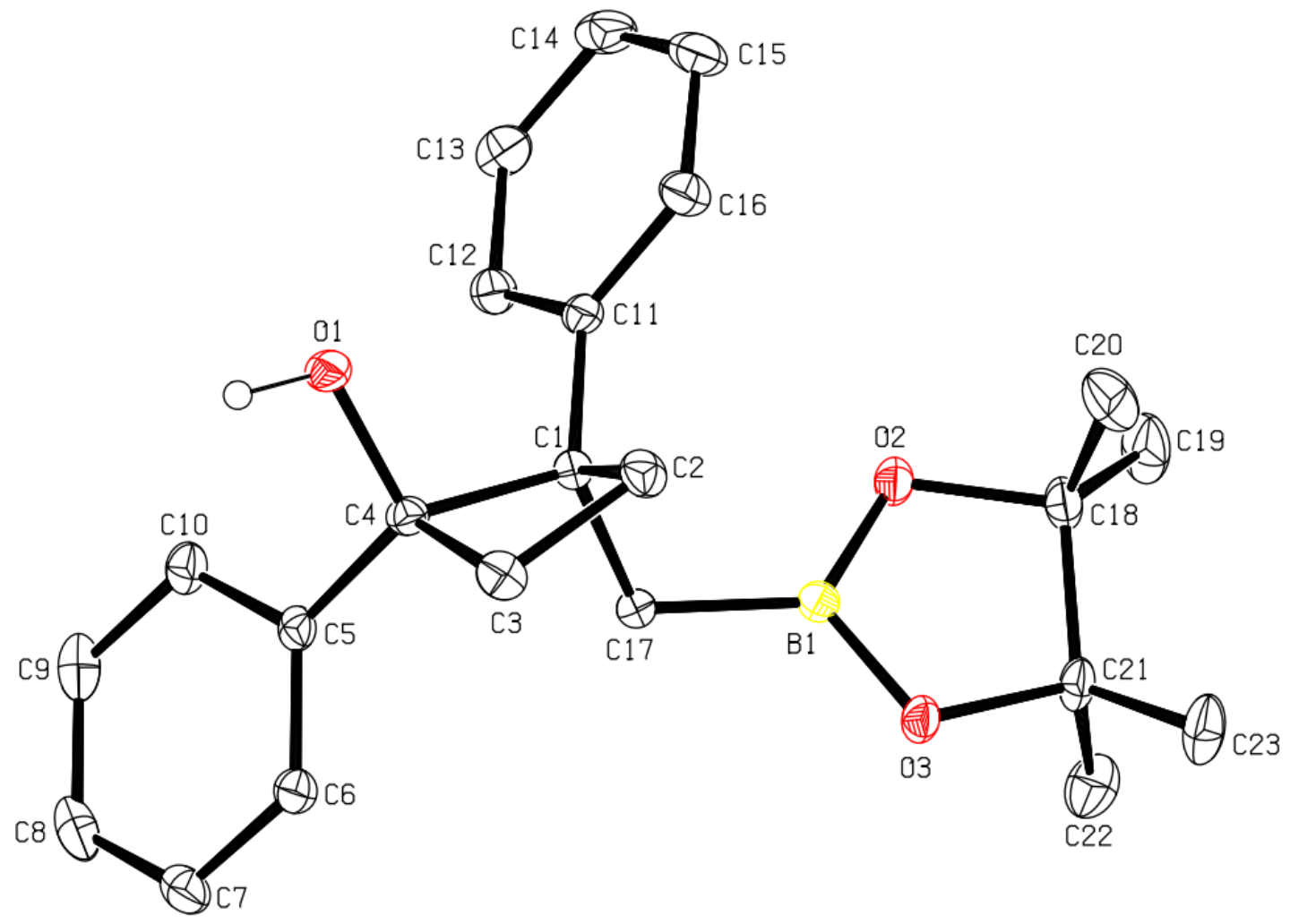

Figure S1. X-ray structure of product 2 

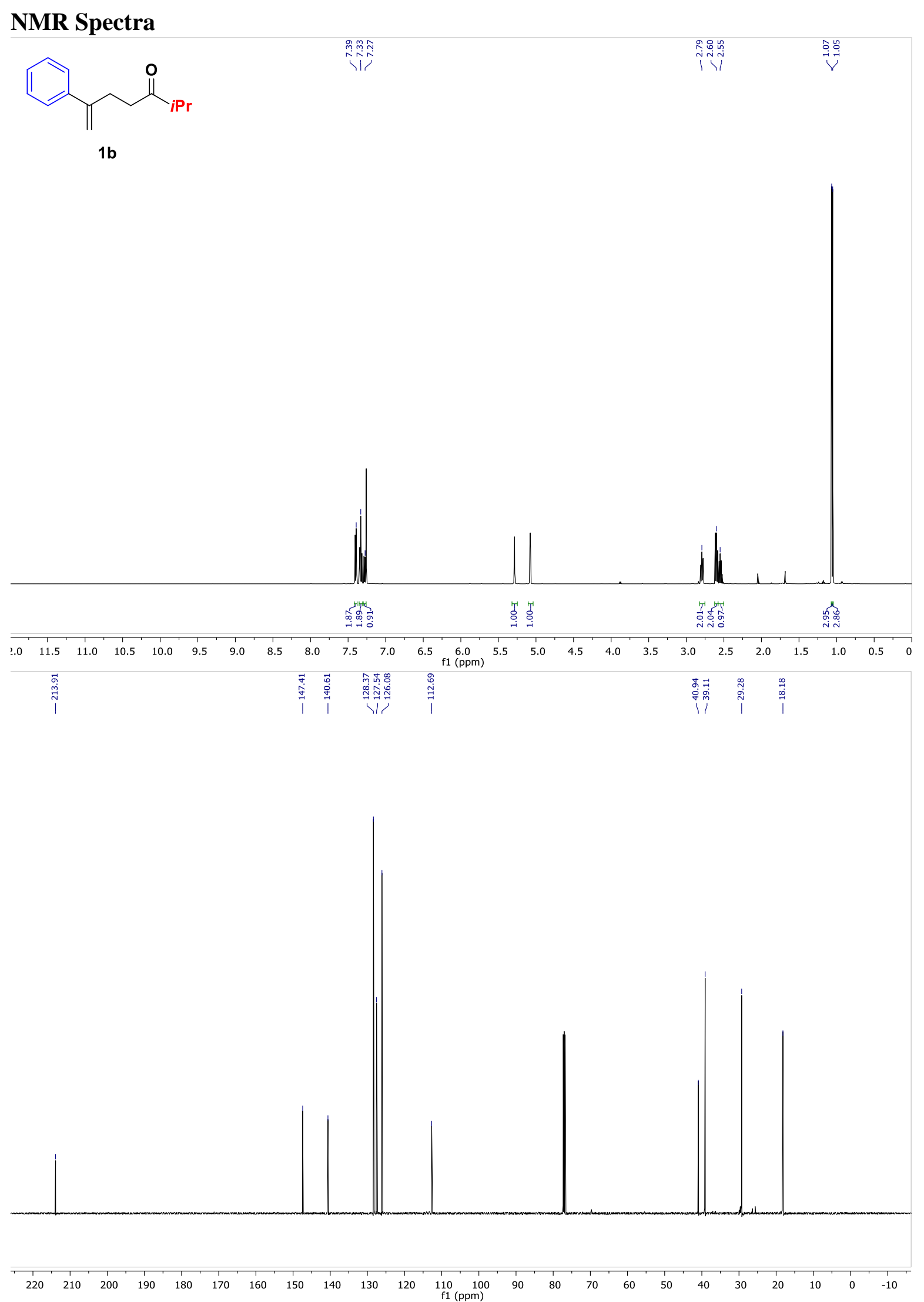

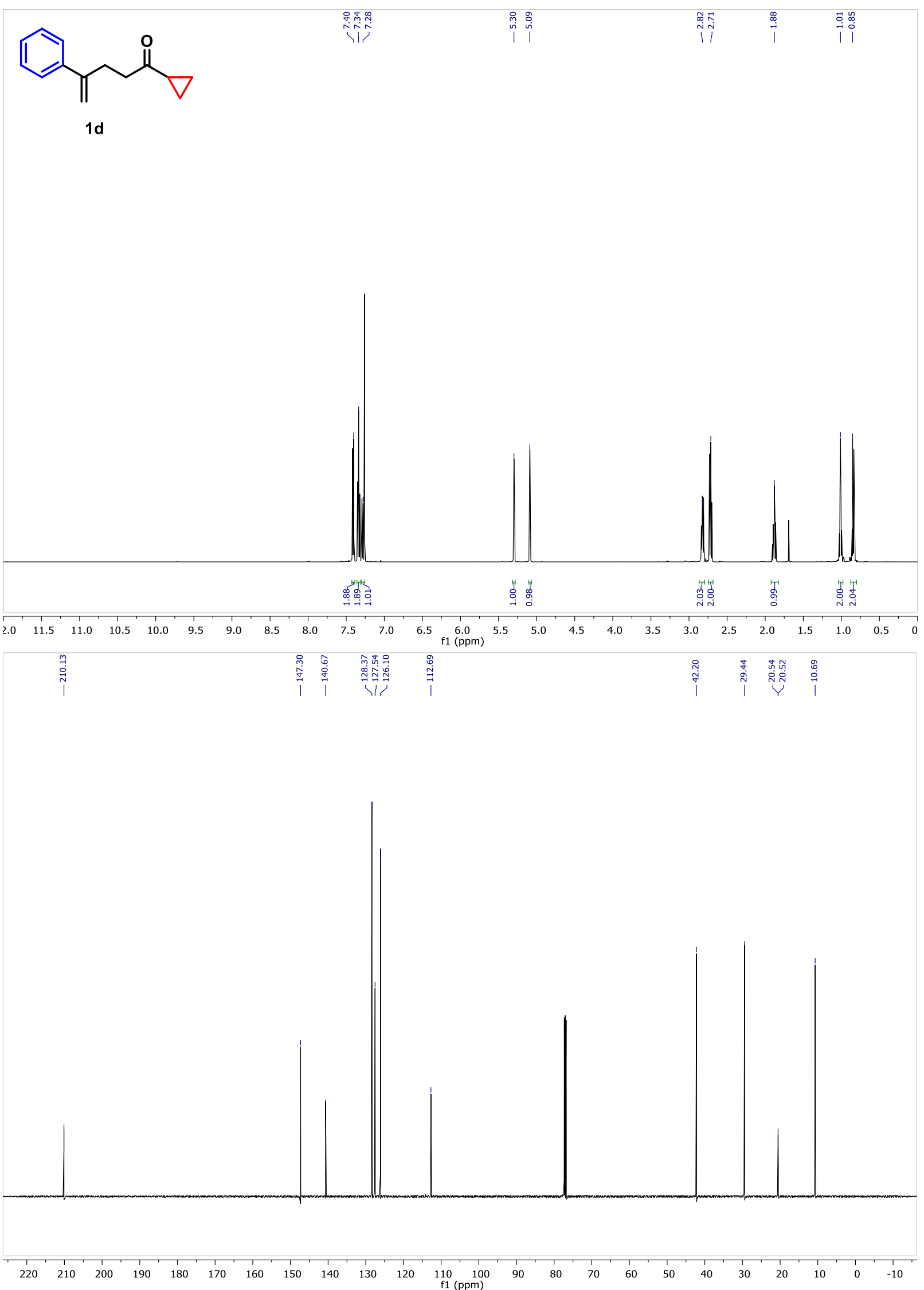

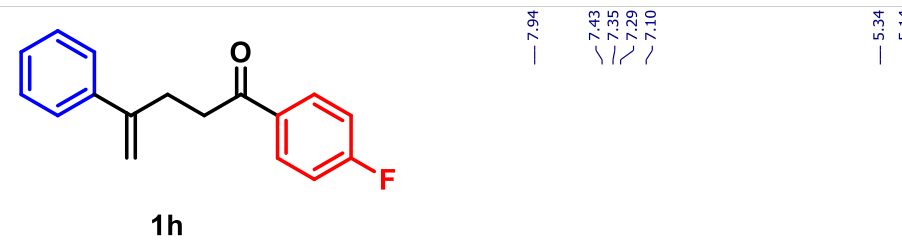

ติ
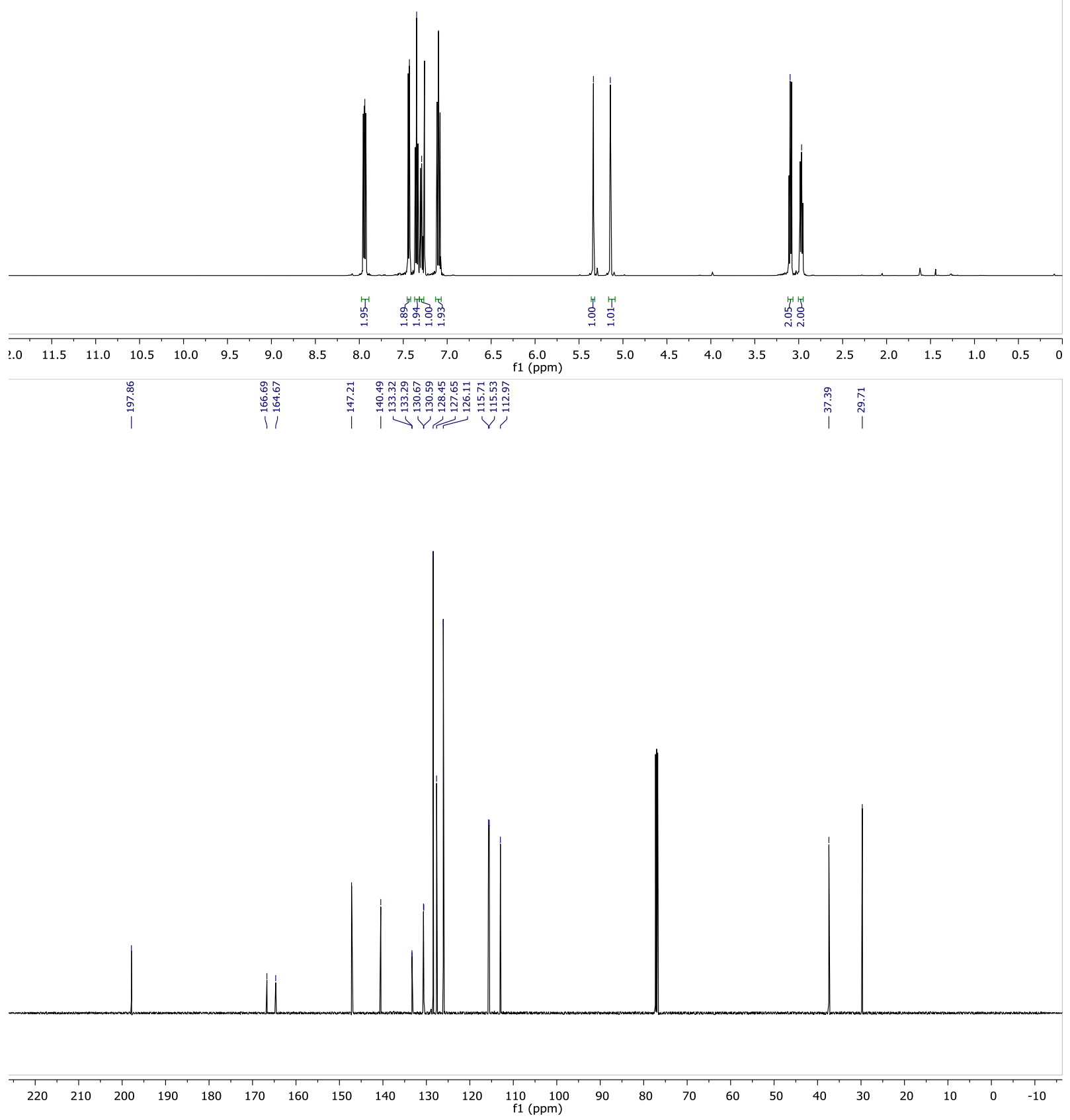

72 


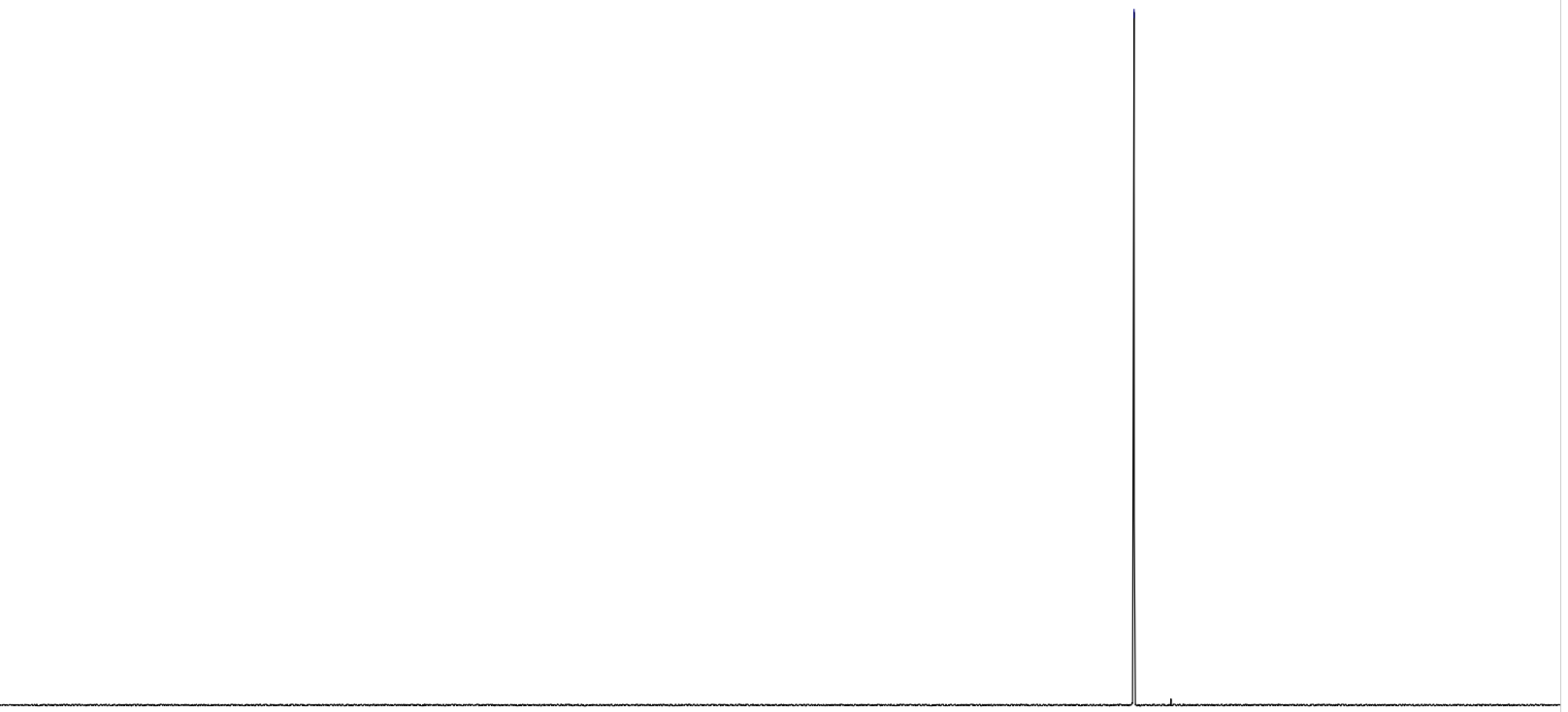

$\begin{array}{llllllllllllllllllllllll}170 & 150 & 130 & 110 & 90 & 70 & 50 & 30 & 10 & -10 & -30 & -50 & -70 & -90 & -110 & -130 & -150 & -170 & -190 & -211\end{array}$ 

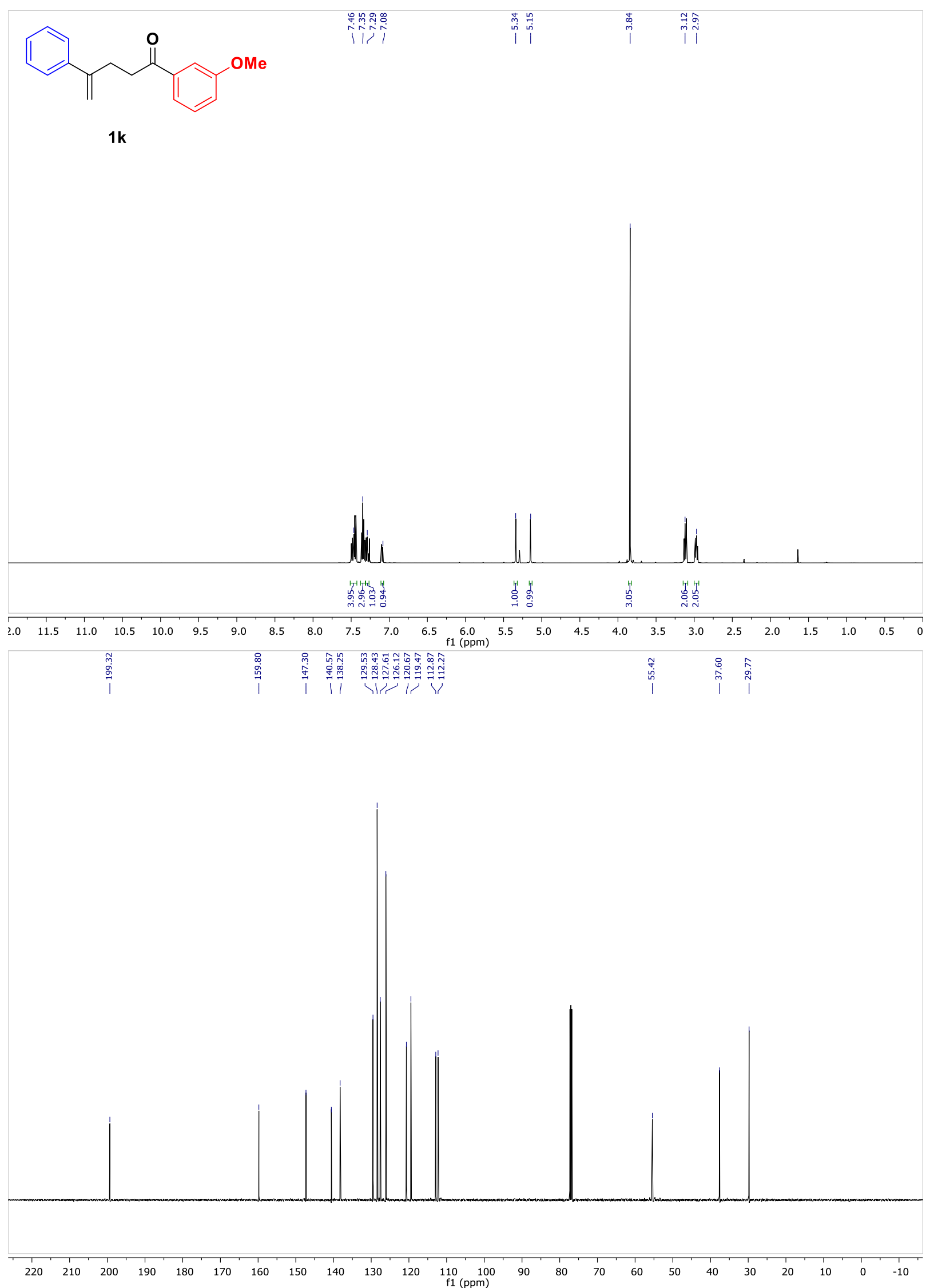

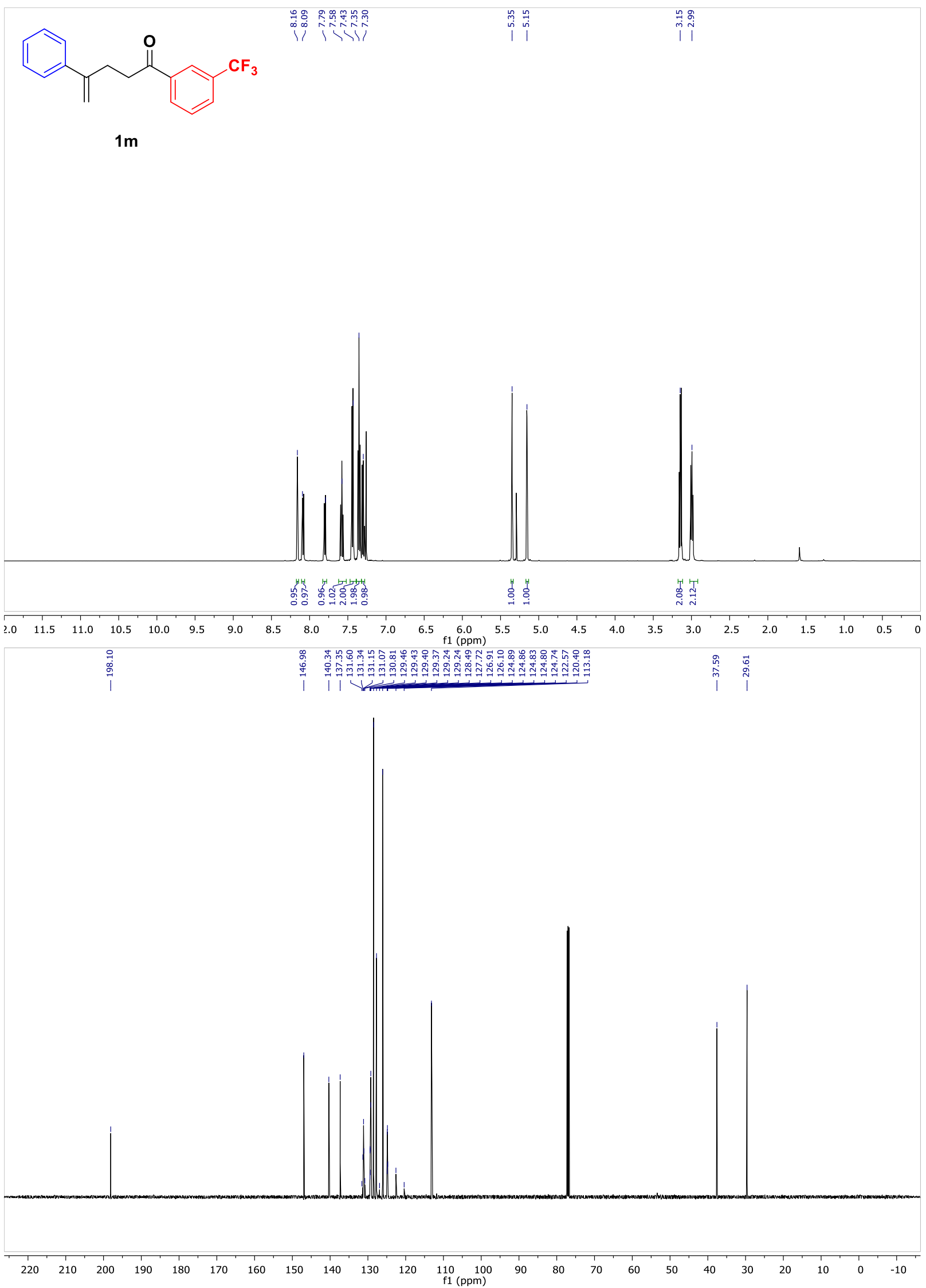


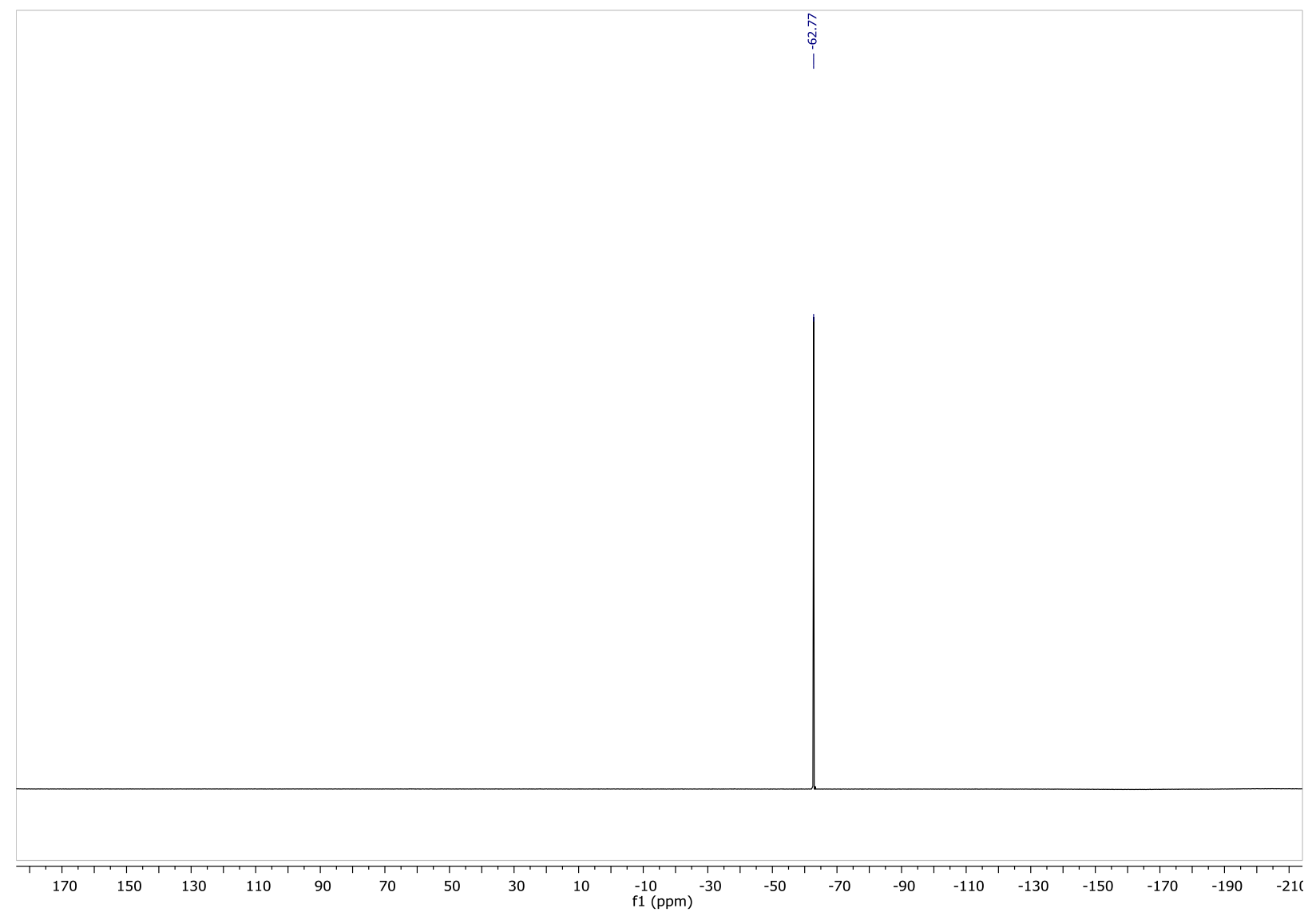



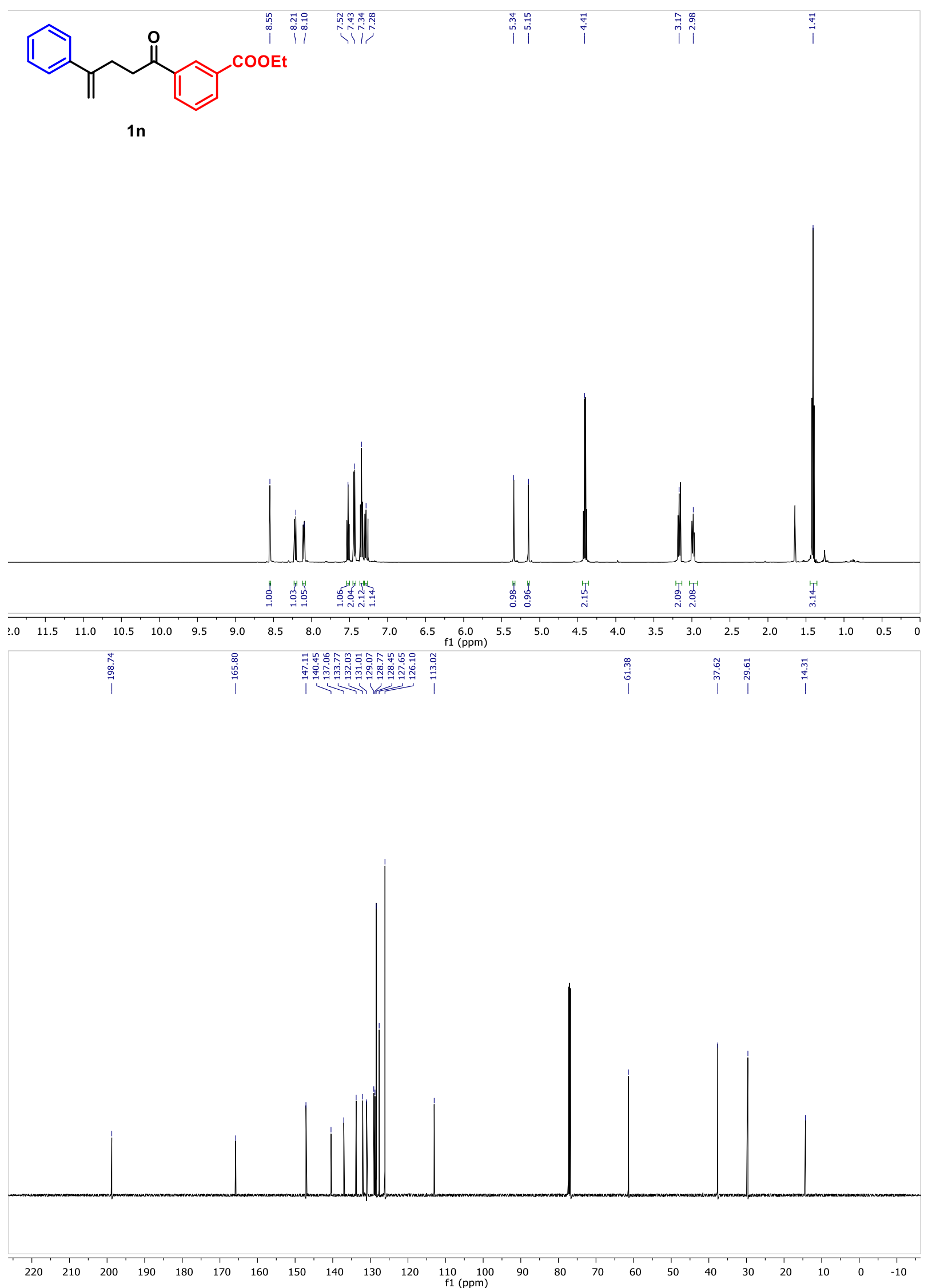

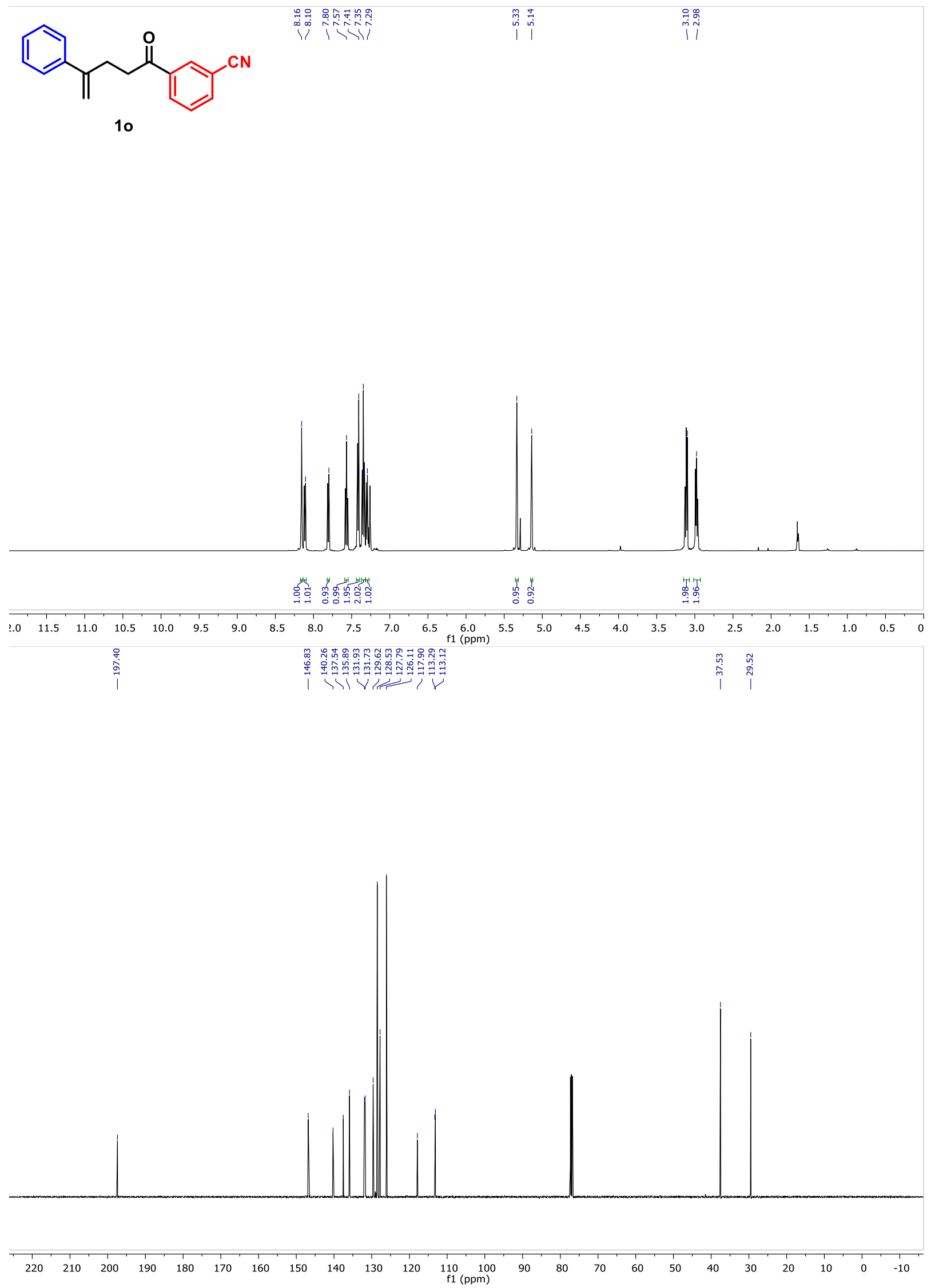


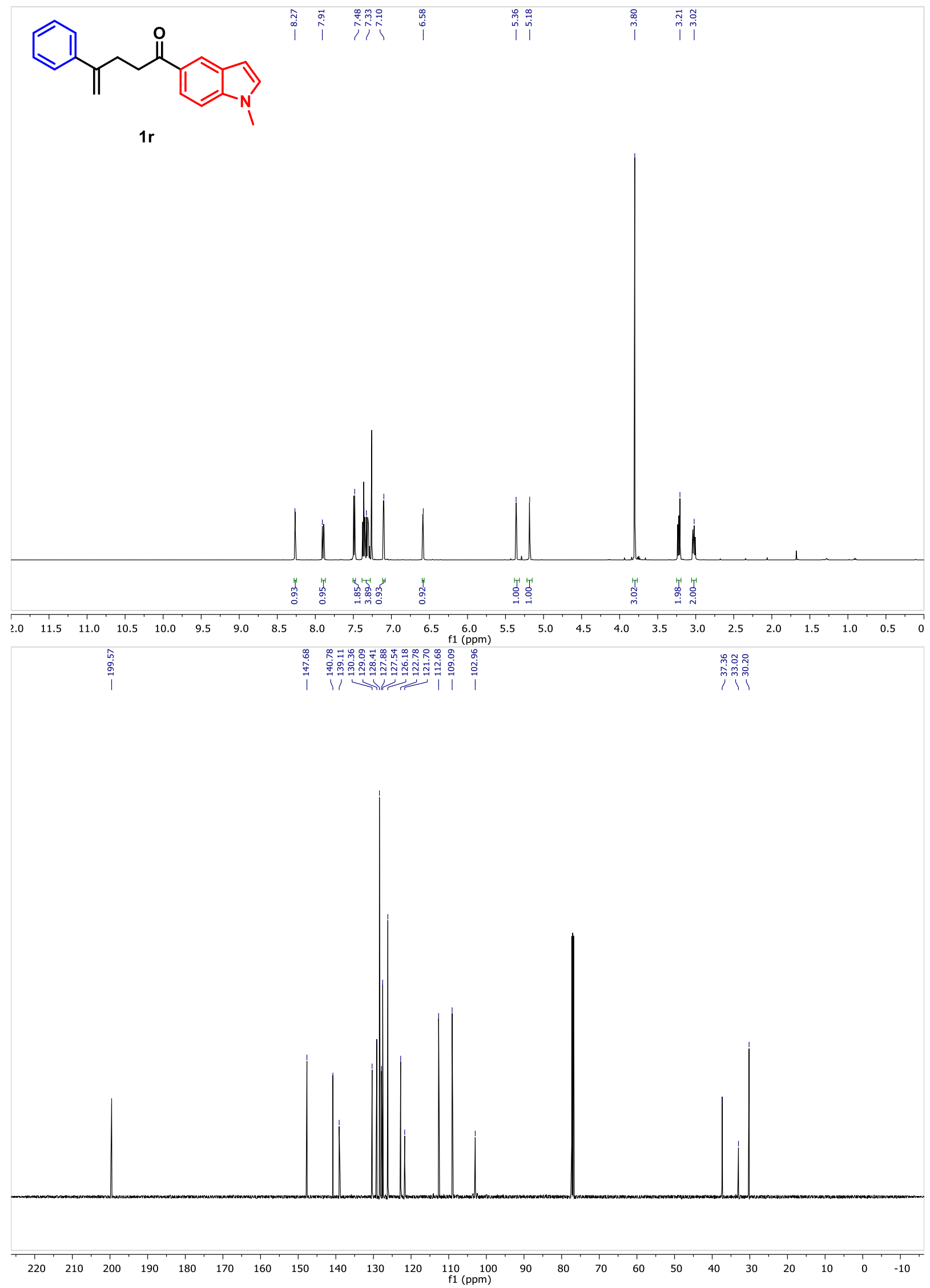



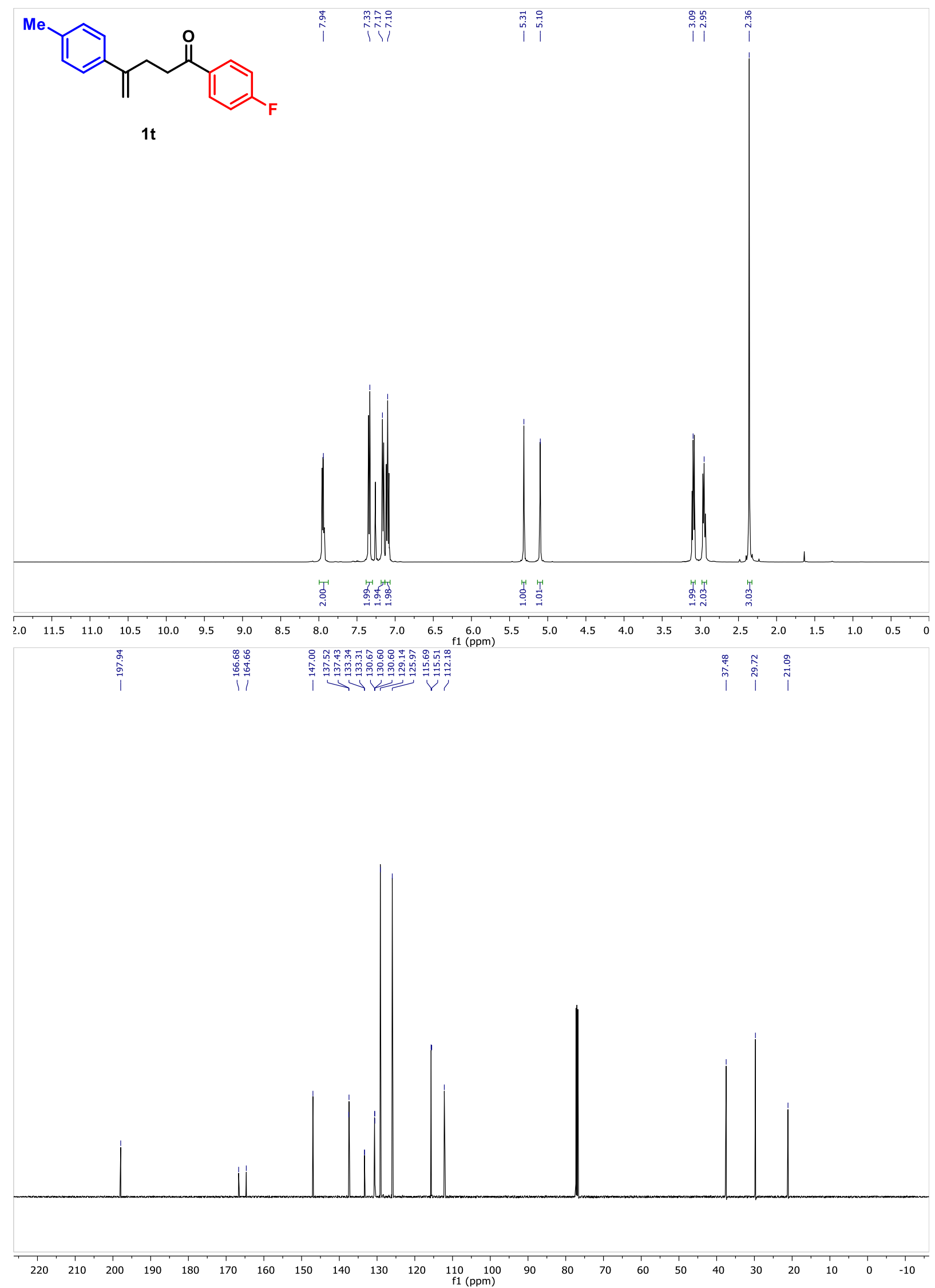


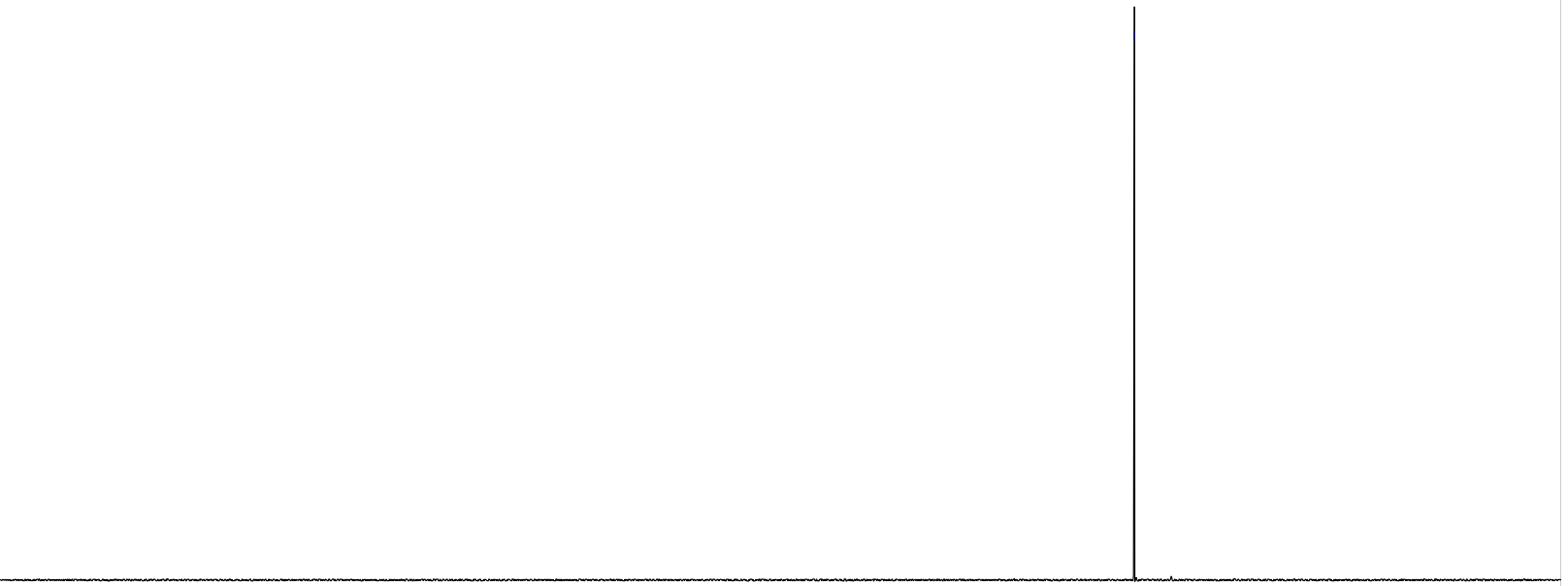

$\begin{array}{lllllllllllllllllllll}170 & 150 & 130 & 110 & 90 & 70 & 50 & 30 & 10 & -10 & -30 & -50 & -70 & -90 & -110 & -130 & -150 & -170 & -190 & -211\end{array}$




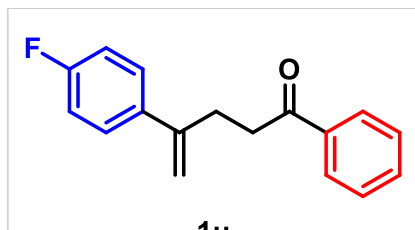

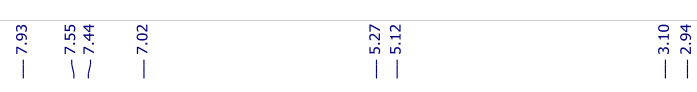

$1 \mathrm{u}$
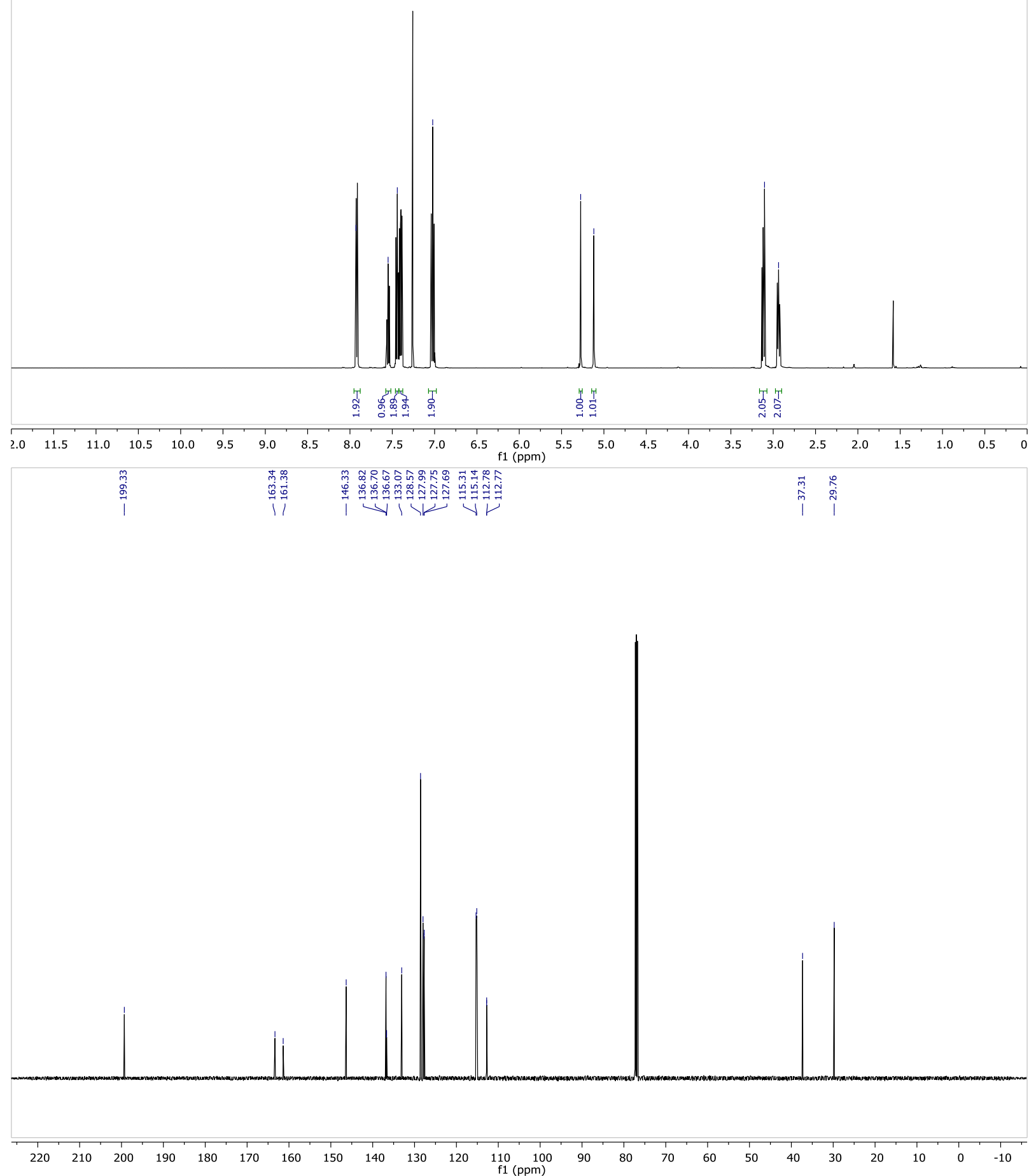


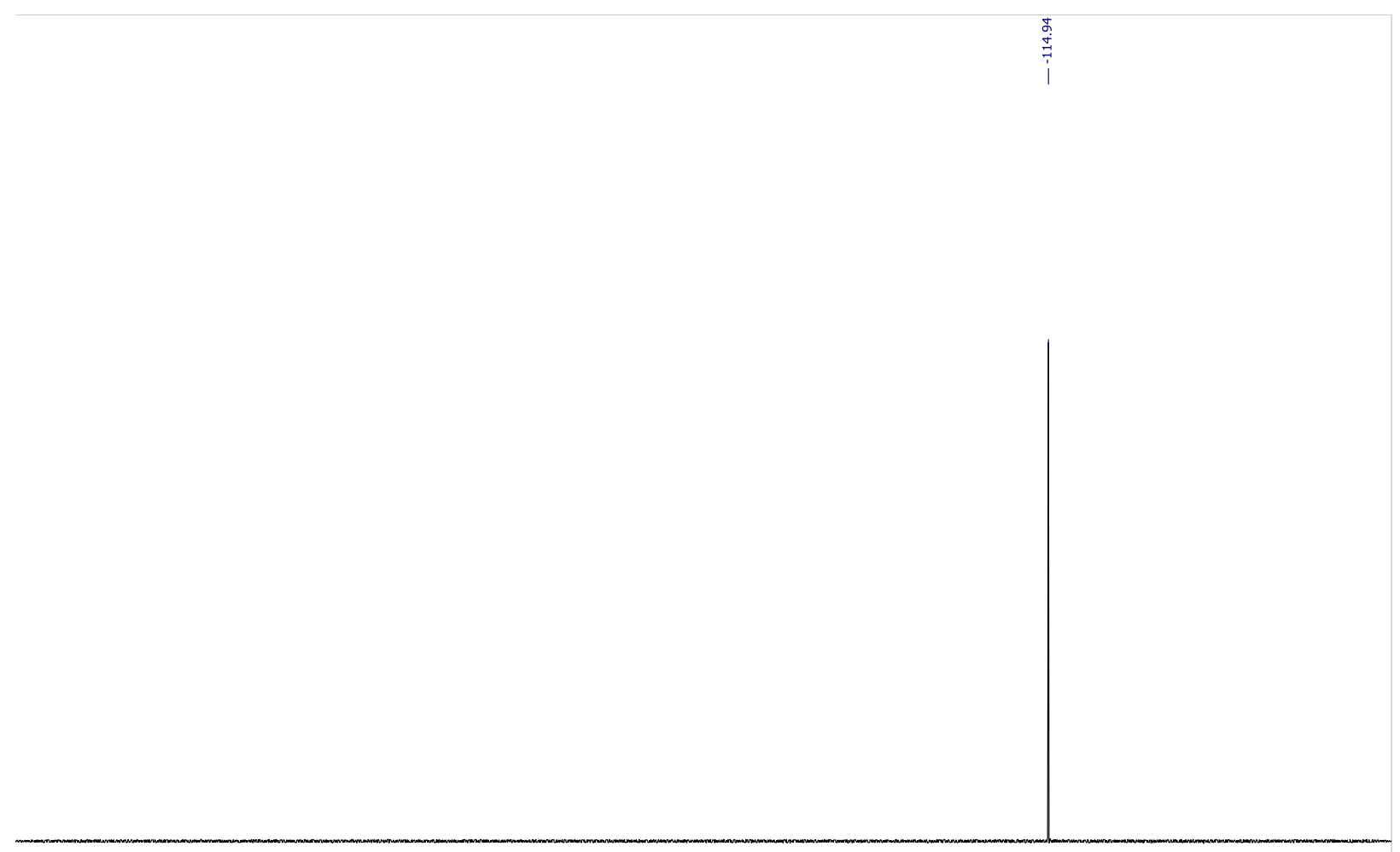

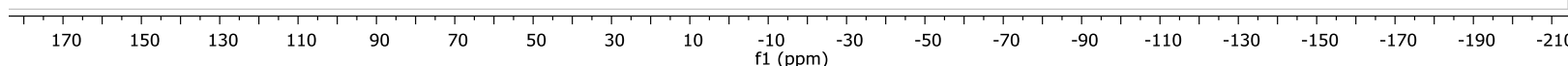



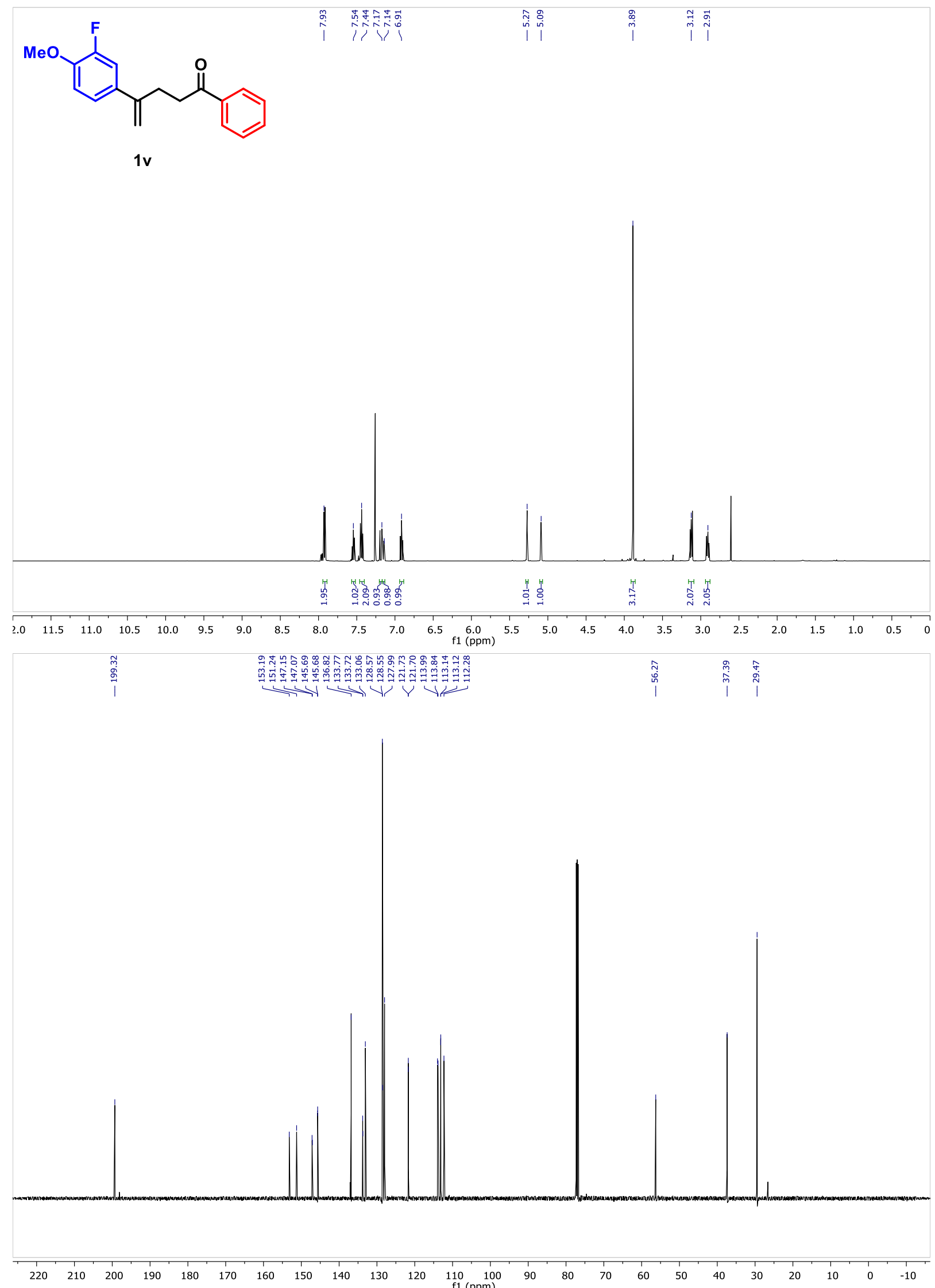


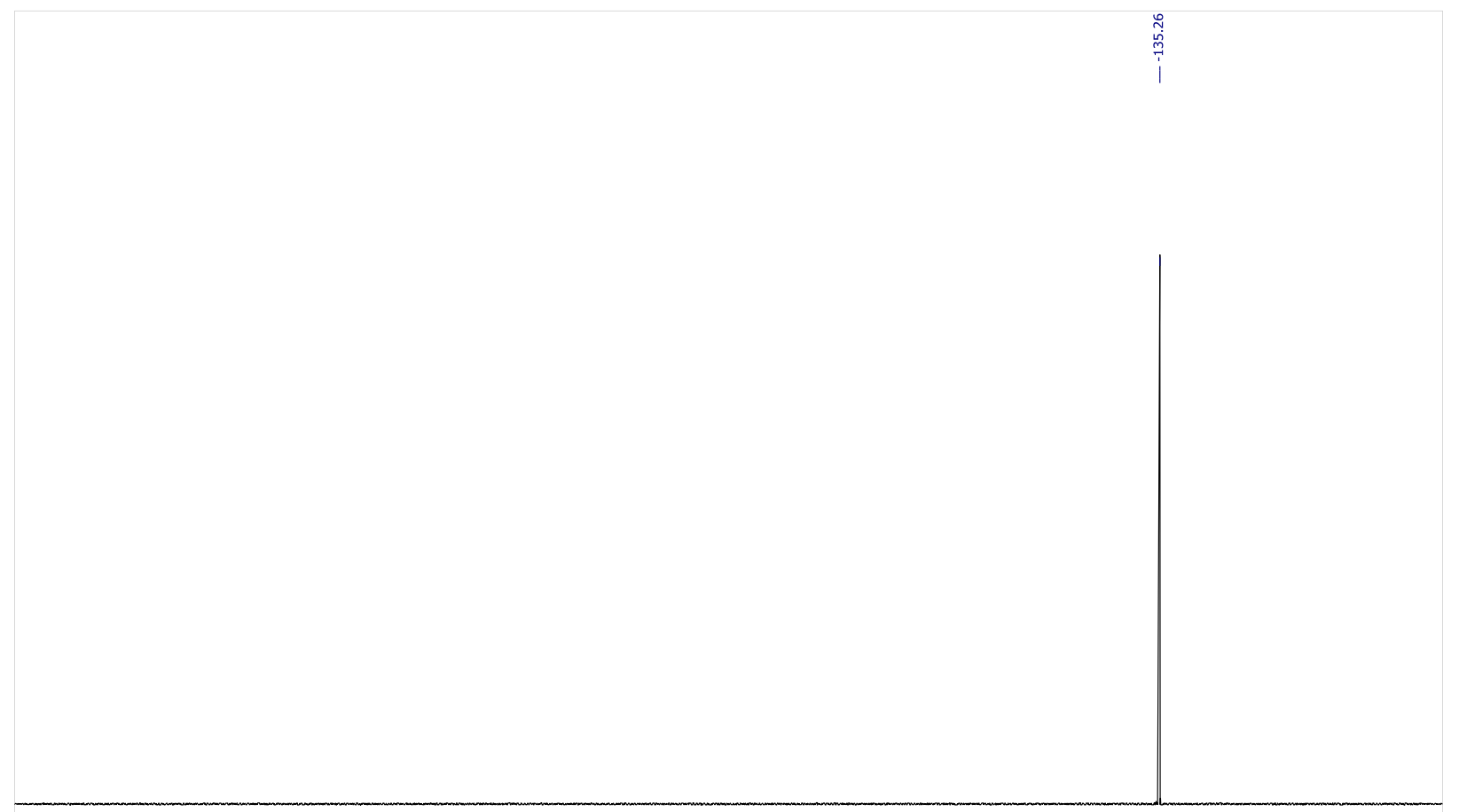

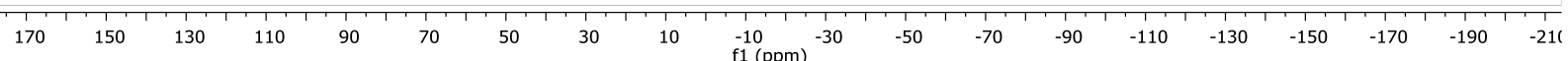




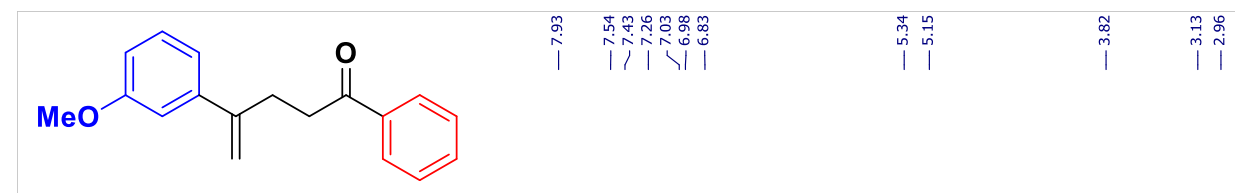

$1 x$
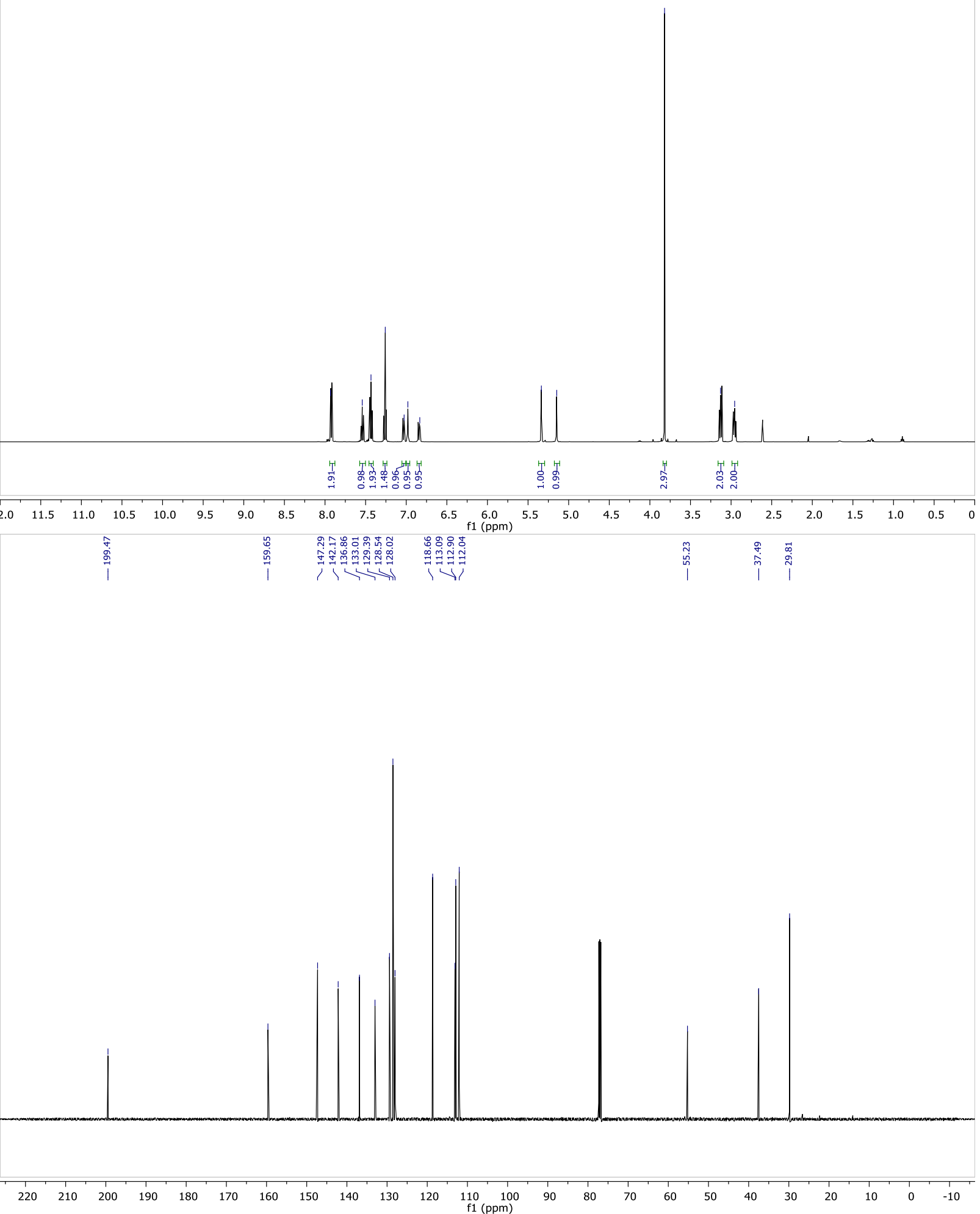
<smiles>C=C(CCC(=O)c1ccccc1)c1cccc(F)c1</smiles>

$1 y$
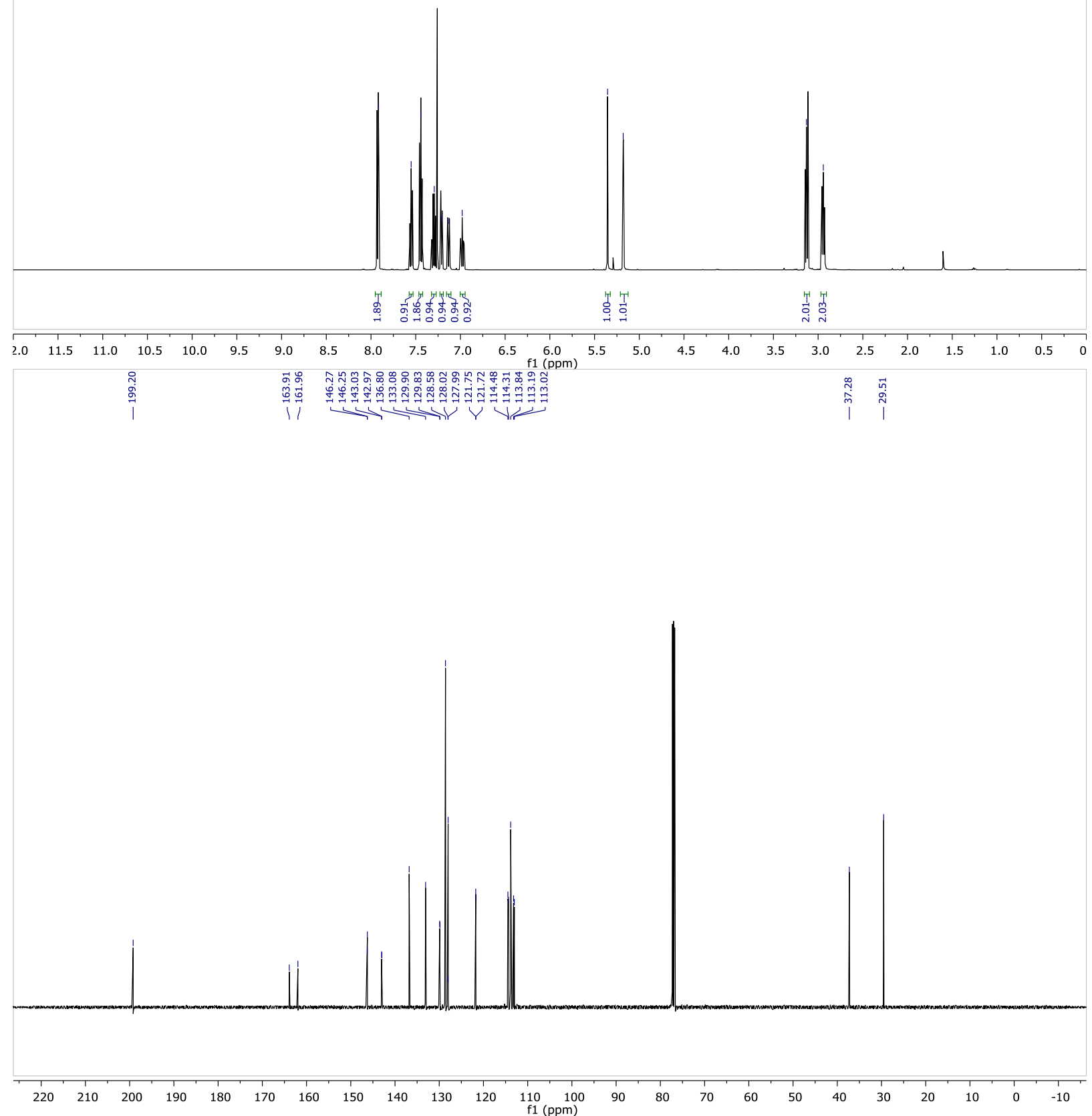


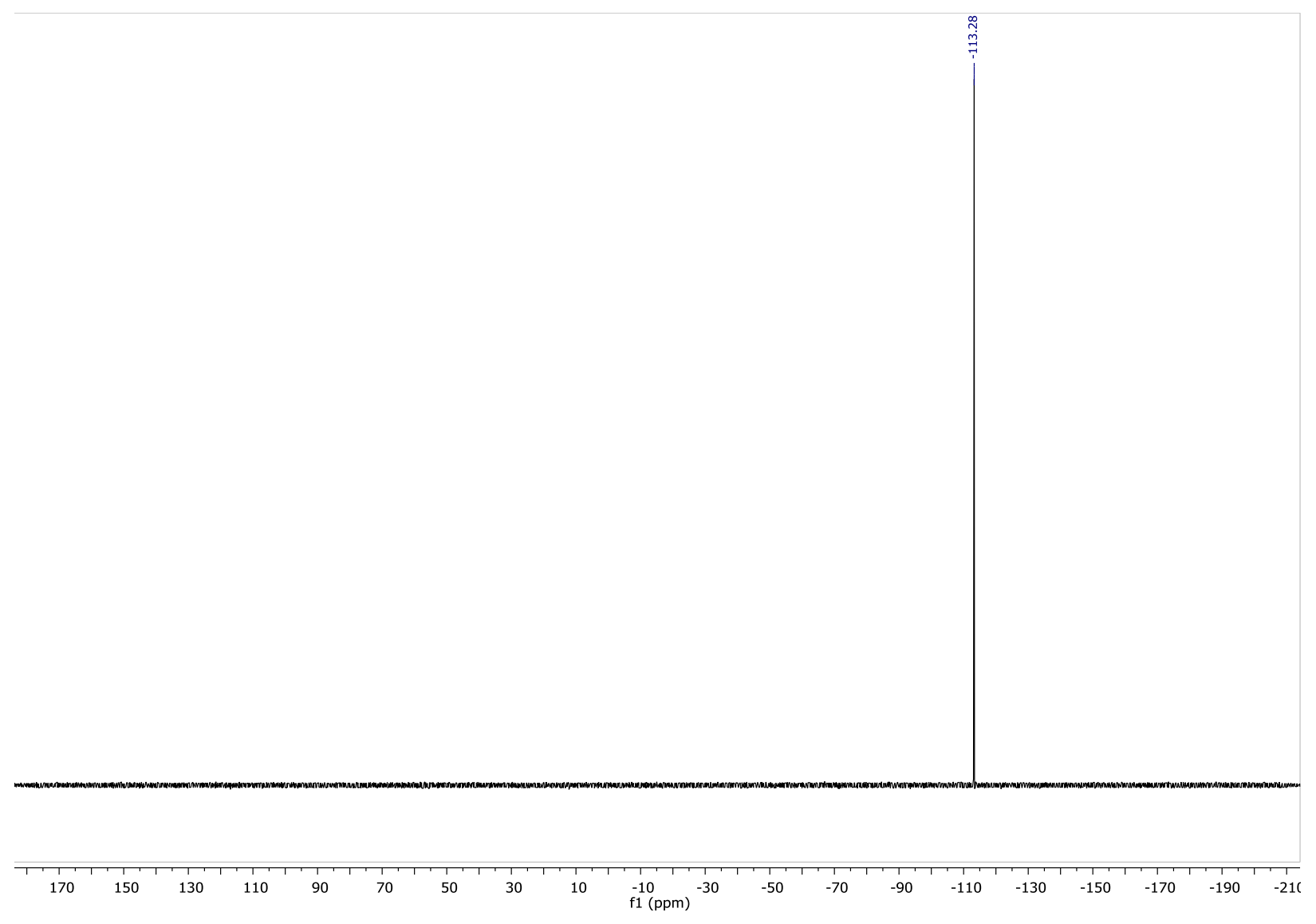


<smiles>C=C(CCC(=O)c1ccccc1)c1cccc(C(F)(F)F)c1</smiles>

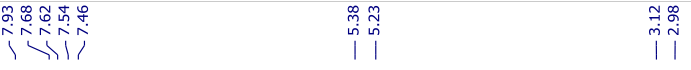

$1 z$
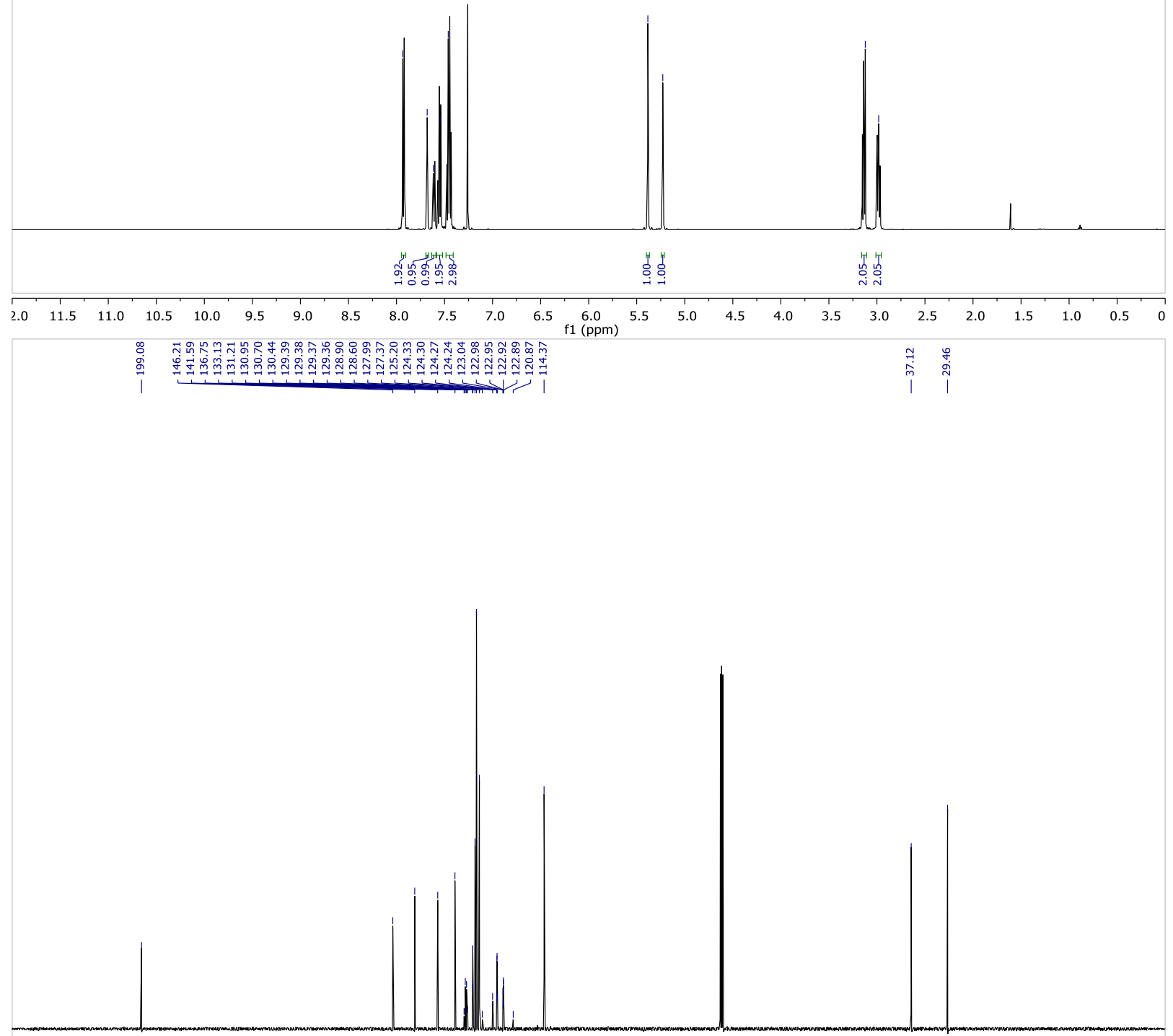

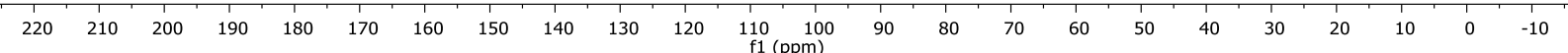




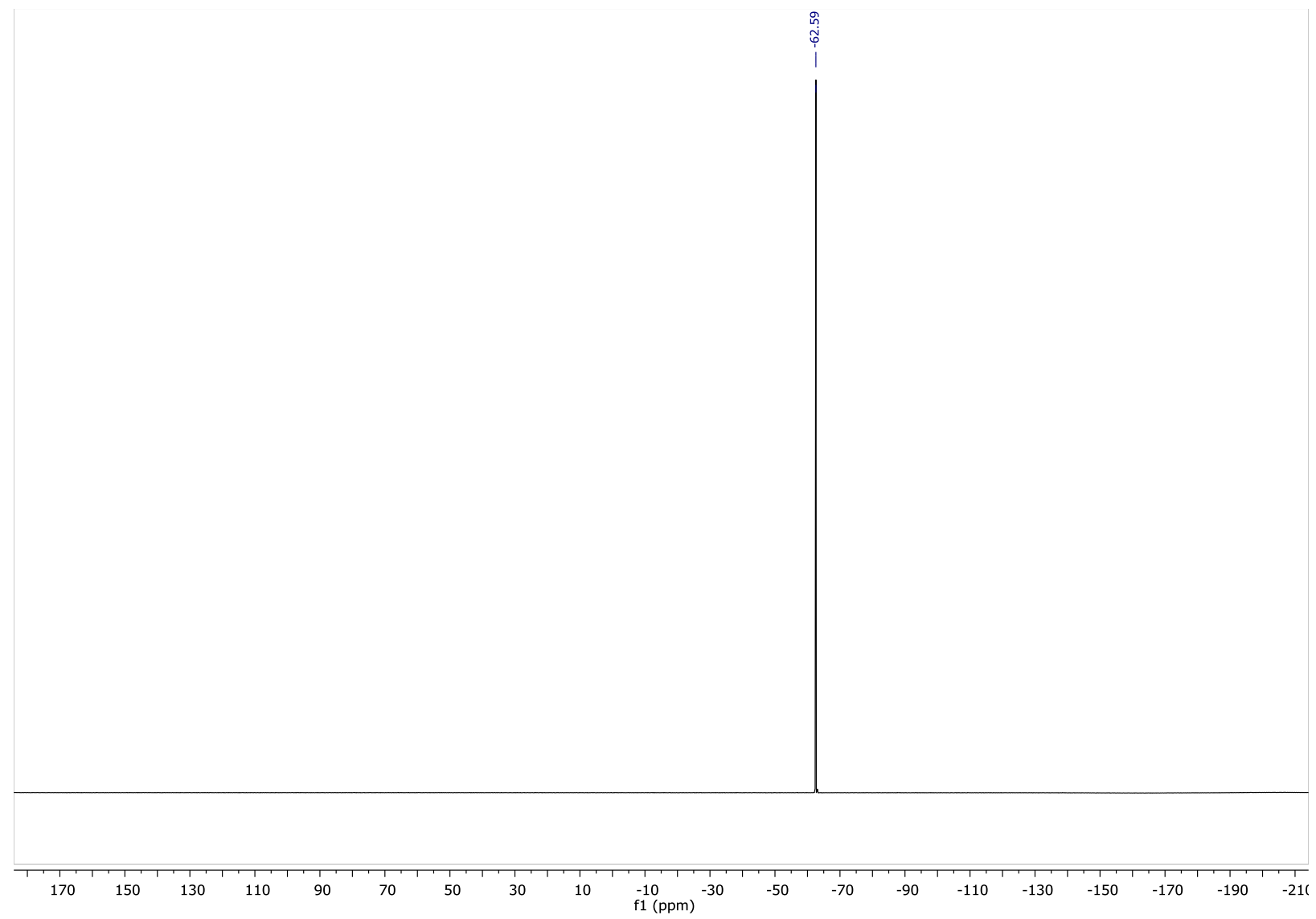



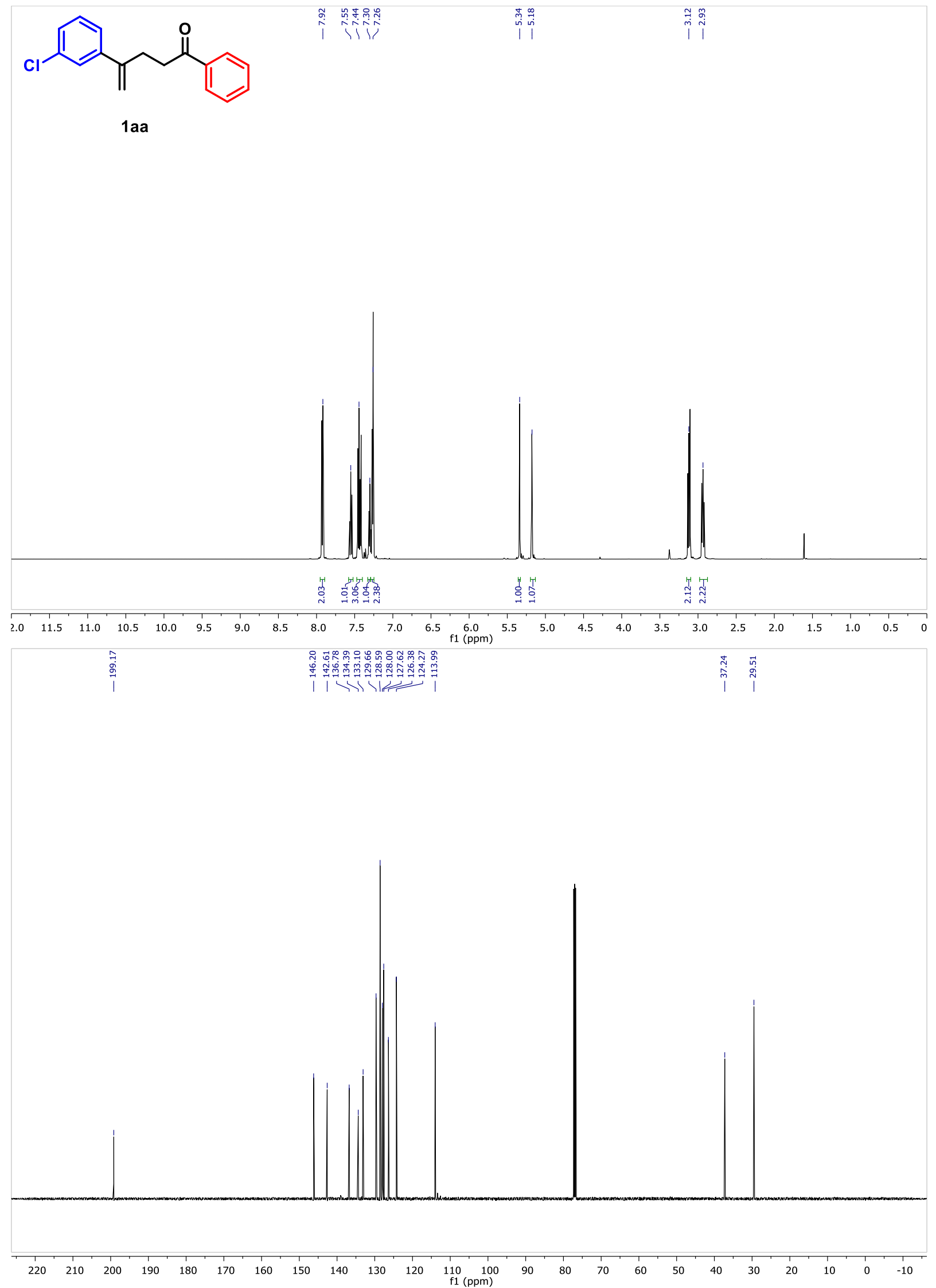

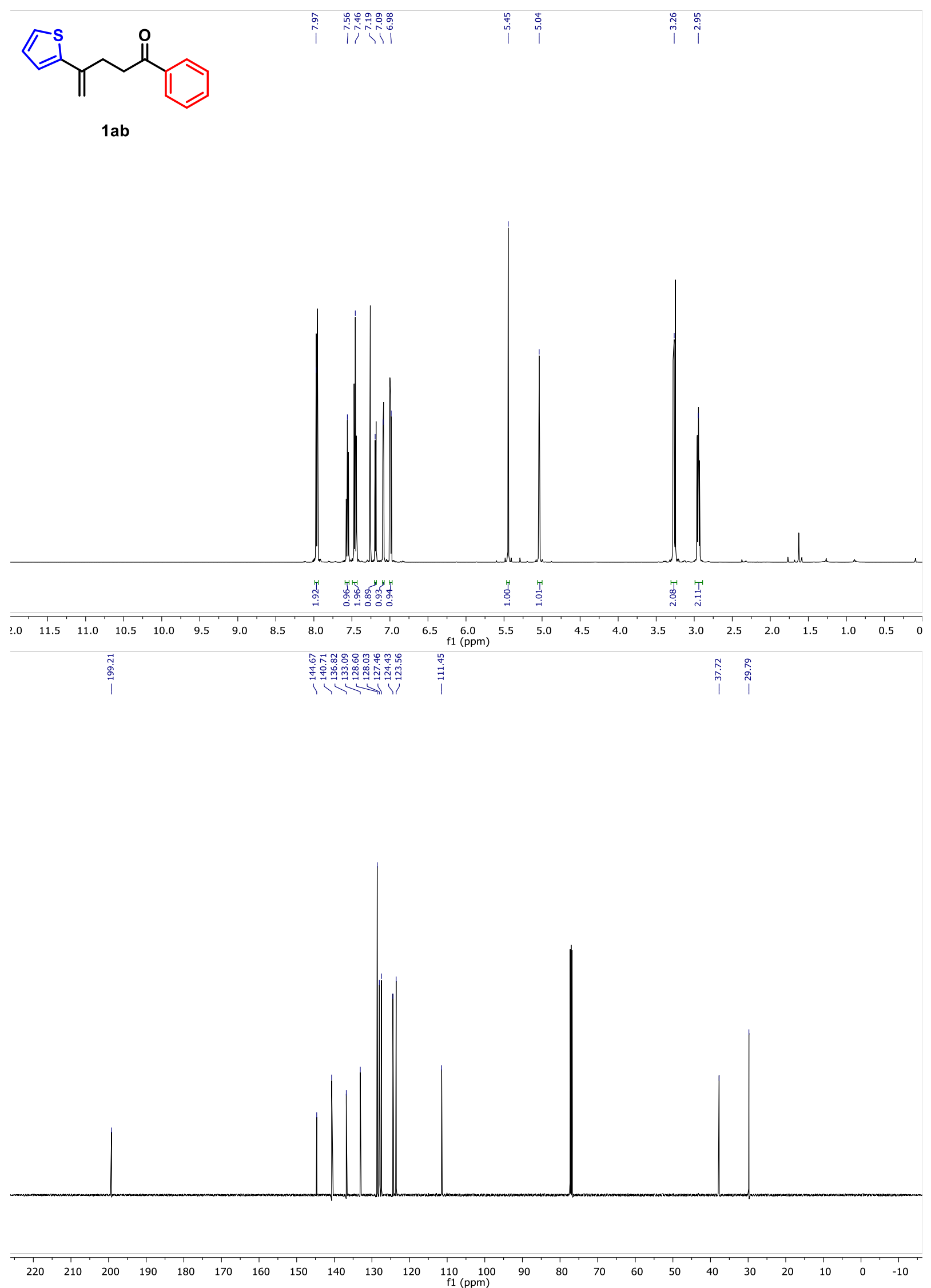

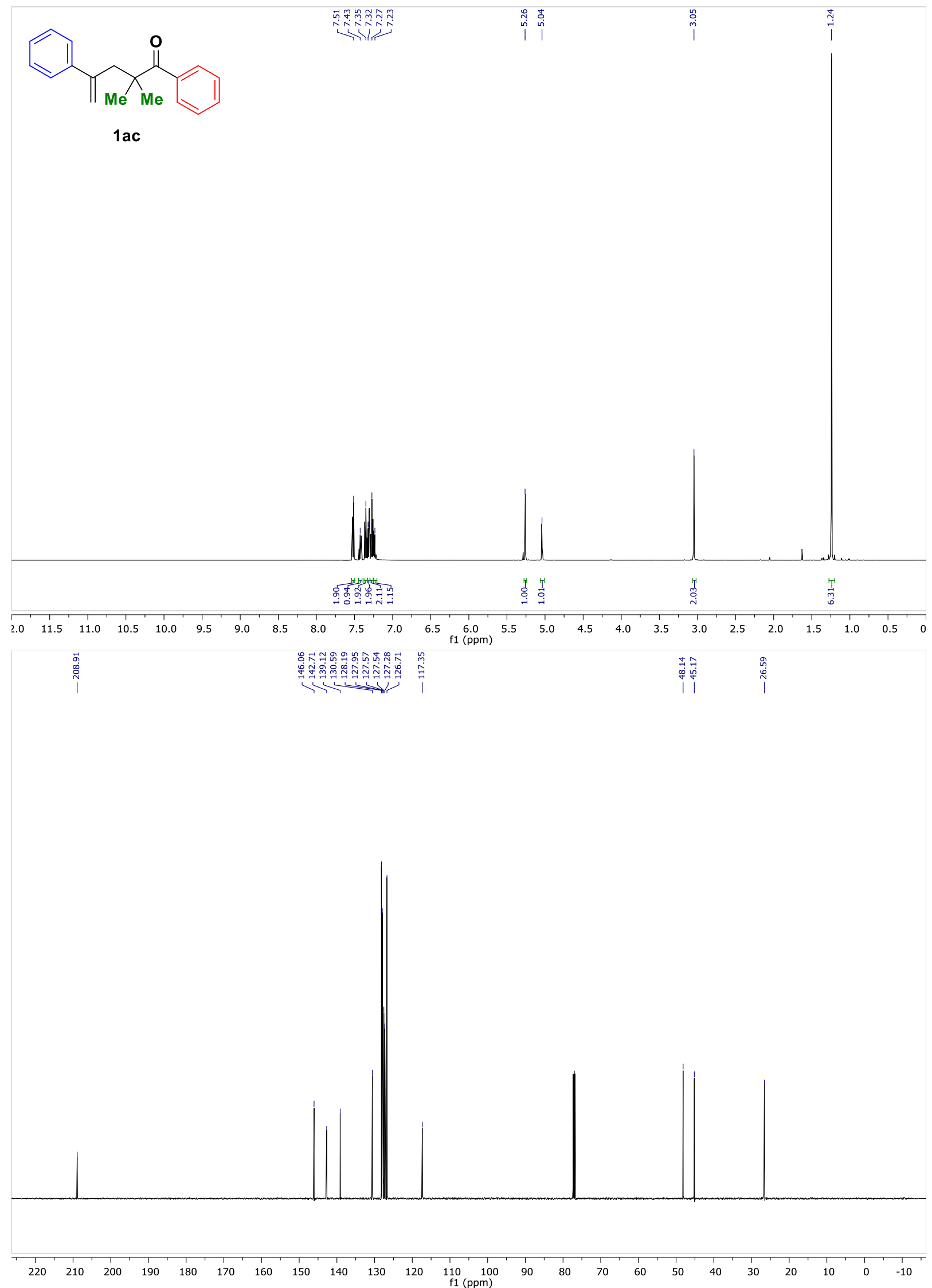

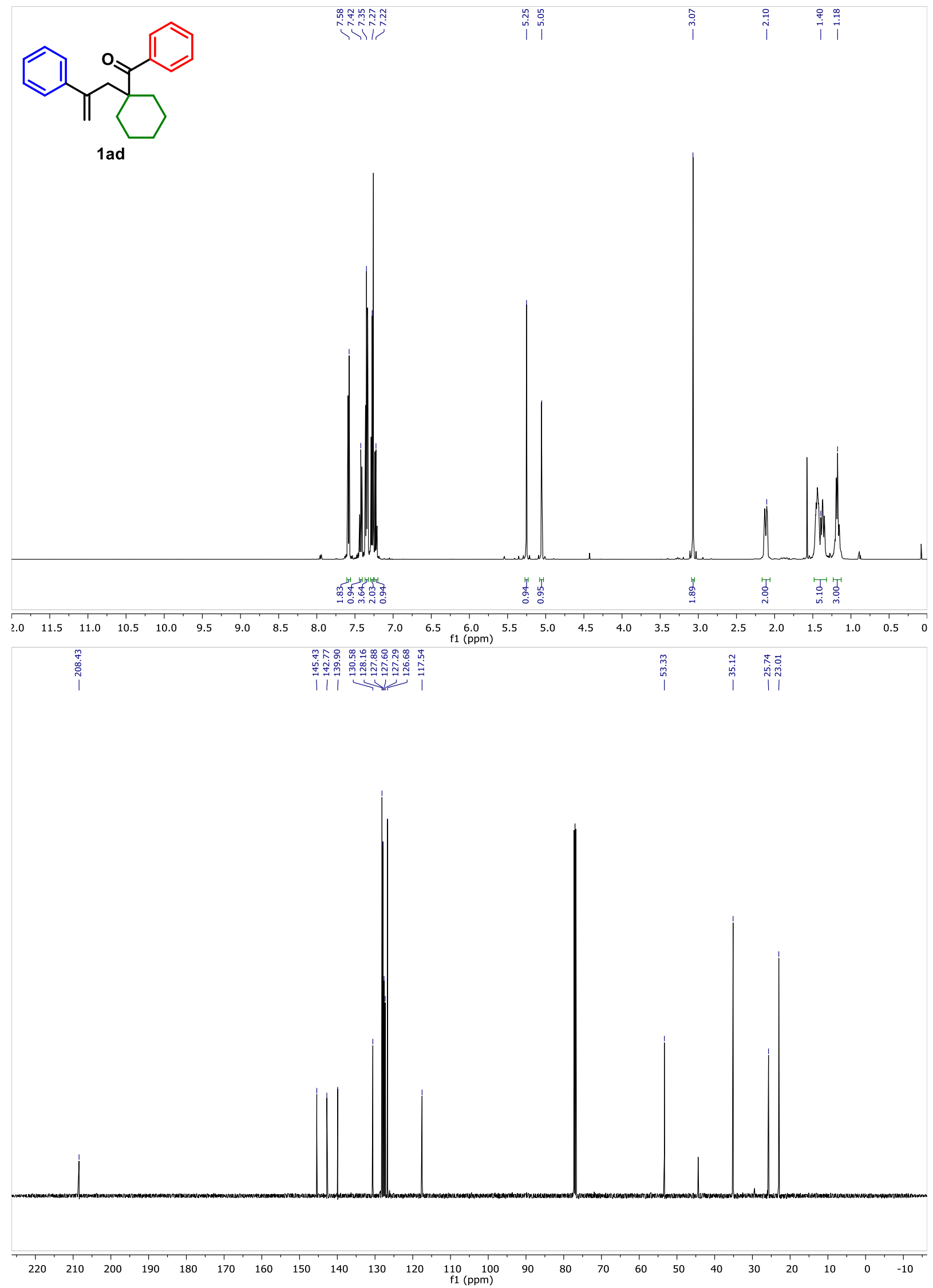

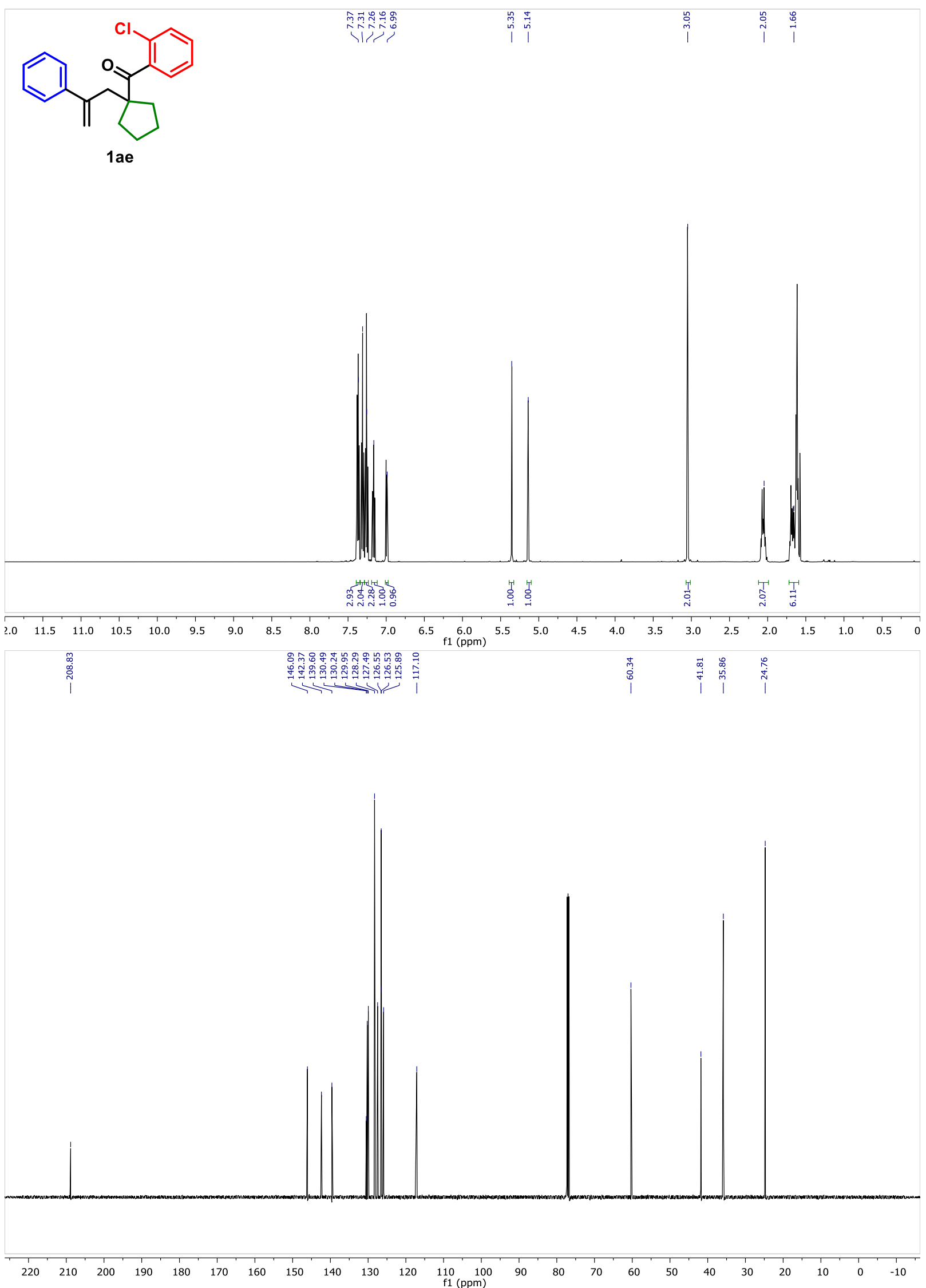

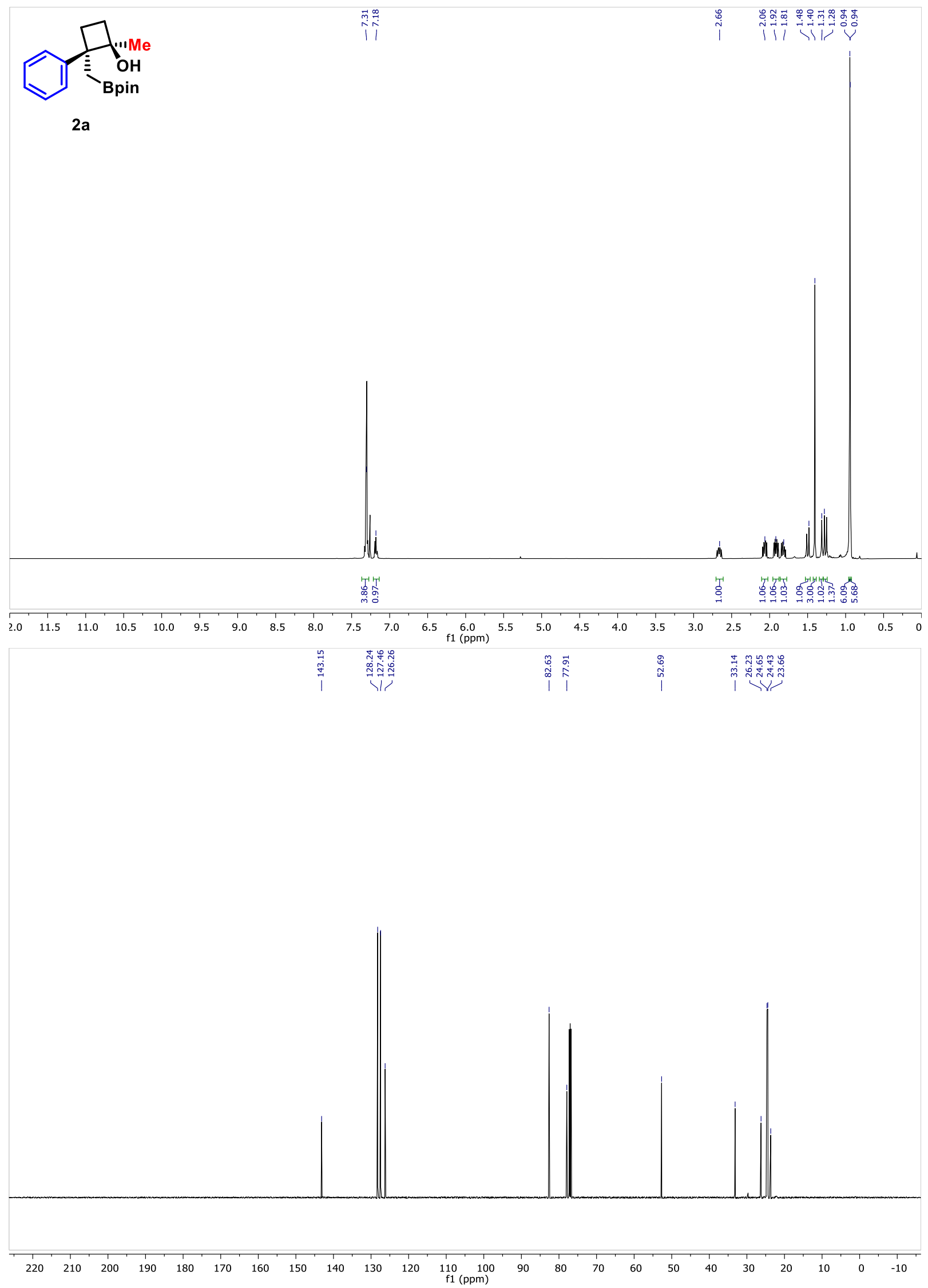


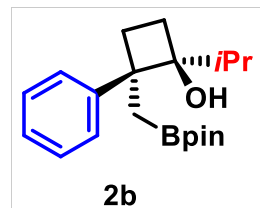

$2 b$
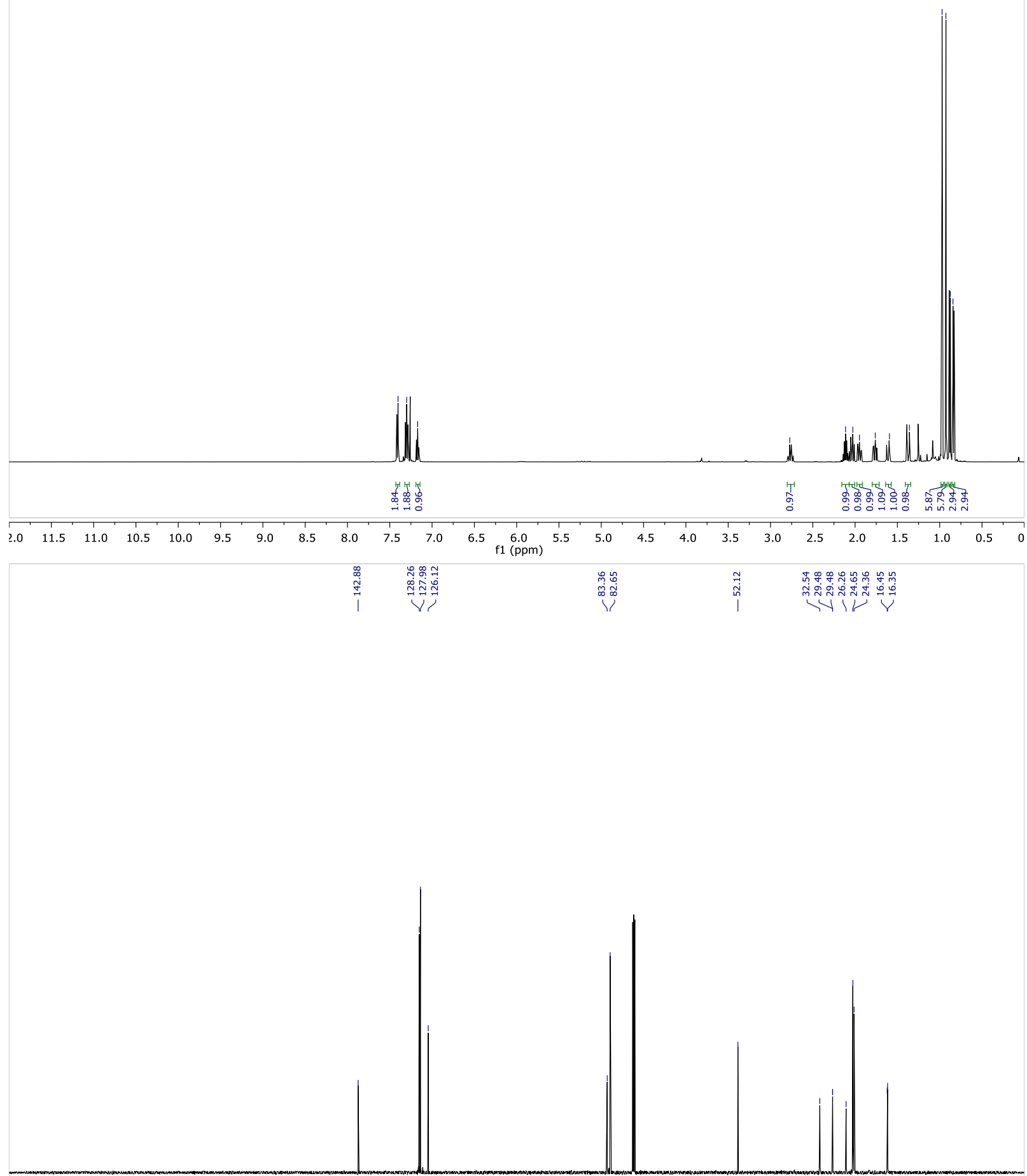

$\begin{array}{llllllllllll}220 & 210 & 200 & 190 & 180 & 170 & 160 & 150 & 140 & 130 & 120 & \begin{array}{c}110 \\ \mathrm{f} 1(\mathrm{ppm})\end{array}\end{array}$ 


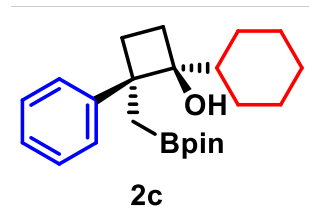

2c
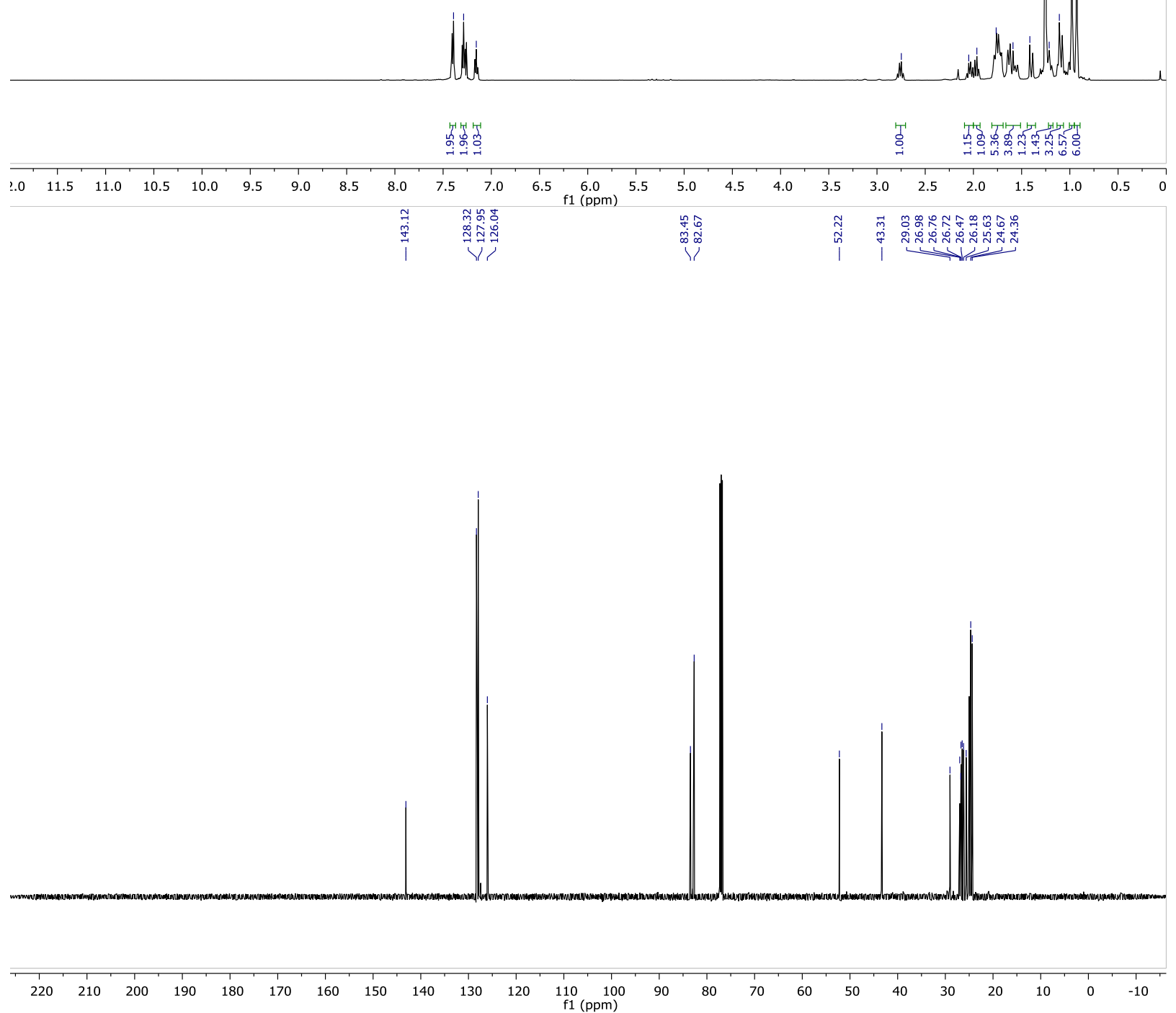


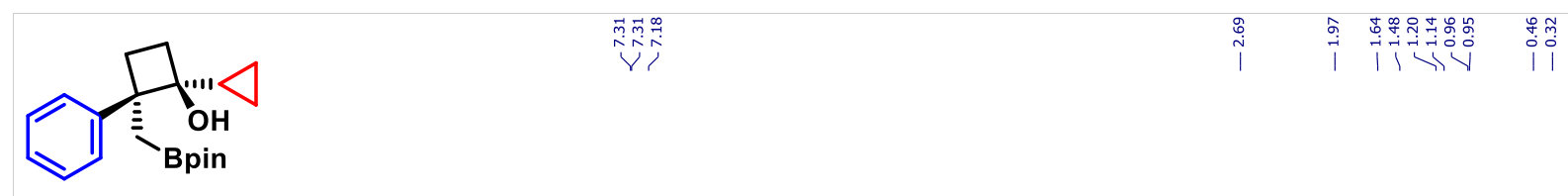

2d
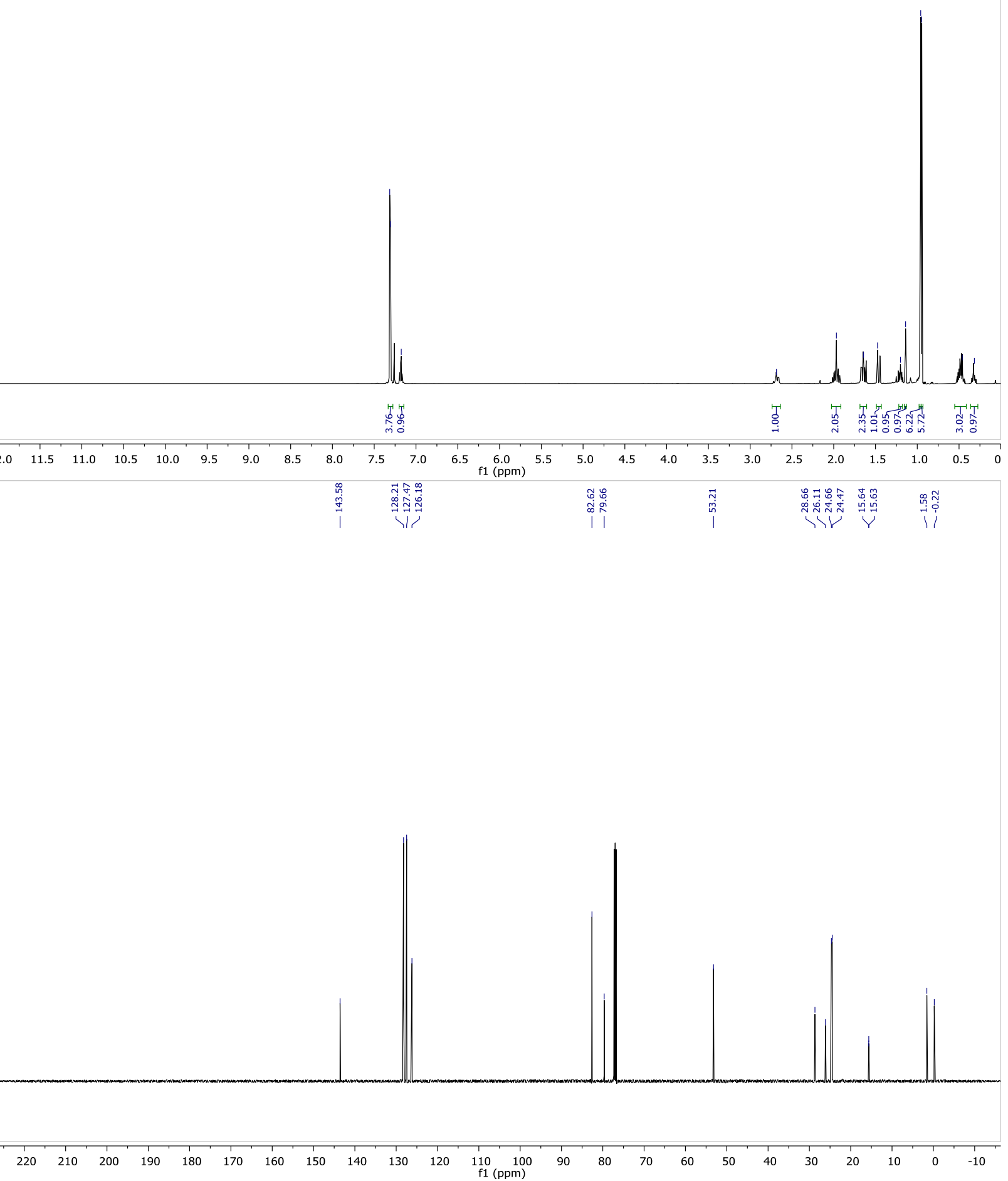

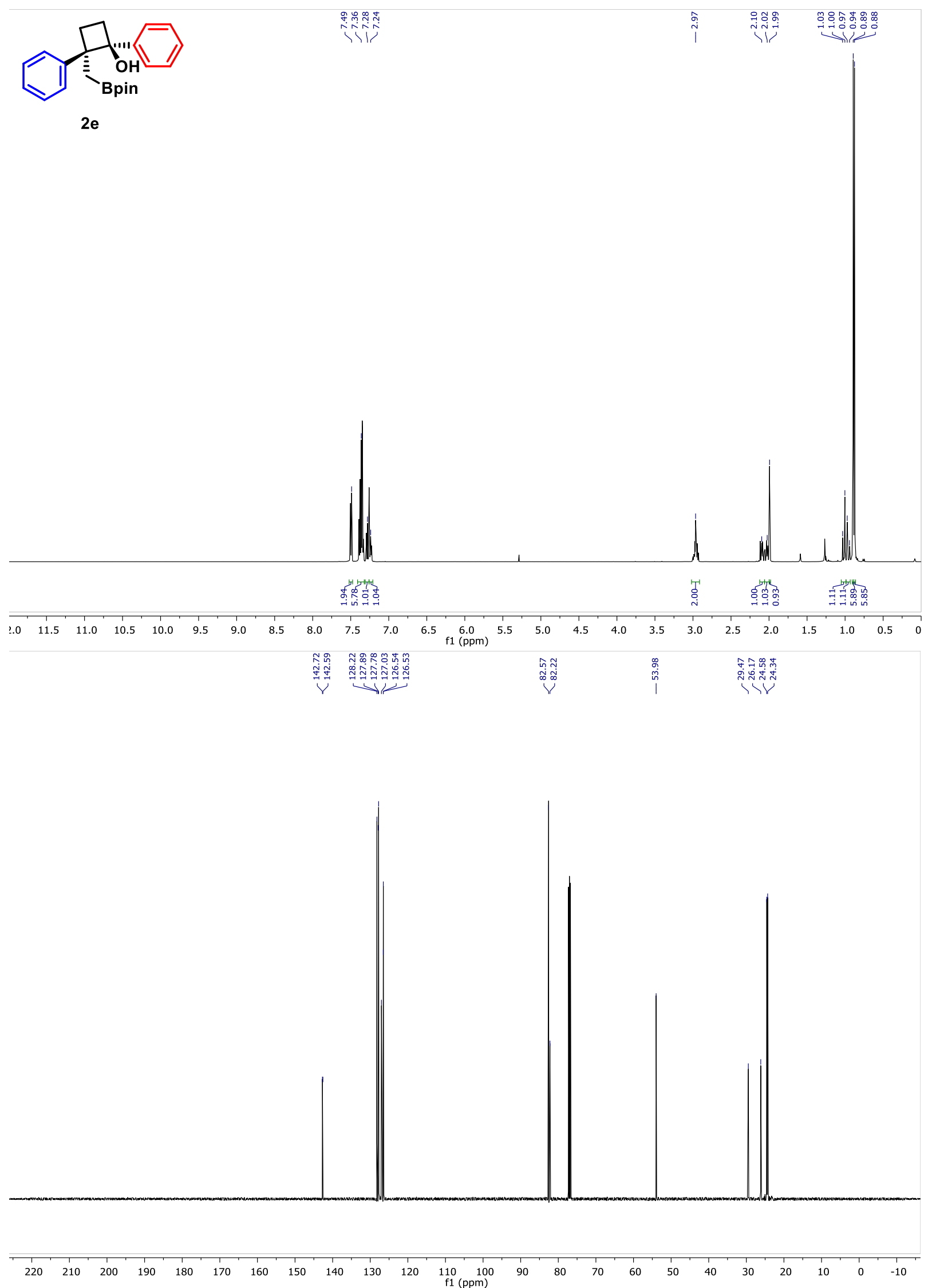

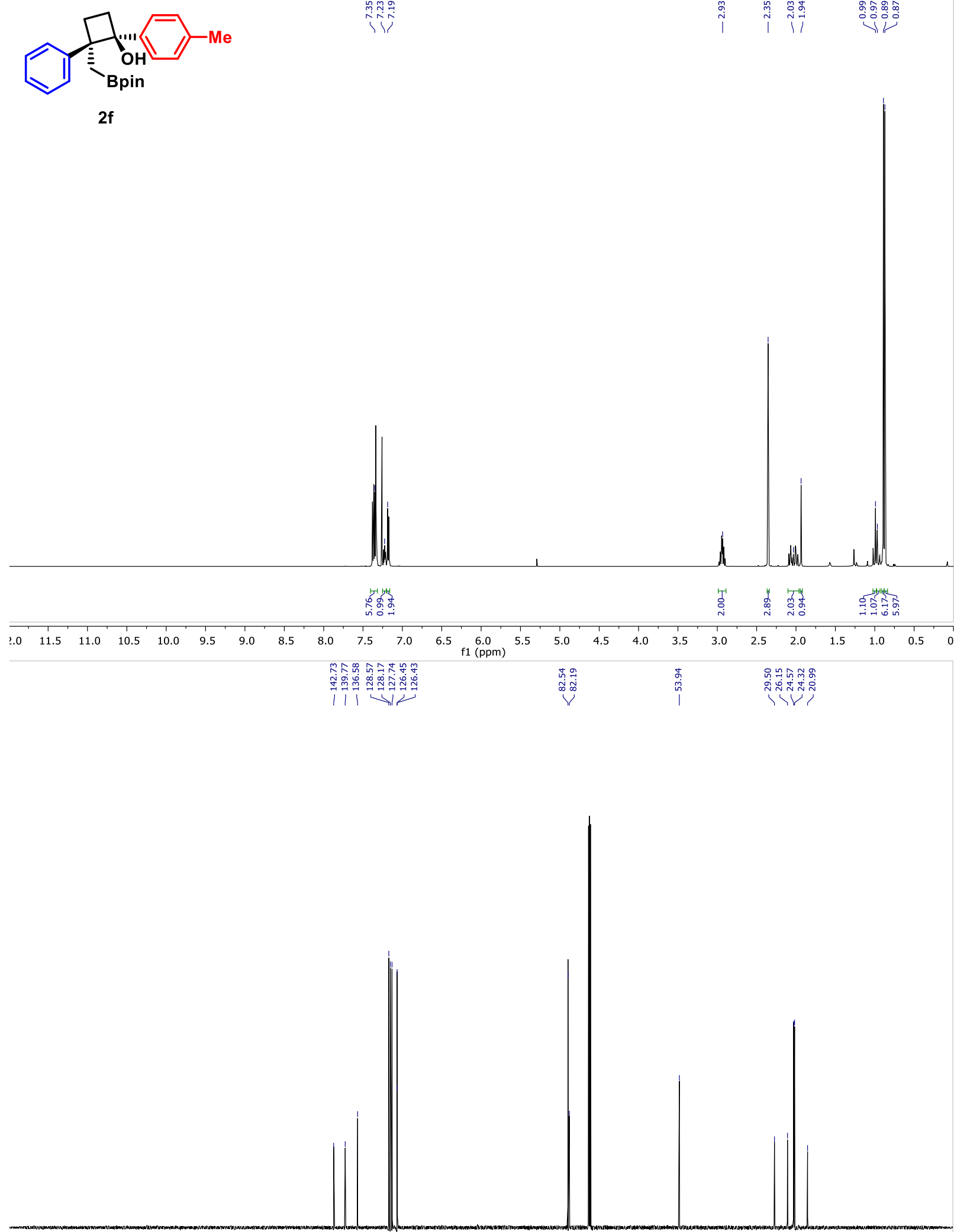

$\begin{array}{llllllllllllllllllllllllllll}220 & 210 & 200 & 190 & 180 & 170 & 160 & 150 & 140 & 130 & 120 & 110 & 100 & 90 & 80 & 70 & 60 & 50 & 40 & 30 & 20 & 10 & 0 & -10\end{array}$ 

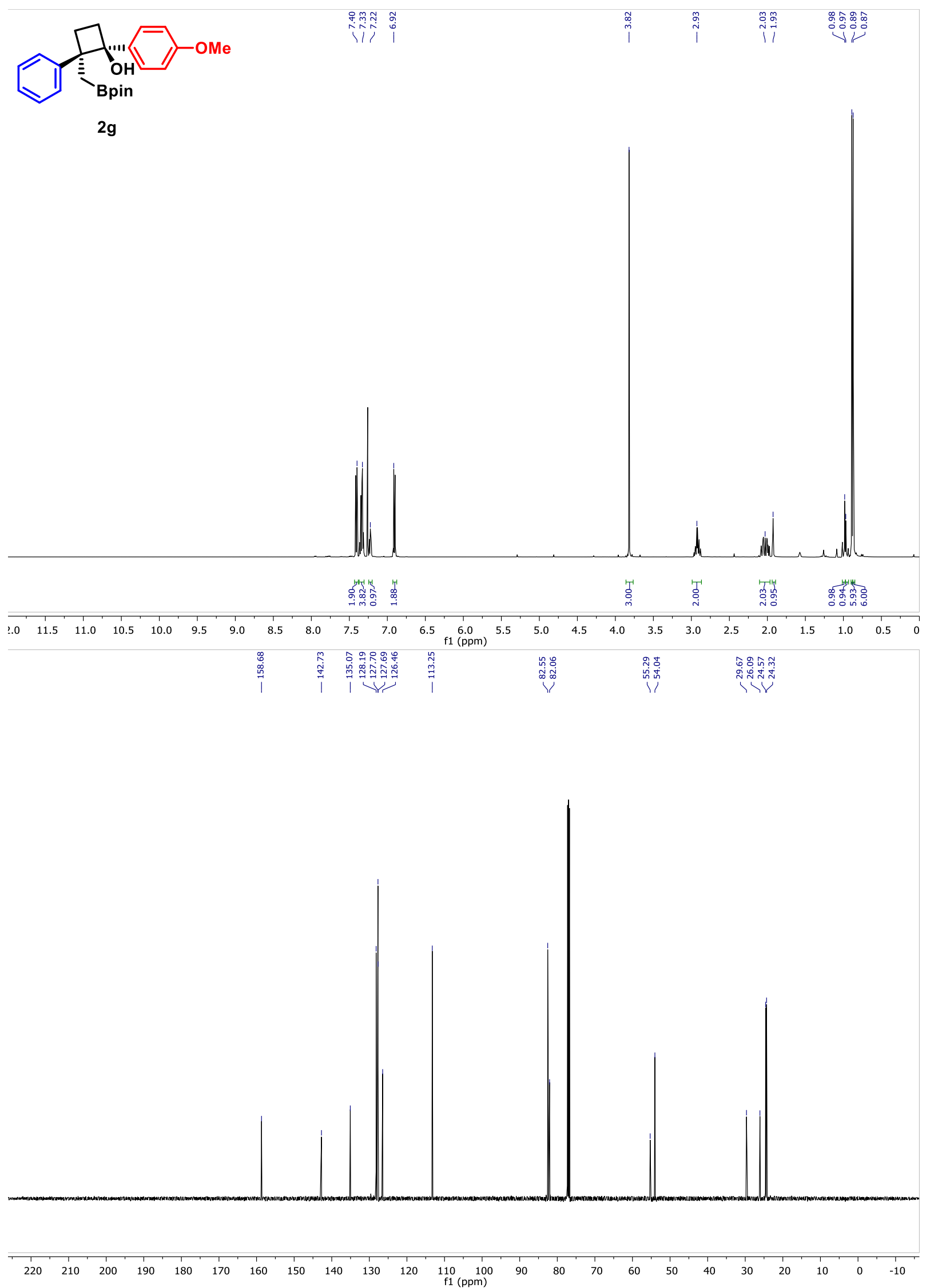


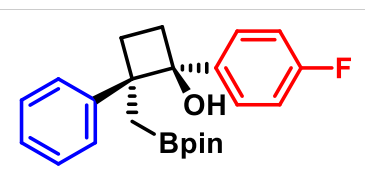

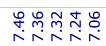

पा।

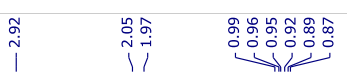

$2 \mathrm{~h}$
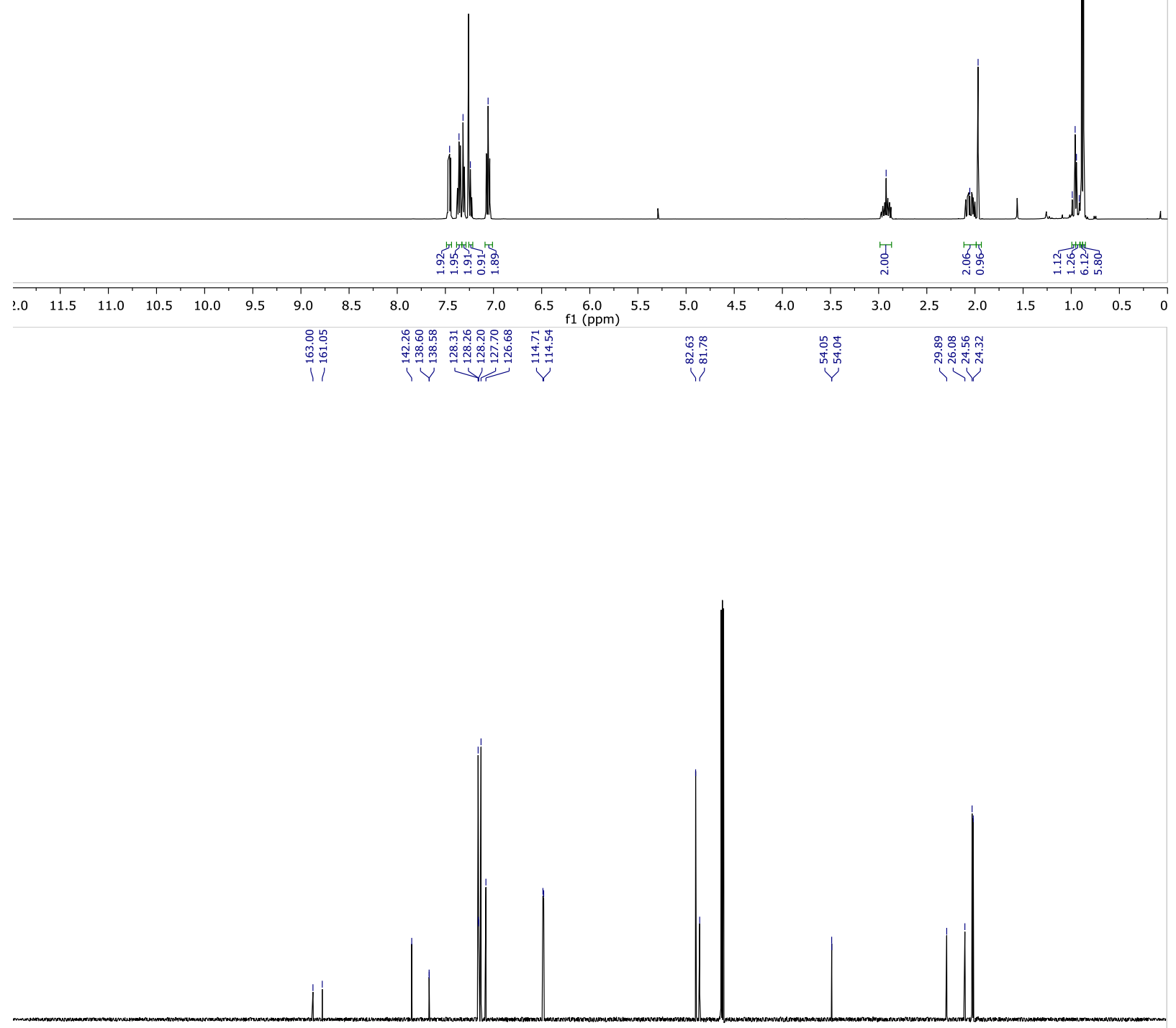

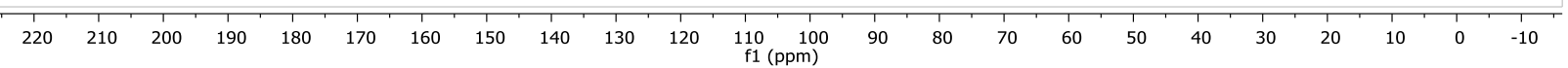




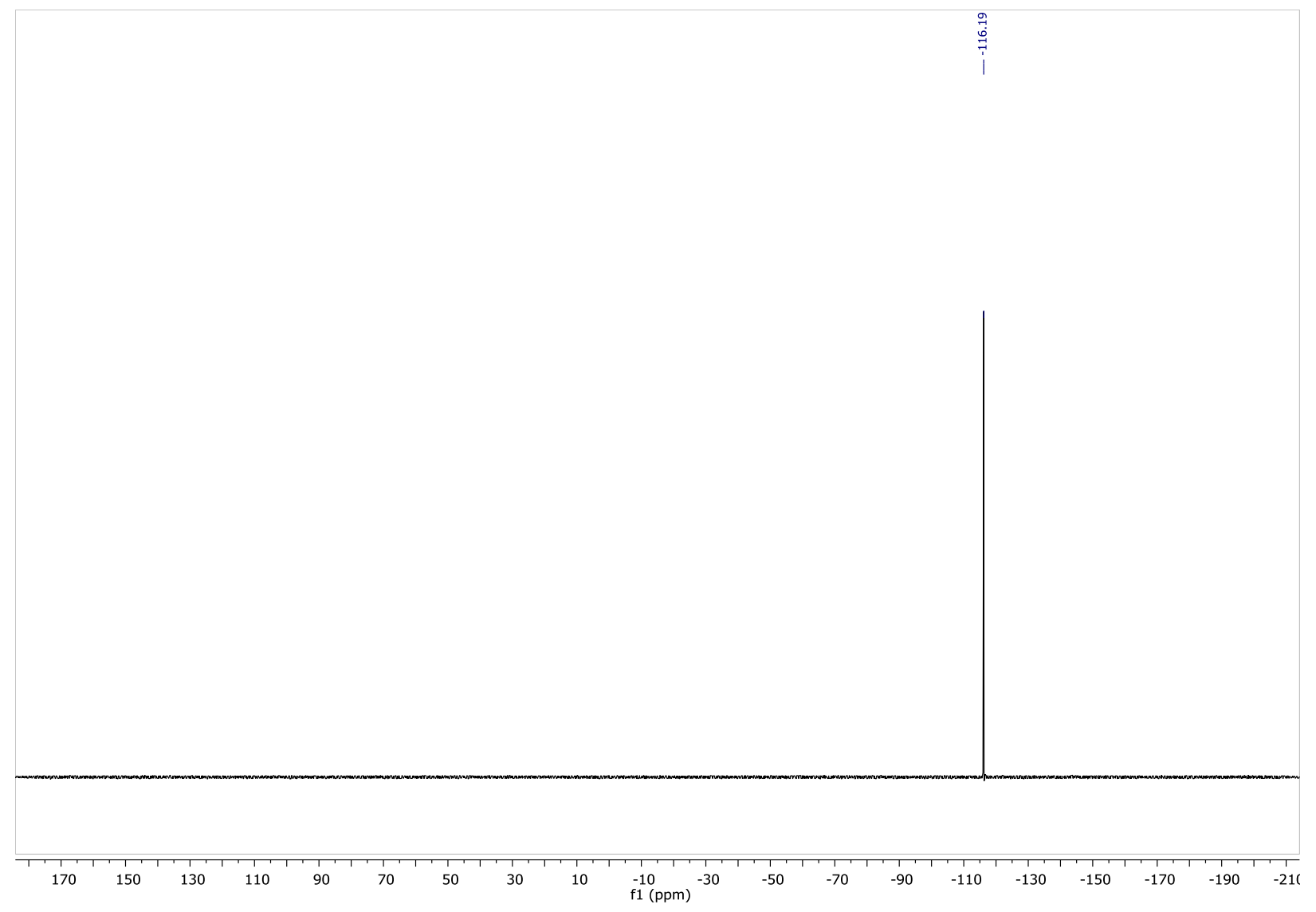



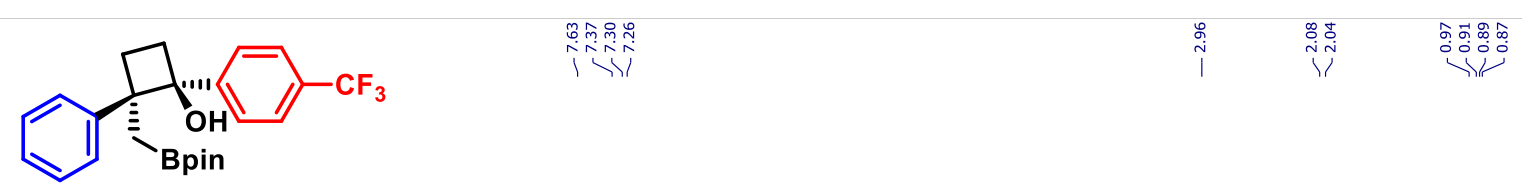

2i
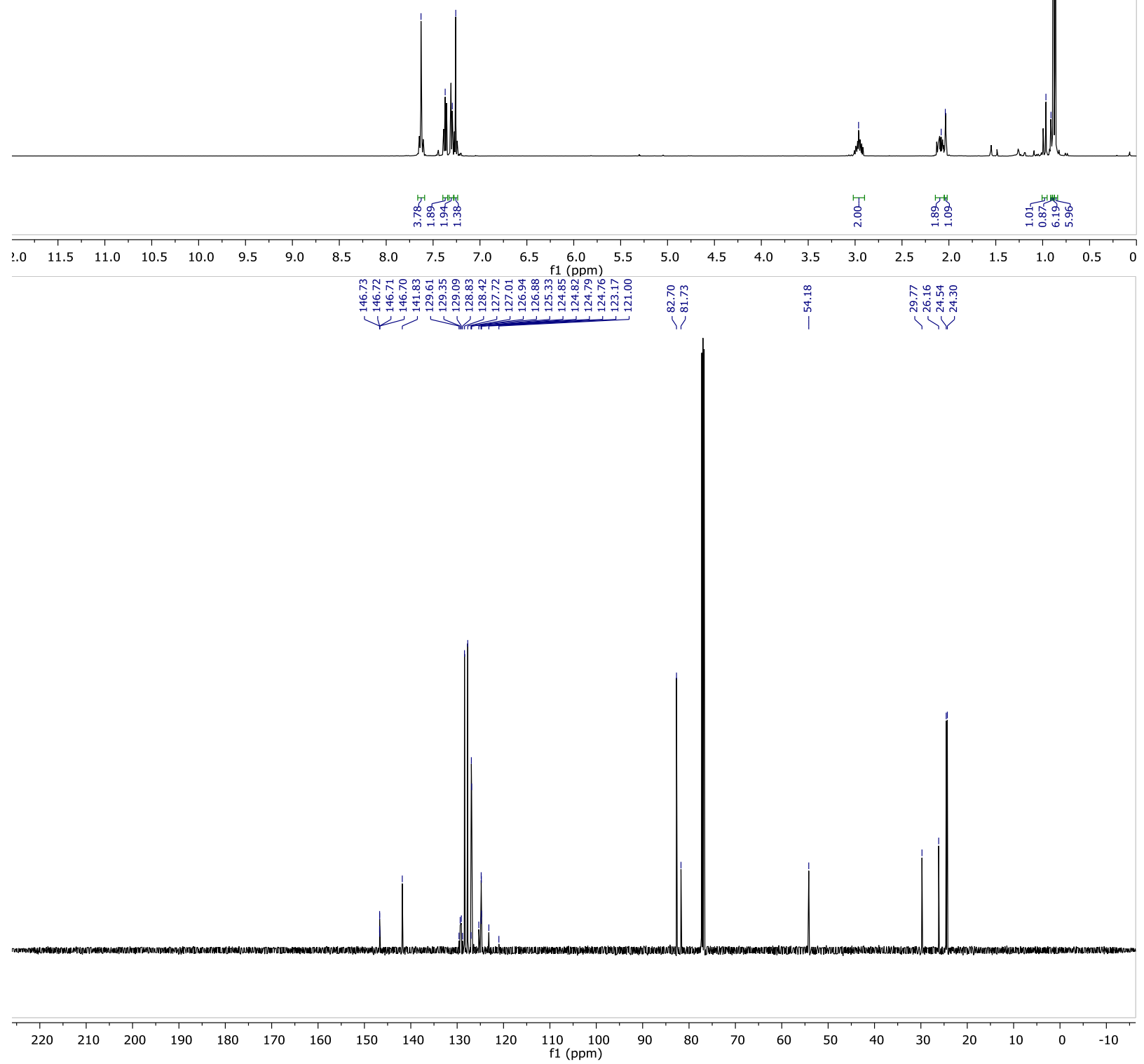


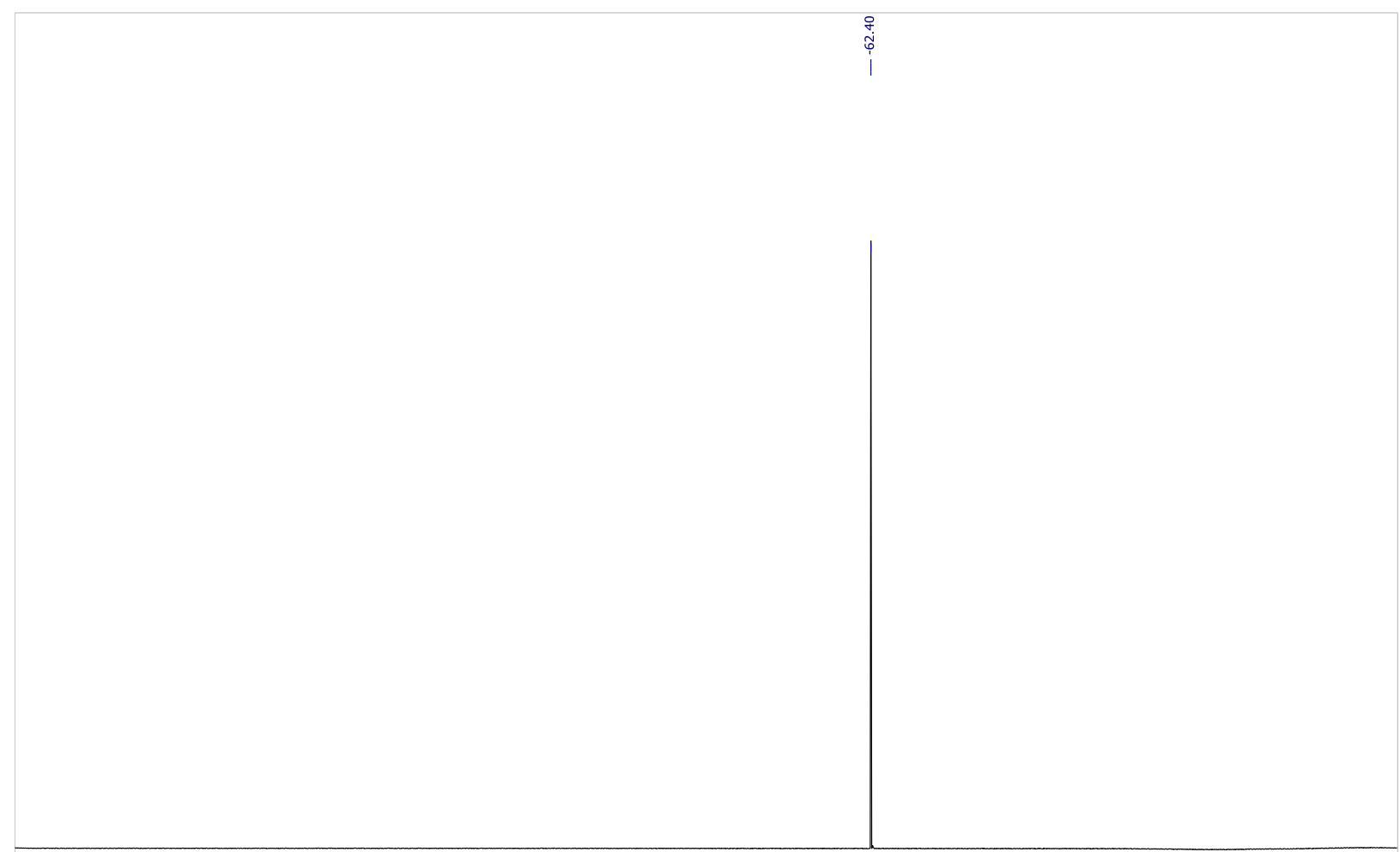

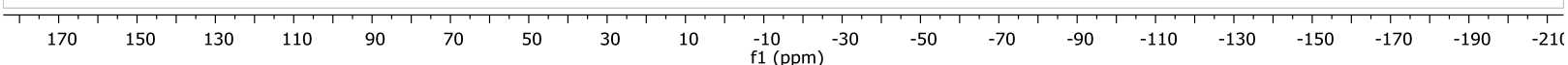




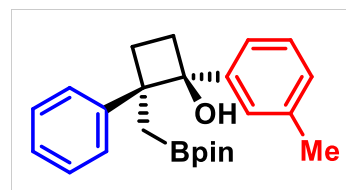

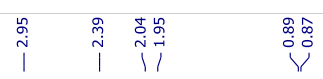

2j

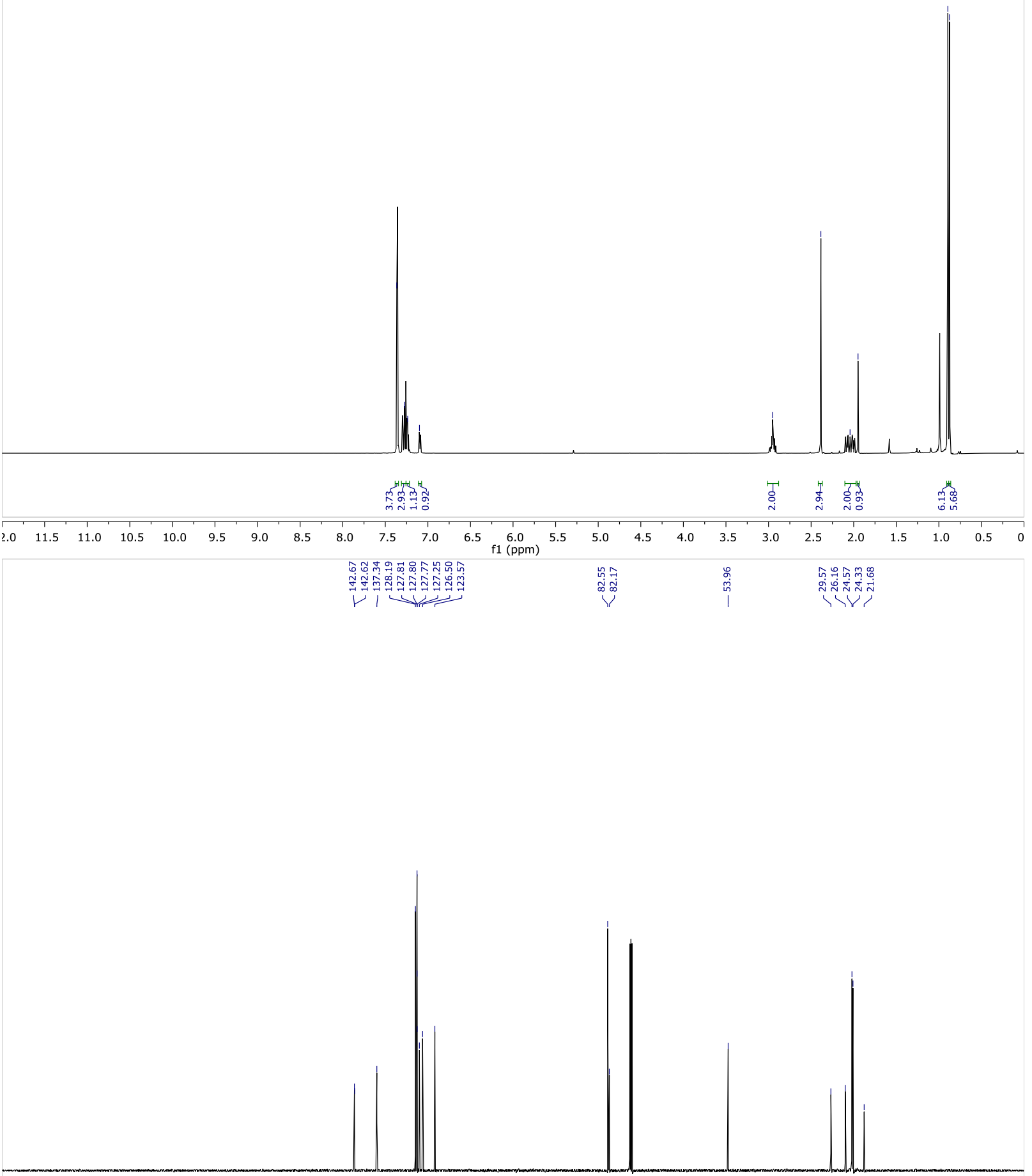

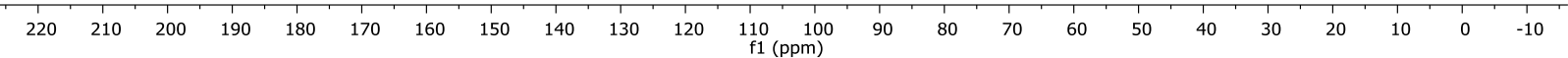



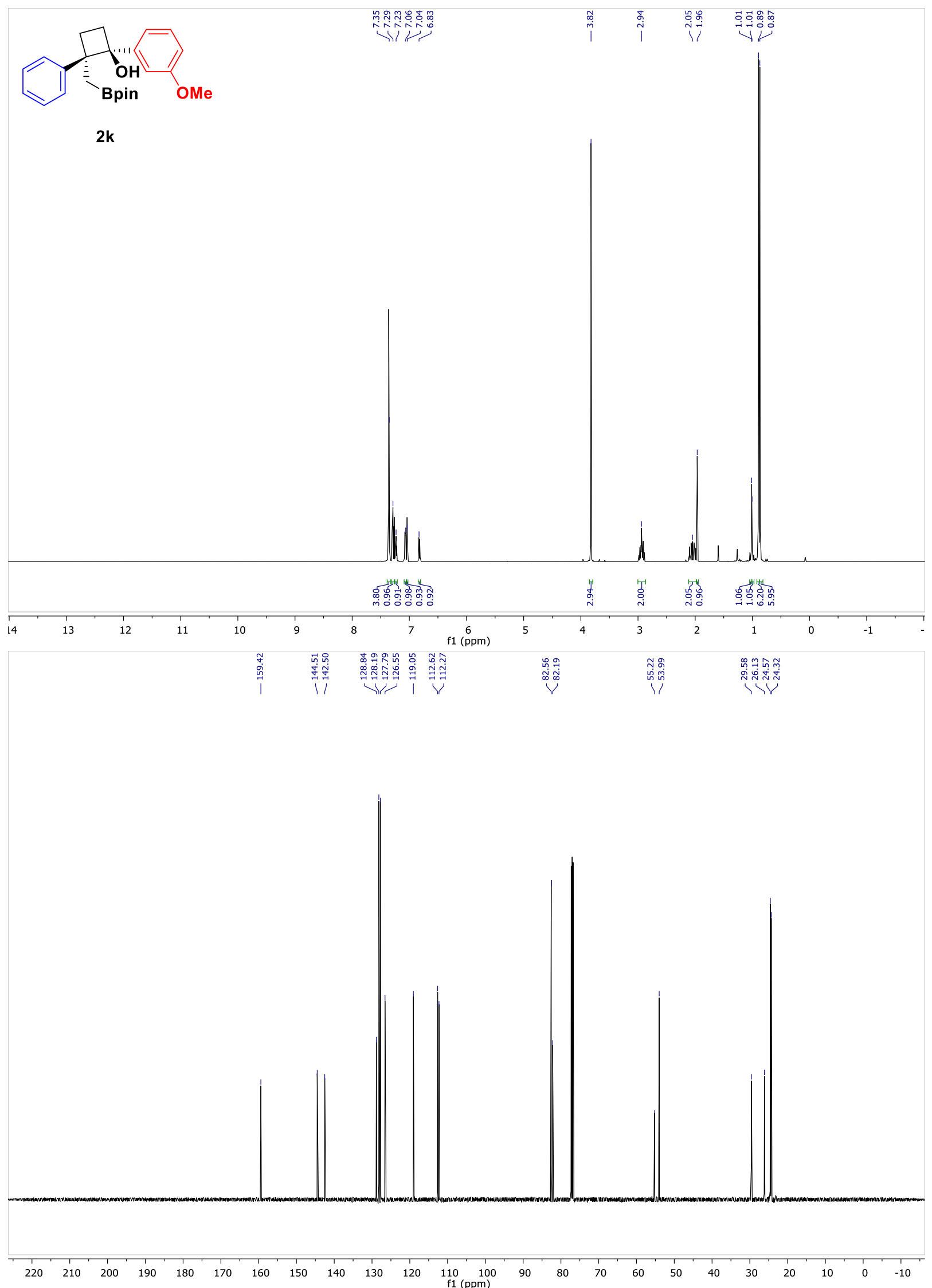


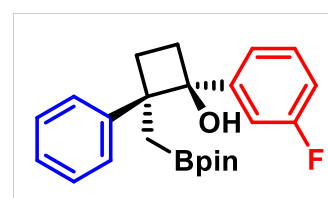

웃워숭

|ऐ।

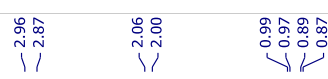

21
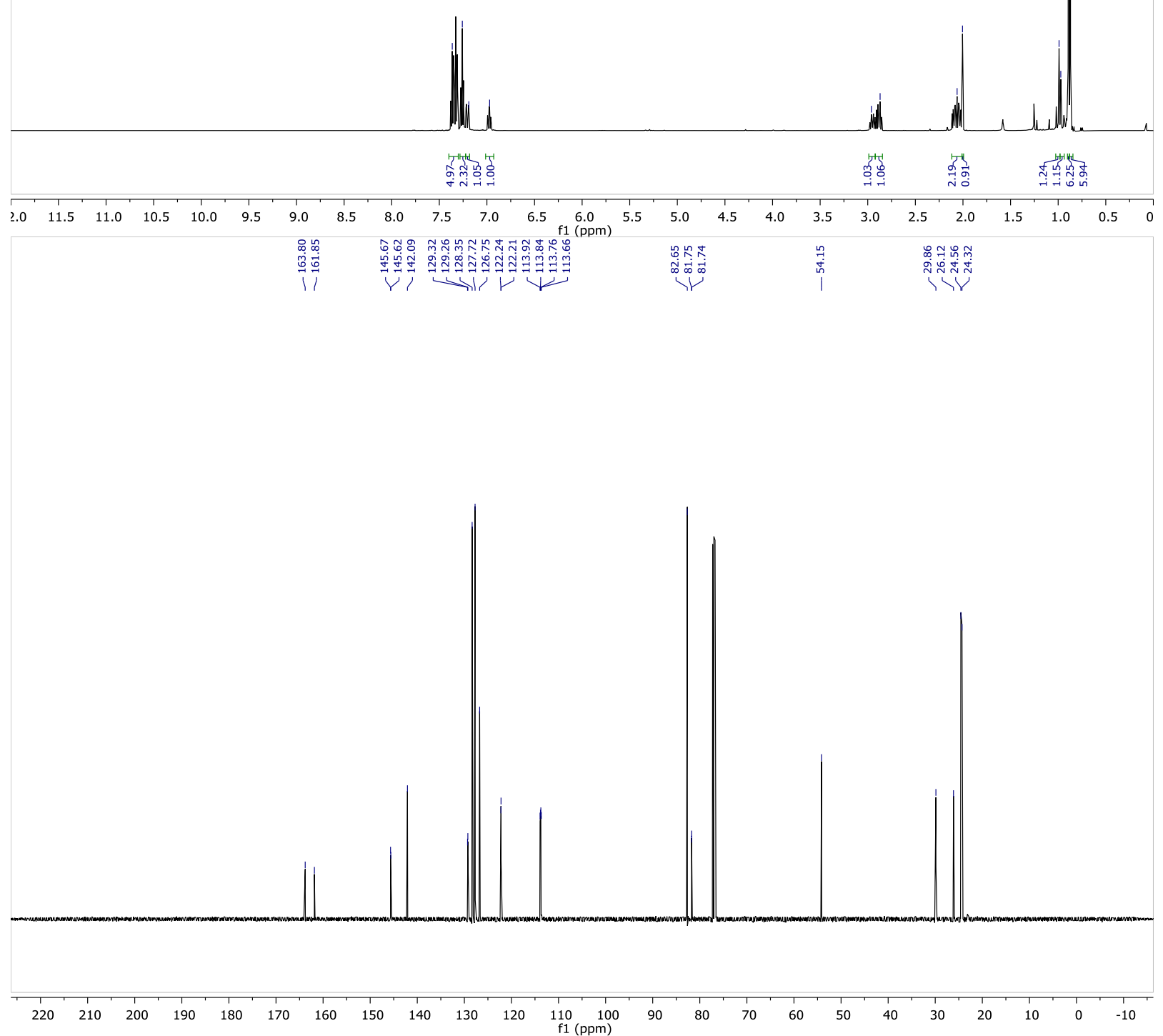


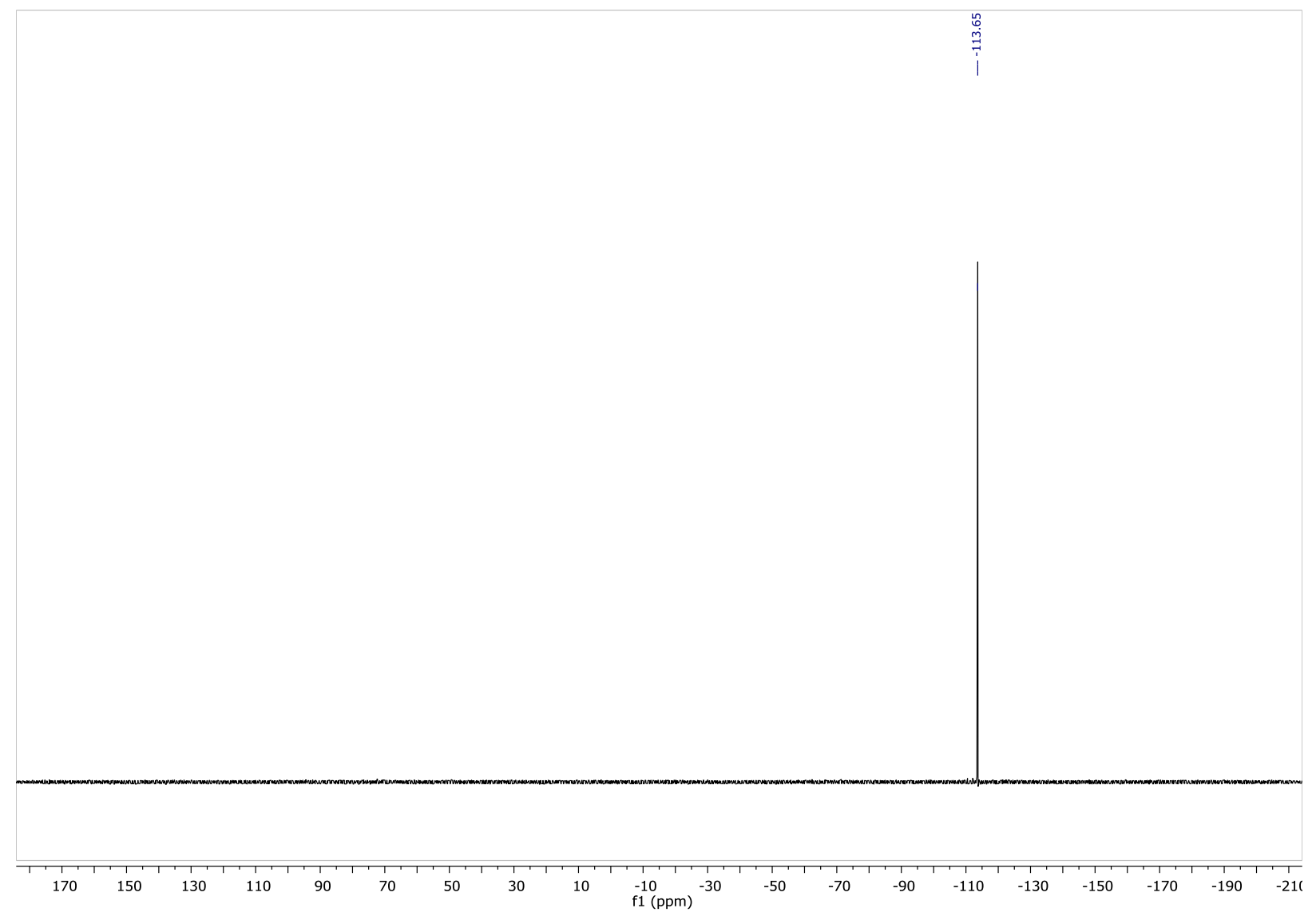



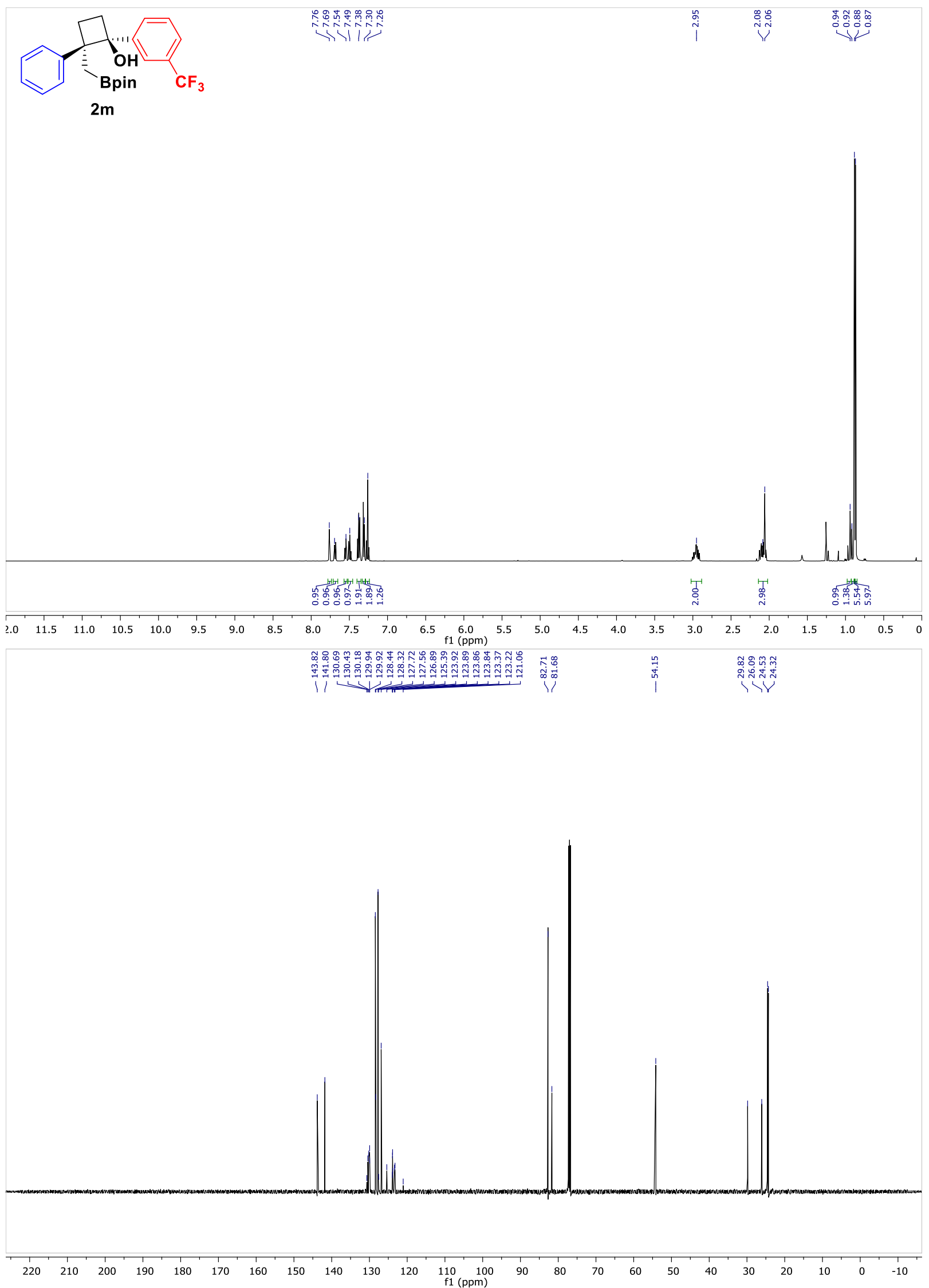


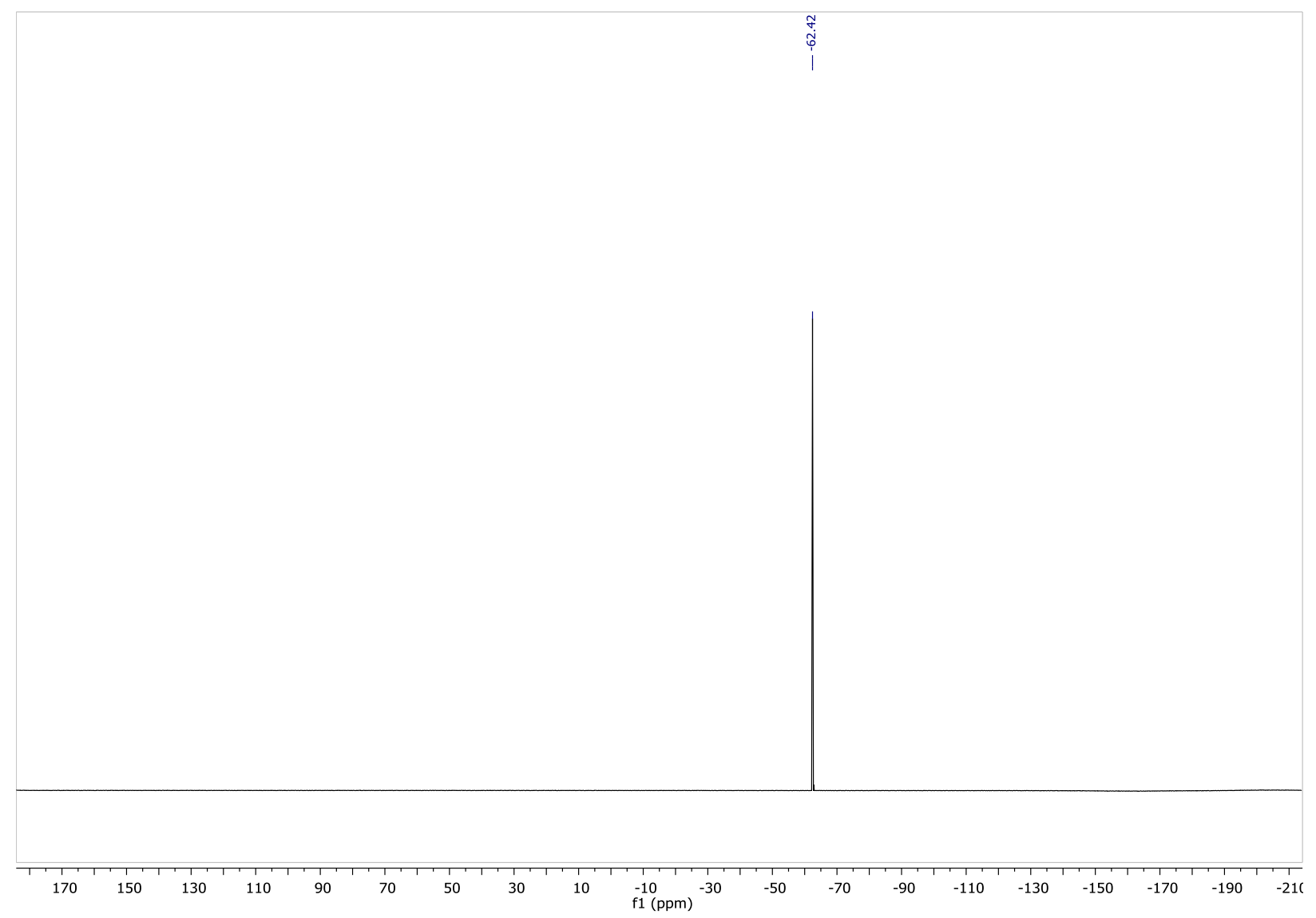




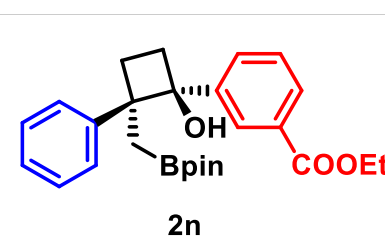

|

\section{$\stackrel{?}{+}$}

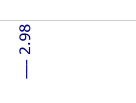

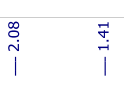
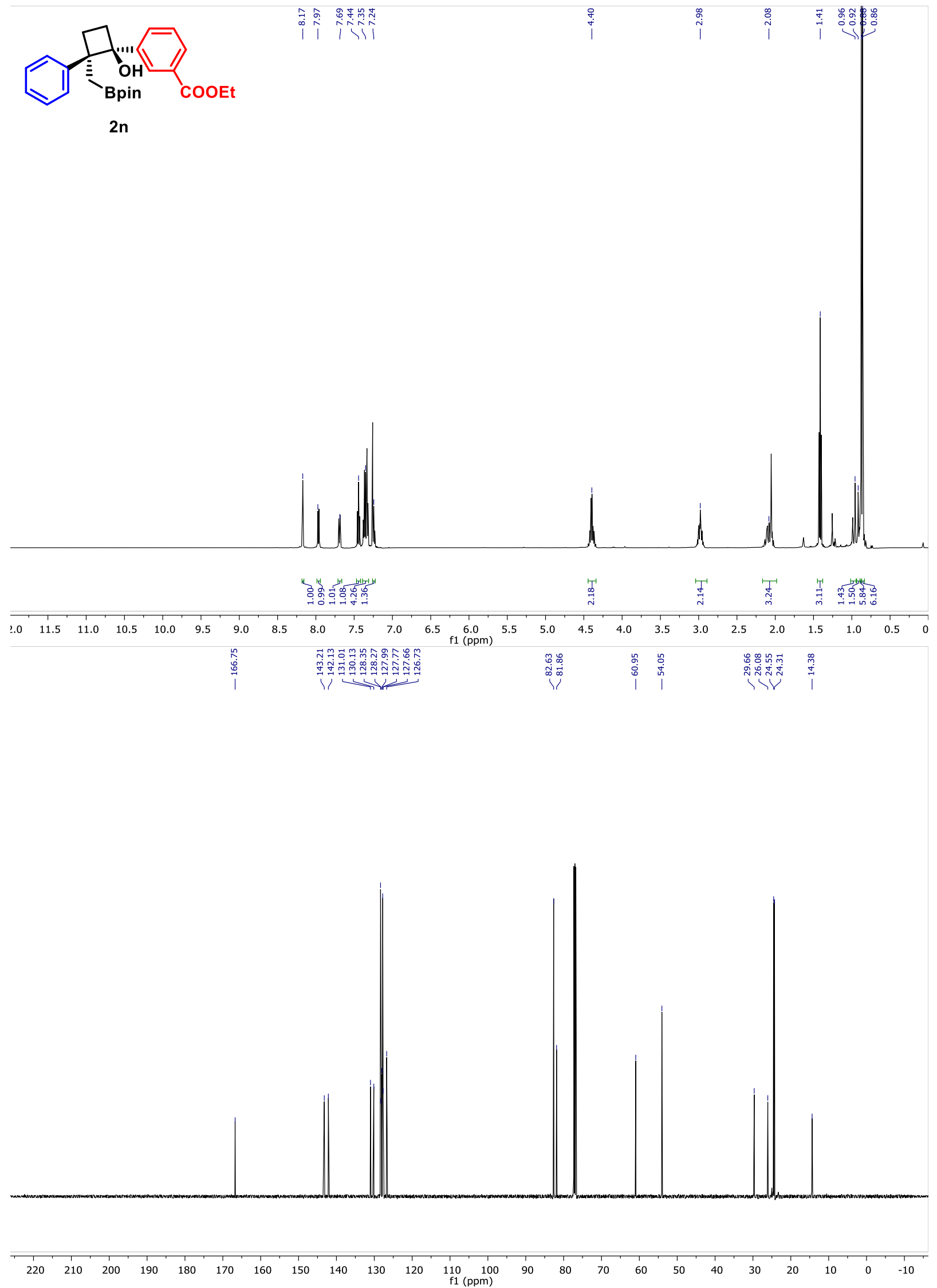


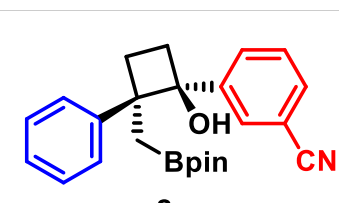

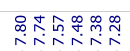

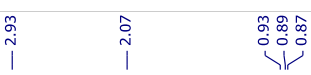

20
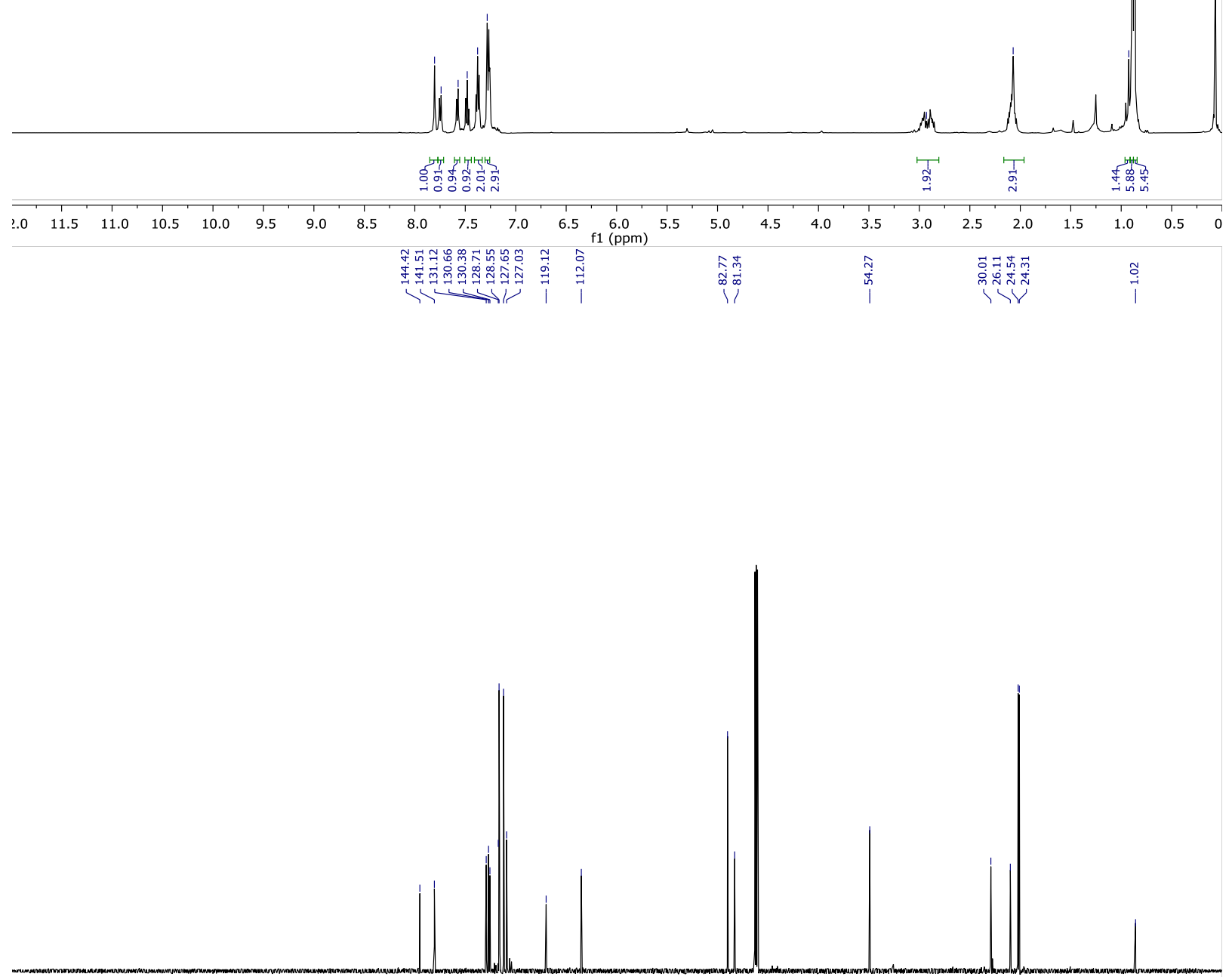

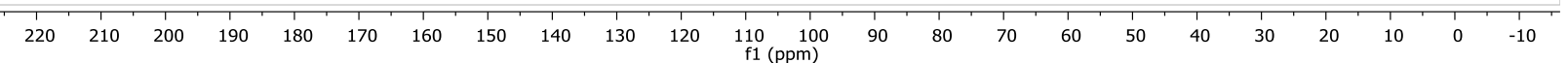



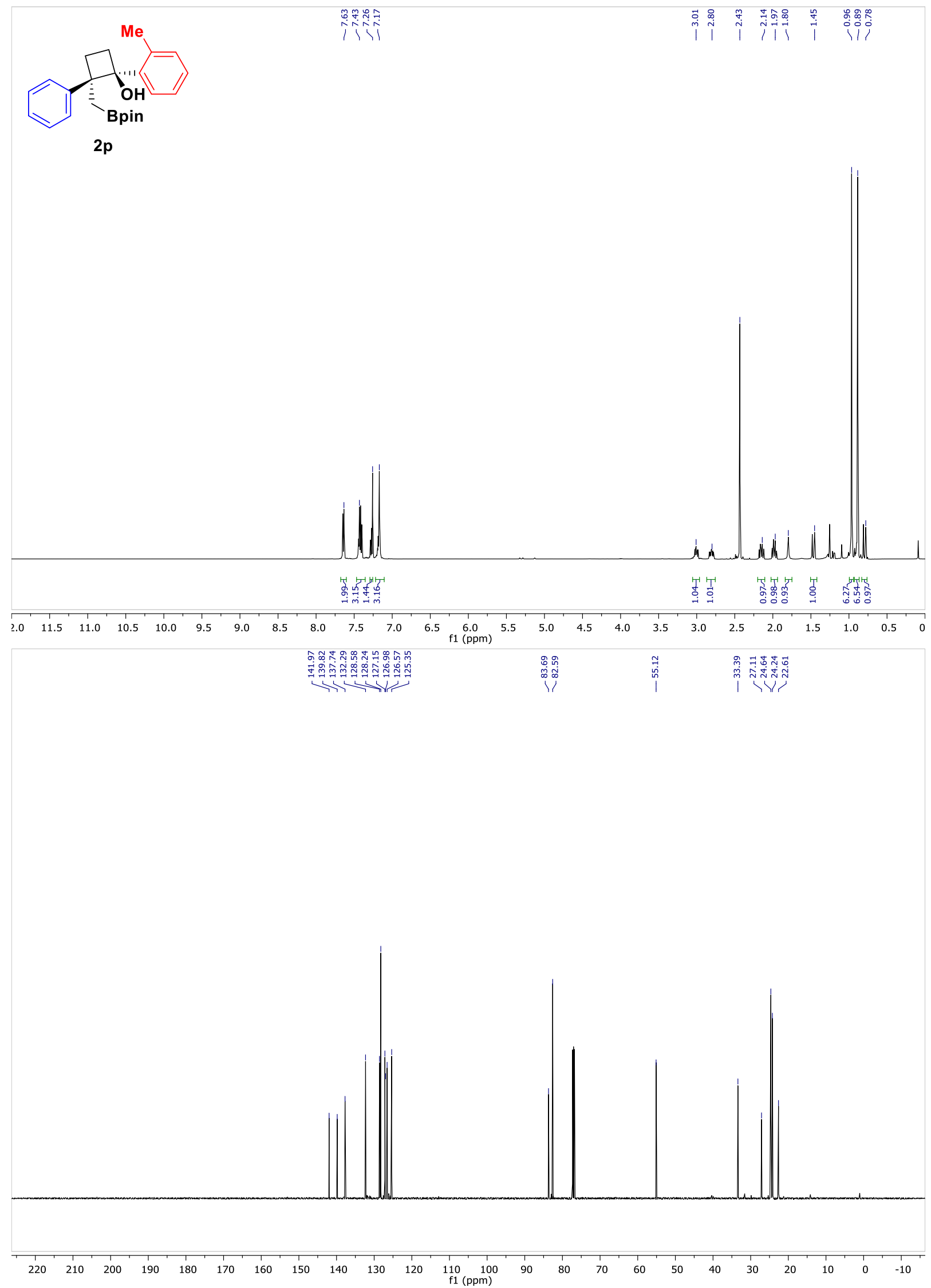


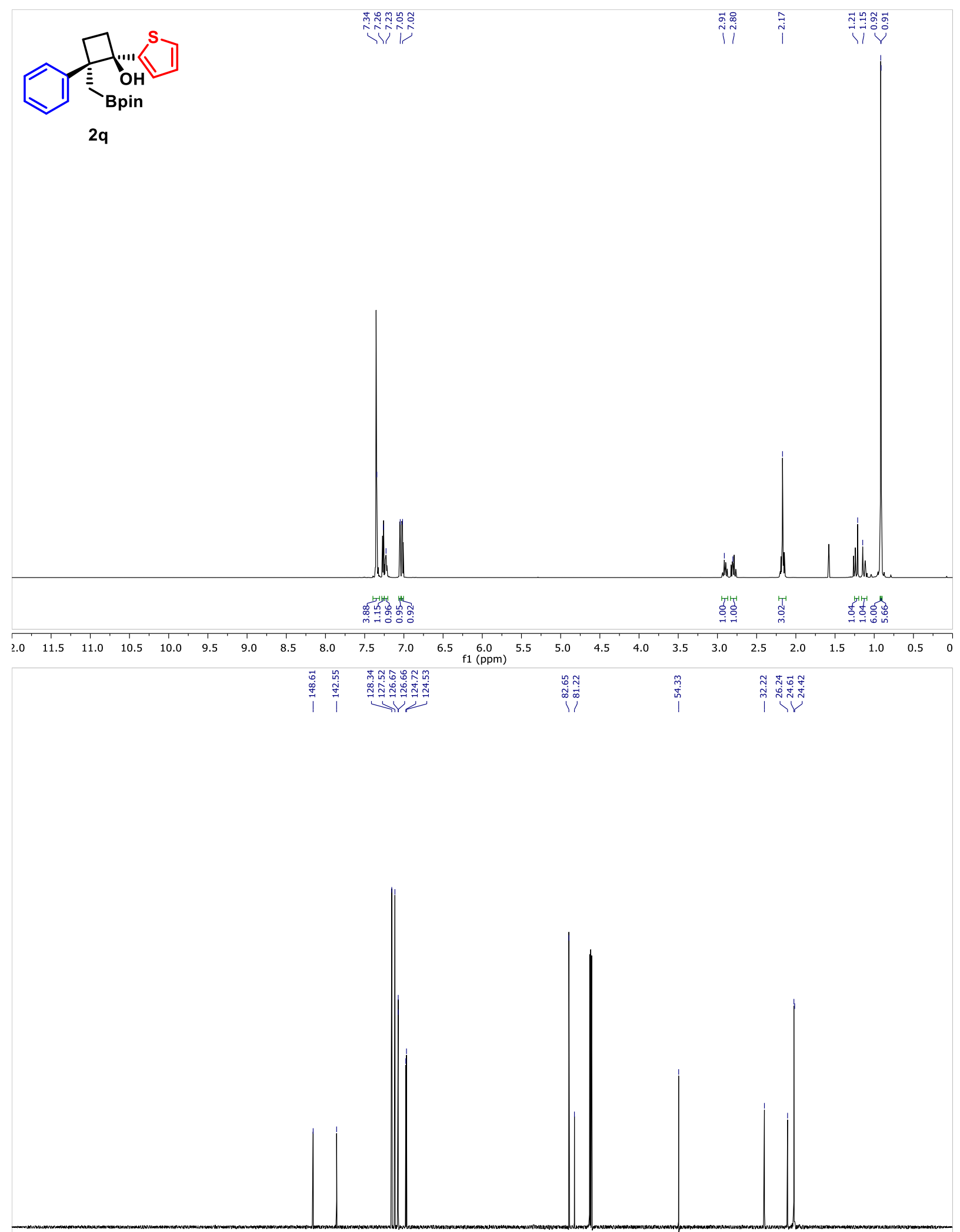

$\begin{array}{rllllllllllllllllllllllllll}220 & 210 & 200 & 190 & 180 & 170 & 160 & 150 & 140 & 130 & 120 & 110 & 100 & 90 & 80 & 70 & 60 & 50 & 40 & 30 & 20 & 10 & 0 & -10\end{array}$ 

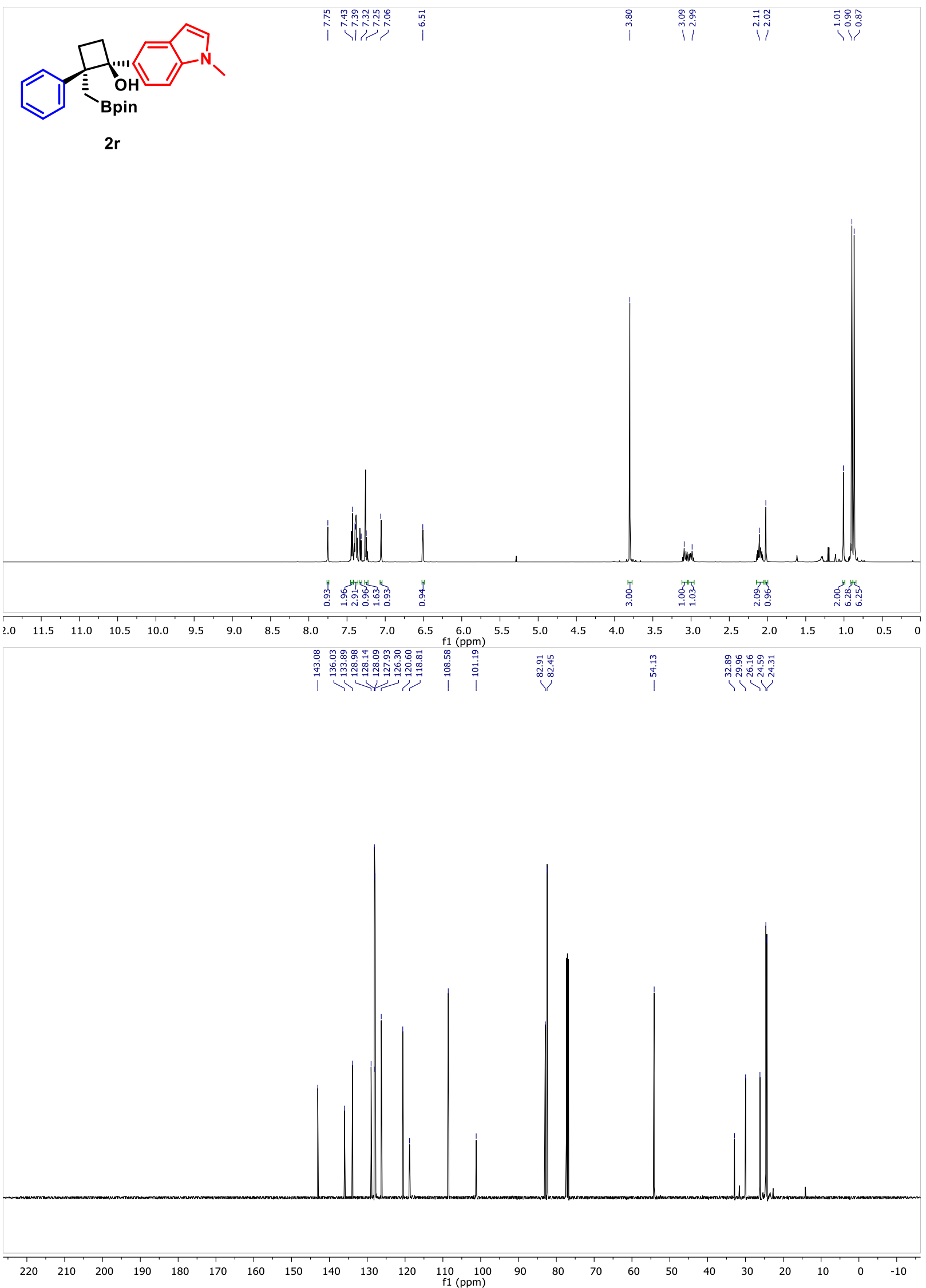

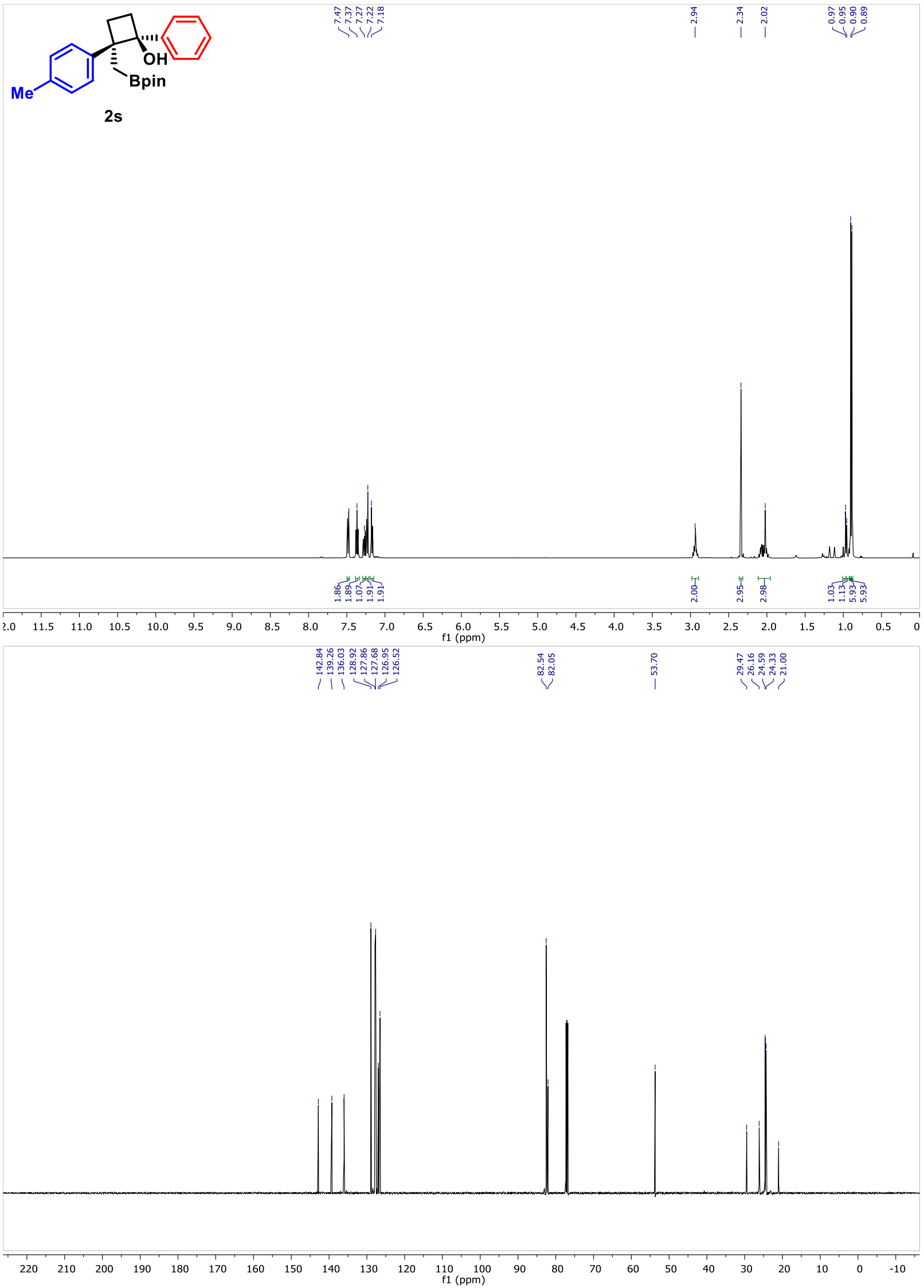

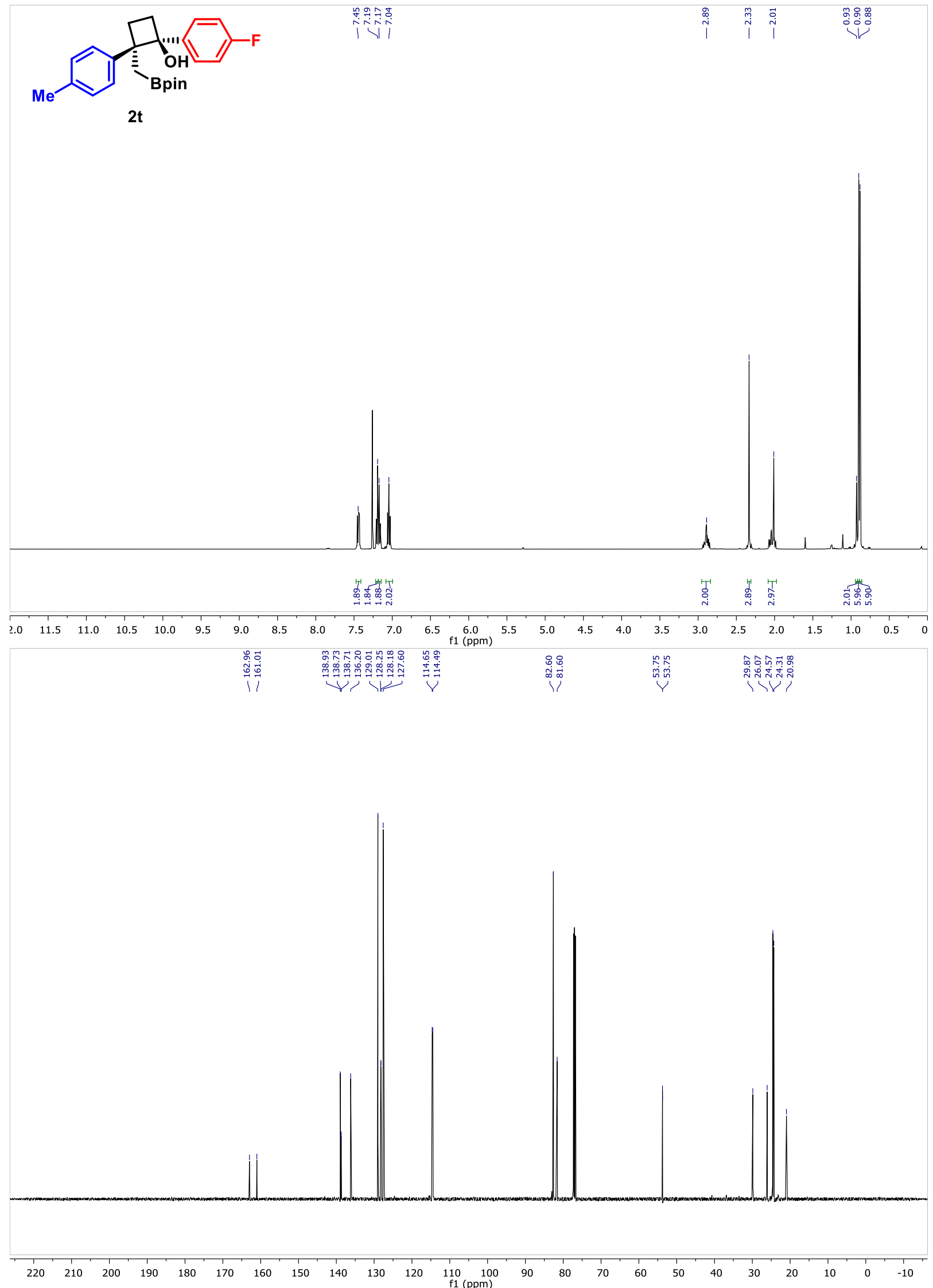


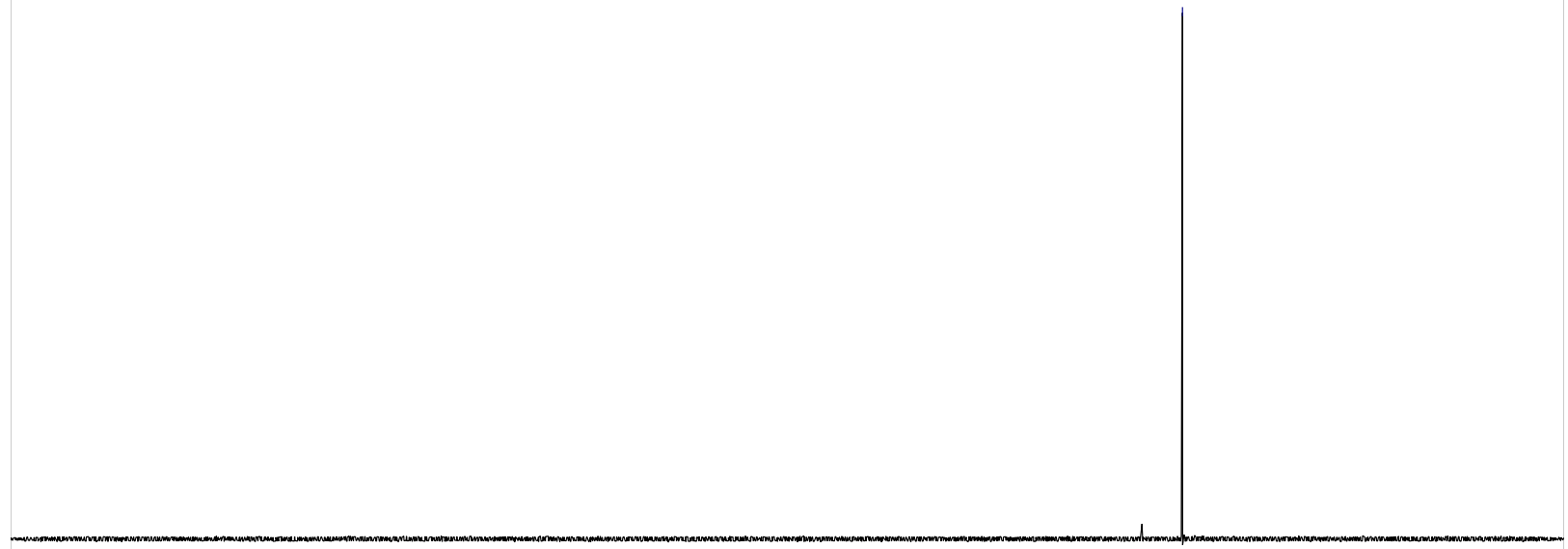

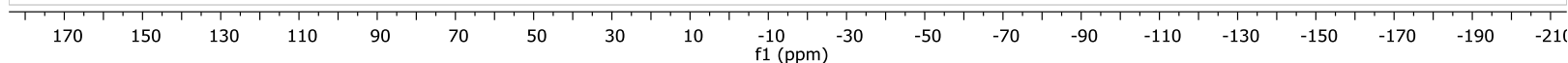



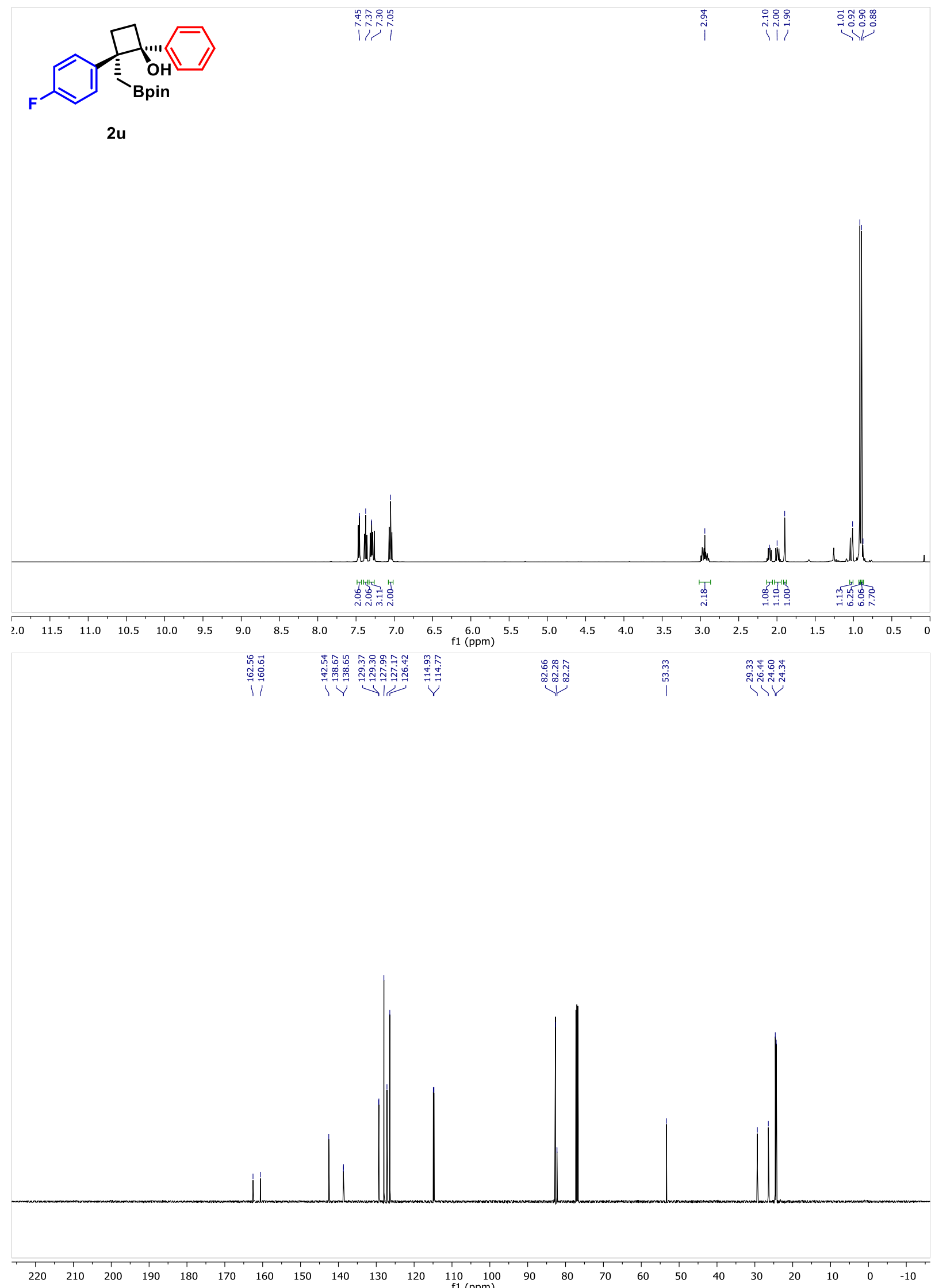


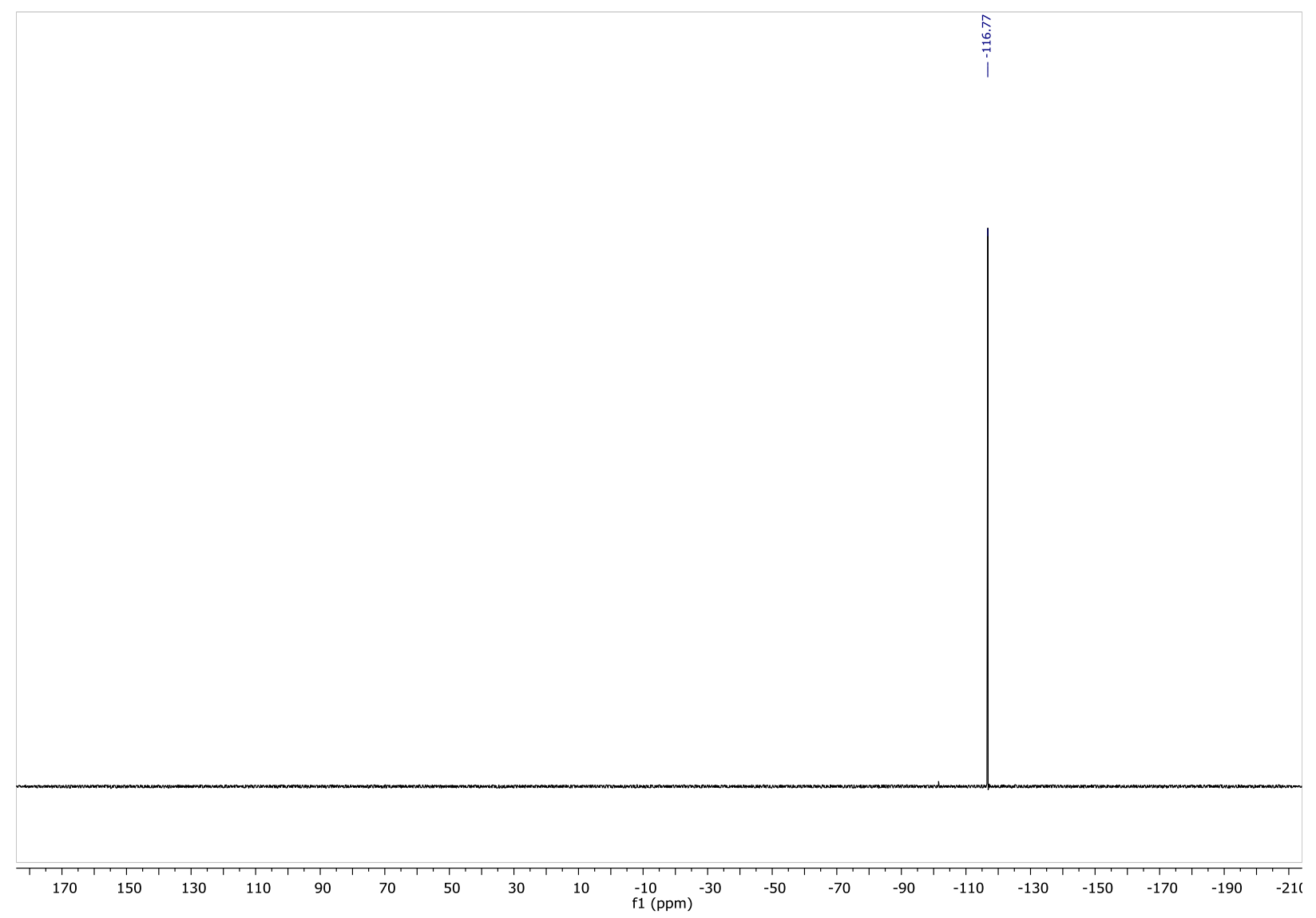




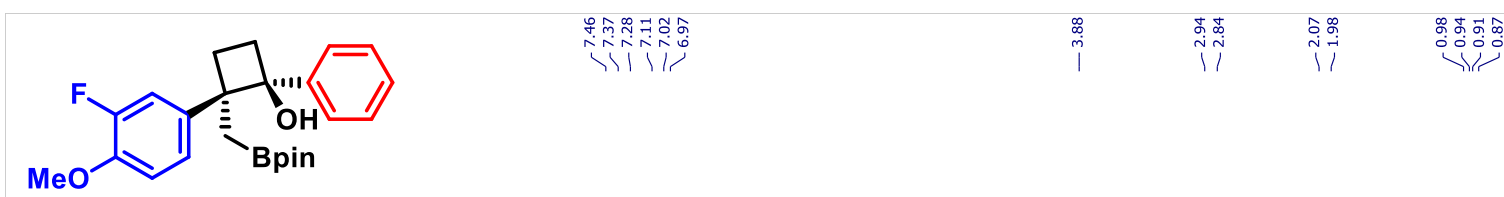

2v
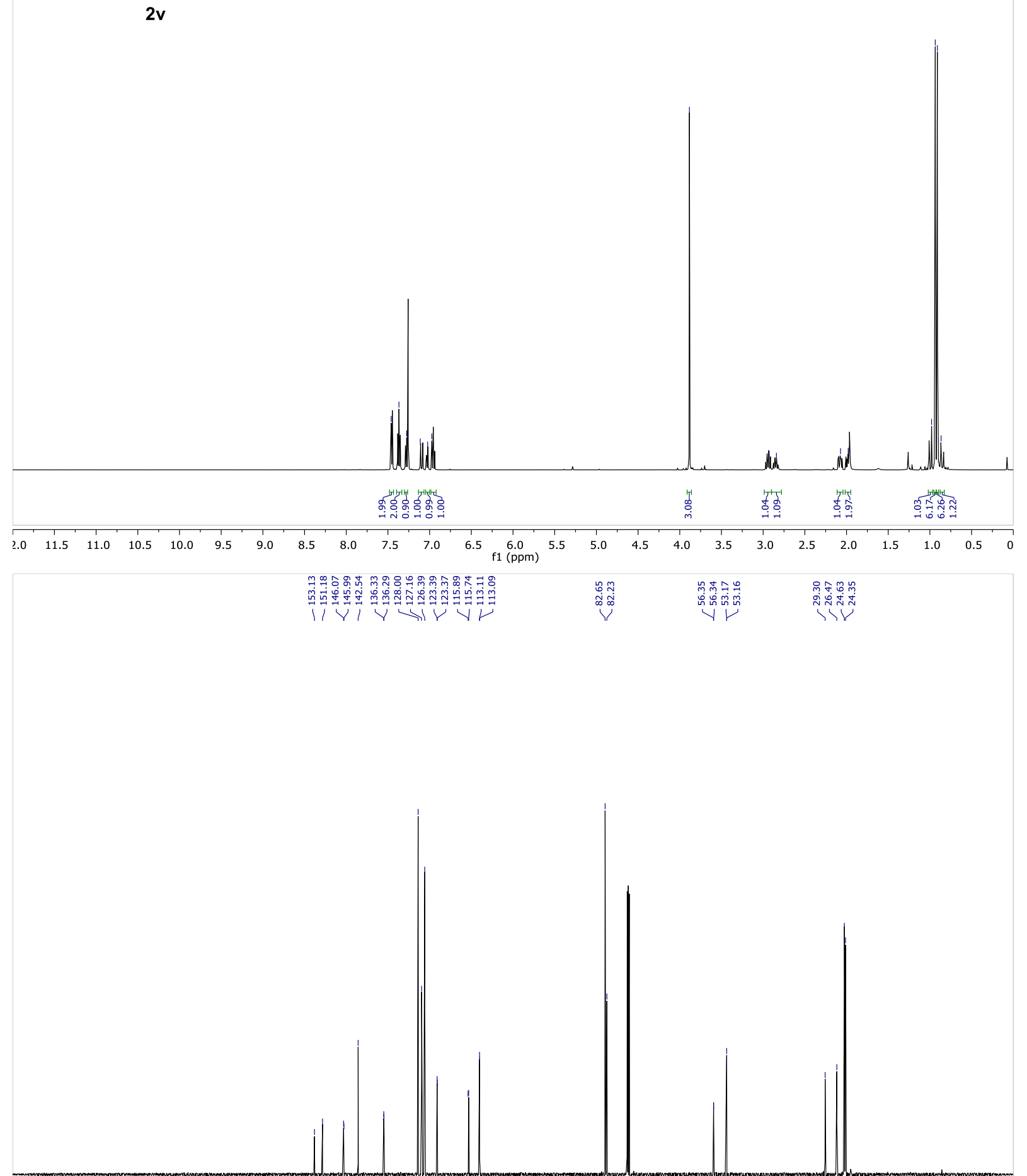

$\begin{array}{llllllllllllllllllllllll}220 & 210 & 200 & 190 & 180 & 170 & 160 & 150 & 140 & 130 & 120 & \begin{array}{c}110 \\ \mathrm{f} 1(\mathrm{ppm})\end{array} & 90 & 80 & 70 & 60 & 50 & 40 & 30 & 20 & 10 & 0 & -10\end{array}$ 


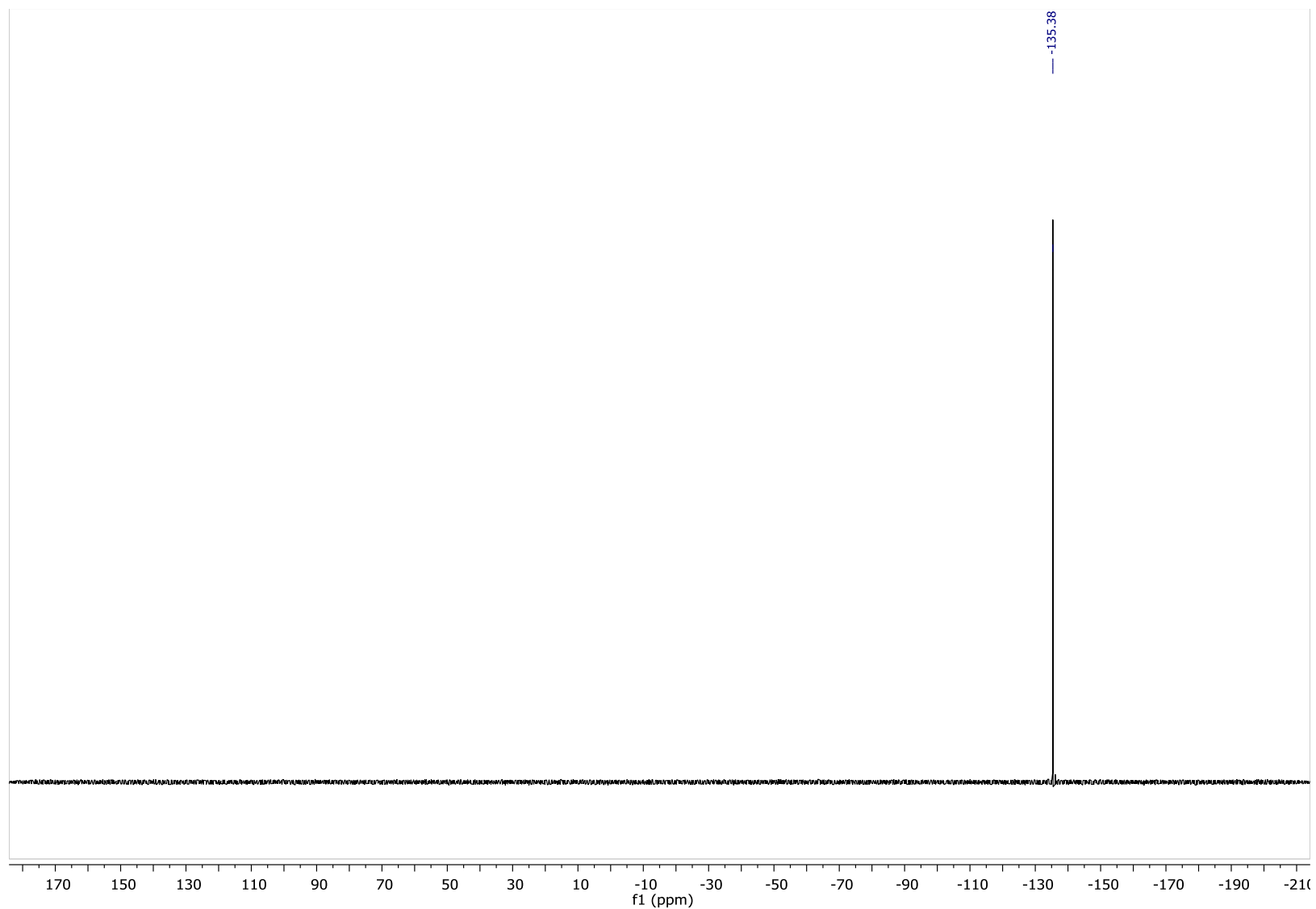




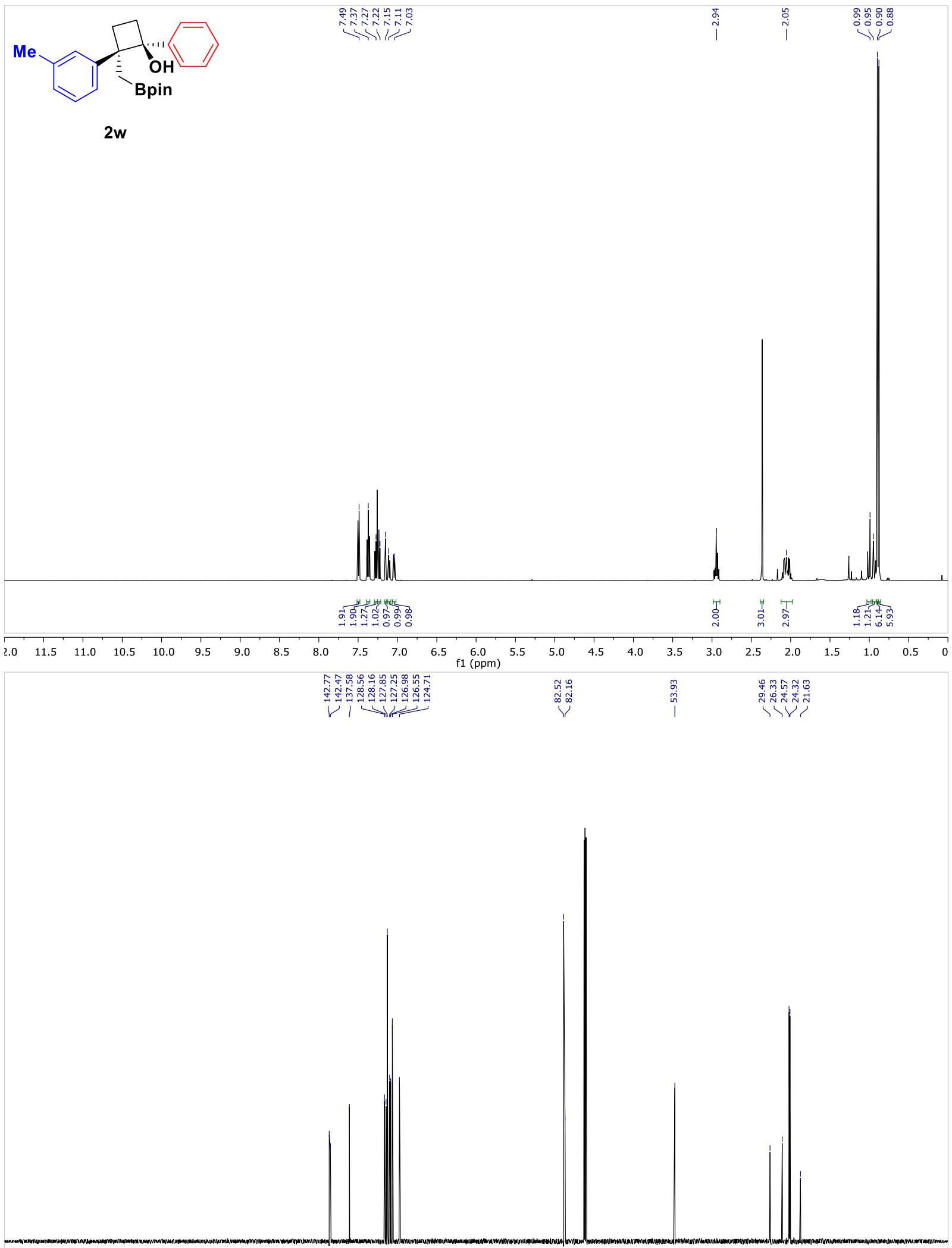

$\begin{array}{lllllllllllllllllllllllllllllll}220 & 210 & 200 & 190 & 180 & 170 & 160 & 150 & 140 & 130 & 120 & 110 & 100 & 90 & 80 & 70 & 60 & 50 & 40 & 30 & 20 & 10 & 0 & -10\end{array}$ 

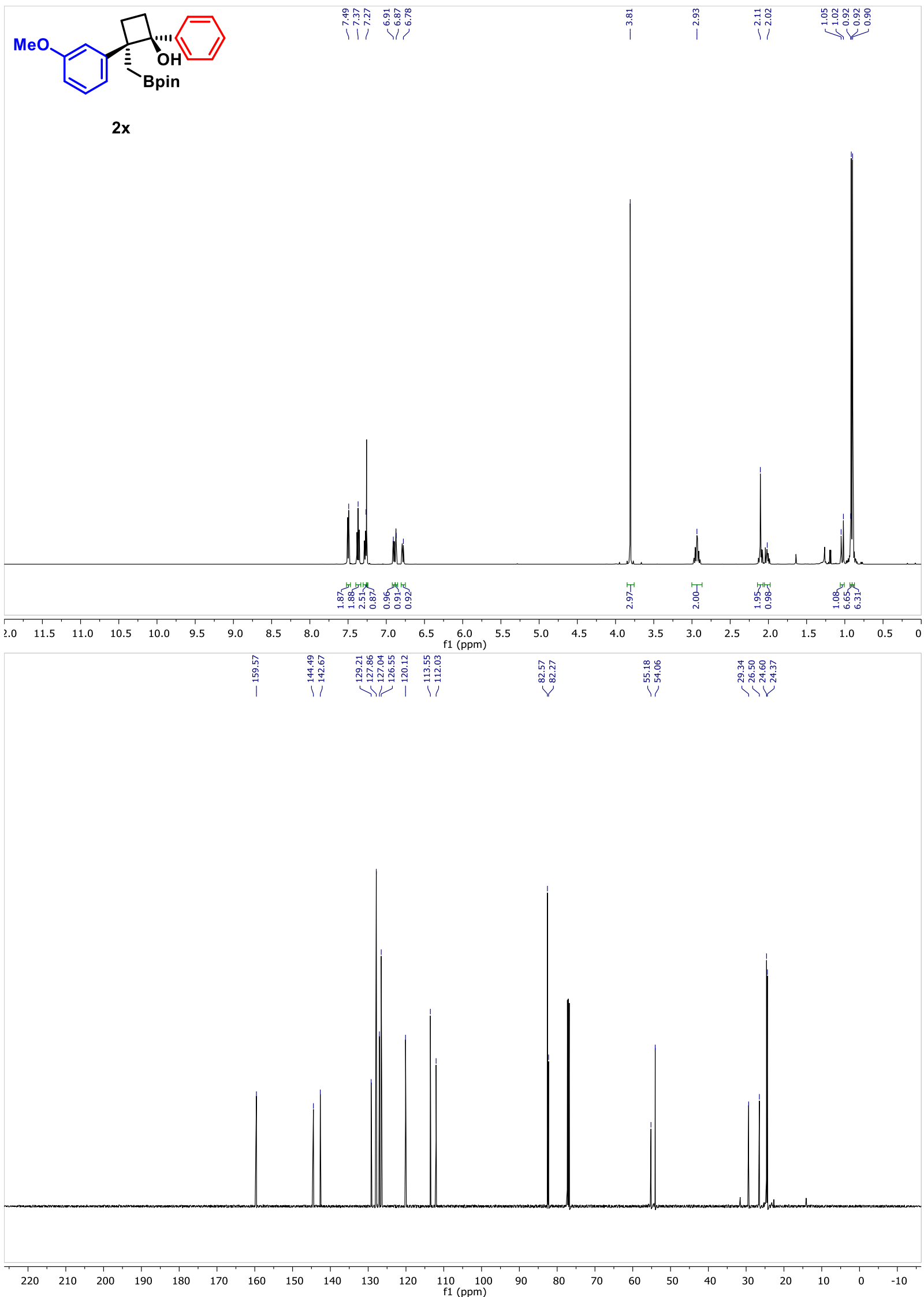


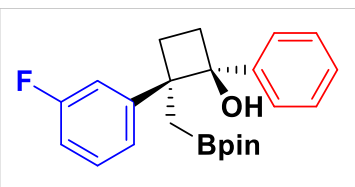

$2 y$
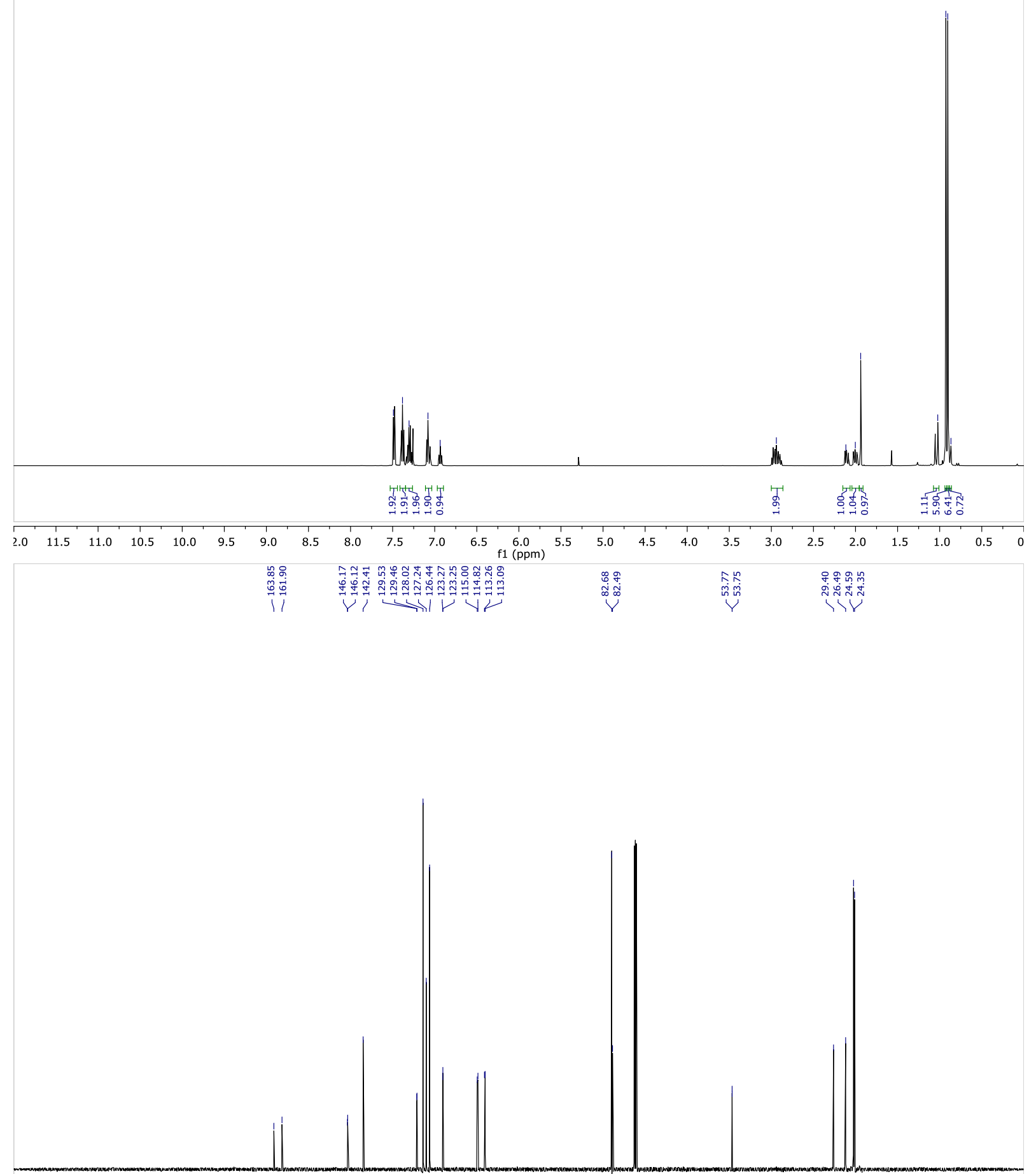

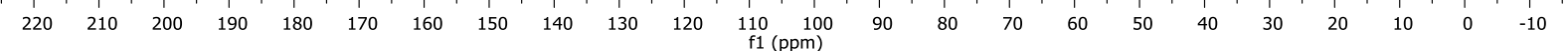




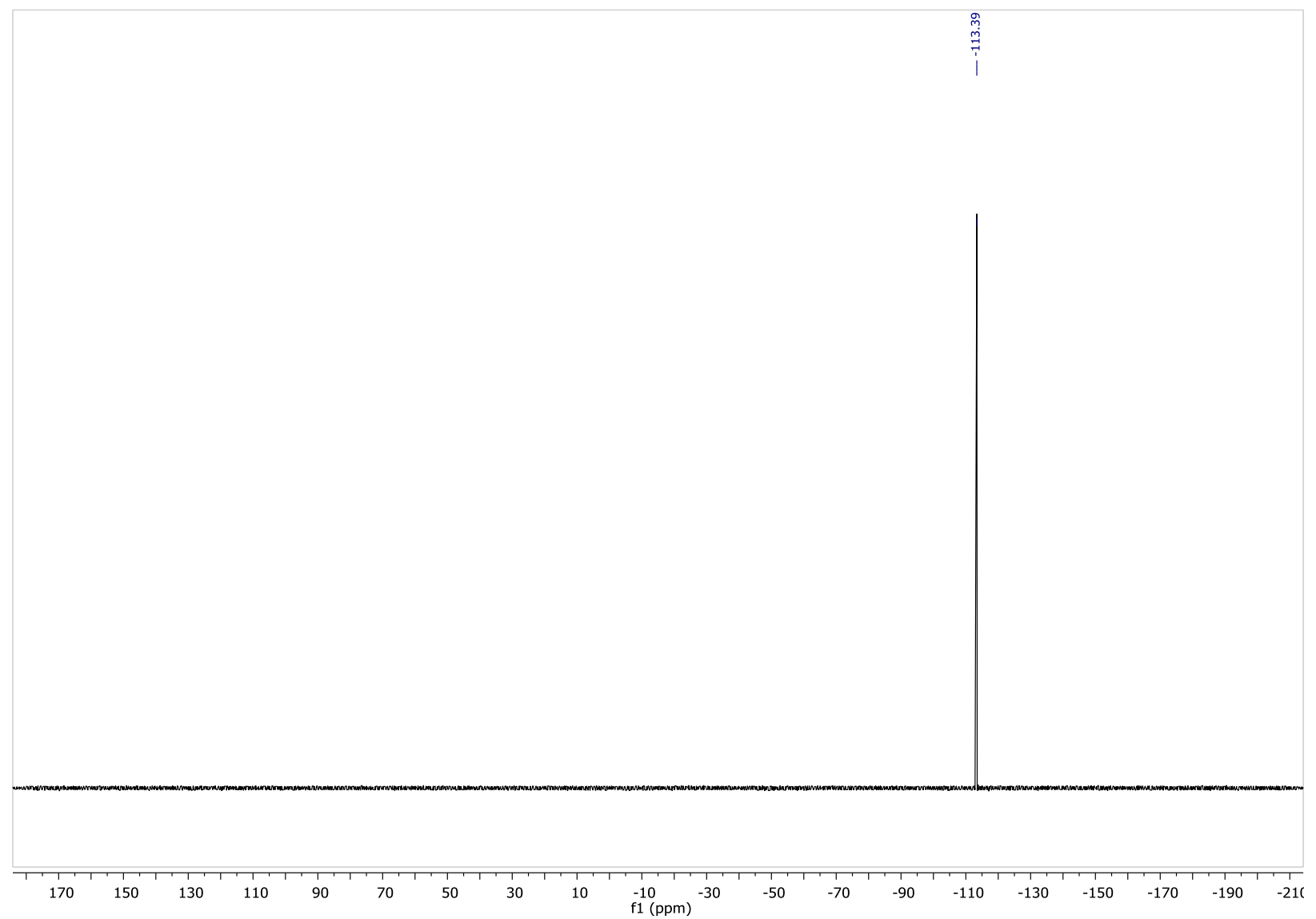



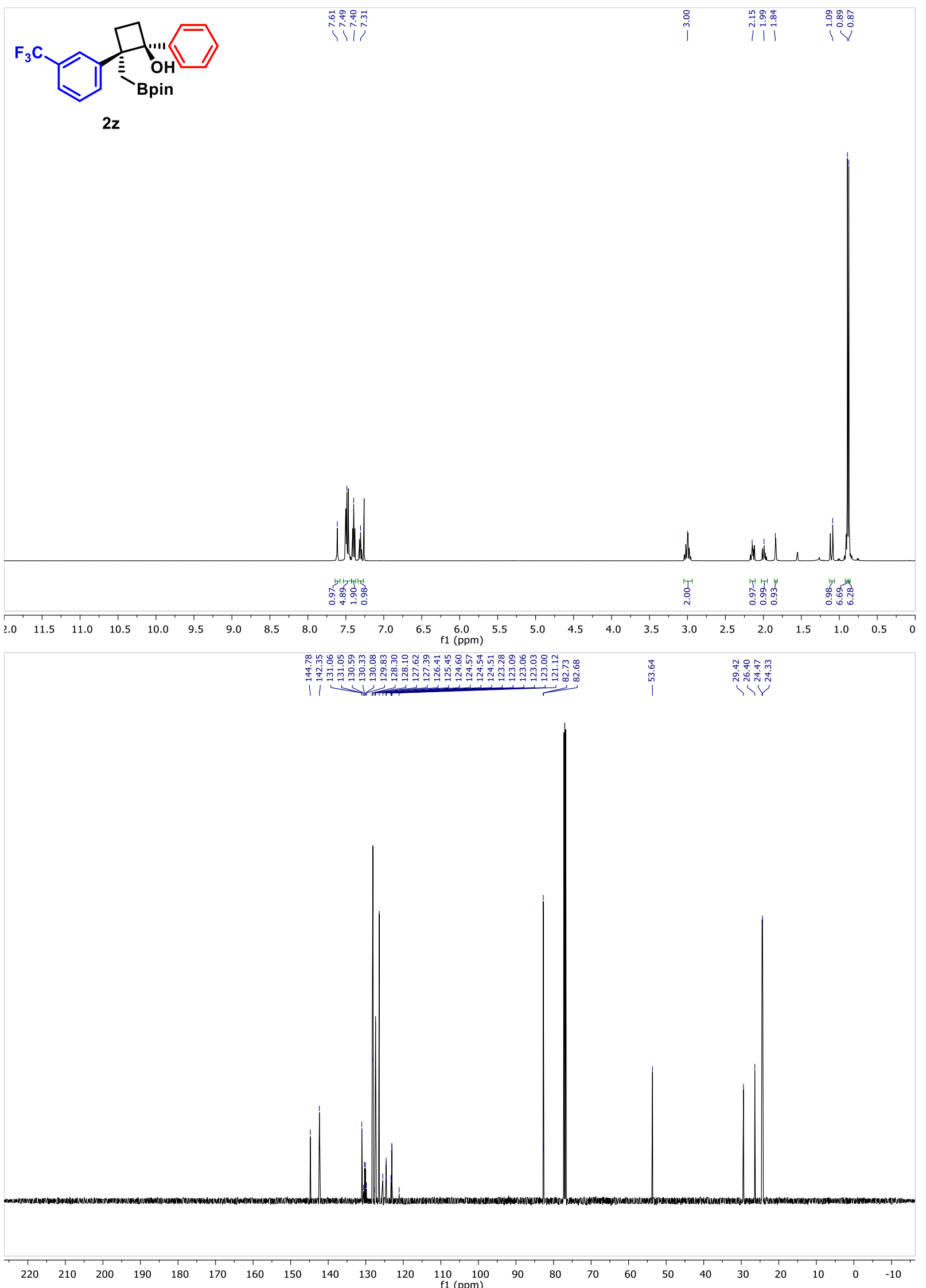


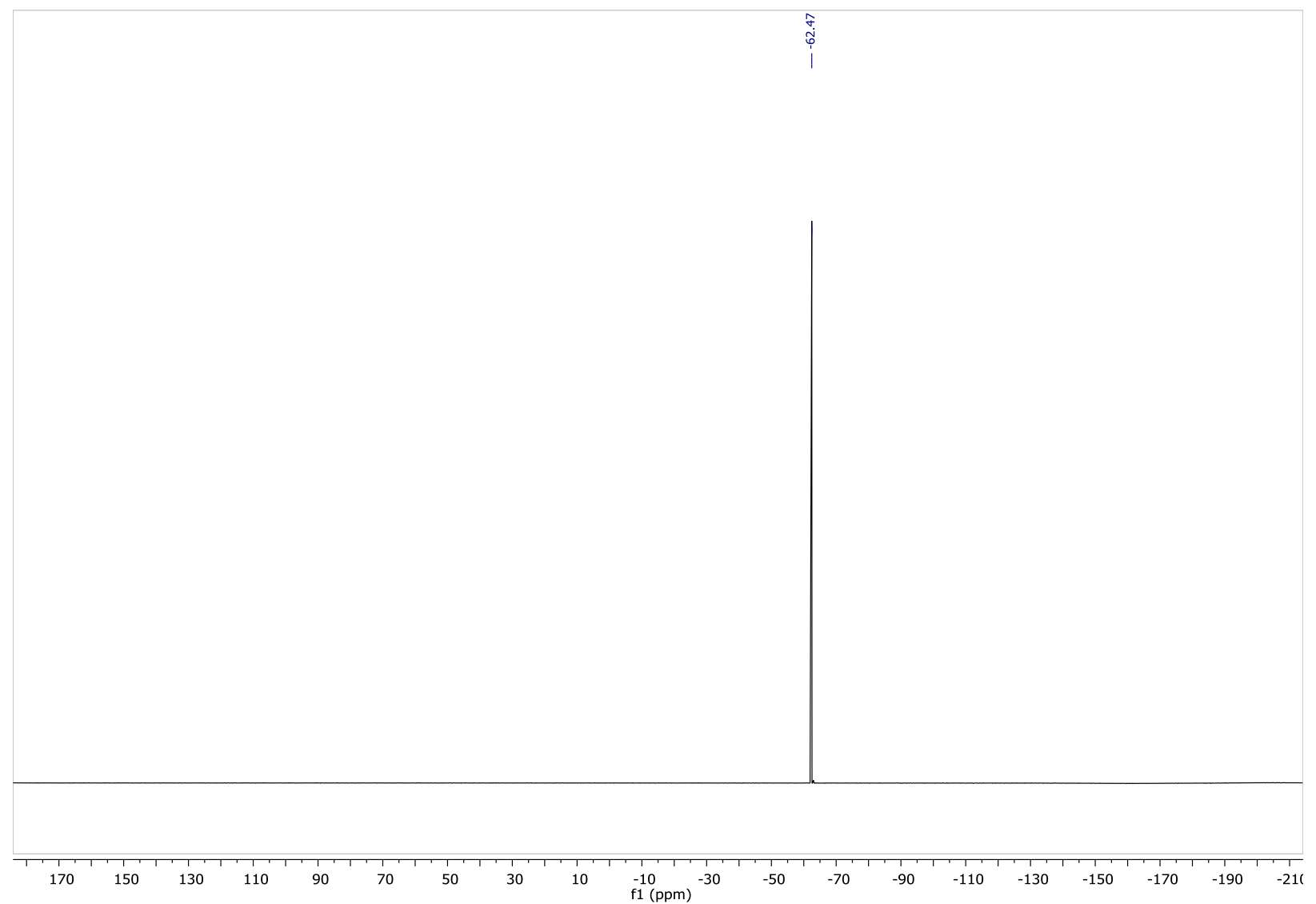



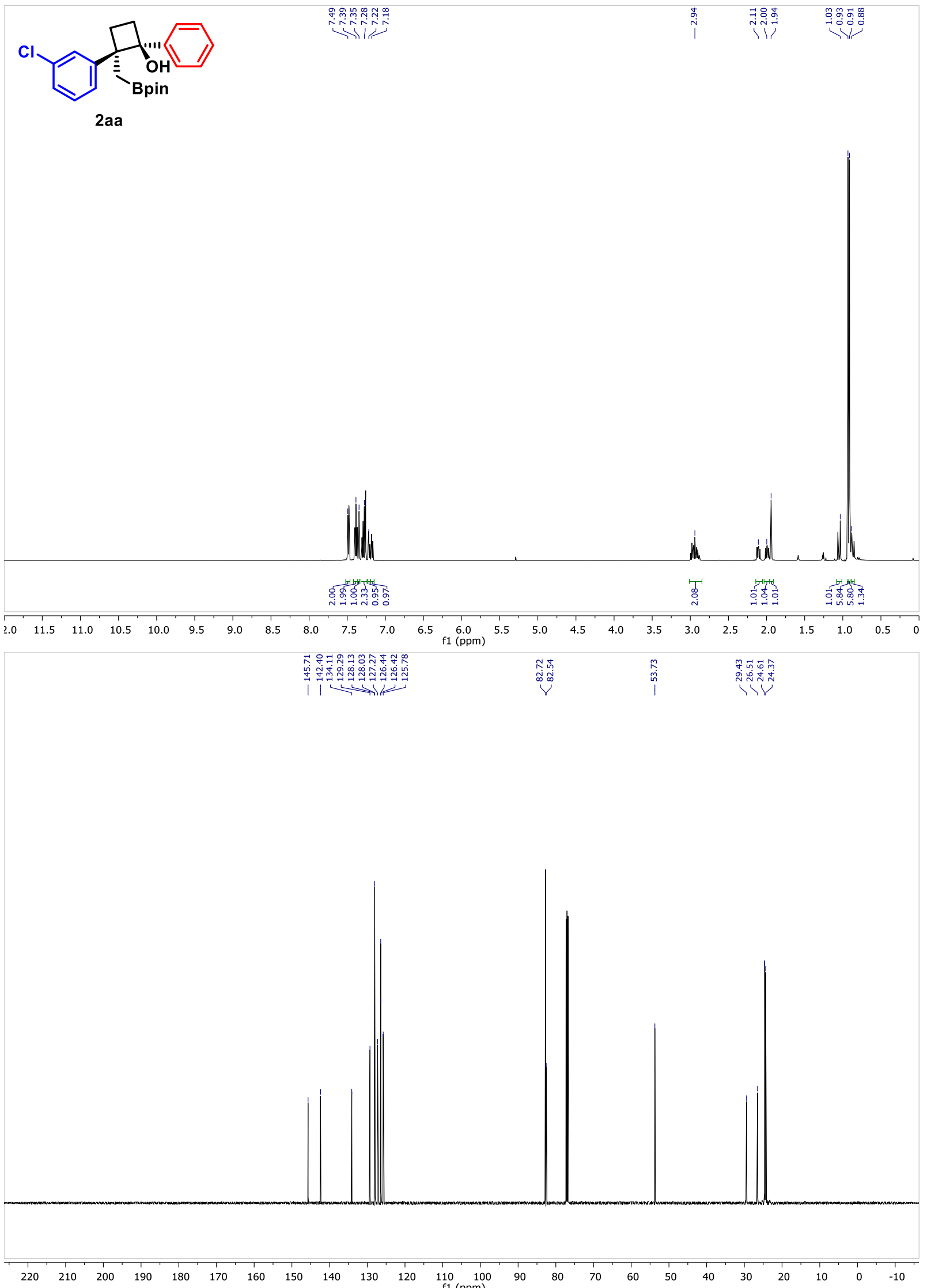


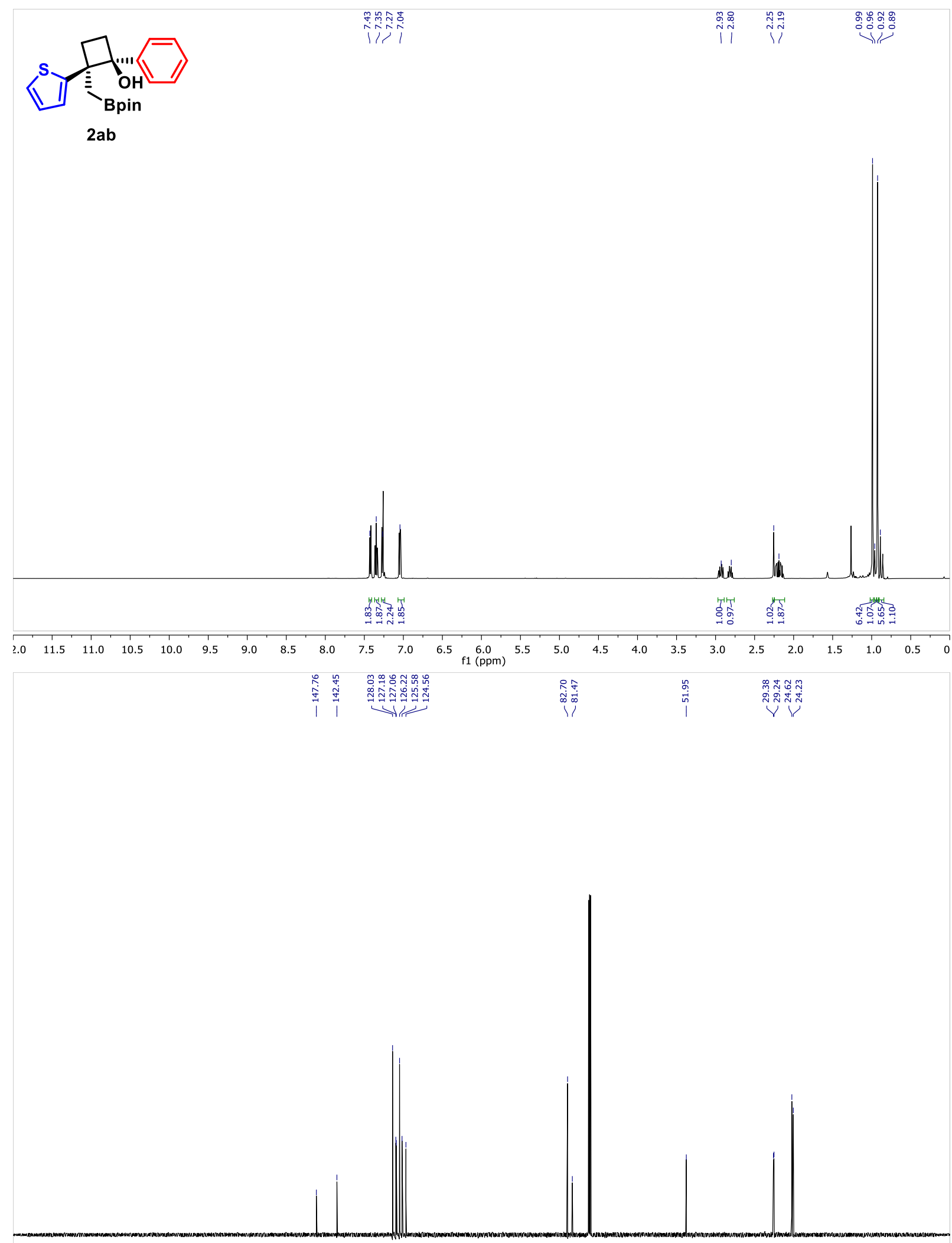



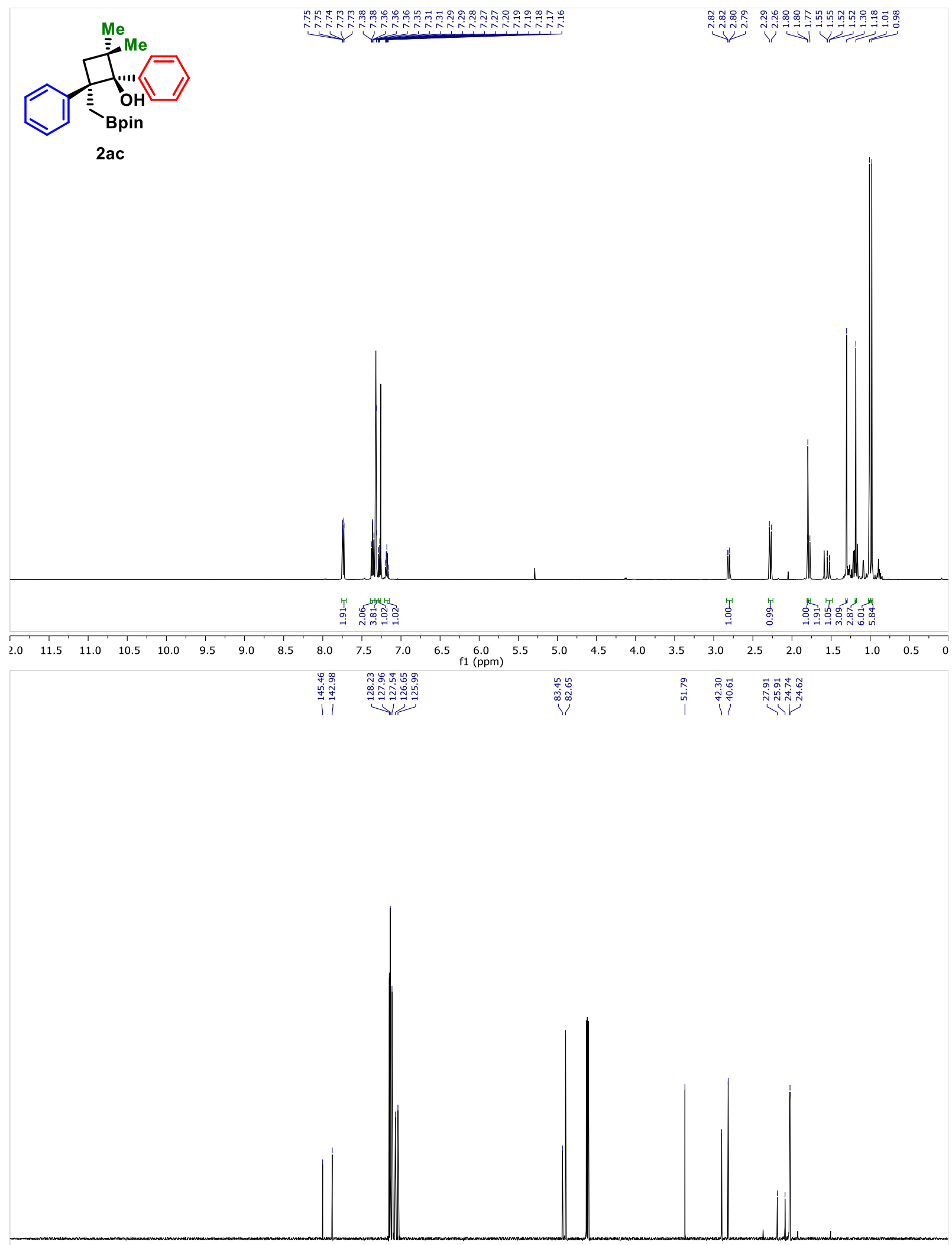

$\begin{array}{lllllllllllllllllllllll}220 & 210 & 200 & 190 & 180 & 170 & 160 & 150 & 140 & 130 & 120 & \begin{array}{c}110 \\ \mathrm{f}(\mathrm{ppm})\end{array} & 90 & 80 & 70 & 60 & 50 & 40 & 30 & 20 & 10 & 0 & -10\end{array}$ 

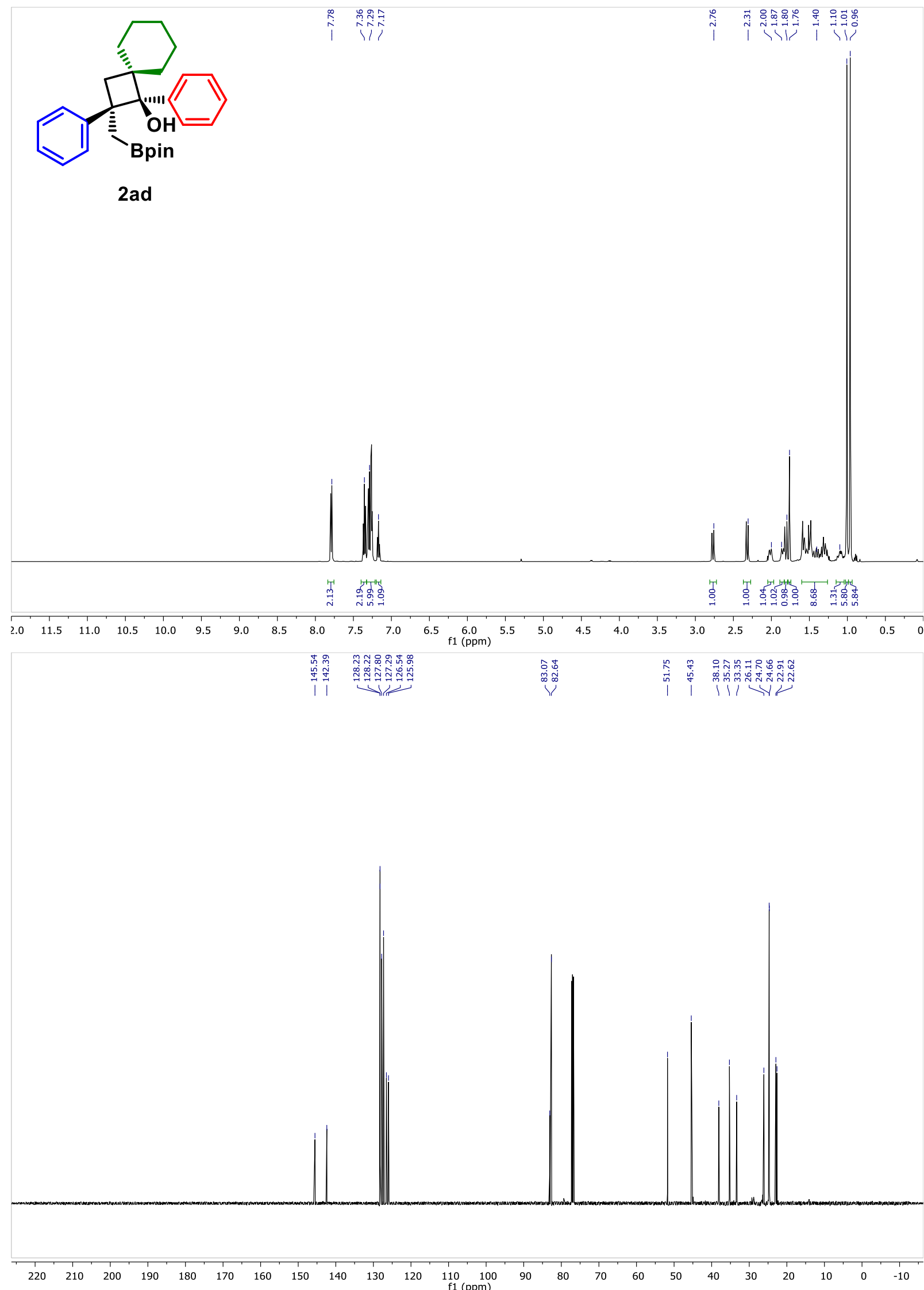


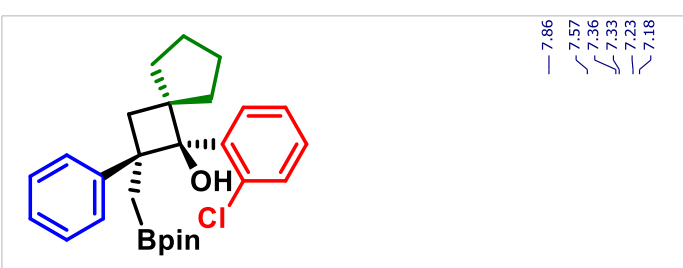

$2 a e$
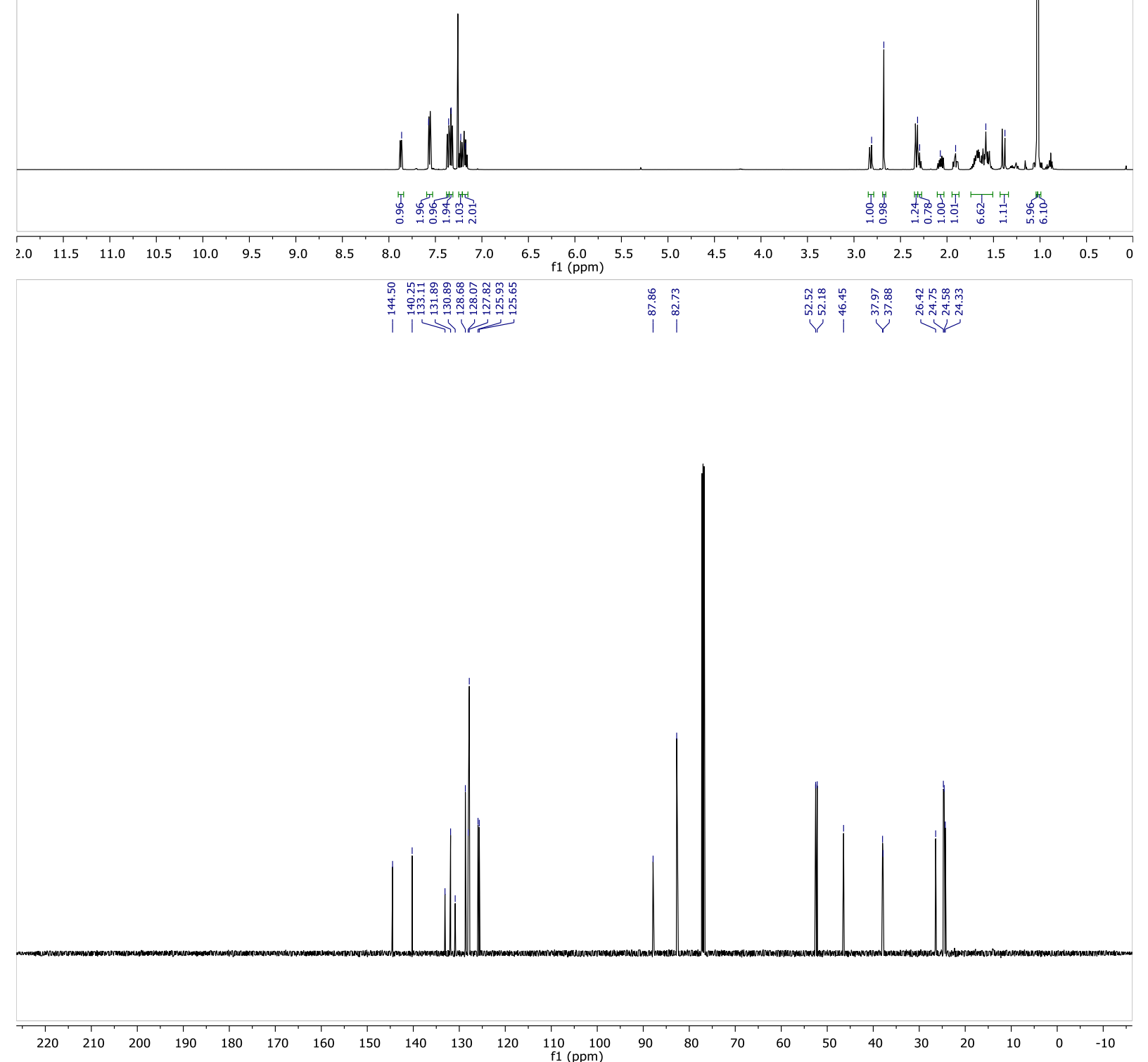

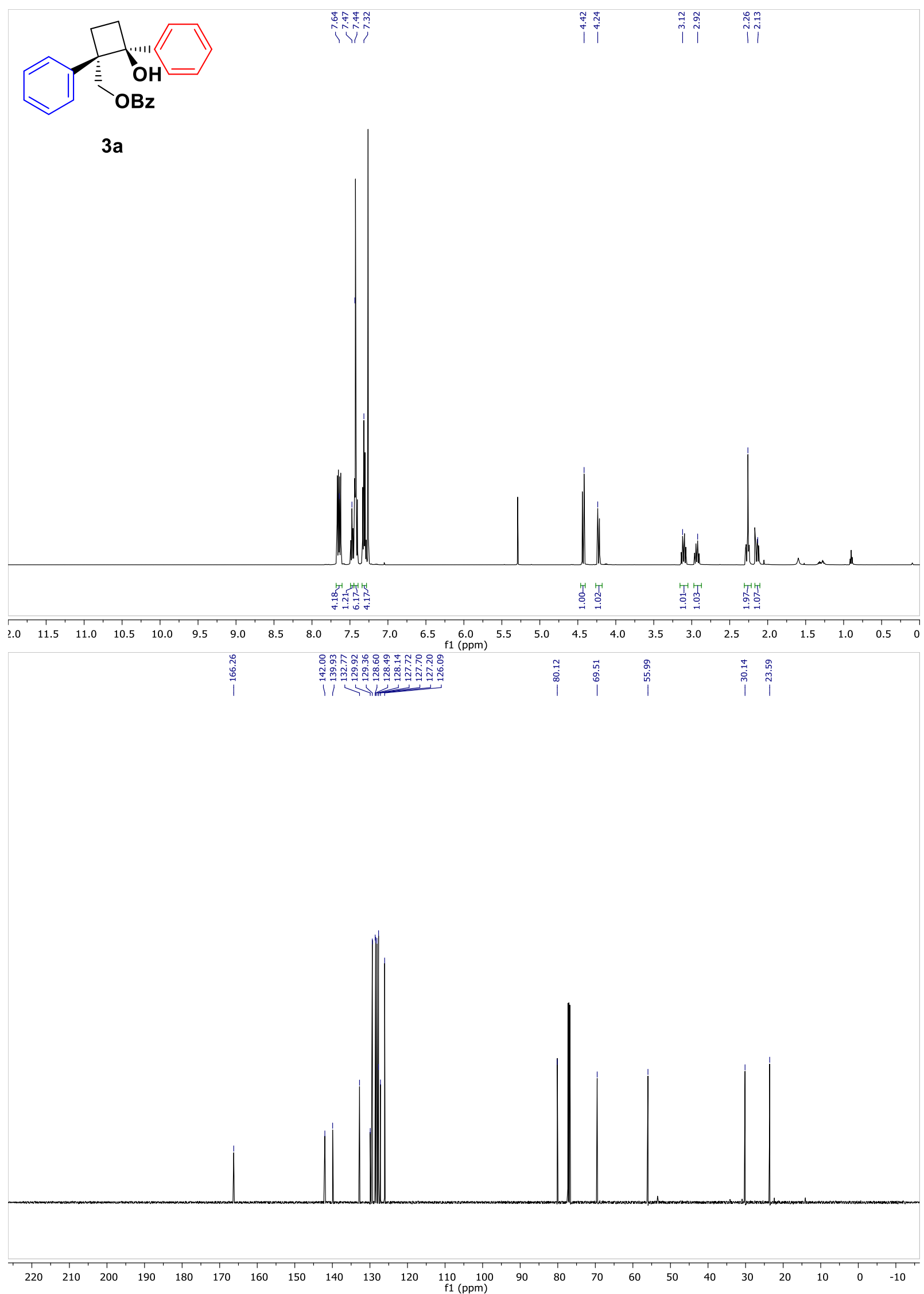

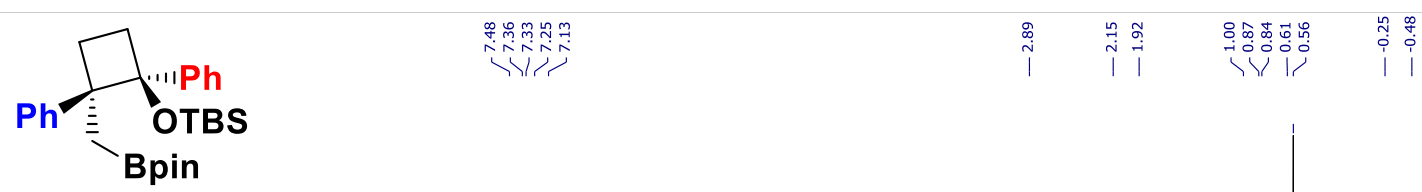

3b
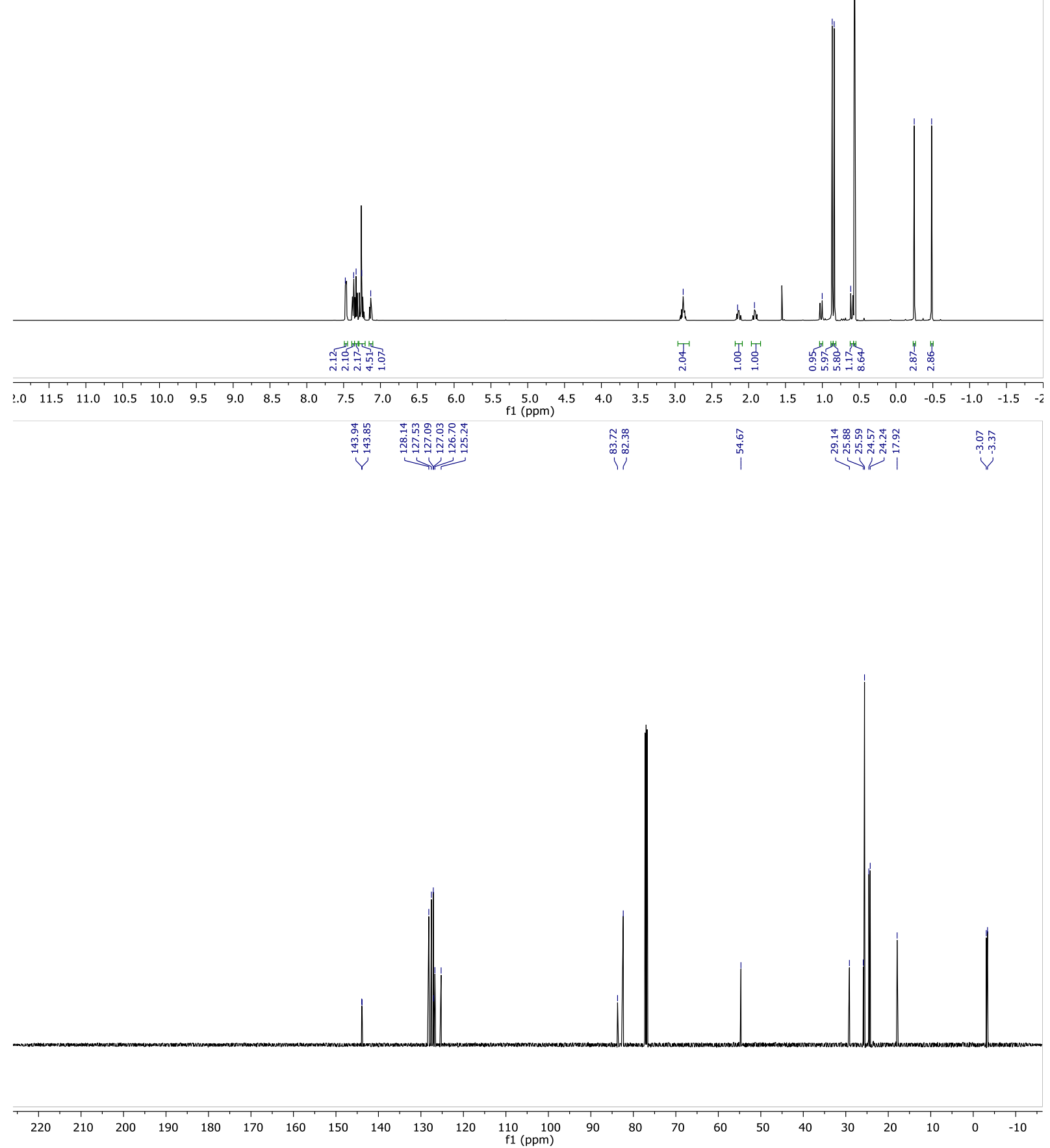

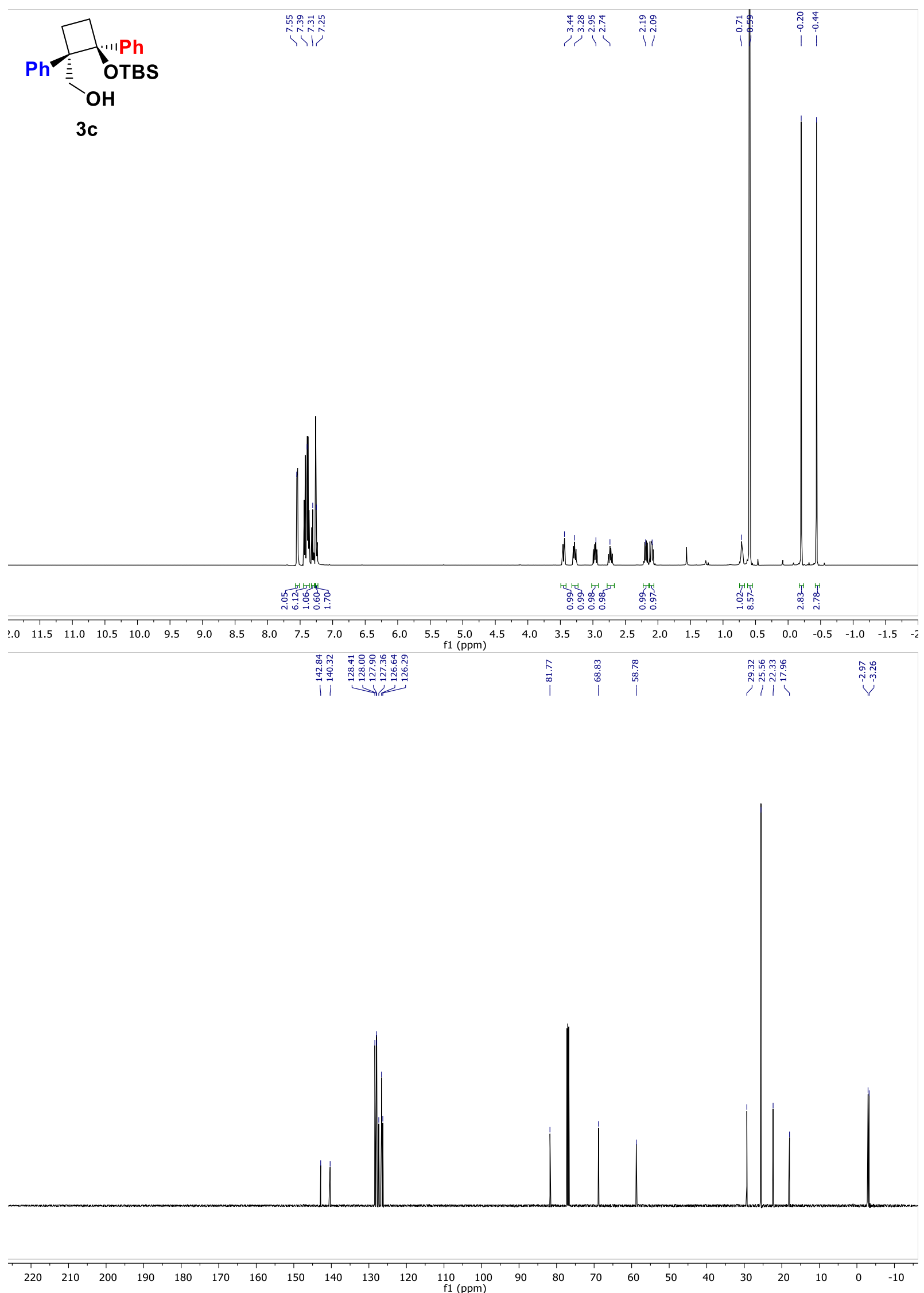

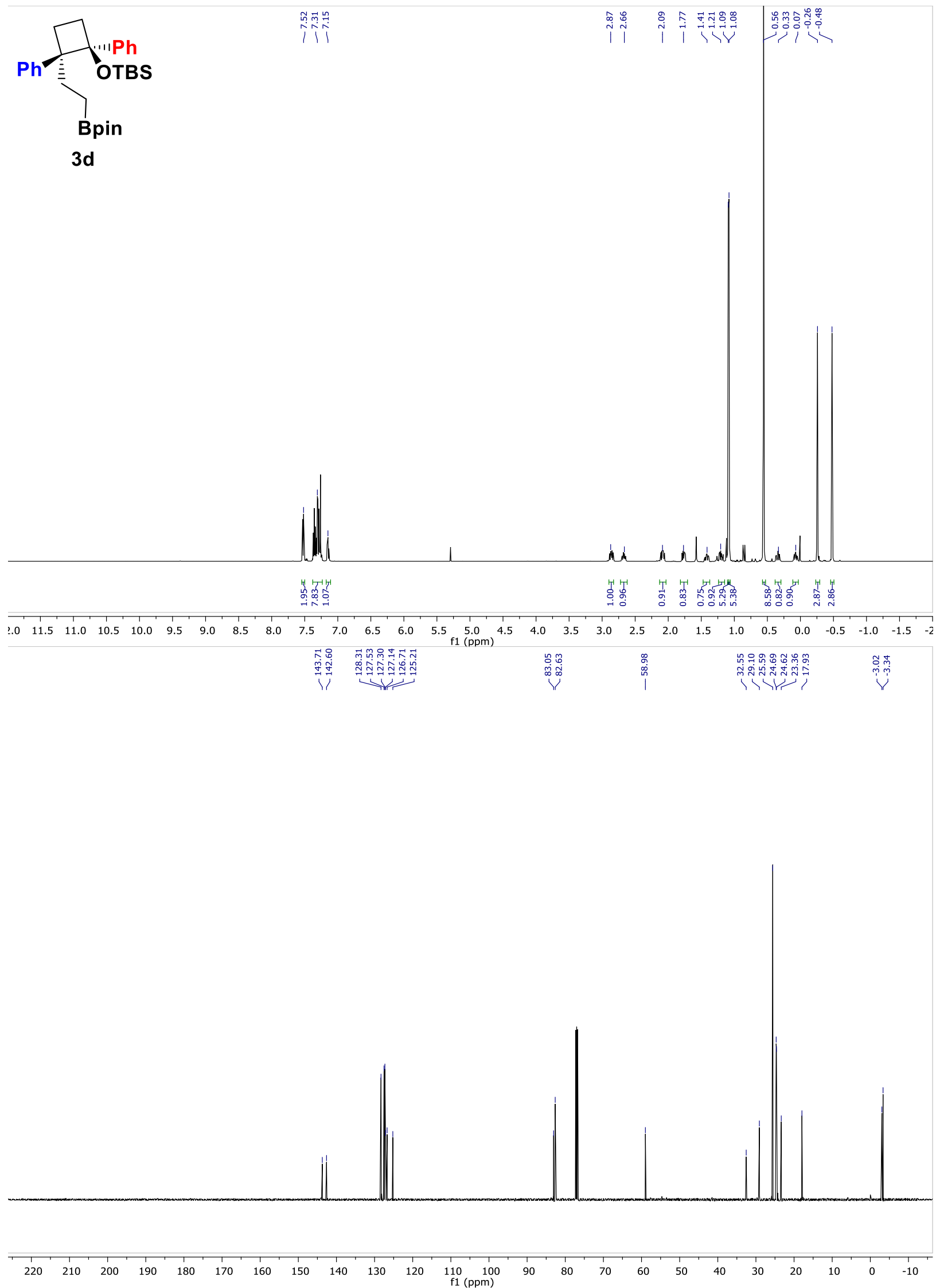

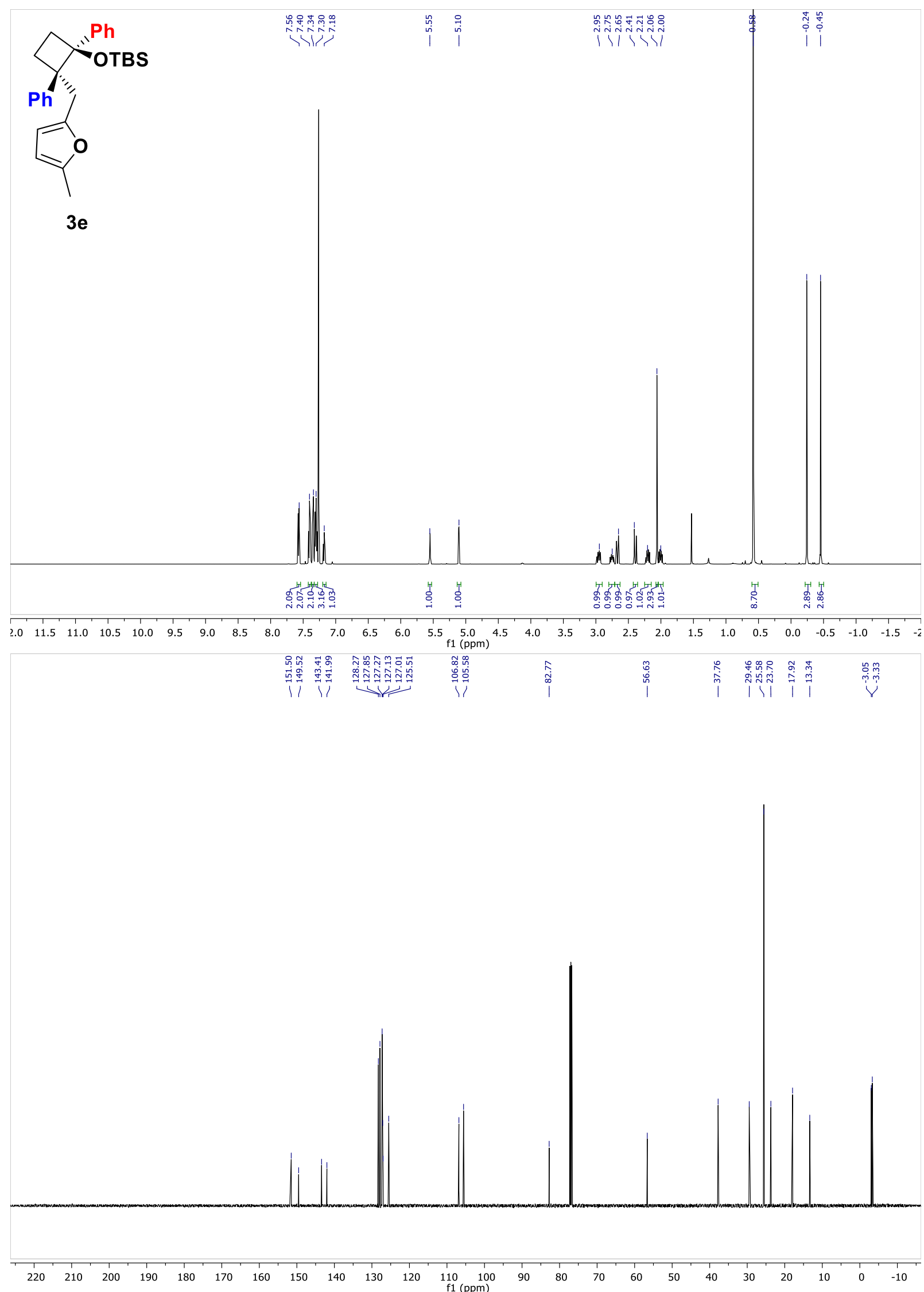

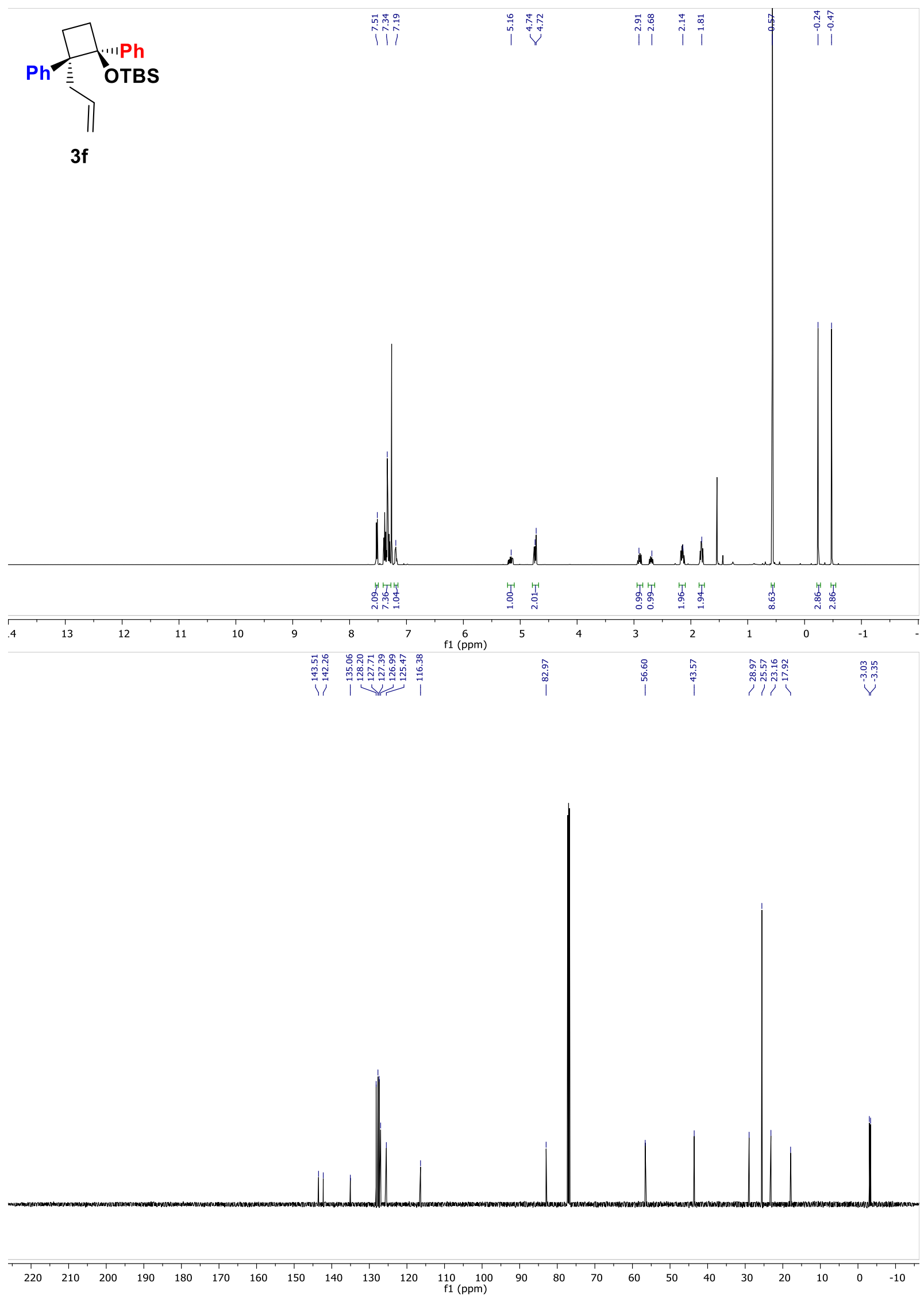